\title{
A Customisable Cell Line Model of Glioblastoma
}

Devlin R. Forsythe

A document submitted to Victoria University of Wellington in partial fulfilment of the requirements for the degree of Master of Biomedical Science

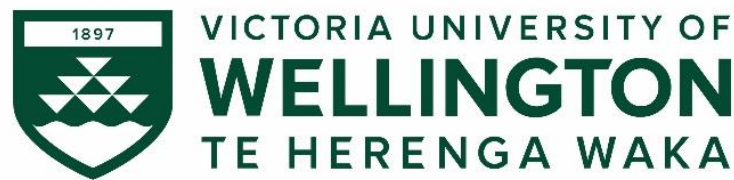

April 2021 


\section{Acknowledgements}

I would firstly like to thank my supervisor, Dr Melanie McConnell. Thank you for giving me the opportunity to start this project. I have learnt so much about the world of research, from a wide range of lab skills to the cutthroat nature of grant applications and funding. I know that your support has helped me develop as researcher, and I look forward to this project continuing into the future.

Thank you to the MMC group. Especially to Anna and Leti for their help in the lab, I have been lucky to have such supportive and knowledgeable people to help me in learning new protocol. Thank you for your help in destressing when things are not going as planned. To Alvey, thank you for teaching us the ways of microbiology and cloning. The weeks spent on plasmid prep went by much better with your guidance.

A big thank you to Sally and Sam at the Malaghan. Without your help on the Influx, my project would not have started off as well as it did. Especially thanks to Sally, who persevered through my initial attempts at transfecting cells. And to Alfonso, thank you for introducing me to the Incucyte which made my data gathering a million times easier than manually assessing the assays (up until it was sent away...).

Thank you to my family. To Mum and Dad, thanks for supporting me through to this stage of my study. The years of support through swimming and study have helped me to produce this and keep my life in order. To Beth, thanks for setting aside the time to help edit this and improve my scientific writing, I really appreciate it (here's to many more scientific chats which the rest of the family cannot decipher). To Aunty Lili, thank you for supporting my goal of becoming a cancer researcher. Without your interest and skills, I doubt I would have achieved what I have today. To Lauren, thank you for putting up with my stress behind closed doors and staying by my side.

Thank you to Bry and Katja who read through the initial drafts of this thesis. Without it, who knows whether this would be legible or not. I also would not have had a lifetime fix of coffee in the space of a year, so thanks for that too. 


\section{Abstract}

Glioblastoma is an extremely malignant brain tumour with one of the lowest survival rates of all cancers. Current treatments do very little to alter this prognosis. Research into new therapies and the biology of glioblastoma has made scarce progress over the past decades. This is partly due to the combination of the tumour's heterogeneity, and the inability of the current animal models to accurately depict this. This project was a pilot study into the development and characterisation of a novel cell line model of glioblastoma, which could be transplanted into immune competent mice, in order to study the disease.

An immortalised C57BL/6 astrocyte cell line, with an EGFP transgene, was used as the base to add glioblastoma specific mutations. To produce a 'classical-like' glioblastoma model, a knockout in Pten was induced, onto which two separate lines the human oncogenes, EGFRVIII and RAS V12, were stably expressed. 'Secondary-like' models were created with a knockout of P53, and the stable transfection of IDH1R132H.

The 'classical-like' cell lines were assessed for how well they mimicked a classical glioblastoma. The Pten knockout cell line showed an increased proliferative and metabolic rate compared with the astrocytes and a significant increase in clonogenicity. The addition of RAS V12 to the cells showed an increased migratory capacity; and the Pten + EGFRVIII cell line had a tendency towards an increased proliferation. The 'secondary-like' cell lines were assessed for their survival-related phenotypes. The P53 cell line showed a decreased proliferative rate, but with an increased metabolic rate and clonogenic ability. The introduction of the IDH1 mutant protein resulted in a decreased rate of G2 arrest in response to ionising radiation.

These cell lines recapitulated what is seen in human glioblastoma tumours and show potential as a transplantation model. Future research will investigate the tumorigenicity of these cell lines. 


\section{Table of Contents}

Acknowledgements ...............................................................................................................ii

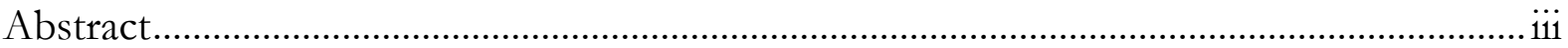

Table of Contents.....................................................................................................................

Table of Figures ................................................................................................................

List of Abbreviations ...........................................................................................................

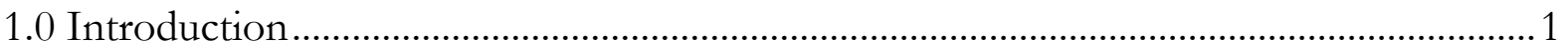

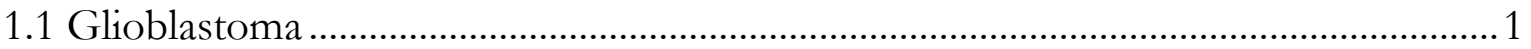

1.1.1 Glial Cells ...........................................................................................................

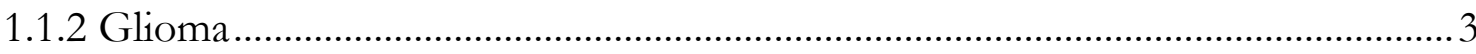

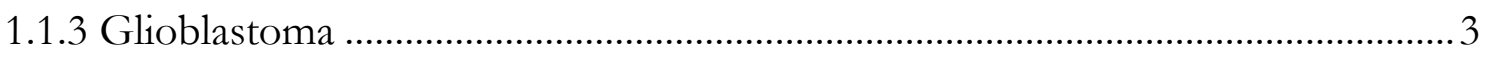

1.2 Heterogeneity and Glioblastoma ..............................................................................

1.2.1 Primary and secondary glioblastoma ................................................................... 5

1.2.2 Subtyping and classification......................................................................... 6

1.2.3 Inter-tumoural heterogeneity and the cell of origin in glioblastoma .................11

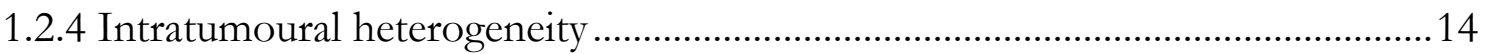

1.3 Animal Models and Glioblastoma.............................................................................. 16

1.3.1 The use of models in glioblastoma ..................................................................16

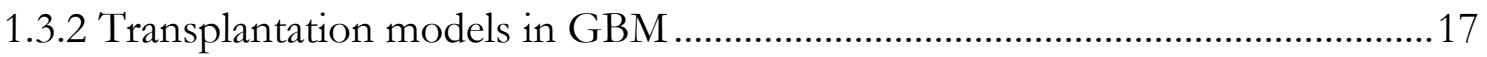

1.3.3 Limitations of the current transplantation models in GBM research................20

1.3.4 Genetically Engineered Mouse Models .............................................................22

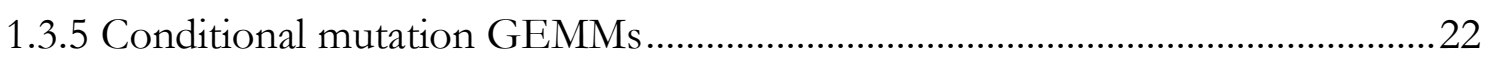

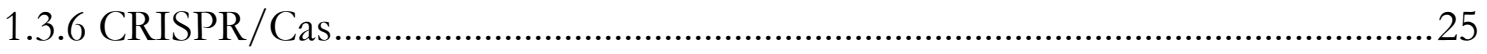




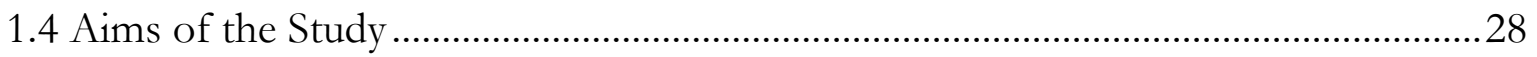

1.4.1 A Customisable Cell Line Model of Glioblastoma .............................................28

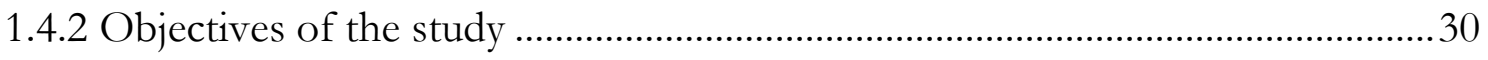

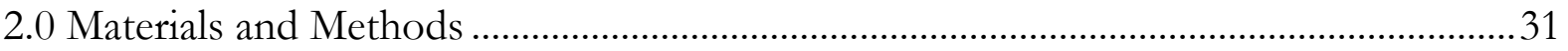

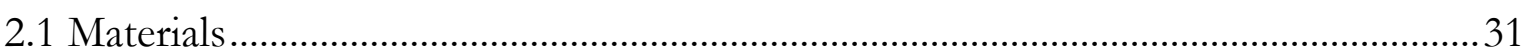

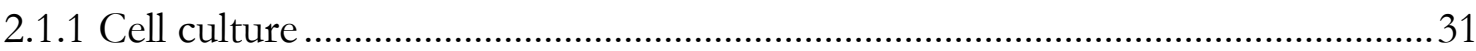

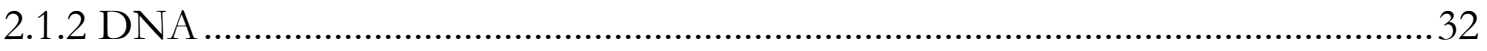

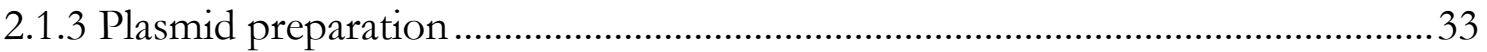

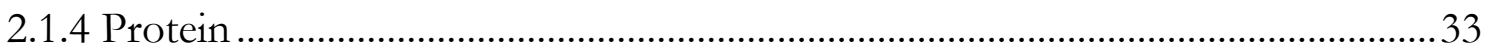

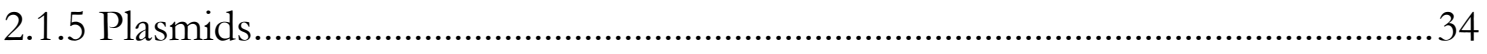

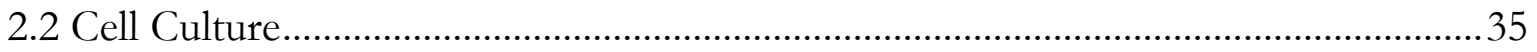

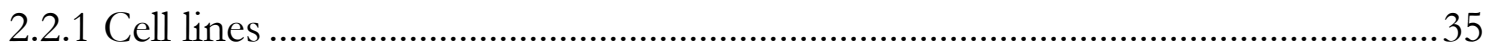

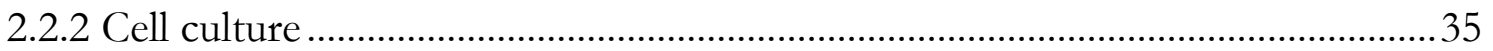

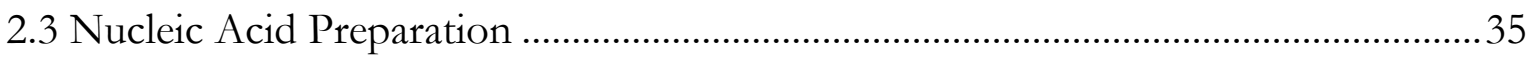

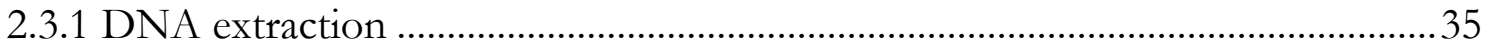

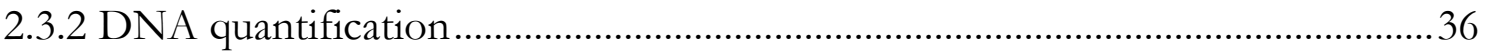

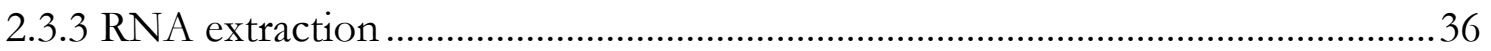

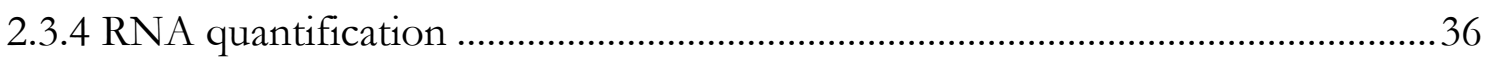

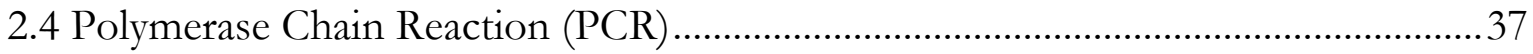

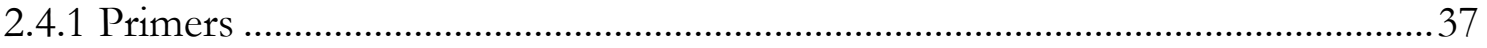

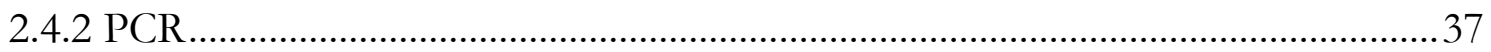

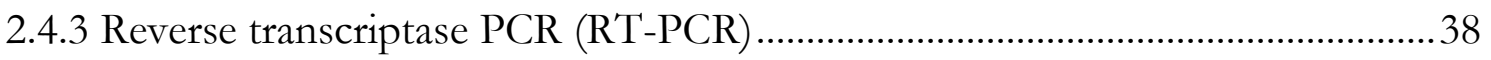

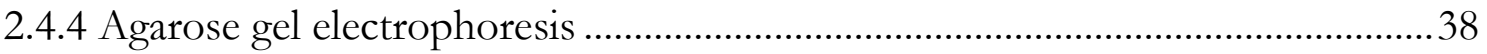

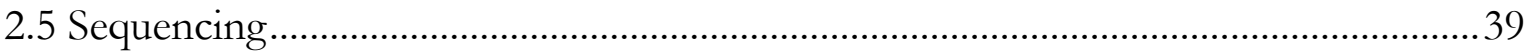


2.5.1 PCR product clean and concentrate

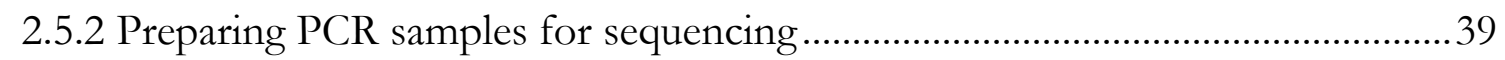

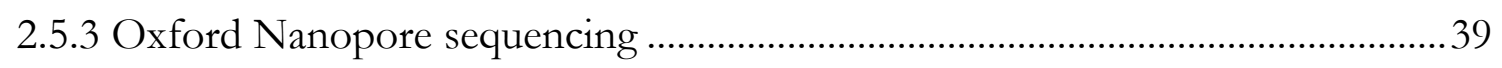

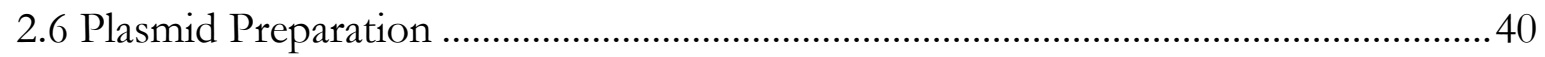

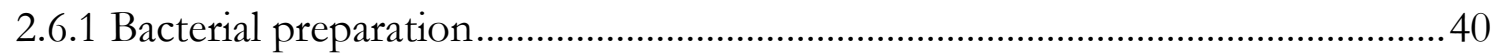

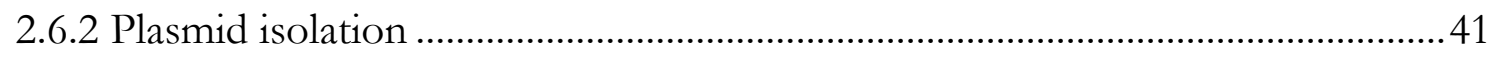

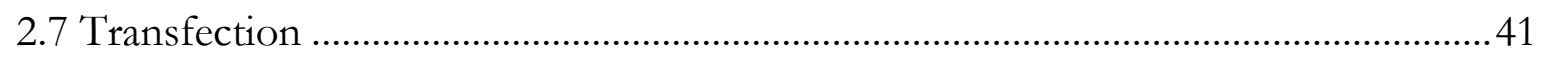

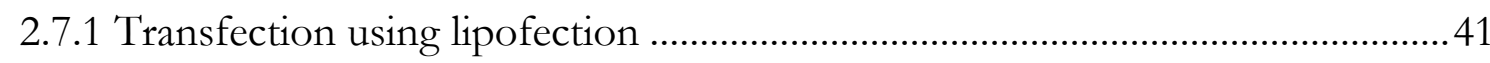

2.7.2 Fluorescence activated cell sorting (FACS) of transfected cells .........................42

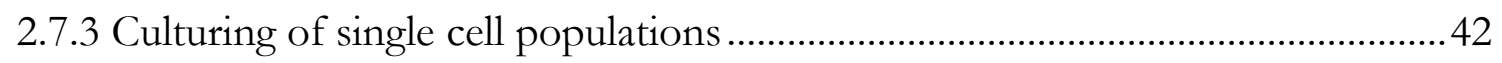

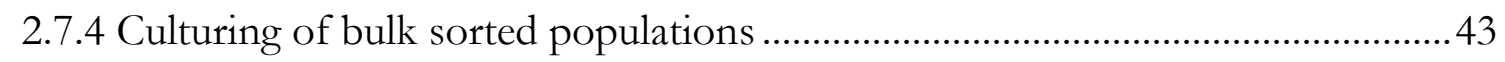

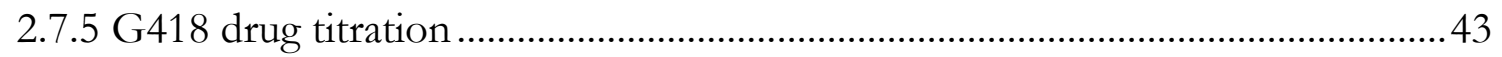

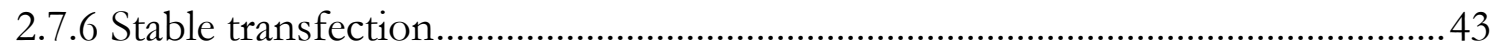

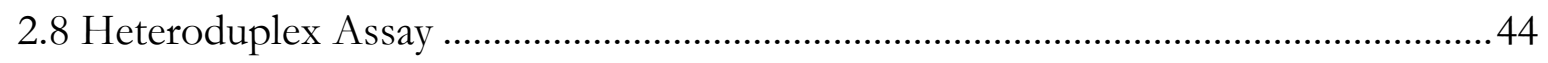

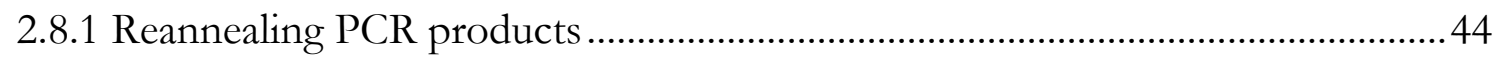

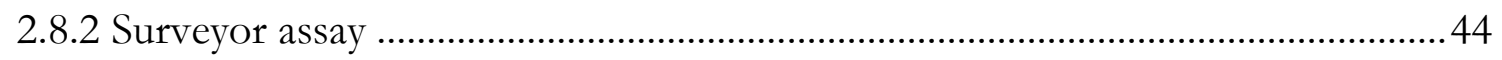

2.8.3 Nondenaturing acrylamide gel electrophoresis .................................................4 44

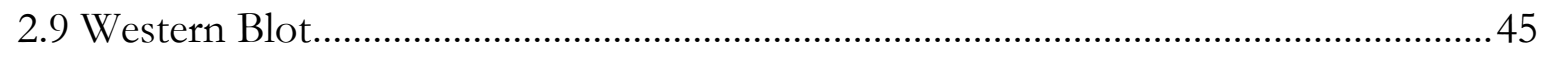

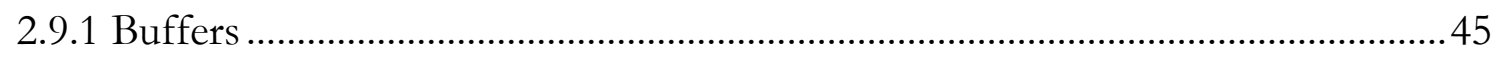

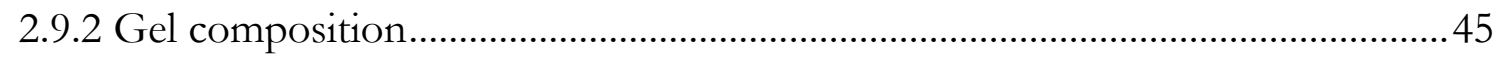

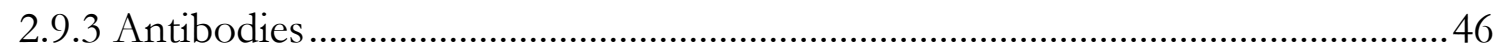

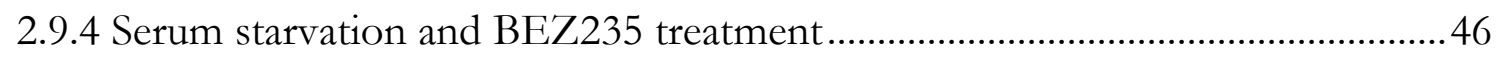

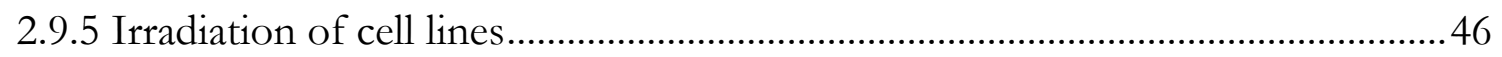

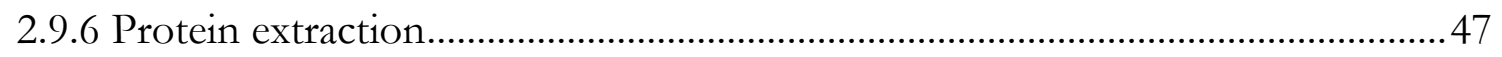

2.9.7 Protein quantification and preparation .......................................................... 47 
2.9.8 SDS-polyacrylamide gel preparation

2.9.9 SDS-polyacrylamide gel electrophoresis (SDS-PAGE) ….................................48

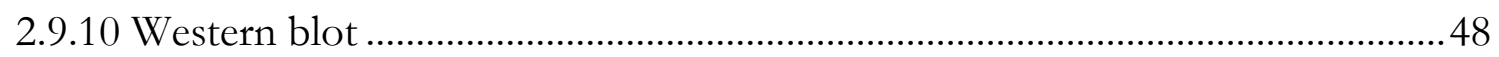

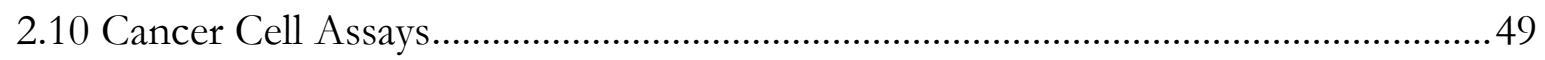

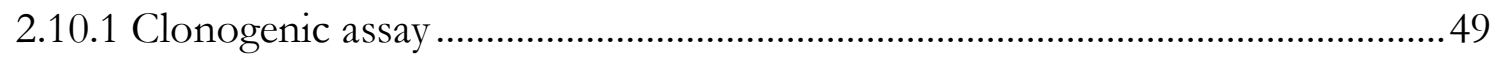

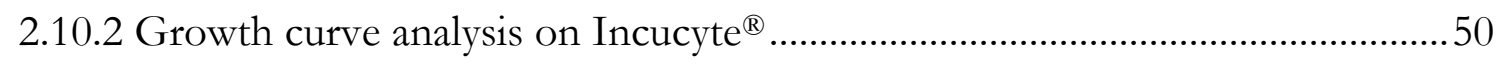

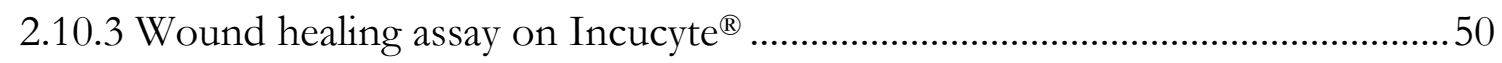

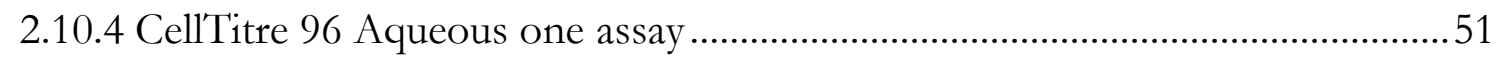

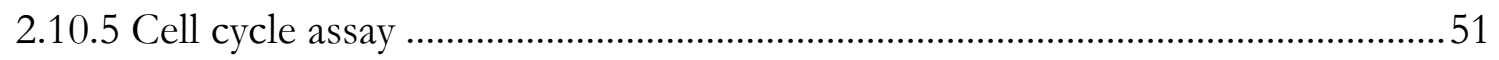

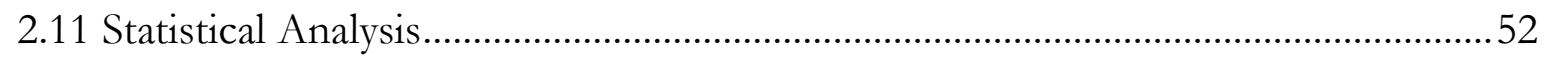

3.0 Development and Screening of CRISPR/Cas9 Induced Knockouts of Pten and P53

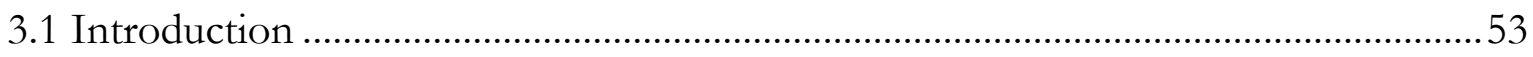

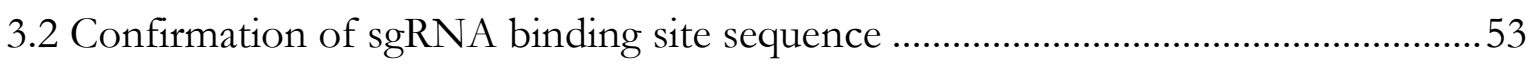

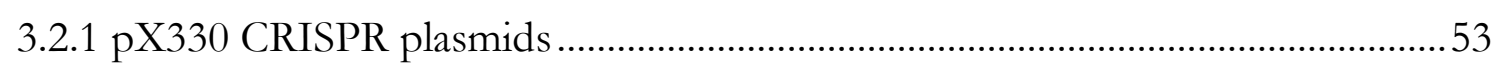

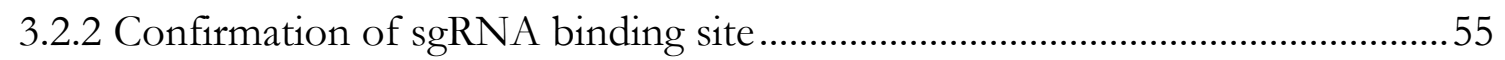

3.3 Development of Pten and P53 knockout mutations .................................................. 57

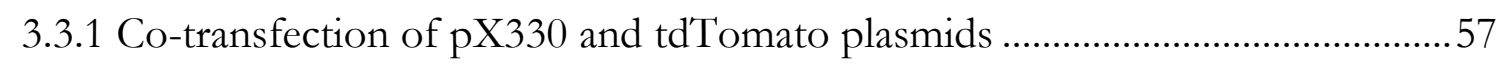

3.3.2 Culturing the bulk population of transfected cells ...........................................60

3.2.3 Culturing the clonal population of transfected cells .........................................60

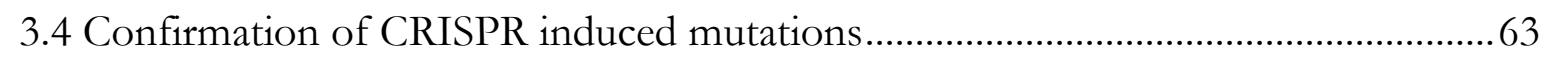

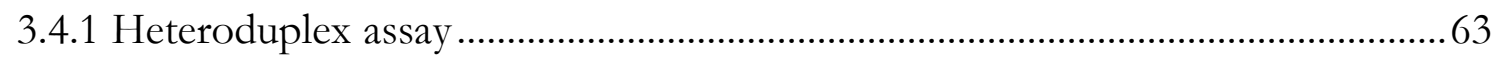

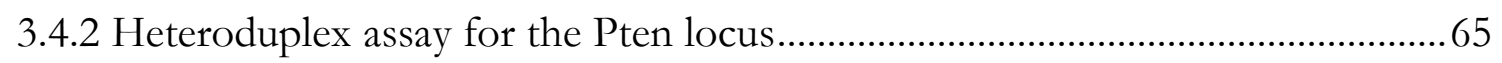

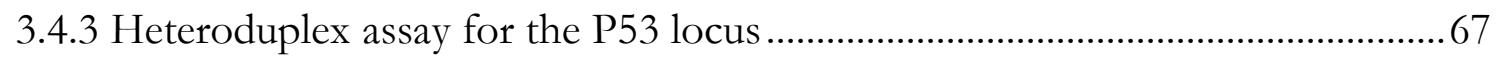

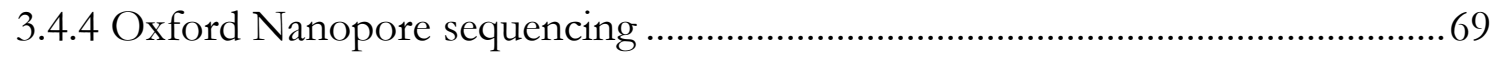


3.4.6 Confirmation of Pten loss by western blot .71

3.4.7 Confirmation of P53 loss by western blot..........................................................

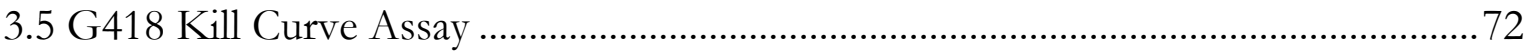

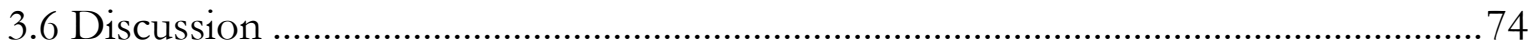

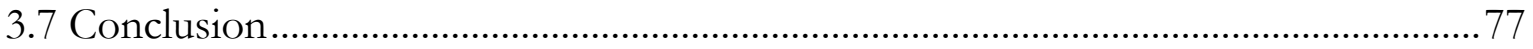

4.0 Development and Characterisation of the 'Classical-Like' GEM-CLEMs ....................78

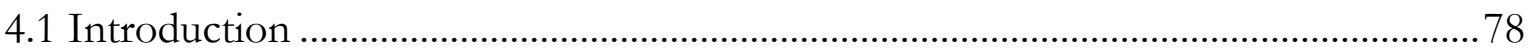

4.2 Addition of EGFRVIII and RAS V12 Oncogenes to C57C3 Pten ...........................78

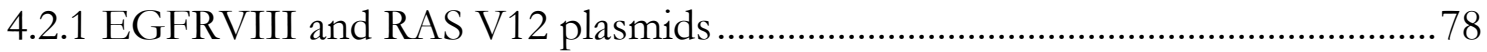

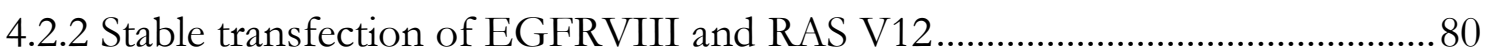

4.2.3 Reverse transcription PCR to confirm oncogene expression ............................. 80

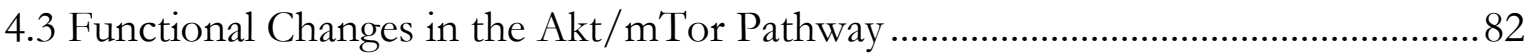

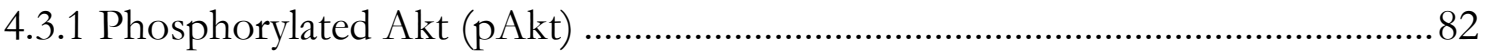

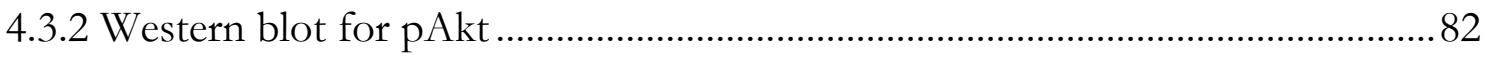

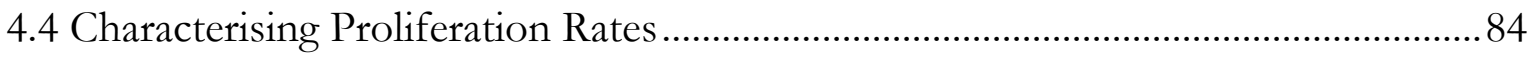

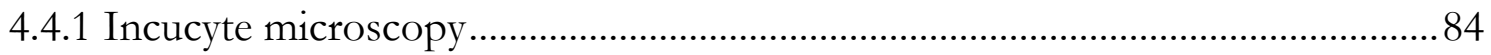

4.4.2 Comparing C57C3 Pten and astrocyte proliferation rate ...................................84

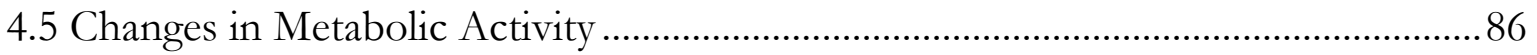

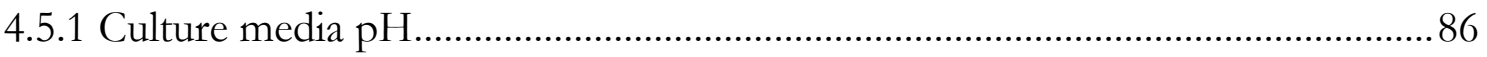

4.5.2 Reductive activity of C57C3 Pten and astrocytes ................................................ 88

4.5.3 Reductive activity of the 'classical-like' GEM-CLeMs .......................................... 90

4.6 Characterising Migration of the Cell Lines ............................................................. 92

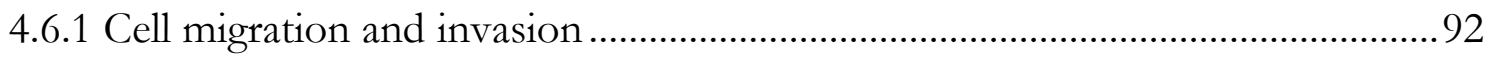

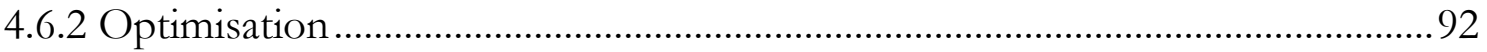

4.6.3 Comparing C57C3 Pten and astrocyte migrative ability …...............................93 


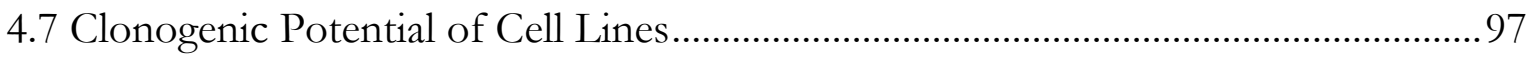

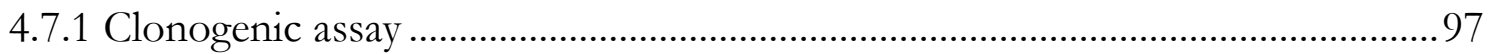

4.7.2 Comparing the C57C3 Pten and astrocyte clonogenic ability ............................98

4.7.3 Comparing the 'classical-like' GEM-CLeMs clonogenic ability .......................100

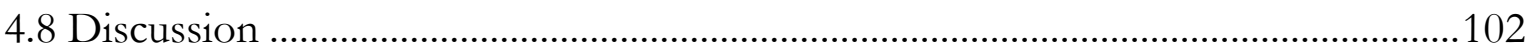

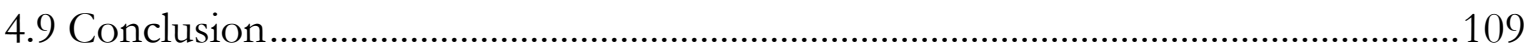

5.0 Development and Characterisation of the 'Secondary-Like' GEM-CLeMs ...............110

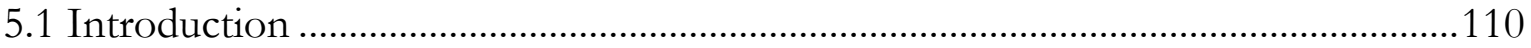

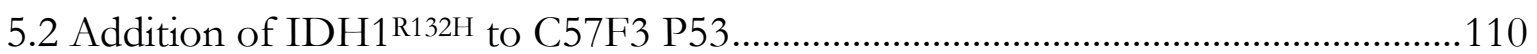

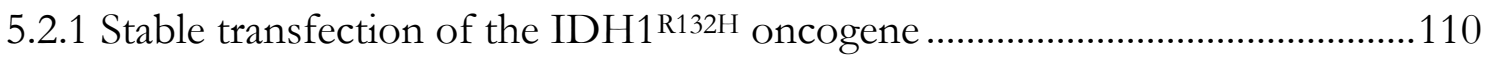

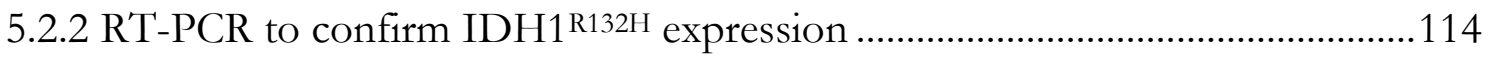

5.3 Functional Changes from P53 Mutation ..................................................................115

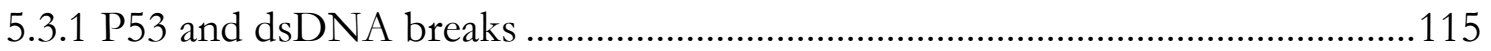

5.3.2 Changes in cell cycle regulation in the C57F3 P53 cells ..................................116

5.3.3 Changes in cell cycle regulation in 'secondary-like' GEM-CLeMs ..................118

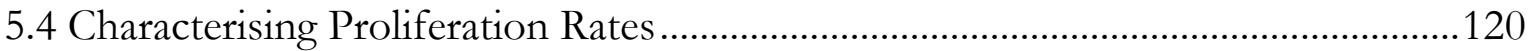

5.4.1 Comparing C57F3 P53 and astrocyte proliferation rate ....................................120

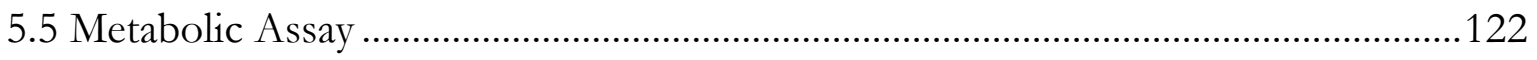

5.51 The ‘secondary-like' GEM-CLeMs culture media pH level.................................122

5.5.2 Reductive activity of C57F3 P53 and astrocytes...............................................124

5.5.3 Reductive activity of 'secondary-like' GEM-CLeMs..........................................126

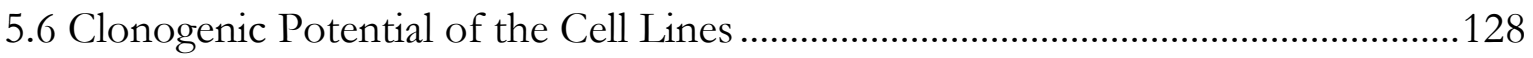

5.6.1 Comparing clonogenic abilities of the C57F3 P53 and astrocyte......................128

5.6.2 Comparing the clonogenic abilities 'secondary-like' GEM-CLeMs ..................130 


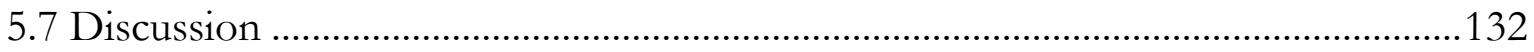

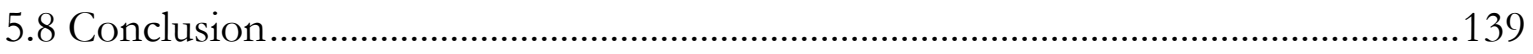

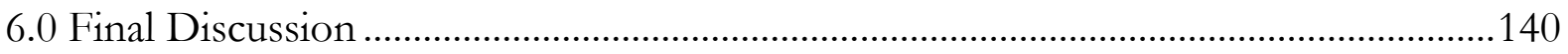

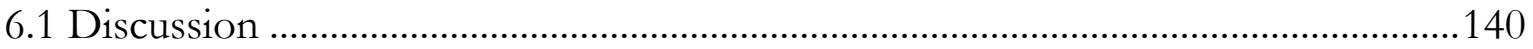

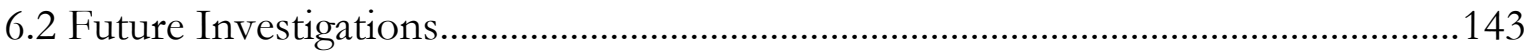

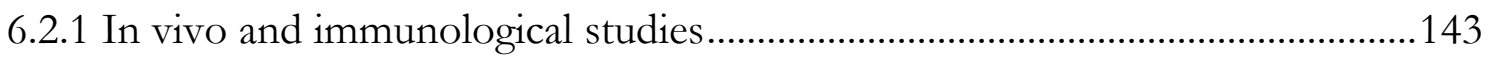

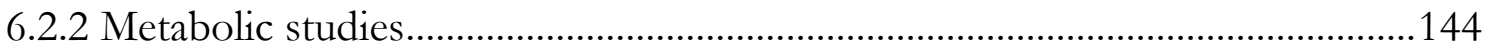

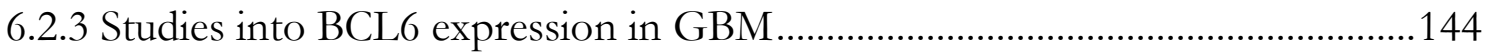

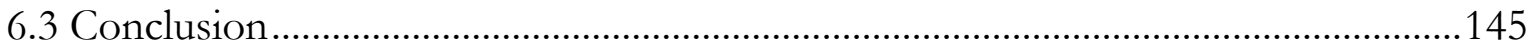

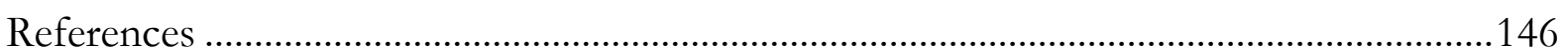

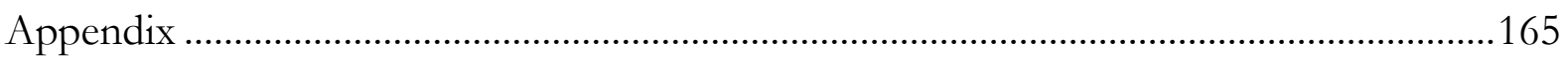

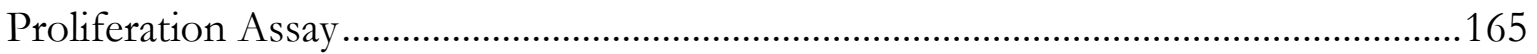

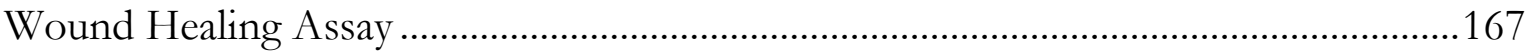

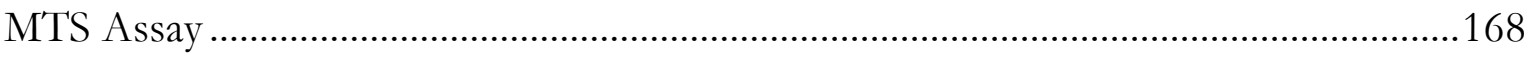

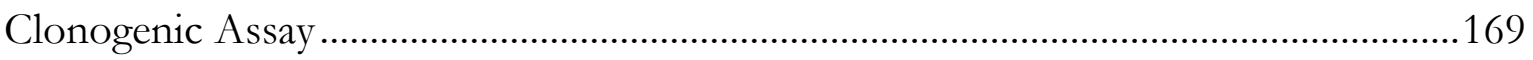




\section{Table of Figures}

Figure 1.1: Progression and differentiation of CNS cell populations. ................................... 2

Figure 1.2: AKT/mTOR signalling pathway................................................................... 7

Figure 1.3: EGFR and MAPK signalling pathway........................................................... 8

Figure 1.4: The interaction between IDH1 and histone demethylation. ............................10

Figure 1.5: Candidates for GBM cell of origin. ................................................................ 14

Figure 1.6: Patient derived xenograft (PDX) transplantation design. .................................19

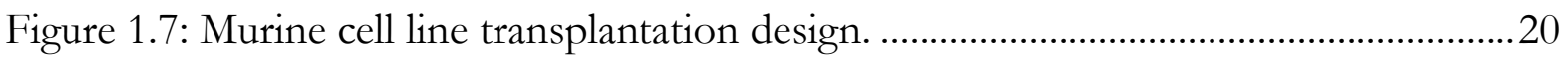

Figure 1.8: Conditional knockout of P53 via Cre/LoxP design. ........................................24

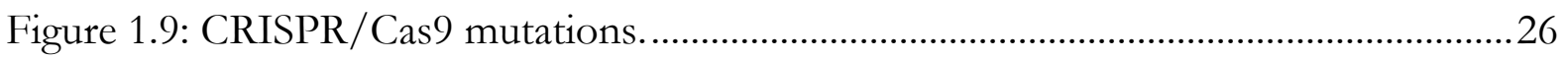

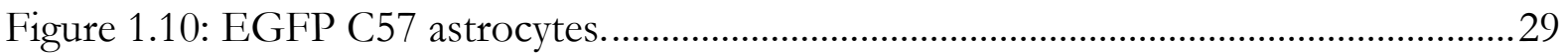

Figure 1.11: Graphical abstract for the project design. ........................................................30

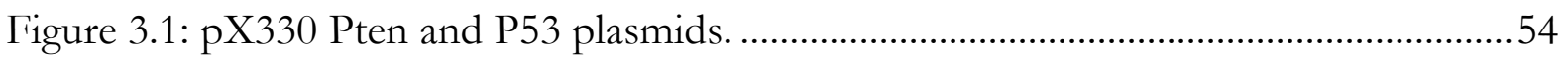

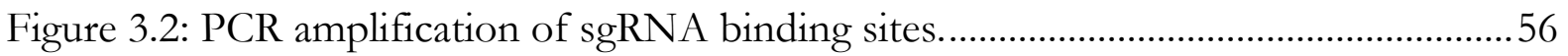

Figure 3.3: Strategy for developing CRISPR knockout clonal populations. .......................57

Figure 3.4: Co-transfection of pX330 plasmids and tdTomato..........................................59

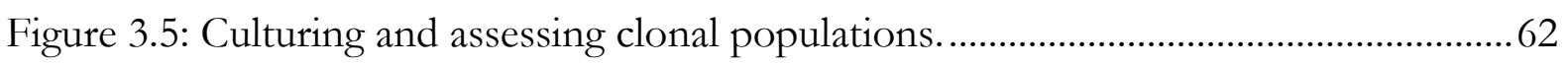

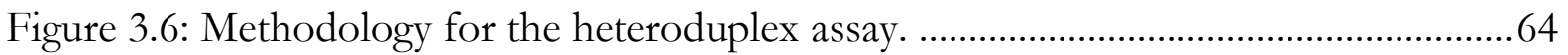

Figure 3.7: Heteroduplex screening for Pten indel mutations. ...........................................66

Figure 3.8: Heteroduplex screening for P53 indel mutations...............................................68

Figure 3.9: Oxford Nanopore MinION sequence confirmation of indels..........................70

Figure 3.10: Western blot for Pten protein abundance....................................................... 71 
Figure 3.11: Western blot for P53 protein abundance.

Figure 3.12: G418 drug kill curve.

Figure 4.1: Oncogene plasmids transfected into the C57C3 Pten cell line .79

Figure 4.2: RT-PCR confirmation of oncogene stable transfections. .81

Figure 4.3: Western blot for pAkt in 'classical-like' cell lines. .83

Figure 4.4: Proliferation rate comparison for astrocytes and C57C3 Pten cells. .85

Figure 4.5: pH Changes in the 'classical-like' culture media $\mathrm{pH}$. .88

Figure 4.6: Comparison of MTS assay in astrocytes and C57C3 Pten cells. .89

Figure 4.7: Comparison of MTS assay in 'classical-like' GEM-CLeMs. 91

Figure 4.8: Example of the migration observed in the wound healing assay. .93

Figure 4.9: Wound healing assay for astrocytes and C57C3 Pten cells. 94

Figure 4.10: Wound healing assay for C57C3 Pten and Pten + RAS cells. .96

Figure 4.11: Example of the clonogenic assay analysis using Image J.... .97

Figure 4.12: Clonogenic assay results for astrocytes and C57C3 Pten cells. .99

Figure 4.13: Clonogenic assay results for 'classical-like' GEM-CLeMs. 101

Figure 4.14: Normalised data comparing the astrocytes and C57C3 Pten assays. 104

Figure 4.15: TCGA (2013) data on PTEN and EGFR mutations frequency in GBM. ..107 Figure 4.16: TCGA (2013) overall survival data for patients with EGFR and PTEN mutations. 108

Figure 5.1: IDH1R132H mutant plasmid for stable transfection.

Figure 5.2: G418 selection for IDH1 ${ }^{\mathrm{R} 132 \mathrm{H}}$ plasmid transfection. 
Figure 5.4: Cell cycle assay after radiation for astrocytes and C57F3 P53 cells.

Figure 5.5: Cell cycle assay after radiation for 'secondary-like' GEM-CLeMs..................118

Figure 5.6: Proliferation assay for astrocytes and C57F3 P53 cells. ..................................121

Figure 5.7: pH Changes in the 'secondary-like' culture media $\mathrm{pH}$...................................122

Figure 5.8: MTS Assay for astrocytes and C57F3 P53 cells. ............................................125

Figure 5.9: MTS Assay for ‘secondary-like’ GEM-CLeMs...............................................127

Figure 5.10: Clonogenic assay for astrocytes and C57F3 P53 cells.................................129

Figure 5.11: Clonogenic assay for 'secondary-like' GEM-CLeMs.....................................131

Figure 5.12: Normalised data comparing the astrocyte and C57F3 P53 assays...............133

Figure 5.13: TCGA (2013) data on P53 and IDH1 mutations frequency in GBM.........137

Figure 5.14: TCGA (2013) overall survival data for patients with P53 and IDH1 mutations. 138

Table 2.1: Primers used in polymerase chain reaction experiments throughout the thesis.

Table 2.2: Protocol for thermocycler used for polymerase chain reaction throughout the thesis. .38

Table 2.3: Antibodies used throughout the thesis. .46 


\section{List of Abbreviations}

CNS

OPC

BBB

NSC

SGZ

SVZ

LGG

HGG

GBM

TMZ

TCGA

$\alpha \mathrm{KG}$

D2HG

sgRNA

PDX

G-CIMP

GEMM
Central Nervous System

Oligodendrocyte Precursor Cell

Blood Brain Barrier

Neural Stem Cell

Sub-granular Zone

Subventricular Zone

Low-grade Glioma

High-grade Glioma

Glioblastoma

Temozolomide

The Cancer Genome Atlas

$\alpha$ Ketoglutarate

D-2-Hydroxyglutarate

Short Guide RNA

Patient Derived Xenograft

Glioma-CpG Island Methylator Phenotype

Genetically Modified Mouse Model 
PAM

NHEJ

HDR

Indel

GEM-CLeM

FBS

DMEM

PBS

APS

G418

$\mathrm{NBF}$
Protospacer Adjacent Motif

Non-homologous End Joining

Homology Directed Repair

Insertion/Deletion

Genetically Engineered Mouse Cell Line Model

Foetal Bovine Serum

Dulbecco’s Modified Eagle Medium

Phosphate Buffered Saline

Ammonium Persulfate

Gentamicin Sulfate

Neutral Buffered Formalin 


\subsection{Introduction}

\subsection{Glioblastoma}

\subsubsection{Glial Cells}

The brain is composed of two major cell types, neurons and glial cells. Neurons form the functional unit of the central nervous system (CNS), whereas glial cells form the supporting network. There are three major glial cells in the brain: astrocytes, oligodendrocytes, and microglia ${ }^{1,2}$. These cells regulate the structure of the neurons, and protect the brain from damage ${ }^{1}$.

Astrocytes occupy approximately half of the cell mass of the brain and have the most varied roles in the CNS. Most of these involve regulating the homeostasis of the brain 2,3, an example of this is seen through their control of extracellular glutamate levels ${ }^{4}$. While glutamate is an important neurotransmitter in the CNS, an increase in concentration in the synaptic cleft is neurotoxic and leads to cell death. Astrocytes have the critical role of taking up the excess levels of glutamate and regulating its release to the excitatory neuron ${ }^{4}$. As well as regulation of CNS homeostasis, astrocytes are essential in establishing the brain's functional structure. Their extending arms contribute to the formation of synaptic junctions in CNS neurons, and to the formation of the blood-brain barrier ${ }^{2}$. The complete role of astrocytes in the brain is not fully understood, yet it is clear that astrocytes are necessary for maintaining the appropriate function of the brain itself 3 .

Oligodendrocytes aid the CNS in a structural capacity. Derived from oligodendrocyte precursor cells (OPCs), a form of glial precursor, these cells produce the myelin sheath which surrounds the axon of neurons, both protecting the cells and increasing the speed of electrical transmission by up to $150 \mathrm{~ms}^{-1}{ }^{5}$. This process of myelination begins at two years of age and continues until the mid-twenties, and disruptions can lead to major nervous system damage and degradation ${ }^{2}$. Unlike astrocytes, 
which are limited to the brain and CNS, oligodendrocytes have a peripheral nervous system (PNS) counterpart in the Schwann cell ${ }^{6}$.

Microglia function entirely differently than the other glial cells. Being derived from myeloid precursors, microglia are the immediate innate immune cell of the brain (Fig 1.1). The CNS is largely immune restricted, and access is tightly regulated via the blood-brain barrier (BBB) and the choroid plexus. As a result, the microglia have an important role in the immediate control of brain pathology, acting as a macrophage ${ }^{7}$.

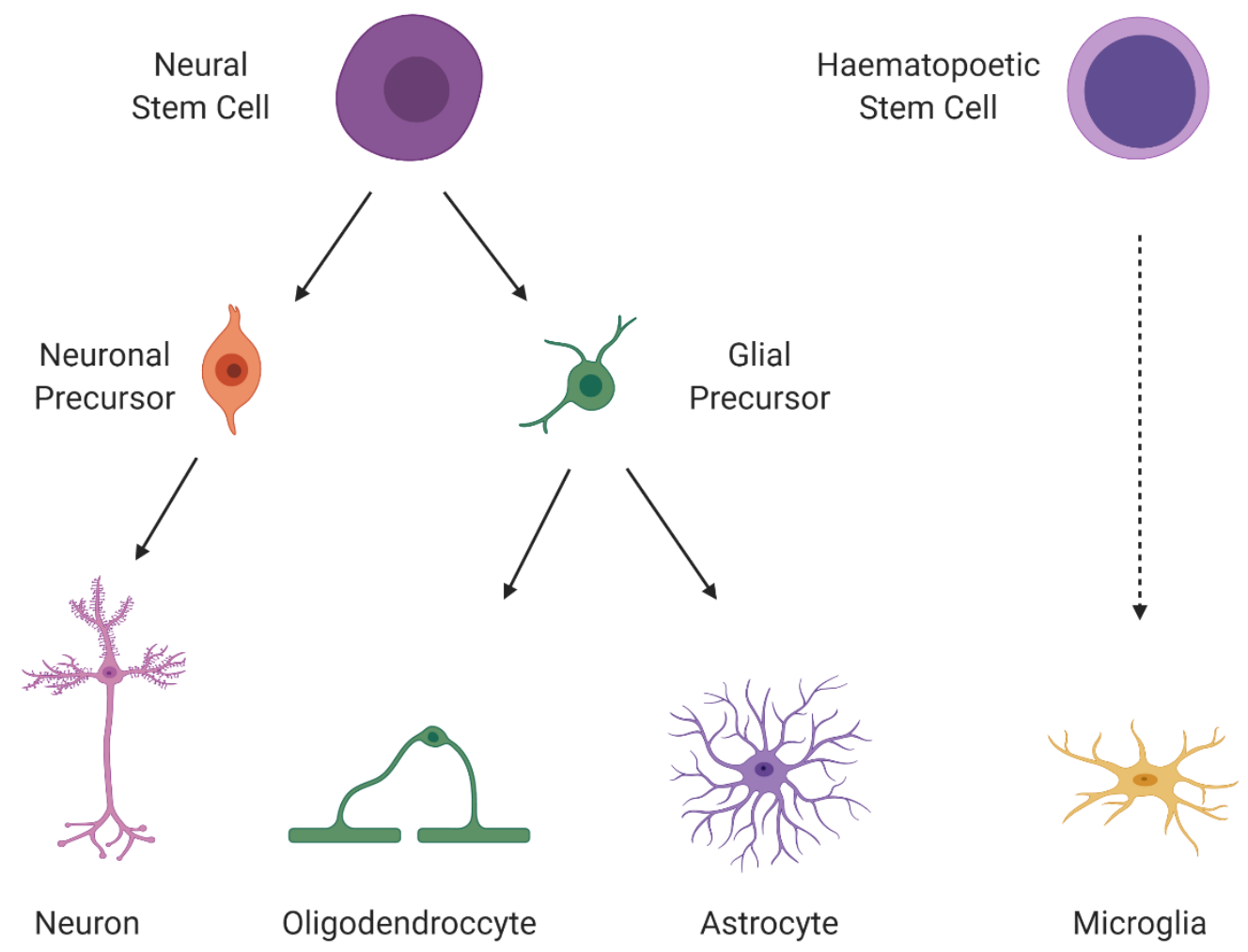

Figure 1.1: Progression and differentiation of CNS cell populations. Created with BioRender.

The remainder of the CNS is comprised of the precursor and stem cells. These cells occupy very specific locations in the brain, and once differentiation occurs migration follows. For example, neural stem cells (NSCs) are only found in two locations: the sub- 
granular zone (SGZ) of the dentate gyrus and the subventricular zone (SVZ) of the lateral ventricles. These two sections of the brain are known as neurogenic niches as the specific cellular interactions and cell types present are responsible for adult neurogenesis, in particular for formation of glial cell precursors and neurons ${ }^{8}$.

\subsubsection{Glioma}

Tumours arising from glial cells are known as glioma. Like the cells which they develop from, glioma is a diverse category of tumour ${ }^{9}$. Low-grade glioma (LGG), grades II and III, account for $20 \%$ of all CNS tumours. The most common LGG are oligodendroglioma and diffuse astrocytoma ${ }^{10}$. Both tumours are largely categorised by the presence or absence of isocitrate dehydrogenase 1 (IDH1) mutations, seen in approximately $80 \%$ of LGG. Because of their relatively slow and uniform growth, these tumours have a better five-year survival rate than their high-grade counterparts ${ }^{11}$. Patients who receive a $90 \%$ resection of the tumour show a 97\% five-year survival rate, compared with less than $5 \%$ survival at five years for high grade gliomas (HGG) ${ }^{12}$. These HGGs are a very heterogeneous category of CNS tumour, and are largely characterised by their rapid development and treatment resistance. The most common and malignant of these tumours is glioblastoma (GBM) ${ }^{13}$.

\subsubsection{Glioblastoma}

GBM is the most frequent CNS tumour, accounting for approximately $15 \%$. Additionally, GBM is the most malignant of the tumours to affect the brain; the median life expectancy after treatment is only 14 -months ${ }^{11,13}$. The cause of this poor prognosis is complex, and largely due to the diversity and heterogeneity of GBM. The most defining physical characteristic of GBM is the unique histology. As GBM is such a rapidly growing tumour, there is a need for the central mass to develop its own vasculature to sustain the level of proliferation. However, these tumours develop so rapidly that angiogenesis is not conducted appropriately ${ }^{14}$. As a result, the central mass of GBM tends to die. Histologically this is seen as pseudopalisading necrosis, where the centre of the tumour dies via necrosis 
and this is surrounded by a layer of cells in a 'pseudopalisade' 13,15,16. The surrounding tumour tissue is extremely diffuse and projects into the healthy parenchyma. Most commonly, GBM is located in the lateral side of the brain's ventricular region ${ }^{17}$.

There are typically three treatments given to patients depending on the progress of the tumour; surgery, chemotherapy, and radiation therapy ${ }^{18}$. While not all patients undergo surgery, as this depends on the age at diagnosis or the progression of the tumour, removal of cancerous tissue is commonly the first option in treating GBM. This is because surgical removal of the tumour, either biopsy or resection, is the only method of treatment which reduces the cellular composition of the cancer. However, because of the diffuse borders and the regular $2 \mathrm{~cm}$ safety margin for the surgery, not all of the tumour can be removed. This leads to the tumour recurring ${ }^{19}$.

The frontline chemotherapeutic agent is temozolomide (TMZ). This drug is the only chemotherapy available which actively passes the BBB and has the effect of killing GBM tissue. It is an alkylating agent which disrupts DNA synthesis, resulting in cell death ${ }^{20}$. When combined with a course of radiation therapy - consisting of $60 \mathrm{~Gy}$ over six-weeks targeted on the tumour mass - there is a survival increase. The percentage of people with progression-free survival following a course of radiation therapy is $1.5 \%$ at 24 months $(0.1$ 3.0, 95\% CI), with the concomitant course of TMZ this is increased to $10.7 \%(7.0-14.3$, $95 \%$ CI ${ }^{18}$. However, while this is promising data, this does little to aid the $<5 \%$ five-year survival rate for glioblastoma ${ }^{11}$. This poor prognosis has many roots; but, a major contributor is the presence of the MGMT gene, and the hyper-methylated pattern seen in $50 \%$ of GBM ${ }^{20}$. MGMT is a DNA repair enzyme that specifically repairs altered guanidine residues. This is the type of damage that TMZ treatment introduces. In tumours where the promotor for MGMT is methylated and suppressed, TMZ is much more effective. However, when the gene is active, TMZ induced DNA damage is negated, and cell death is minimal ${ }^{21}$. 


\subsection{Heterogeneity and Glioblastoma}

\subsubsection{Primary and secondary glioblastoma}

GBM is defined as a primary or secondary tumour by its progression and the stages prior to diagnosis ${ }^{13}$. If the tumour arises de novo and is immediately termed a stage IV cancer, it is known as a primary GBM. These tumours tend to be more proliferative and invasive 22,23; because of this, primary GBM has a poorer prognosis than secondary tumours 11,13,24. Secondary GBM are distinct as they progress from an LGG. Because of this, these tumours tend to have a slower progression to the lethal stage ${ }^{25}$. As well as having an altered prognosis, secondary tumours tend to maintain the morphology of the tissue they are derived from, leading to a more differentiated histology. This means that they have more homology with the tissue from which they are derived than primary GBM 19,24.

Secondary GBM have the highest survival rates. These tumours are detected earlier in the disease progression than primary tumours, and more is known about their pathology. Along with LGG, secondary GBM are divided into groups depending on the IDH1 status, with mutants possessing the greatest rates of 5-year survival because of the slower growth of tumours with this mutation, which will be discussed further (1.2.2). Yet mutations to this gene only occur in $6 \%$ of all GBM, whereas primary tumours account for approximately $90 \% 19$.

Typically, primary tumours arise much later in age compared with secondary (median diagnosis of 60 years and 45 years, respectively), yet when the tumours develop it is at a much faster rate ${ }^{13}$. Despite these relatively large differences between the two categories and the slight survival benefits seen with secondary tumours, both forms of GBM have a dismal prognosis. Because of this prognosis, a molecular lens is required when classifying the tumours clinically. 


\subsubsection{Subtyping and classification}

Genomic analysis of tumours through The Cancer Genome Atlas (TCGA) project began in 2008 by sequencing GBM 26. From this data, subtypes of GBM have been identified based on the driver mutations present. These subtypes were proposed by Verhaak et al. (2010) 22 and over time have been revised and refined using all the genomic, transcriptomic and DNA methylation data available for GBM ${ }^{27}$. Three molecular subtypes of GBM have been defined: classical, mesenchymal, and proneural. The most aggressive forms of GBM are usually classified as either the classical or mesenchymal subtypes; these have the poorest prognosis. A loss of the PTEN gene is a frequent mutation seen in these two subtypes 22,28,29. PTEN is an inhibitor of the AKT/mTORC1 pathway, through which it functions to regulate the cellular stress response, resulting in a carefully-regulated proliferative rate (Fig 1.2) ${ }^{30}$. Mutations to PTEN lead to the loss of a crucial regulator of the mitotic pathway and as a result there is an increase in the tumours proliferation ${ }^{30,31}$. 


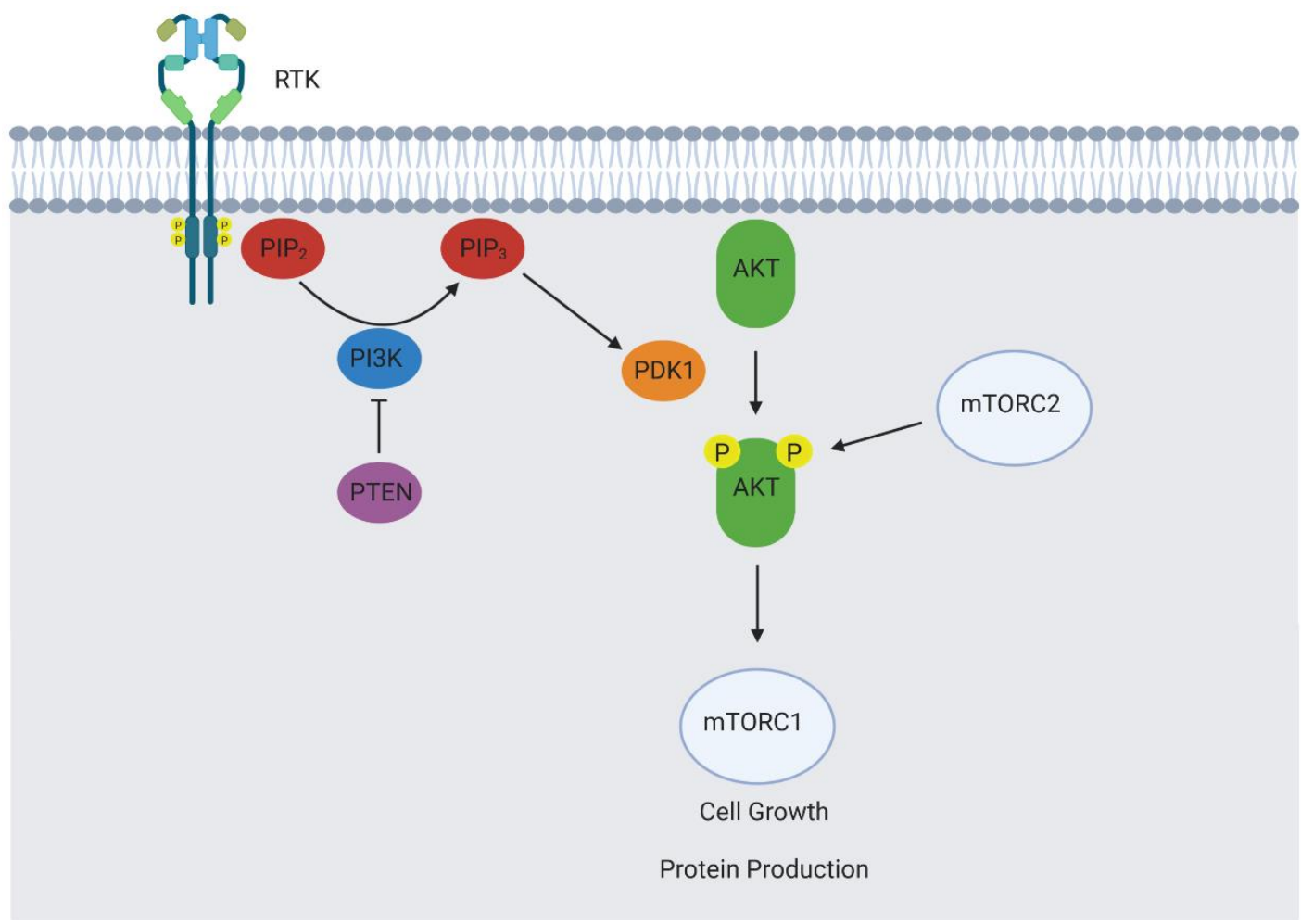

Figure 1.2: AKT/mTOR signalling pathway. Phosphorylation of receptor tyrosine kinases (RTK) shown in yellow leads to recruitment of $\mathrm{PIP}_{2}$. PI3K converts $\mathrm{PIP}_{2}$ to $\mathrm{PIP}_{3}$ which leads to dual phosphorylation of AKT (yellow p) by PDK1 and mTORC2. This results in mTORC1 activation and initiates cell growth and protein production pathways. PTEN is an inhibitor of PI3K. Created with BioRender.

The Classical subtype is characterised by mutations seen in the EGFR locus, either through a focal amplification of the entire chromosome seven or a mutation to the protein itself ${ }^{22}$. The most common point mutation, EGFRvIII, is a gain of function mutation which allows the receptor to be constitutively active and not require epidermal growth factor (EGF) as a stimulus ${ }^{32}$. EGFR is a receptor tyrosine kinase, and as such is involved in many signalling pathways, including AKT-mTOR. However, the greatest effect this mutation has on GBM is through the mitogen activated protein kinase (MAPK) pathway (Fig 1.3). The distinction between the two methods of proliferative signalling is seen in the levels of invasion and migration of the cells, with MAPK alterations leading to the more diffuse GBM phenotypes ${ }^{33}$. This occurs because the MAPK pathway results in the 
activation of the transcription factors FOS and JUN, which have a role in cellular migration 34. Hence, over stimulating this pathway results in a more invasive tumour.

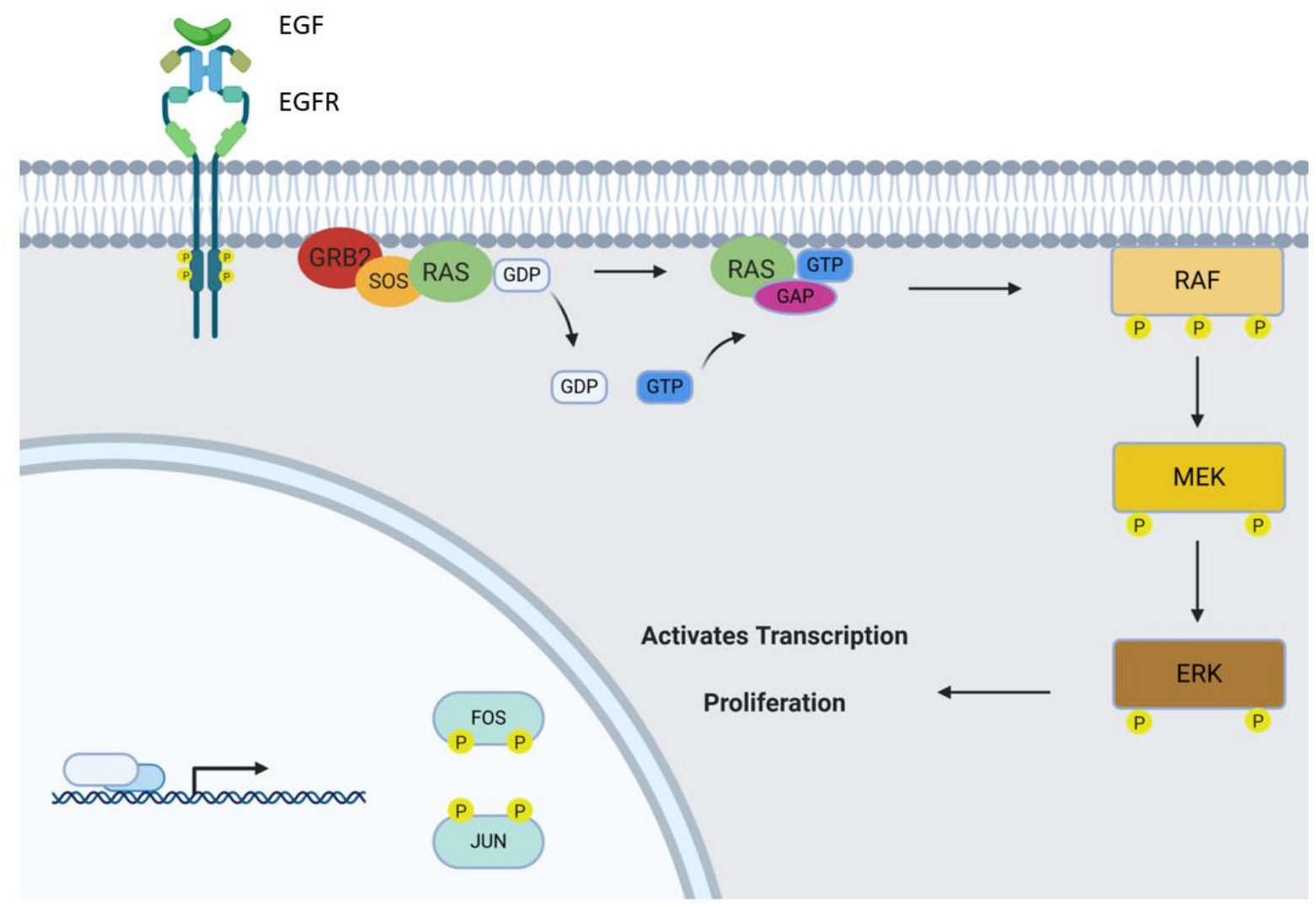

Figure 1.3: EGFR and MAPK signalling pathway. EGF binds to the extracellular domain of EGFR. Phosphorylation (yellow p) of the intracellular domain recruit's adaptor proteins and RAS-GDP. Once activated, Ras-GTP initiates the phosphorylation cascade of MAPK (RAF, MEK and ERK) which results in phosphorylation and activation of transcription factors FOS and JUN. These promote transcription (arrow on DNA) and initiate proliferation. Created with BioRender.

Mesenchymal tumours are named due to the presence of markers similar to that of an epithelial-mesenchymal transition, such as high expression of CHI3L1, MET and CD44 22,26. The defining driver mutation for this subtype is the loss of NF1, a CNS specific tumour suppressor gene ${ }^{22}$. NF1 is a GTPase activating protein (GAP) which converts RAS-GTP to RAS-GDP. As a result, NF1 inhibits RAS by removing its ability to phosphorylate and activate downstream kinases ${ }^{35}$. Through the loss of RAS inhibition, 
stimulation of both AKT/mTORC1 and MAPK pathways are increased. As in the classical subtype, this mutation is seen in co-occurrence with a PTEN deletion. The outcome of this co-deletion is the aggressive and invasive nature of the tumour. Additionally, the mesenchymal subtype shows the highest levels of suppressive immune cell infiltration and necrosis; this is believed to be due to the increase in NF-кB family proteins and TNF superfamily proteins ${ }^{36}$. This combination results in mesenchymal tumours having the worst survival rate of all the GBM subtypes, which is associated with these tumours acquiring the most treatment resistance post initial therapy 37

The proneural subtype is the most distinct of the classifications. The most frequent mutations are in the TP53 gene (referred to as P53); in particular loss of heterozygosity, which frequently occur in this subtype. In comparison, these P53 mutations are infrequent in the classical subtype, despite being the most frequently mutated gene in all cancer 22 . These tumours are also partially defined by the resemblance to oligodendrocytes rather than astrocytes ${ }^{22}$. There is a further subdivision seen in this subtype, with an almost mutually exclusive relationship between PDGFRA and IDH1 mutations. Similar in nature to EGFR, PDGFRA is another example of a tyrosine kinase receptor. The signature mutation to this gene is a focal amplification of chromosome $4 \mathrm{q} 12$, coupled with an increase in expression of PDGFRA. While the survival rate for proneural tumours is higher than in other subtypes, tumours with PDGFRA mutations show the lowest survival within the proneural subtype 22,38 .

IDH1 mutations make up the second component of the proneural subtype and tend to be secondary GBM tumours. The most common alteration to the protein is the point mutation of IDH1R132H 39. This is a gain of function mutation which impacts the metabolism of the tumour. Instead of converting isocitrate to $\alpha$-ketoglutarate ( $\alpha K G)$, the mutant IDH1 produces the oncometabolite D-2-hydroxyglutarate (D2HG). This oncometabolite competitively inhibits $\alpha \mathrm{KG}$ s interactions downstream ${ }^{40}$. Importantly in GBM, $\alpha K G$ is required for two DNA related events: histone demethylation and DNA demethylation. Histone methylation acts as a temporary marker of either transcriptional activation (H3K4Me3) or repression (H3K9Me3 and H3K27Me3) ${ }^{41}$. For histone methylation to be removed, histone demethylation complexes such as JMJD are required. 
This reaction requires $\alpha K G$ to be converted to succinate, without which demethylation is limited ${ }^{42}$ (Fig 1.4). As a result, the mutant IDH1 proteins introduce two effects: a decrease in $\alpha K G$ production and the competitive inhibition of $\alpha \mathrm{KG}$ via D2HG. Consequently, there is a significant decrease in demethylation of histones ${ }^{39}$. A similar mechanism is seen in the inhibition of DNA demethylation when IDH1 mutant proteins are present. This is believed to be through the TET family of enzyme, such as the 5-Methylcytosine hydroxylases, which are regulated by $\alpha \mathrm{KG}$ in the same manner as JMJD. These two mechanisms are theorised to be the early drivers of gliomagenesis for these tumours ${ }^{43}$. IDH1 mutants frequently possess a glioma CpG-island methylator phenotype (G-CIMP). This is a pattern of highly methylated DNA regions, also correlating with a decrease in transcriptional activity ${ }^{44}$.

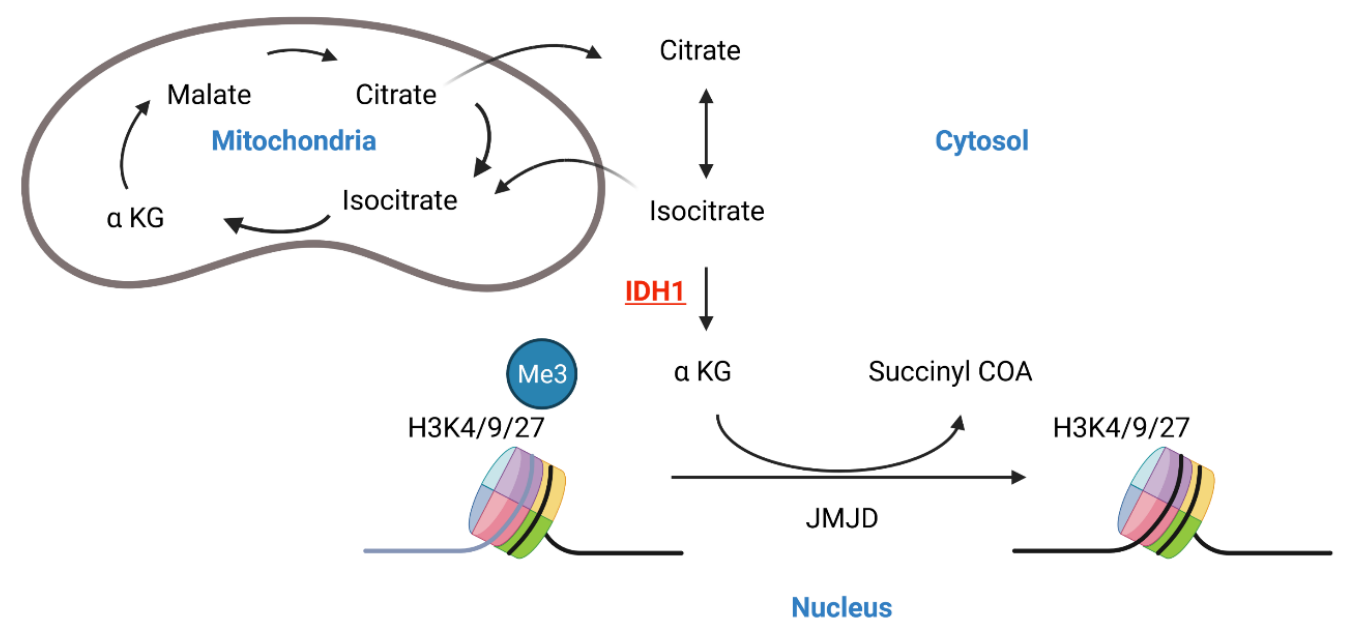

Figure 1.4: The interaction between IDH1 and histone demethylation. Citrate is transported out of the mitochondria via the CTP from the TCA cycle (abbreviation shown), to the cytosol. Cytosolic citrate is converted to isocitrate, which IDH1 (red) converts to $\alpha$ ketoglutarate $(\alpha K G)$. In the nucleus, $\alpha K G$ is required for JMJD to demethylate histones (H3K4, H3K9 and H3K27). Created with BioRender. 
Both of these methylation related effects result in the IDH1 mutant proneural tumours having a more 'differentiated' morphology due to the substantial decrease in transcriptional activity at these loci ${ }^{45}$. These mutated IDH1 proteins affect the progress of GBM in two contrasting mechanisms. Firstly, through the decrease in $\alpha K G$, there is a decrease in the tumours ability to produce ATP via glycolysis and oxidative phosphorylation. This combined with the significant decrease in transcriptional activity means that these tumours develop much more slowly than other GBM, and as a result have a slightly improved prognosis 22,43. However, despite this, the presence of D2HG is oncogenic and drives the tumoural progression. This is believed to be through an increased production of radical oxygen species (ROS), the presence of which in the cell induces DNA damage and results in chromosome instability ${ }^{39}$. This mechanism produces further tumorigenic mutations and develops the cancer. Overall, IDH1 mutant proneural tumours represent a population of GBM which is unique in its progression.

\subsubsection{Inter-tumoural heterogeneity and the cell of origin in glioblastoma}

One reason for these the inter-tumoural heterogeneity seen between tumours stems from the theory that GBM has many potential cells of origin. Unlike many tumours, GBM does not appear to develop from a single, consistent cell type ${ }^{45}$. While this has been known for a long time, hence the long form name, glioblastoma multiforme, there is no consensus on the exact cells involved. The subventricular zone (SVZ) is theorised as a strong candidate for a 'site of origin' of GBM ${ }^{46}$. This is because the SVZ is the location of neural stem cells (NSC), a multipotent stem cell which has a high capacity for self-renewal. NSCs from the SVZ lead to the development of three general cell types: neurons, oligodendrocyte precursor cells (OPC) and astrocytes ${ }^{45}$.

Previously, it was believed that all tumours developed through natural selection events, leading to a stochastic model where tumours acquired mutations, some of which increased fitness and others that decreased it. However, over time it has been seen that a small proportion of cells have the ability to initiate tumours, called cancer stem cells (CSC) 45. These are a sub-population of the solid tumour which, like normal stem cells, have the capacity for self-renewal. CSCs also possess any of the following characteristics: a) the 
ability to generate the heterogeneous collection of cancer cells in the solid tumour, b) resistance to chemo- and radiation therapies specifically used to target malignant tumours, and c) the ability to generate metastatic tumours 47,48 . This results in a cell which fulfils one of the 'hallmarks of cancer' defined by Hanahan and Weinberg (2000), that a cancer cell must have enabled immortality ${ }^{49,50}$. Because of the multipotent ability of cells in the SVZ, it has been theorised that cells in this region have the capacity to develop into CSCs ${ }^{46}$.

As NSCs are the most multipotent of the cell types present in the SVZ, it is commonly hypothesised that GBM is derived from these local stem cells. This is because for NSCs to develop into a CSC there are fewer driver mutations required to become cancerous, than are needed in more differentiated cells. As a result, these cells are more likely to gain further mutations to achieve the remaining hallmarks, such as provide selfgrowth signals in the form of mutated oncogenes or tumour suppressors. Additionally, the cell markers seen in the classical and mesenchymal subtypes, GFAP, Nestin and CD133, are highly expressed in NSCs 45,46,51. The limiting factor in this hypothesis is seen in the in vivo regeneration of NSCs. While theoretically NSCs have replicative immortality, in the brain individual NSCs do not actively divide multiple times ${ }^{52}$. Liu et al. (2011) induced NF1 and PTEN mutations in an in vitro NSC culture in the attempt to induce a tumorigenic phenotype. However, this resulted in the induction of a cell which had more resemblance with an oligodendrocyte than a CSC, and with these mutations did not show replicative immortality in vitro ${ }^{53}$. Because of this, it is currently debated whether NSCs possess the same stem-like capacity of other cells, such as haemopoietic and intestinal stem cells ${ }^{52}$. As a result, while NSCs would be capable of generating such a heterogeneous cancer as GBM, their low mitotic rate has led to doubt as to whether they are the sole source of GBM CSCs 45,46 .

Because of this debate, research has been conducted into whether other stem-like glial precursors in the brain are a likely candidate for a GBM CSC. Oligodendrocyte precursor cells (OPCs) are another potential cell of origin. While OPCs differentiate from NSCs in the SVZ, these cells still retain stem-like properties and have a significantly higher rate of replication than NSCs. Garcia-Marques (2014) showed that DNA-labelled OPCs have the capacity of dividing asymmetrically up to 400-times per cell. OPCs account for 
approximately $10 \%$ of the adult brain, by cellular mass. They are present in the SVZ but also migrate outwards after differentiation ${ }^{54}$. Because of their high abundance in the brain and their high turnover rate, OPCs are a strong candidate for CSCs in GBM ${ }^{46}$. Additionally, while NSCs share surface markers with classical and mesenchymal subtypes, proneural GBM show surface markers which are specific to oligodendrocyte development in particular OLIG2 and proteins from the SOX family ${ }^{22}$. This concept was confirmed by immunohistology data gathered by Motomura et al. (2012). While the subtypes were categorised by molecular and expressional phenotypes, there appears to be three histological groupings: OPC type, differentiated oligodendrocyte type and astrocyticmesenchymal. The overall survival for these groups mirrors that of the subtypes, with the astrocytic mesenchymal (mesenchymal) types showing the worst prognosis, and the oligodendrocyte type the greatest (proneural) ${ }^{55}$. Since the major determinant of a classical tumour is molecular (alterations to EGFR) and not histological, these are not comparable. While this does not confirm that the cell of origin for these tumours is the same as the histology would suggest, this data shows that the pathology of GBM is complex and not completely understood.

The final theory for the cellular origin of GBM is astrocytes. Astrocytes account for approximately half the mass in the adult brain ${ }^{2}$. This is largely due to the wide-ranging tasks which astrocytes are developed to conduct ${ }^{45}$. Similarly to NSCs, terminally differentiated astrocytes possess the cell surface markers of the classical and mesenchymal subtypes, and these two forms of GBM are said to be 'astrocyte-like' in the morphology of the cells ${ }^{22}$. However, while NSCs and OPCs have the potential to mutate into an initiating cell because of their stem-like potential, it is largely believed that astrocytes do not proliferate after terminal differentiation is reached ${ }^{3}$. But, as the depth of knowledge surrounding astrocytes increases, their potential as a candidate origin for GBM does too. Certain reactive astrocytes possess the ability to proliferate in response to stress; either because of immunological or structural changes in the brain ${ }^{3,56}$. Multiple in vitro studies have been able to manipulate astrocytes into a more stem-like phenotype ${ }^{57-59}$. Vitucci et al. (2017) showed through silencing Trp53 and Nf1 in murine astrocytes, a mesenchymal-like cell was induced ${ }^{58}$. However, it is not generally hypothesised that astrocytes develop into CSCs in vivo as the number of mutations required is far more than would be in NSCs or 
OPCs ${ }^{46}$. Nevertheless, because of the variation in the tasks performed by astrocytes, the morphological and cellular diversity means that astrocytes are very likely to play a role in gliomagenesis. However, it is unknown whether this is as a cell of origin or in facilitating the tumour microenvironment where GBM can develop.

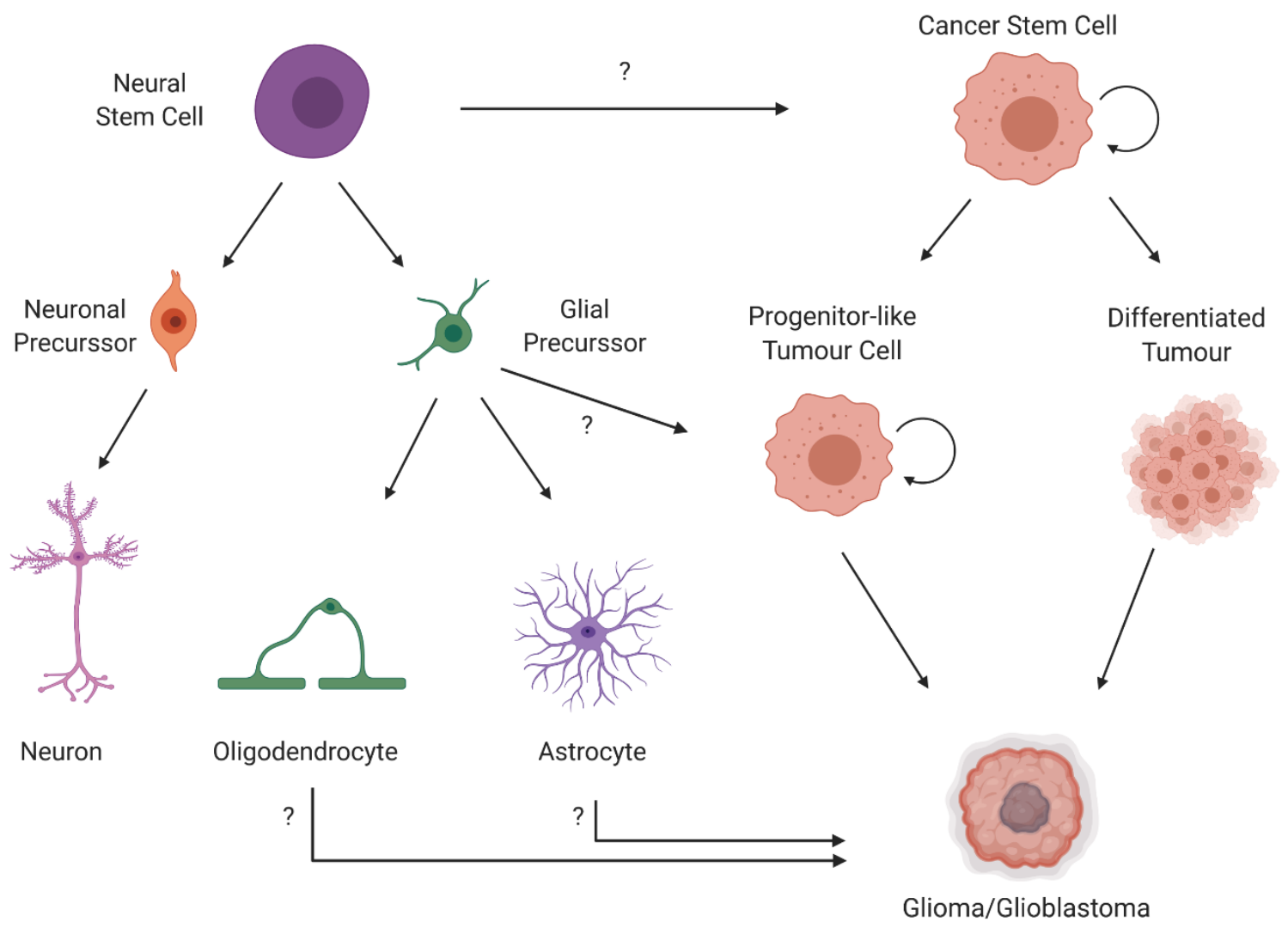

Figure 1.5: Candidates for GBM cell of origin. CNS cells are shown in relation to the theories regarding which cells are predicted to initiate GBM pathology. Unknown links (?) and self-renewal (circular arrow) show the progression to GBM. Created with BioRender.

\subsubsection{Intratumoural heterogeneity}

The GBM subtypes are a useful method of grouping mutation patterns and potentially understanding the nature of each tumour's progression. However, in the years following Verhaak et al. (2010), it was observed that the mutations seen in each patient are 
more fluid and dynamic than previously believed. Patel et al. (2014) used single-cell RNASeq to show that transcriptomes of all the subtypes can be seen within individual tumours 60. While most tumours still possess a dominant subtype - for example mesenchymal GBM are categorised as such because of their highly inflammatory nature in the gross pathology - there are many mutational events which lead to the final tumour. It is because of this intra-tumoural or cellular heterogeneity that treatment development for GBM has not advanced as much as other cancers over the past three decades.

A key example of how intratumoural heterogeneity contributes to GBM progression is through treatment resistance. As mentioned previously (1.1.3), approximately $50 \%$ of GBM tumours contain cells with epigenetic MGMT mutations, which silence the genes transcription. Since only half have MGMT expressed, there is a heavy reliance on the alternative forms of treatment: surgery and radiation. However, because of the invasive nature of GBM, not all tumour tissue can be excised or killed in these procedures. This is important because the composition of GBM tumours have been shown to change throughout a treatment regime. Wang et al. (2017) showed that in treated IDH1 wildtype proneural tumours, the abundance of cells with NF1 mutations or deletions increased at recurrence. A small proportion of cells in the tumour had NF1 alterations at the original surgery. After tumour recurrence, this proportion was larger, and the tumours were subtyped as mesenchymal. This result showed that GBM tumours are not only heterogeneous, but also that the cellular make up is fluid. A key finding in this investigation saw that as cells with NF1 mutations increased, so too did the presence of tumour associated macrophages (TAMs). These TAMs are 'pro-tumour' and suppress the immune response against the cancer ${ }^{61}$. This result showed that the mesenchymal-related mutation of NF1 is directly correlated with the increase in immune infiltration.

In a study conducted by Lee et al (2018) used deep whole exome sequencing (WES) to compare a) tumour tissue, b) healthy brain tissue (or blood), and c) healthy tissue neighbouring the tumour, of GBM patients. It was found that there were low levels of driver mutations present in the healthy tissue adjacent to the tumour which were not present in the control tissue. Approximately 1\% of the mutational makeup of the tumour mass was seen in the surrounding, 'healthy', tissue. These results show that in GBM, not 
only do tumour cells invade into the parenchyma, but also tissue which appears healthy and would not be dissected away contains signs of early tumorigenesis ${ }^{62}$. This data implies that the development of GBM is substantially more complicated than previously thought and that the methods currently available to study the cancer are not able to accurately depict this complexity.

Research into the molecular and histological composition of GBM is consistently providing novel information which develops our knowledge in the field. Unfortunately, this knowledge alone has not been able to correlate with any progress in treating the tumour. The fact remains that while we are able to investigate the mutational landscape of GBM, a larger effort must be made into developing treatments which are more effective than TMZ and radiation. For this to happen, the development of more accurate models is required.

\subsection{Animal Models and Glioblastoma}

\subsubsection{The use of models in glioblastoma}

As with other forms of cancer, glioblastoma requires the use of models to test theories and research. These models are either entirely cell line based, for in vitro studies, or use animal models, for in vivo studies ${ }^{63,64}$. Glioblastoma cell lines are readily used around the world and possess many advantages, including decreasing the cost and time spent on experiments. There is no limit to what in vitro experiments can investigate, from simply the transcriptome of a GBM cell line ${ }^{65}$, to complex collagen flasks which aim to model the extra cellular matrix ${ }^{66}$. However, a major issue with in vitro studies is the limited ability for the research to translate to the organismal level, as true cell-to-cell interactions of CNS cells are extremely difficult to model outside the body ${ }^{66}$. Because of this, animal models are a major part of GBM research ${ }^{67}$.

Many aspects need to be considered when animal models are used in the experimental design. Mice tend to be the model of choice largely due to their size and 
relatively cheap maintenance. However, the murine physiology, especially the brain, is particularly dissimilar to that of human ${ }^{68}$. Because of this, GBM studies also utilise models ranging from rats to zebra-fish in order to utilise varying brain structures ${ }^{63,69,70}$. Regardless of which animal is used, however, when designing any animal model study, there are two options to consider: transplantation or genetically engineered models.

\subsubsection{Transplantation models in GBM}

The most frequently used method for modelling glioblastoma is through tissue transplantation 66,67. These models involve cells with tumorigenic capacity that are transplanted into a host organism. Once injected, tumours are fast to develop and can be used for a wide range of in vivo experiments; in particular, transplantation models are used for treatment development and biological validation studies ${ }^{67}$. There are many options and methods which are utilised in these models. If a tumour has been generated in your animal of choice, it can be cultured and transplanted into the same species. This is known as an allograft. Alternatively, if human tissue is used the concept is called a xenograft. The greater difficulty arises in choosing the location of the transplantation; as an orthotopic transplant at the site of interest (the brain) or subcutaneously (heterotopic). While the brain is more

biologically relevant, numerous studies opt for heterotopic transplantations when conducting treatment investigations ${ }^{67}$. This is because of the continuous difficulty in developing or finding new pharmaceuticals which are capable of passing the BBB, as a result, before optimising for CNS delivery, heterotopic transplants are the most viable option ${ }^{71}$.

Human GBM is a highly used source for transplantation models. The most frequently used form of this is human GBM cell lines; examples of these are U87-MG and LN18 ${ }^{72}$. These are cell lines which have been derived from GBM tumours and cultured over time. The mutations present in these lines are known to have induced the development of GBM. Over the decades of culturing, these cell populations have become more homogeneous than when first produced. However, because they are extremely accessibile, GBM cell lines are a very common source of tissue for GBM transplantation models ${ }^{64}$. 
Alternatively, patient derived xenografts (PDX) are a common source of cells to be transplanted. These PDXs are samples of human GBM tissue sectioned from surgery which are then injected into the host to grow. As these tumours are known to possess an array of driver mutations that directly result in GBM formation, PDX transplantation is the most biologically relevant model available. This means that studies which use PDX as their model can choose the subtype, methylation subtype (G-CIMP and MGMT), and whether the tumour had recurred or not ${ }^{73}$. This allows the research to control as many variables as possible while still portraying the tumour accurately. An example of this can be seen in Ulasov et al. (2019). This study utilised the array of PDX models available to investigate the role of TMZ on the tumours transcriptional rate. It was concluded that over all the subtypes, TMZ treatment has the capacity to regulate the 'stemness' of GBM tumours ${ }^{74}$. Through using multiple known PDX models, which have had their genome profiled and the patient history associated with the tissue, the animal model is able to be more relevant to the human disease. This human relevance is extremely important in relation to drug testing. Stackhouse et al. (2019) posited a potential novel method of screening multiple drugs which used NanoString technology to analyse the gene expression after sensitisation. This investigation utilised 11 PDX samples and found 16 different expression signatures with these models ${ }^{75}$. The use of PDX introduces the possibility of personalised models. By using tissue from patients' tumours, treatments are able to be tested before being chosen clinically ${ }^{76}$. This concept, while not in use, acknowledges the fact that each GBM tumour is different and that not all treatments will result in the same outcome in every situation. 


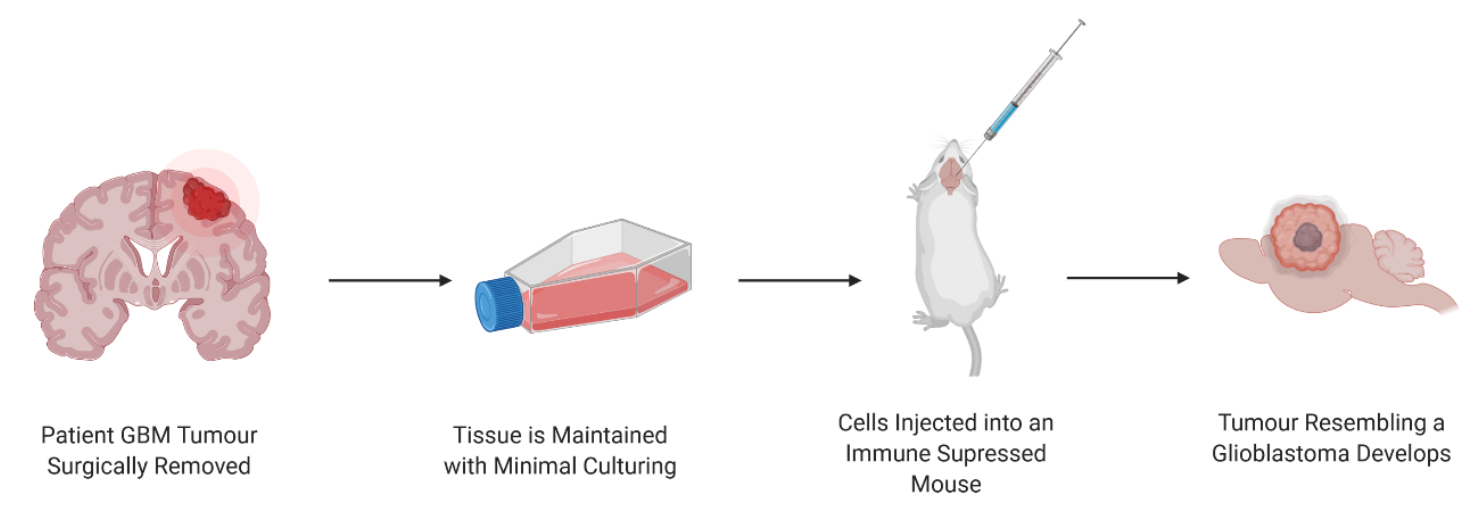

Figure 1.6: Patient derived xenograft (PDX) transplantation design. Created with BioRender.

One alternative to transplanting human tissue is to utilise murine derived glioma cell lines. This is a very frequently chosen method of modelling all forms of GBM, because of the ease of accessibility to the cell lines and the much lower cost. The cell lines tend to be immortalised and therefore do not have a continued cost to purchase, unlike the PDX. These cell lines are generally derived from a chemically induced tumour in the desired tissue 64,67. GL261 is a very commonly used glioma cell line and was developed using 3methylcholanthrene; this is a carcinogen which interferes with DNA synthesis leading to mutations ${ }^{77}$. The main driver mutations in GL261 are a K-Ras mutation and a Trp53 point mutation. As this cell line is of mouse origin, and still maintains enough 'self antigen, there is no immediate immune response against the transplantation and a fully immune competent $\mathrm{C} 57 \mathrm{BL} / 6$ host can be used as a model ${ }^{67}$. These models are also very well characterised; many studies have published important aspects of GL261, such as their response to treatment ${ }^{78}$, and their transcriptome ${ }^{79,80}$. The benefit of this is that studies using murine cell lines are often more replicable between lab groups, this is simply because the same cell lines have been used for decades. Overall, GL261 represents the most generally utilised transplantation model of a brain cancer because of reliability in tumour growth and consistency in the data produced ${ }^{81}$. 


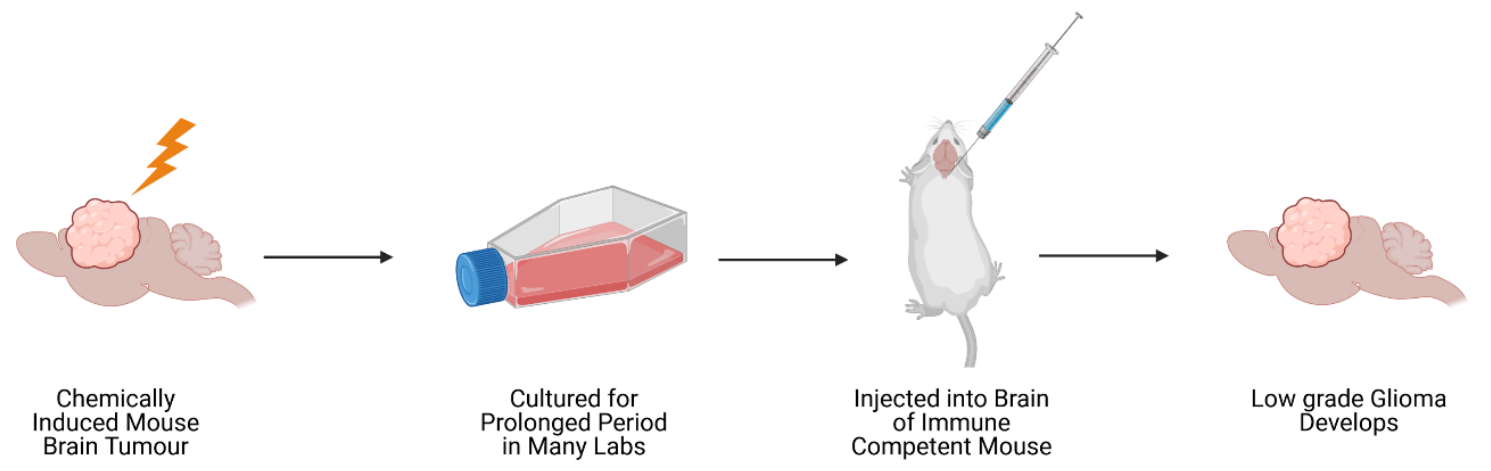

Figure 1.7: Murine cell line transplantation design. Created with BioRender.

\subsubsection{Limitations of the current transplantation models in GBM research}

As discussed previously, GBM research has not been able to make any comprehensive discoveries using the current models available. Despite their presence as the most common models in the field, transplantation models have many limitations ${ }^{67}$. The major issue with PDX models is the limitations of transplanting human tissue into a mouse model. As the cells must be transplanted into another species, if the immune system is active there would be a graft versus host response against the PDX ${ }^{63}$. To counter this, PDX models must have a severely immune suppressed host: either the athymic 'nude' mouse or the NOD-SCID $\gamma$ are selected ${ }^{67}$. This is the major limitation of the model, as a large component of the tumour microenvironment is the infiltration of immune cells 66,81 . This is a large problem when it comes to representing the mesenchymal subtype, which has the worst prognosis due to the increased immune component ${ }^{22}$. As a result, PDX models of GBM are unable to represent the tumour completely and accurately.

These limitations are exacerbated in the human GBM cell lines. Because of the decades of culturing in different labs, there cell lines are too homogeneous, and genetically and transcriptomically diverged to adequately represent GBM when transplanted. Lee et 
al. (2006) showed that GBM cell lines no longer adequately represent the human tumour. While these lines do form tumours in vivo, they do not show the appropriate array of CSC characteristics, such as self-renewal, and do not have the transcriptome seen in GBM. This is thought to be due to generations of culturing in standard conditions, rather than in stem cell media ${ }^{82}$. This result is corroborated by Allen et al. (2016), who conducted research in the same lab which the U87-MG cells were developed. This study showed that the U87MG cell lines which are distributed for research today are significantly different from the original cells produced in $1968^{83}$. In addition to this, human GBM cell lines possess the same limitation that is seen in PDX, as an immune suppressed mouse must be used as the host.

While murine cell lines address the limitation of immune rejection, there are other problems which are encountered. The major issue of the widely used GL261 model is that it resembles an LGG and not GBM. These cell lines form relatively uniform tumours with distinct borders, whereas one of the key features of GBM are the very diffuse borders with pseudopalisading necrosis ${ }^{81}$. Even though these tumours develop extremely rapidly - mice reach terminal stages within four weeks - the tumours that develop have not been useful in answering biological questions about GBM ${ }^{81}$. This is largely due to the lack of diversity a cell line like GL261 contains. As these cells were derived from a non-GBM tumour in a mouse, the mutations present are not related to cancer in humans.

The inability to customise is a shared limitation that transplantation models possess. The same model and experimental designs are unlikely to always be suited to every research question. Because of this, a key feature in GBM models must be the ability for the model to match the purpose of the research. GL261 cell lines are able to be genetically altered, however, the key driver mutations present - K-Ras and Trp53 (homolog of P53) - are not a frequently seen pair of mutations in GBM 22,26,27. While the ability to acquire PDX samples which are suited to your investigation is useful, it is limited heavily by the ability to acquire a large enough variety in the tissue samples available ${ }^{64}$. Although it is a benefit that the mutations seen in the PDX are directly from a tumour and known to result in GBM development, customisation is a much needed aspect in developing a model, and is not currently seen in transplantation studies. 


\subsubsection{Genetically Engineered Mouse Models}

Using genetic editing tools, innovations have been made in creating genetically engineered mouse models (GEMMs) of GBM ${ }^{63,70 . ~ T h e s e ~ m o d e l s ~ u t i l i s e ~ t h e ~ r a p i d l y ~}$ developing depth of genomic knowledge available. Mutations known to contribute specifically to GBM can be introduced to the brain tissue of mice, so that the host's own tissue will develop into a tumour. As with the transplantation of murine cell lines, this method has the advantage of utilising fully immune competent mice ${ }^{84}$. However, unlike murine cell lines, GEMMs are more relevant to GBM because of the carefully curated mutations. This ability to customise GEMMs based on the available genomic data is a clear advantage over transplantation models. While the TCGA data available has shown the depth of genetic and molecular alterations that GBM possess, it has not given us further information on how these changes have driven the tumour progression, or what is necessary to stop it. This limitation is seen with PDX models; while the tumours are genuine GBM, we do not necessarily gain further biological knowledge through their use. Contrastingly, GEMMs allow for researchers to use the genomic data available and develop experimental designs which lead to a deeper understanding of the disease.

\subsubsection{Conditional mutation GEMMs}

The most frequently used method to develop GEMMs has been to use a conditional knockout of the genes of interest ${ }^{85}$. This is generally achieved through the Cre/LoxP system. This involves the gene to be deleted being flanked by LoxP digestion sites in the germline of one mouse strain, and in another strain the recombinase, Cre, is conditionally expressed in the tissue where you wish to grow the tumour. For example, if $\operatorname{Tr} 553$ (referred to as P53) were to be deleted in astrocytes, one strain would have the P53 flanked by LoxP and the other strain would have Cre conditionally expressed in tissue which expressed an astrocytic marker, such as GFAP. After crossing these mice, in astrocyte tissue only, Cre would be expressed and LoxP sites would be present. As a result, Cre would cut both LoxP sites, and the sequence between will be deleted. The result of this cross would be an 
offspring which has had the P53 gene conditionally knocked out in astrocytes. This model is extremely robust and reliable, and has been used for the past three decades ${ }^{63,67}$.

Through the introduction of a template strand of DNA, which can be expressed alongside Cre, conditional knock-in mice can be created as easily. The tumours which form are known to be due to the mutations introduced, therefore these models are very useful in terms of understanding how GBM mutations are responsible for particular aspects of tumorigenesis ${ }^{85}$. For example, Bardella et al. (2016) studied the effect the IDH1R132H mutation had on the SVZ. The point mutation was introduced to the endogenous Idh1 gene in OPCs, using the Nestin marker to express Cre. The result of this experiment was the discovery that the $I D H 1^{\mathrm{R} 132 \mathrm{H}}$ mutation and the production of $\mathrm{D} 2 \mathrm{HG}$ are sufficient to induce an LGG but not GBM ${ }^{86}$. This type of simple experiment is not possible with the current transplantation models, and its result expands the depth of knowledge of GBM. 


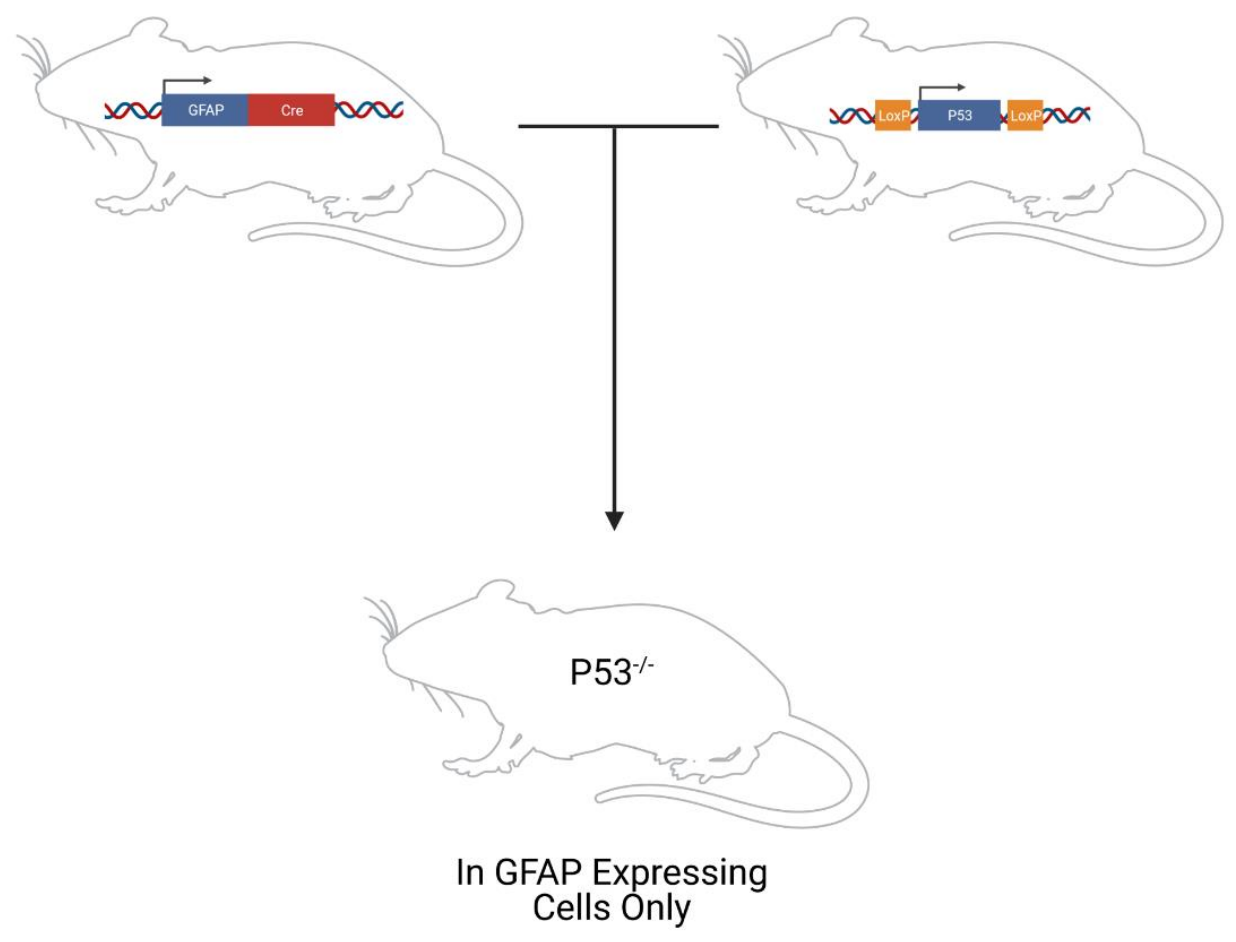

Figure 1.8: Conditional knockout of P53 via Cre/LoxP design. Two parental mice are crossed; one with Cre expressed conditionally in cells expressing GFAP and another with P53 surrounded by LoxP sites. The offspring would be P53 - in $\mathrm{GFAP}^{+}$tissue. Created with BioRender.

However, Cre/LoxP models are expensive to develop and maintain, as entire strains must be purchased and bred. If these models are used for an investigation, generally mice with the correct Cre and LoxP sites will be purchased and then bred for as long as they are needed. This is an added expense compared with transplantation models which only require a single line to be kept alive. As murine cell line experiments only require wildtype mice, the decision to use conditional knockout mice must be weighed against this option. 


\subsubsection{CRISPR/Cas}

More novel methods of developing GEMMs have been created throughout the $21^{\text {st }}$ century. One of the most utilised has been the CRISPR/Cas system. This technology uses a short guide RNA ( $\operatorname{sg} \mathrm{RNA}$ ) sequence which is specific to your gene of interest to direct an endonuclease, Cas, to cut the genome. The specificity of this cut is enhanced because the Cas9 protein can only cut when the sgRNA is followed by a protospacer adjacent motif (PAM) site, NGG ${ }^{87,88}$. Following this, the cell will initiate one of two mechanisms to repair the DNA: a) non-homologous end joining (NHEJ) or b) homology directed repair (HDR). NHEJ is the most frequent pathway activated when dsDNA cuts are made to the genome 89,90. This involves the cut ends being trimmed back and then re-joined in a non-sequence specific manner. This process is rushed and inaccurate, as a result there will be insertion or deletion mutations (indels) introduced at the cut site. This is the method in which CRISPR/Cas tends to be used to knockout genes. However, by altering the method of cutting, HDR can be influenced ${ }^{91}$. Altered Cas proteins can introduce ssDNA breaks, which preferentially select for HDR. This pathway recruits the homologous allele, which is used as a template to fix the DNA. This technique is used to change a heterozygous mutation into a homozygous one. Alternatively, through providing a custom template alongside the CRISPR/Cas machinery, a knock in mutation can be forced by incorporation of the template instead of the homologous strand ${ }^{89-91 .}$ 


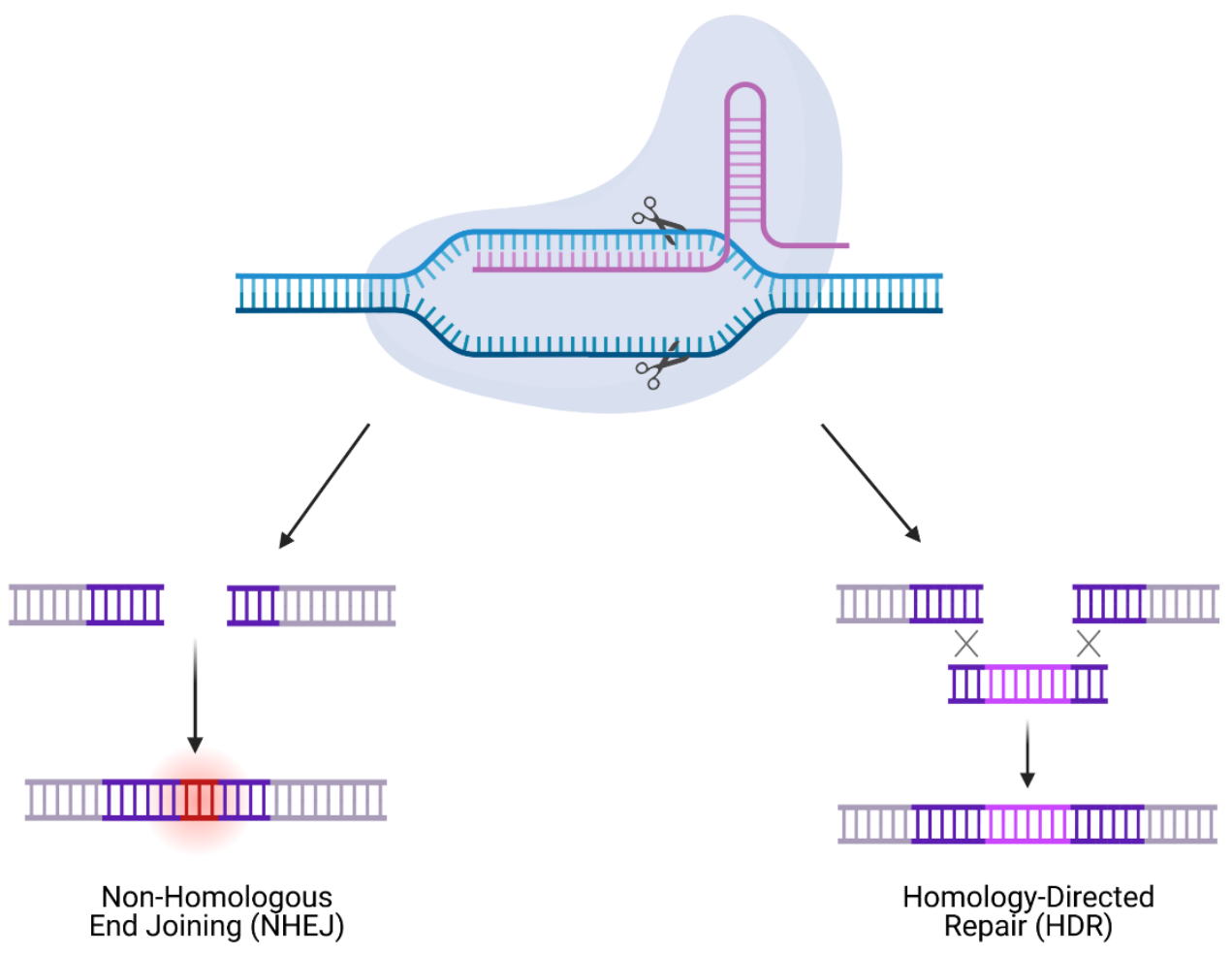

Figure 1.9: CRISPR/Cas9 mutations. Cas9 (round) and sgRNA (pink) combine and bind to the gDNA (blue and black). Cuts are made to both strands. Insertion or deletions (red) are induced via NHEJ. Template or homologous sequence (light pink) are incorporated in HDR. Created with BioRender.

\subsubsection{CRISPR/Cas and GEMMs in glioblastoma}

GEMMs developed from CRISPR/Cas are much faster than conditional knockouts as mutations can be introduced directly to the mouse rather than through breeding ${ }^{92}$. The mutations can also be introduced in a cell-conditional manner. By creating a lentivirus which contains the sgRNA, Cas protein and surface receptor specific to the cell of interest, CRISPR mutations can be specifically given to particular cells ${ }^{92-94}$. Using the example from before, with a sgRNA specific to P53, a lentivirus can be targeted specifically to cells 
expressing GFAP. The result of this would be a mutation to P53 astrocytes without the time expense of developing a litter.

An area of GBM research which has benefitted from CRISPR is immunotherapy. Using GL261 transplantations, studies can investigate how the adaptive immune system can be recruited to target the tumour. Ye et al. (2019) used a novel transposon-based approach to introduce CRISPR/Cas mutations to mouse $\mathrm{T}$ cells. Using an adenoassociated virus (AAV) as the vector, a library of $\operatorname{sgRNA}$ were tested in vitro for their effect on targeting cancer cells. Through this exploratory experimental design, they discovered four genes which have anti-tumour effects. Pdia3 knockouts showed the greatest levels of mouse survival when tested in vivo after intravenous delivery of the AAV ${ }^{95}$. However, while this is a novel use of genetic modification to increase our knowledge of GBM, the study remains limited due to the lack of resemblance GL261 has to GBM.

One of the hurdles in creating GBM-specific CRISPR-induced GEMMs is the same limitation which holds back treatment development, the blood-brain barrier. Numerous studies have been able to effectively induce non-brain tumours in vivo through introducing CRISPR viruses to mice, either directly to the organ or through the blood. Xue et al. (2014) induced Pten and P53 mutations in vivo to hepatocytes through hydrodynamic tail vein injections of a plasmid, resulting in GEM models of liver cancer ${ }^{96}$. However, studies have not been able to induce orthotopic GBM-like tumours in vivo to mature mice through this technique. Zuckermann et al. (2015) successfully introduced knockouts of Pten, Nf1 and P53 via in utero electroporation when targeting the forebrain of the embryo ${ }^{97}$. Despite this success, this technique is not as efficient as Cre/LoxP in inducing brain targeted GEMMs and is currently very infrequently used to develop GBM knockout models.

There are two further limitations which have yet to be addressed in the field of CRISPR research in GBM: a) introducing the complexity and heterogeneity seen in the human tumour, and b) the high cost to develop models which produce statistically meaningful data compared with transplantation models. The first of these issues cannot be fixed without advances in genetic editing. It is currently not possible to introduce the amount of mutations seen in GBM in vivo; largely because of the intratumoural heterogeneity, with many cells within the tumour containing differing mutations. The 
second issue will inevitably be improved as technology becomes cheaper over time. However, because of the expense, research currently cannot produce highly powered statistical data from GEMMs as efficiently as is possible with transplantation. The positive which can be taken from the gene editing field is that in vitro mutations are much easier to implement and significantly cheaper than in vivo.

\subsection{Aims of the Study}

\subsubsection{A Customisable Cell Line Model of Glioblastoma}

Currently there is a major gap in glioblastoma research. Transplantation models are an excellent source of in vivo tumours and are currently used readily. However, when it comes to accurately representing the tumour, these models fall short; either through the absence of immune infiltration seen with PDX or the complete lack of GBM complexity in murine cell lines. While these limitations are addressed to a point in GEM models, the concept is essentially held short by the technology's inability to produce in vivo mutations which fully represent GBM in a cost-effective manner. This leaves a gap in the research where gene editing has the potential to aid, rather than replace, transplantation models.

The aim of this project is to take the genomic data known about GBM and produce a model which addresses the limitations which currently hold back research in the field. The goal is to make a $\boldsymbol{G}$ enetically $\boldsymbol{E}$ nineered $\boldsymbol{M}$ ouse $\boldsymbol{C}$ ell $\boldsymbol{L}$ in $\boldsymbol{C}$ Model (GEM-CLeM) which is customisable and adaptable to any GBM research topic. The base of this approach is an immortalised astrocyte cell line which expresses an EGFP transgene. This cell line was derived in the McConnell lab by Remy Schneider, from cortical and hippocampal tissue of an EGFP C57BL/ 6 mouse 98 and cultured until immortality was achieved ${ }^{99}$ (Fig 1.10). A key feature of this cell line is that while 'tumorigenic' mutations have been acquired, such as unlimited replicative ability, the cells are not transformed and cannot grow into a tumour in vivo. As a result, this cell line lends is the perfect base on which to introduce GBM specific mutations and create a transplantation model capable of use in immune competent mice. 


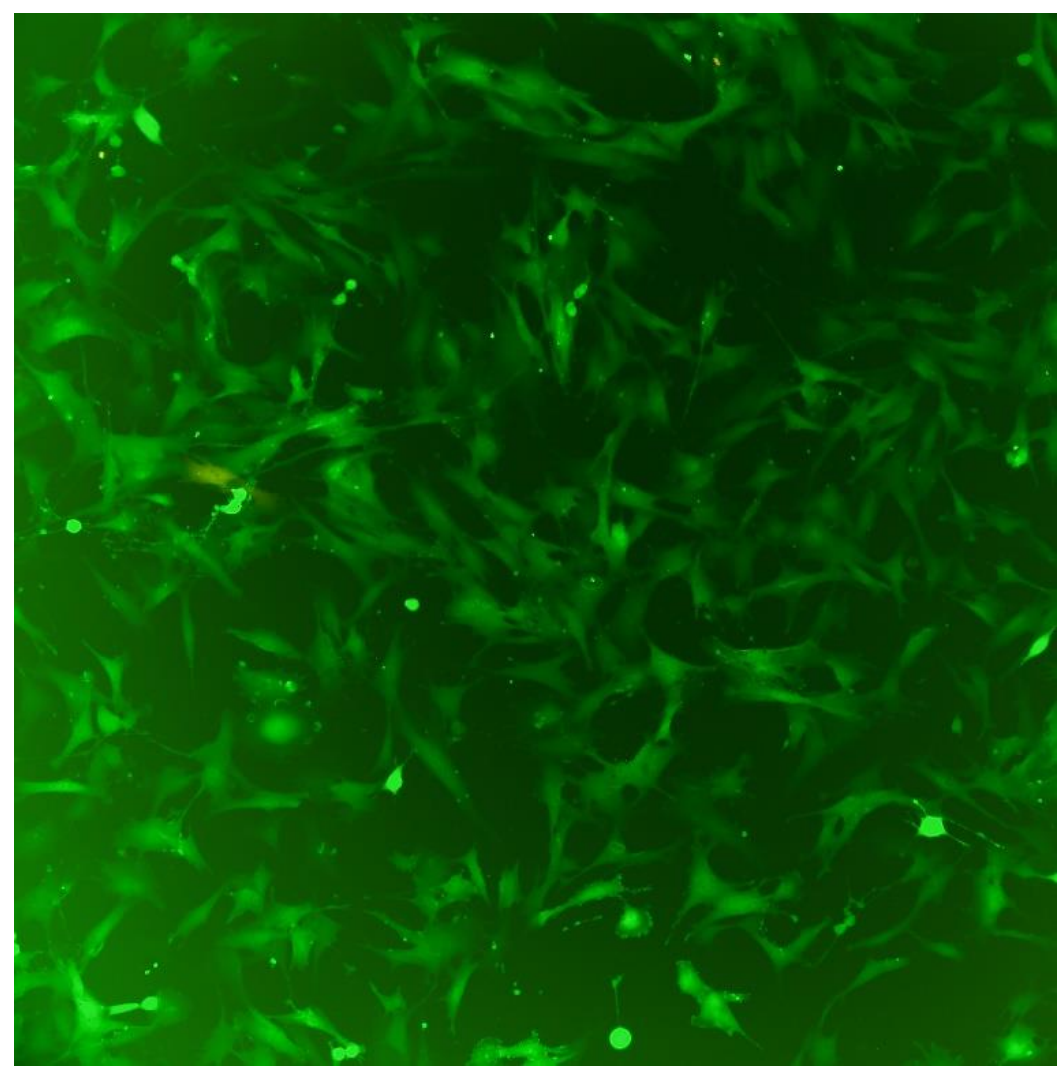

Figure 1.10: EGFP C57 astrocytes. Fluorescent microscope image with FITC filter.

Because of the time constraints on a master's project, the aim of this thesis was to develop and characterise the models in vitro, allowing for future studies to investigate the in vivo work. Two types of cell lines were proposed, a 'classical-like' and a 'secondary-like'; I chose tumour suppressors PTEN and P53 as the initial mutations to make these models, respectively. The protocols established in Xue et al. (2014) to develop CRISPR-mediated knockouts of Pten and P53 were optimised for these in vitro mutations, and the plasmids were gifted by Tyler Jacks for use in this project ${ }^{96}$. For the 'classical-like' model, the constitutively activated MAPK pathway enzymes, EGFRVIII and H-RAS V12, were chosen as the candidate oncogenes to be stably transfected on top of the Pten deletion. I 
chose IDH1 ${ }^{\mathrm{R} 132 \mathrm{H}}$ as the sole oncogene to transfect alongside the P53 mutation as this is the major clinically relevant signature of a secondary GBM tumour 22,39,100.

\subsubsection{Objectives of the study}

1. To develop tumour suppressor (Pten and P53) knockout astrocyte cell lines using CRISPR/Cas9

2. To stably transfect oncogene overexpression plasmids (EGFRVIII, RAS V12 and IDH1 ${ }^{\mathrm{R} 132 \mathrm{H}}$ )

3. To develop screening protocols for the introduced mutations

4. To characterise the developed cell lines in relation to the relevant GBM subtypes

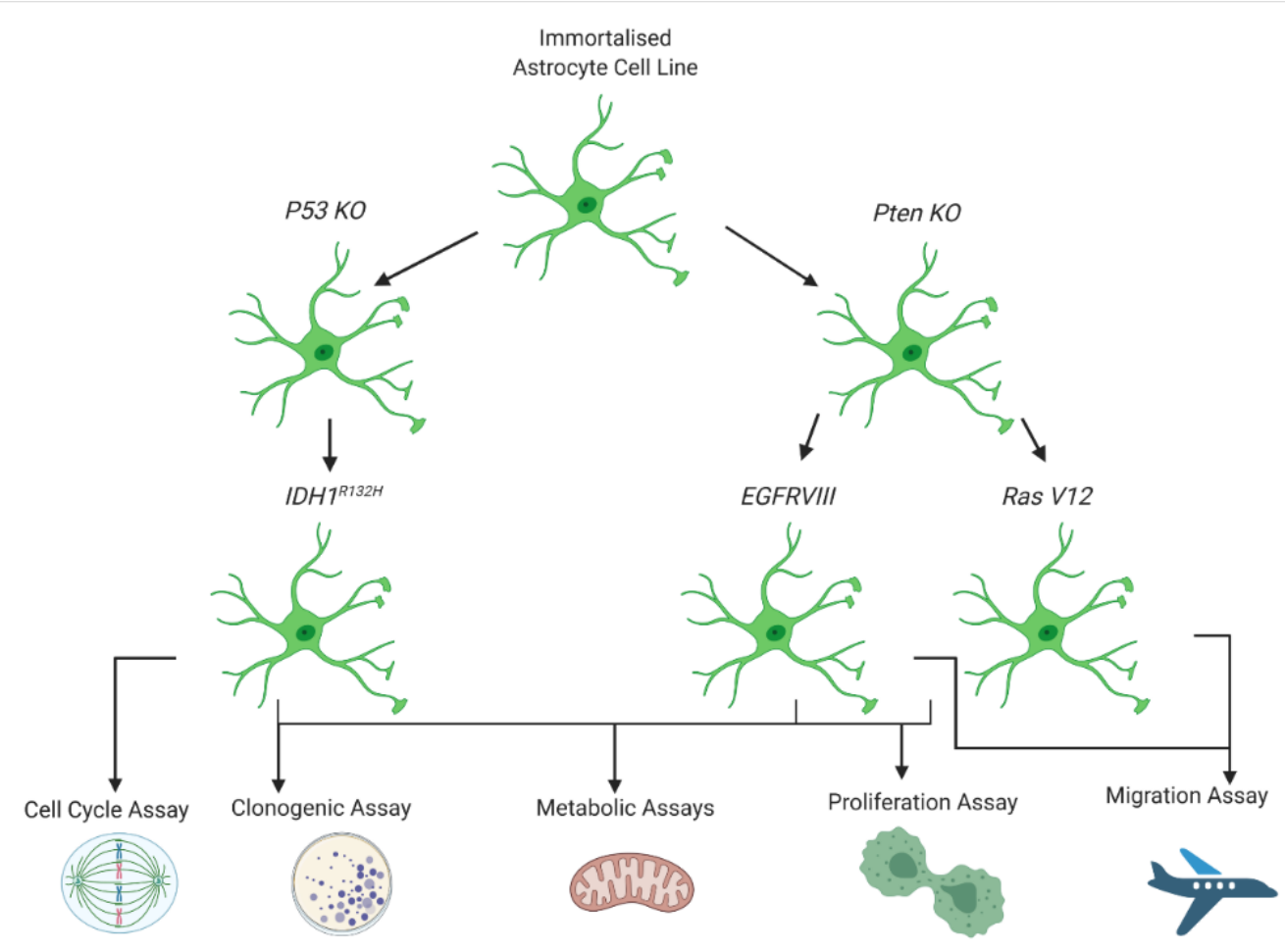

Figure 1.11: Graphical abstract for the project design. Created with BioRender. 


\subsection{Materials and Methods}

\subsection{Materials}

\subsubsection{Cell culture}

\begin{tabular}{|l|c|c|}
\hline Reagent & Catalogue No. & Company \\
\hline T75 Flask & COR430641 & Corning \\
\hline T25 Flask & COR430639 & Corning \\
\hline $\begin{array}{l}\text { Dulbecco's Modified Eagle Media } \\
\text { (DMEM) }\end{array}$ & 10313039 & Thermo Fisher Scientific \\
\hline $\begin{array}{l}\text { Dulbecco's Phosphate Buffered Saline } \\
\text { (DPBS) }\end{array}$ & 14190250 & Thermo Fisher Scientific \\
\hline Trypsin-EDTA & 25300062 & Thermo Fisher Scientific \\
\hline Glutamax ${ }^{\circledR}$ & 35050061 & Thermo Fisher Scientific \\
\hline Foetal Bovine Serum (FBS) & 15140122 & Thermo Fisher Scientific \\
\hline Penicillin-Streptomycin & 10131035 & Thermo Fisher Scientific \\
\hline G418 Sulfate & COR3516 & Corning \\
\hline Costar 6-well plates & COR3513 & Corning \\
\hline Costar 12-well plates & Corning \\
\hline Costar 24-well plates & & \\
\hline
\end{tabular}




\begin{tabular}{|l|c|c|}
\hline Costar 48-well plates & COR3548 & Corning \\
\hline Costar Flat bottom 96-well plates & COR3599 & Corning \\
\hline ViaFect $^{\text {TM }}$ Transfection Reagent & PME4981 & Promega \\
\hline CellTitre 96 AQueous $^{\text {TM }}$ & PMG3580 & Promega \\
\hline
\end{tabular}

\subsubsection{DNA}

\begin{tabular}{|l|c|c|}
\hline Reagent & Catalogue No. & Company \\
\hline Platinum SuperFi ${ }^{\circledR}$ PCR Mastermix & 12358050 & Thermo Fisher Scientific \\
\hline Surveyor ${ }^{\circledR}$ Mutation Detection Kit & 706020 & Integrated DNA Technologies \\
\hline Zymo Clean and Concentrate ${ }^{\circledR}$ Kit & D4004 & Zymo Research \\
\hline Zymo Quick-gDNA ${ }^{\circledR}$ MiniPrep Kit & D3025 & Zymo Research \\
\hline HyAgarose ${ }^{\text {TM } L E ~ A g a r o s e ~}$ & R9012LE & HydraGene \\
\hline SYBR ${ }^{\circledR}$ Safe DNA Gel Stain & S33102 & Thermo Fisher Scientific \\
\hline Orange G Loading Dye & O375625G & Sigma-Aldrich \\
\hline $30 \%$ Acrylamide/Bis Solution & 1610156 & Bio-Rad \\
\hline TEMED & 1610800 & EPio-Rad \\
\hline Ammonium Persulfate & 76322 & USB/Affymetrix \\
\hline
\end{tabular}




\subsubsection{Plasmid preparation}

\begin{tabular}{|l|c|c|}
\hline Reagent & Catalogue No. & Company \\
\hline NucleoBond Xtra Maxi Plus & MN740416 & Macherey-Nagel \\
\hline Luria Broth Base & 12795027 & Thermo Fisher Scientific \\
\hline Terrific Broth & 22711022 & Thermo Fisher Scientific \\
\hline Ampicillin (sodium salt, irradiated) & 11593027 & Thermo Fisher Scientific \\
\hline Monarch Plasmid Miniprep Kit & T1010S & New England Biolabs \\
\hline
\end{tabular}

\subsubsection{Protein}

\begin{tabular}{|l|c|c|}
\hline Reagent & Catalogue No. & Company \\
\hline Halt ${ }^{\text {TM }}$ Protease Inbibitor Cocktail & 78429 & Thermo Fisher Scientific \\
\hline Qubit ${ }^{\mathbb{P}}$ Protein Assay Kit & Q33212 & Thermo Fisher Scientific \\
\hline $\begin{array}{l}\text { PVDF membrane for protein blotting (0.2 } \\
\mu M)\end{array}$ & $162-0177$ & Bio-Rad \\
\hline $\begin{array}{l}\text { Clarity Max Western ECL Substrate } \\
\text { Restore Western Blot Stripping Buffer }\end{array}$ & 1705062 & Bio-Rad \\
\hline 2-mercaptoethanol & 1610710 & Thermo Fisher Scientific \\
\hline
\end{tabular}




\begin{tabular}{|l|c|c|}
\hline 30\% Acrylamide/ Bis Solution & 1610156 & Bio-Rad \\
\hline TEMED & 1610800 & Bio-Rad \\
\hline Ammonium Persulfate & 76322 & USB/Affymetrix \\
\hline Bovine Serum Albumin (BSA) & ABRE-1KG & ICP Biologicals \\
\hline
\end{tabular}

\subsubsection{Plasmids}

pX330 p53 and Pten were a gift from Tyler Jacks (Addgene plasmids \#59909 \& \#59910). Both plasmids contained loci for the respective sgRNAs, the protein coding region for Cas9 controlled by a CMV promotor, and an ampicillin resistance gene ${ }^{101}$.

PB-CMV-TO-EGFRvIII-IRES-nlsChe was a gift from Michael Elowitz (Addgene plasmid \#116039; http://n2t.net/addgene:116039; RRID: Addgene_116039). The plasmid contains the protein coding region for human EGFRvIII, followed by an IRES for mCherry and neomycin resistance, all controlled by a CMV enhancer and promotor. The plasmid also contains ampicillin resistance ${ }^{102}$.

pcDNA3-H-Ras V12 was a gift from Julian Downward (Addgene plasmid \# 39504 ; http://n2t.net/addgene:39504 ; RRID:Addgene_39504. The plasmid contains the protein coding region for H-Ras V12 controlled by a CMV enhancer and promotor, a neomycin resistance gene controlled by an SV40 promotor, and ampicillin resistance ${ }^{103}$.

pcDNA3-Flag-IDH1-R132H was a gift from Yue Xiong (Addgene plasmid \#62907; http://n2t.net/addgene:62907 ; RRID:Addgene_62907). The plasmid contains the protein coding region for human IDH1 ${ }^{\mathrm{R} 132 \mathrm{H}}$ with a conjugated Flag protein, controlled by a CMV enhancer and promotor. The plasmid also contains neomycin and ampicillin resistance genes ${ }^{104}$.

pPyCAGiPmod backbone-tdTomato was a gift from A/Prof Peter Pfeffer (VUW). 


\subsection{Cell Culture}

\subsubsection{Cell lines}

An astrocyte cell line derived from a C57BL/ 6 mouse with a stable EGFP transgene was used for this project, referred to as astrocytes. The cell line was developed by Remy Schneider and provided by Dr Melanie McConnell.

\subsubsection{Cell culture}

Astrocytes were cultured in Dulbeccos Modified Eagle Media (DMEM), with 10\% foetal bovine serum and 1\% Glutamax ${ }^{\circledR}$ (complete media). Sorted astrocytes were cultured in complete media plus 1\% penicillin-streptomycin (penstrep). Cells were grown in a T75 flask and grown to $90 \%$ confluency. All cells were incubated at $37^{\circ} \mathrm{C}$ in $5 \% \mathrm{CO}_{2}$, before passaging. When the cells reached 90\% confluent, they were washed with warmed DPBS and then covered in trypsin-EDTA. The trypsin was then aspirated, and the cells were incubated at $37^{\circ} \mathrm{C}$ for 5 minutes. Trypsin was then neutralised, and cells then resuspended in complete media at a 1:15 ratio.

\subsection{Nucleic Acid Preparation}

\subsubsection{DNA extraction}

Cells were pelleted at 400xg for four minutes. DMEM was aspirated and the pellet was washed in $5 \mathrm{~mL}$ DPBS. The cells were then resuspended in $500 \mu \mathrm{L}$ of Zymo ${ }^{\circledR}$ lysis buffer and the DNA extracted as according to the manufacturers protocol. DNA was eluted using $50 \mu \mathrm{L}$ of TE buffer, pipetted directed to the Zymo ${ }^{\circledR}$ spin colum and incubated at room temperature for 5 minutes. DNA was then transferred to a LoBind ${ }^{\circledR} 1.5 \mathrm{~mL}$ microcentrifuge tube by centrifugation at $14,100 \mathrm{xg}$ for 30 seconds, and stored at $4^{\circ} \mathrm{C}$. 


\subsubsection{DNA quantification}

DNA was quantified using the Qubit ${ }^{\circledR}$ fluorometer 2.0. A working solution of dsDNA broad range reagent was diluted in dsDNA broad range buffer at 1:200. Qubit standards were prepared using $10 \mu \mathrm{L}$ of each standard (1 and 2) was resuspended in 190 $\mu \mathrm{L}$ of working solution. $1 \mu \mathrm{L}$ of DNA sample to be quantified was resuspended in $199 \mu \mathrm{L}$ of the working solution. All samples were vortexed for 5 seconds, before incubating at room temperature for 2 minutes. The standards were read on the Qubit first to calculate a standard curve, from which the concentration of the DNA samples could be determined. Stock DNA solutions were diluted to $50 \mathrm{ng} / \mu \mathrm{L}$ as determined by the qubit calculation.

\subsubsection{RNA extraction}

Astrocytes were harvested and pelleted at 400xg for four minutes. The pellet was resuspended in $600 \mu \mathrm{L}$ of Zymo Lysis Buffer and centrifuged at 14,000xg in a Zymo SpinAway column for 30 seconds to remove cellular debris and gDNA. The lysate was then mixed 1:1 with 100\% ethanol and mixed well, before being transferred to a Zymo-Spin IIICG column and centrifuged at $14,000 \mathrm{xg}$ for 30 seconds. To further remove gDNA contamination, $400 \mu \mathrm{L}$ RNA Wash Buffer was centrifuged through the spin column and flow-through discarded. Then $5 \mu \mathrm{L}$ DNase I and $75 \mu \mathrm{L}$ DNA Digestion Buffer were incubated in the column matrix at room temperature for 15 minutes. After further washing, RNA was extracted in $100 \mu \mathrm{L}$ DNase/RNase free $\mathrm{H}_{2} \mathrm{O}$ into an RNase free microcentrifuge tube.

\subsubsection{RNA quantification}

RNA was quantified using the Qubit ${ }^{\circledR}$ fluorometer 2.0. A working solution of RNA high sensitivity reagent was diluted in RNA high sensitivity buffer at 1:200. Qubit standards were prepared using $10 \mu \mathrm{L}$ of each standard (1 and 2) was resuspended in $190 \mu \mathrm{L}$ of working solution. Then $1 \mu \mathrm{L}$ of RNA sample to be quantified was resuspended in $199 \mu \mathrm{L}$ of the working solution. All samples were vortexed for 5 seconds, before incubating at 
room temperature for 2 minutes. The standards were read on the Qubit first to calculate a standard curve, from which the concentration of the RNA samples could be determined.

\subsection{Polymerase Chain Reaction (PCR)}

\subsubsection{Primers}

\begin{tabular}{|c|c|c|}
\hline Primers & Forward & Reverse \\
\hline Pten & GCTTCTGCCATCTCTCTCCT & CGCATCCGTCTACTCCCAC \\
\hline P53 & CCATAGGGGTTTGTTTGTTTGT & CGCAGGATTTACAGACACCC \\
\hline H-Ras V12 & GGAAGCAGGTGGTCATTGAT & ACGTCATCCGAGTCCTTCA \\
\hline EGFR $v I I I$ & ATCCACGCTGTTTTGACCTC & TCCTCCATCTCATAGCTGTCG \\
\hline IDH1R132H & AAAAATCAGTGGCGGTTCTG & TATAGCTTCTGCGGCATCCT \\
\hline HPRT & \multicolumn{2}{|c|}{ Proprietary sequence designed and sourced by Qiagen. } \\
\hline
\end{tabular}

Table 2.1: Primers used in polymerase chain reaction experiments throughout the thesis.

\subsubsection{PCR}

PCR was conducted in a $20 \mu \mathrm{L}$ volume using Platinum SuperFi ${ }^{\circledR} 2 \mathrm{x}$ mastermix. Each PCR tube contained $10 \mu \mathrm{L}$ mastermix, $0.5 \mu \mathrm{M}$ of forward and reverse primers, and 50 ng of DNA. The PCR was then conducted on a Techne thermocycler, according to the following protocol (table 2.2): 


\begin{tabular}{|c|c|c|c|}
\hline \multicolumn{2}{|c|}{ Step } & Temperature & Time \\
\hline \multirow{2}{*}{ HotStart activation } & $98^{\circ} \mathrm{C}$ & 30 seconds \\
\hline \multirow{2}{*}{30 cycles } & Denature & $98^{\circ} \mathrm{C}$ & 30 seconds \\
\cline { 2 - 4 } & Anneal & $60^{\circ} \mathrm{C}$ & 30 seconds \\
\cline { 2 - 4 } & Extend & $72^{\circ} \mathrm{C}$ & 30 seconds \\
\hline \multirow{2}{*}{ Final extension } & $72^{\circ} \mathrm{C}$ & 5 minutes \\
& & $10^{\circ} \mathrm{C}$ & Hold \\
\cline { 2 - 4 } & & & \\
\hline
\end{tabular}

Table 2.2: Protocol for thermocycler used for polymerase chain reaction throughout the thesis.

\subsubsection{Reverse transcriptase PCR (RT-PCR)}

A reverse transcription reaction mix consisted of $500 \mathrm{ng}$ of $\mathrm{RNA}, 2 \mu \mathrm{L} 5 \mathrm{x}$ RT PrimeScript mastermix and made up to $10 \mu \mathrm{L}$ with $\mathrm{RNase}$ free $\mathrm{H}_{2} \mathrm{O}$. For each reaction, a negative control with no reverse transcriptase is made. The reaction was incubated in a thermocycler on the following protocol; 15 minutes at $37^{\circ} \mathrm{C}$, then 5 seconds at $80^{\circ} \mathrm{C}$, and hold at $4^{\circ} \mathrm{C}$. The RT-PCR was conducted as per 2.4.2. Assuming a complete conversion of RNA to cDNA, $50 \mathrm{ng}$ of template DNA is used.

\subsubsection{Agarose gel electrophoresis}

Electrophoresis of the PCR products was conducted on agarose gels in order to be visualised. A 1.5\% agarose-TBE solution $(w / v)$ was dissolved using microwave, before $10,000 \mathrm{x}$ SyberSafe ${ }^{\circledR}$ intercalating dye was added, and the gel was poured in a $100 \mathrm{~mL}$ cast and left to set with a lane comb. PCR samples were resuspended with Orange $G^{\circledR}$ loading dye at 6:1 and pipetted into separate lanes. The electrophoresis was run using Trackit ${ }^{\circledR} 100$ 
bp ladder at $120 \mathrm{~V}$ for 1 hour (or until satisfactory separation of the dye front was seen). Gels were visualised using fluorescence stimulated with a $532 \mathrm{~nm}$ laser, using the Typhoon ${ }^{\circledR}$ scanner.

\subsection{Sequencing}

\subsubsection{PCR product clean and concentrate}

PCR products were mixed in DNA binding buffer at 1:5 and prepared for sequencing according to the $\mathrm{Zymo}^{\circledR}$ clean and concentrate protocol. The eluate was centrifuged at 13,000xg for 30 seconds and collected in a LoBind ${ }^{\circledR} 1.5 \mathrm{~mL}$ microcentrifuge tube. The PCR products were then quantified using the Qubit ${ }^{\circledR}$

\subsubsection{Preparing PCR samples for sequencing}

Samples were prepared for sequencing according to the Massey Genome Services guidelines. For products larger than 200 bp $12.5 \mathrm{ng}$ of PCR product was mixed with 4 pmol of primer, and the volume made up to $20 \mu \mathrm{L}$. Separate samples were prepared separately using forward and reverse primers. The samples were then sent to Massey Genome Services, Palmerston North, to be sequenced using Sanger Sequencing.

\subsubsection{Oxford Nanopore sequencing}

Following a PCR clean and concentrate, $400 \mathrm{fmol}$ of DNA was diluted to $48 \mu \mathrm{L}$ of DNase-free $\mathrm{H}_{2} \mathrm{O}$. An end trimming reaction mix was then added to a PCR tube; consisting of the $48 \mu \mathrm{L}$ PCR product, $3.5 \mu \mathrm{L}$ NEBNext FFPE DNA repair buffer, $2 \mu \mathrm{L}$ NEBBext FFPE DNA repair mix, $3.5 \mu \mathrm{L}$ Ultra II End-prep reaction buffer, $3 \mu \mathrm{L}$ Ultra II End-prep enzyme mix. In a thermocycler, this reaction was incubated at $20{ }^{\circ} \mathrm{C}$ for 5 minutes. The DNA samples were then resuspended with $60 \mu \mathrm{L}$ of AMPure XP beads in a $1.5 \mathrm{~mL}$ microcentrifuge tube. To remove fragmented DNA, this solution was rotated for 5 minutes 
and then washed while on a magnetic rack with $200 \mu \mathrm{L} 70 \%$ ethanol to remove trimmed DNA, the supernatant was carefully removed, and the wash process repeated. The DNA was then eluted from the beads using $25 \mu \mathrm{L}$ of DNase-free $\mathrm{H}_{2} \mathrm{O}$ and quantified using a Qubit.

Following end-prepping, $200 \mu \mathrm{L}$ of DNA was diluted to $22.5 \mu \mathrm{L}$ of DNase-free $\mathrm{H}_{2} \mathrm{O}$. This solution was then mixed with $2.5 \mu \mathrm{L}$ of a unique Native barcode and $25 \mu \mathrm{L}$ of Blunt/TA Ligase master mix. This barcoding reaction was incubated at room temperature for 10 minutes. As previous, using $50 \mu \mathrm{L}$ of AMPure XP beads were mixed with the barcoded DNA in a $1.5 \mathrm{~mL}$ microcentrifuge tube. The solution was rotated for 5 minutes and then washed while on a magnetic rack with $200 \mu \mathrm{L} 70 \%$ ethanol to remove unligated barcodes, the supernatant was carefully removed, and the wash process repeated. The barcoded DNA was then eluted in $26 \mu \mathrm{L}$ DNase-free $\mathrm{H}_{2} \mathrm{O}$. Following quantification using a Qubit, up to $200 \mathrm{fmol}$ of each sample to be sequenced were then pooled into a single 1.5 $\mathrm{mL}$ microcentrifuge tube.

The pooled, barcoded DNA were then diluted to $65 \mu \mathrm{L}$ of DNase-free $\mathrm{H}_{2} \mathrm{O}$, along with $5 \mu \mathrm{L}$ Adapter Mix II, $20 \mu \mathrm{L}$ NEBNext Quick Ligation Reaction mix, $10 \mu \mathrm{L}$ Quick T4 DNA Ligase. This reaction incubated at room temperature for 10 minutes. As previous, using $50 \mu \mathrm{L}$ of AMPure XP beads were mixed with the barcoded DNA in a $1.5 \mathrm{~mL}$ microcentrifuge tube. The solution was rotated for 5 minutes and then washed while on a magnetic rack with $250 \mu \mathrm{L}$ Short Fragment Buffer, the supernatant was carefully removed, and the wash process repeated. The DNA was eluted in $15 \mu \mathrm{L}$ oxford nanopore elution buffer.

\subsection{Plasmid Preparation}

\subsubsection{Bacterial preparation}

Transformed E. coli sourced from Addgene (Watertown, Massachusetts, USA) were received as bacterial stabs. LB-Agar plates with $50 \mathrm{ng} / \mathrm{mL}$ ampicillin were used to isolate 
single colonies of transformed bacteria. Single colonies were inoculated into $5 \mathrm{~mL} \mathrm{LB}$ broth with $50 \mathrm{ng} / \mathrm{mL}$ ampicillin and incubated at $37^{\circ} \mathrm{C}$ with $8 \% \mathrm{CO}_{2}$ in a shaking incubator for 6 hours. The liquid culture was then transferred to $200 \mathrm{~mL} \mathrm{LB}$ broth with ampicillin and incubated overnight.

\subsubsection{Plasmid isolation}

Overnight cultures were transferred to $500 \mathrm{~mL}$ centrifuge bottles and centrifuged at $4000 \mathrm{xg}$ for 10 minutes at $4^{\circ} \mathrm{C}$. The supernatant was discarded, and the bacterial pellet resuspended in $12 \mathrm{~mL}$ Macherey-Nagel resuspension buffer and the plasmids were extracted according to the manufacturers protocol. Plasmids were eluted in $15 \mathrm{~mL}$ Macherey-Nagel elution buffer into a $50 \mathrm{~mL}$ centrifuge tube. The plasmid was precipitated in $10.5 \mathrm{~mL}$ isopropanol and incubated at room temperature for five minutes. The precipitated solution was then transferred to a $50 \mathrm{~mL}$ glass centrifuge and centrifuged at $20,000 \mathrm{xg}$ for 15 minutes at $4^{\circ} \mathrm{C}$. The supernatents were discarded carefully and the pelleted plasmid DNA was washed with $70 \%$ ethanol by brief centrifugation. Plasmid DNA was eluted in $1 \mathrm{~mL}$ TE buffer and quantified using Qubit fluorometry.

\subsection{Transfection}

\subsubsection{Transfection using lipofection}

Astrocytes were plated at 25-30\% confluency in a 6-well plate in complete DMEM and incubated overnight at $37^{\circ} \mathrm{C}$ with $5 \% \mathrm{CO}_{2}$. The following morning, when wells reach $50-60 \%$ confluency, the astrocytes have the media replaced with DMEM supplemented with Glutamax ${ }^{\circledR}$ and no FBS. The astrocytes were starved of FBS for four hours while incubated at $37^{\circ} \mathrm{C}$ at $5 \% \mathrm{CO}_{2}$. Viafect ${ }^{\circledR}$ transfection reagent was used to transfect the CRISPR plasmids into the astrocytes. A transfection complex of $500 \mathrm{ng}$ tdTomato : 500 ng CRISPR plasmid : $3 \mu \mathrm{L}$ Viafect reagent : $500 \mu \mathrm{L}$ FBS-free DMEM per well was incubated at room temperature for 20 minutes. $500 \mu \mathrm{L}$ of transfection complex was 
pipetted into each well and the cells are incubated for a further 5 hours. FBS-free DMEM is aspirated from each well and replaced with $2 \mathrm{~mL}$ prewarmed complete DMEM.

\subsubsection{Fluorescence activated cell sorting (FACS) of transfected cells}

Transfected cells were incubated at $37^{\circ} \mathrm{C}$ with $5 \% \mathrm{CO}_{2}$ for 48 hours. Periodically, the astrocytes were observed for tdTomato expression using fluorescent microscopy. After 48 hours, the cells were washed with PBS and lifted (as per 2.2.2) into a $15 \mathrm{~mL}$ centrifuge tube. The cells were centrifuged at 400xg for 5 minutes and the media aspirated. The pellet was then washed with cold PBS and centrifuged at 400xg for a further 5 minutes, twice. The cells were then resuspended at $4 \times 10^{6}$ cells $/ \mathrm{mL}$ in cold FACS buffer (PBS, $0.5 \%$ BSA or 5\% FBS, $0.1 \% \mathrm{NaN} 3$ ) and pipetted into a polyethylene FACS tube through a $70 \mu \mathrm{m}$ filter. The samples were transported on ice to the Malaghan Institute of Medical Research for FACS using the BD Influx ${ }^{\mathrm{TM}}$ Cell Sorter. Cells were gated on a) singlets, b) viability through DAPI exclusion, and c) $\mathrm{EGFP}^{+}$and tdTomato $^{+}$double positives astrocytes.

\subsubsection{Culturing of single cell populations}

Conditioned DMEM media was removed from 50\% confluent astrocytes. This media was then filtered using a $50 \mathrm{~mL}$ syringe and a $22 \mu \mathrm{m}$ pore filter. In a 96 -well plate, each well was aliquoted $100 \mu \mathrm{L}$ supplemented DMEM with penicillin-streptomycin (penstrep) and $100 \mu \mathrm{L}$ conditioned DMEM. Single cells were index sorted in the 96-well plate (2.7.2) and incubated at $37^{\circ} \mathrm{C}$ with $5 \% \mathrm{CO}_{2}$ for two weeks. Each well was individually assessed for a) individual colonies to ensure only a single cell had been sorted, and b) the colony size including the presence of senescent cells by eye. The colonies which had a significant growth over the two weeks and had minimal senescence were lifted and passaged into a 24-well plate. This process was continued, with the growth of the clonal colonies observed daily. The colonies which continued to show growth were passaged through 12-well plates, 6-well plates, T25 flasks and finally T75 flasks. From this point the clonal cells were assessed through the heteroduplex assay (2.8) and western blot (2.9). 


\subsubsection{Culturing of bulk sorted populations}

Following the single cell index sort, the remaining cells gated by 2.7.2 were sorted into $5 \mathrm{~mL}$ of supplemented DMEM with penstrep in a $15 \mathrm{~mL}$ centrifuge tube. These cells were then transferred to a single well of a 6-well plate and left to adhere in an incubator at $37^{\circ} \mathrm{C}$ with $5 \% \mathrm{CO}_{2}$ for two days. Once the well reached $80 \%$ confluence, the cells were passaged into a T25 flask. Once confluence is reached these cells were assessed through the heteroduplex assay (2.8) and western blot (2.9).

\subsubsection{G418 drug titration}

Astrocytes were plated at 25\% confluence in a 48 -well plate and incubated over night at $37^{\circ} \mathrm{C}$ with $5 \% \mathrm{CO}_{2}$ overnight. The cells were treated with $\mathrm{G} 418$ with increasing concentrations from $0 \mu \mathrm{g} / \mathrm{mL}$ to $630 \mu \mathrm{g} / \mathrm{mL}$. Wells were visually assessed for cell viability every 24 hours for three days. The astrocytes were pelleted and washed three times in cold PBS, then each well was resuspended in $100 \mu \mathrm{L}$ of cold FACS buffer. The cells were processed by flow cytometry for 30 seconds at a medium flow rate

\subsubsection{Stable transfection}

Six-well plates were set up at $25 \%$ confluence and incubated overnight at $37^{\circ} \mathrm{C}$ with 5\% $\mathrm{CO}_{2}$ overnight. Transfection protocol was conducted as per 2.7.1. For every two wells which were transfected with the plasmids to be stably introduced, one well was transfected with tdTomato as a negative control for selection.

After 48 hours, G418 was introduced to the wells. The astrocytes were monitored for a further 24 hours for signs of cell death. The wells were then washed, lifted and left to adhere with new G418-DMEM. Once the astrocytes reached 80\% confluence, the wells were passaged into a T25 flask. Every 2-3 days, the G418-DMEM was refreshed. After 23 weeks, the cells were harvested and RT-PCR (2.4.3) was conducted to confirm the stable transfection of the introduced plasmids. 


\subsection{Heteroduplex Assay}

\subsubsection{Reannealing PCR products}

PCR products of CRISPR targeted astrocytes were isolated using Zymo ${ }^{\circledR}$ clean and concentrate kit. Concentrated PCR products were diluted in a solution of $10 \mu \mathrm{M} \mathrm{MgCl}$, $10 \mu \mathrm{M}$ Tris- $\mathrm{Cl}$ and $50 \mu \mathrm{M} \mathrm{NaCl}$ to a total volume of $30 \mu \mathrm{L}$ in a PCR tube. PCR products were then denatured at $95^{\circ} \mathrm{C}$ for 5 minutes and slowly reannealed with temperature decreasing at $1{ }^{\circ} \mathrm{C} /$ second down to $25^{\circ} \mathrm{C}$.

\subsubsection{Surveyor assay}

Reannealed PCR products were transferred into new $200 \mu \mathrm{L}$ microcentrifuge tube with $15 \mu \mathrm{L}$ in each and rested on ice. The endonuclease solution was made of $15 \mu \mathrm{L}$ reannealed PCR product, $1 \mu \mathrm{L}$ Surveyor ${ }^{\circledR}$ nuclease, $1 \mu \mathrm{L}$ Surveyor ${ }^{\circledR}$ enhancer and $1.7 \mu \mathrm{L}$ $150 \mu \mathrm{M} \mathrm{MgCl}_{2}$. The digest reaction was incubated in a $42^{\circ} \mathrm{C}$ water bath for 60 minutes, and the reaction terminated with the stop solution $(10 \% v / v)$.

\subsubsection{Nondenaturing acrylamide gel electrophoresis}

Digested products (cut) and non-digested products (uncut) were visualised on a 15\% nondenaturing acrylamide gel. The acrylamide gel was made from $5 \mathrm{~mL} \mathrm{30 \%}$ acrylamide (29:1), $3 \mathrm{~mL} \mathrm{ddH}{ }_{2} \mathrm{O}, 2 \mathrm{~mL} 5 \times$ TBE, $200 \mu \mathrm{L} 10 \% \mathrm{APS}(w / v)$, and $10 \mu \mathrm{L}$ TEMED. The gel was loaded in a tank with 1x TBE running buffer, and pre-run for 10 minutes at $100 \mathrm{~V}$. Products were mixed with Orange $G^{\circledR}$ loading dye 5:1 and run in the acrylamide gel for 2.5 hours at $100 \mathrm{~V}$, with constant voltage at room temperature.

DNA products were post-stained with SybrSafe ${ }^{\circledR}$. In a lightproof container, $50 \mathrm{~mL}$ 1x TBE and $15 \mu \mathrm{L}$ SybrSafe ${ }^{\circledR}$ were combined and the acrylamide gel was rocked at room temperature for 30 minutes. Gels were visualised using fluorescence stimulated with a 532 nm laser, with the Typhoon ${ }^{\circledR}$ scanner. 


\subsection{Western Blot}

\subsubsection{Buffers}

SDS Running Buffer; $14.4 \mathrm{~g}$ Glycine, $3 \mathrm{~g}$ Tris, $1 \mathrm{~g}$ SDS, made to $1 \mathrm{~L}$ with $\mathrm{ddH}_{2} \mathrm{O}$.

Transfer Buffer; $14.4 \mathrm{~g}$ Glycine, $3 \mathrm{~g}$ Tris, $200 \mathrm{~mL}$ Methanol, made up to $1 \mathrm{~L}$ with dd $\mathrm{H}_{2} \mathrm{O}$.

Crude Lysis Buffer; $70 \mathrm{mM} \mathrm{NaCl}, 20 \mathrm{mM}$ Tris, $0.1 \%$ IGEPAL $(v / v)$.

Laemmli Buffer; $20 \%$ glycerol $(v / v), 15$ mg/mL Tris-Cl (pH 6.8), 0.1\% Bromophenol blue $(w / v), 20 \mathrm{mM}$ dithiothreitol, $40 \mathrm{mg} / \mathrm{mL}$ SDS.

Ponceau Red; $0.2 \%$ Ponceau S $(w / v), 3 \%$ acetic acid $(v / v)$.

PBS-T; 2x Thermo Fisher PBS Tablets dissolved in $1 \mathrm{~L} d d \mathrm{~d}_{2} \mathrm{O}, 0.05 \%$ Tween-20 $(v / v)$.

\subsubsection{Gel composition}

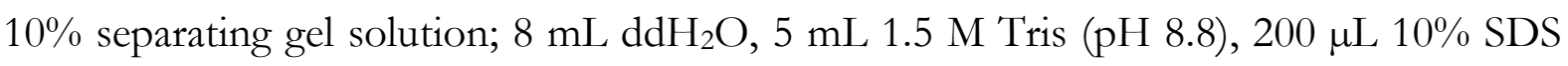
$(w / v), 6.66 \mathrm{~mL}$ Acrylamide (29:1), $100 \mu \mathrm{L}$ 10\% APS $(w / v), 10 \mu \mathrm{L}$ TEMED.

4\% stacking gel solution; $6.1 \mathrm{~mL} \mathrm{ddH}_{2} \mathrm{O}, 2.55 \mathrm{~mL} 0.5 \mathrm{M}$ Tris (pH 6.8), $100 \mu \mathrm{L} 10 \% \mathrm{SDS}$ $w / v), 1.33 \mathrm{~mL}$ Acrylamide (29:1), $50 \mu \mathrm{L}$ APS $(w / v), 10 \mu \mathrm{L}$ TEMED. 


\subsubsection{Antibodies}

\begin{tabular}{|c|c|c|}
\hline Antibody & Catalogue No. & Company \\
\hline pAkt Ser473 & 9271 & Cell Signalling \\
\hline PTEN & 95595 & Cell Signalling \\
\hline P53 & PAb240 AbCam \\
\hline B Actin & 5441 & Sigma \\
\hline Goat anti-Rabbit & 150166 & AbCam \\
\hline Goat anti-Mouse & 405306 & Biolegend \\
\hline
\end{tabular}

Table 2.3: Antibodies used throughout the thesis.

\subsubsection{Serum starvation and BEZ235 treatment}

The astrocytes were plated in a T75 flask at 50\% confluency in complete DMEM and incubated overnight at $37^{\circ} \mathrm{C}$ in $5 \% \mathrm{CO}_{2}$ to adhere. Following this, the flasks had their media replaced with serum-free DMEM (with Glutamax ${ }^{\circledR}$ ) and incubated at $37^{\circ} \mathrm{C}$ in $5 \%$ $\mathrm{CO}_{2}$ for a further 18 hours to starve the astrocytes. One hour prior to replacing media, 7.5 $\mu \mathrm{M}$ BEZ235 was added to appropriate flasks to inhibit PI3K and mTOR. The media was then replaced again with prewarmed complete DMEM, without BEZ235, and incubated at $37^{\circ} \mathrm{C}$ in $5 \% \mathrm{CO}_{2}$ for 15 minutes to stimulate. The astrocytes were washed with PBS and the supernatant discarded, then the cells were harvested.

\subsubsection{Irradiation of cell lines}

T75 flasks were transferred to MIMR. Cells were given 2 Gy of radiation and then incubated at $37^{\circ} \mathrm{C}$ with $5 \% \mathrm{CO}_{2}$ for 2 hours. Irradiated cells and negative controls were harvested in cold PBS and centrifuged at 400xg for 4 minutes. 


\subsubsection{Protein extraction}

The cell pellet was resuspended in $70 \mu \mathrm{L}$ crude lysis buffer along with $0.7 \mu \mathrm{L}$ phosphatase and protease inhibitor. The solution was then resuspended approximately 20 times and incubated on ice for 60 minutes. The lysate was centrifuged at $20,000 \mathrm{xg}$ at $4^{\circ} \mathrm{C}$ for 5 minutes. The supernatant was carefully pipetted into a new microcentrifuge tube and the pellet discarded.

\subsubsection{Protein quantification and preparation}

Protein was quantified using the Qubit ${ }^{\circledR}$ fluorometer 2.0. A working solution of Qubit protein reagent was diluted in Qubit protein buffer at 1:200. Qubit standards were prepared using $10 \mu \mathrm{L}$ of each standard (1, 2 and 3) was resuspended in $190 \mu \mathrm{L}$ of working solution. $1 \mu \mathrm{L}$ of protein-lysate supernatant sample to be quantified was resuspended in $199 \mu \mathrm{L}$ of the working solution. All samples were vortexed for 5 seconds, before incubating at room temperature for 15 minutes. The standards were read on the Qubit first to calculate a standard curve, from which the concentration of the protein samples could be determined. The protein samples were then prepared to be run on an SDS-Polyacrylamide Gel. An aliquot of $50 \mu \mathrm{g}$ of protein lysate was resuspended in 3:1 in Laemmli Buffer and boiled at $95^{\circ} \mathrm{C}$ for 5 minutes.

\subsubsection{SDS-polyacrylamide gel preparation}

Plates for $1.5 \mathrm{~mm} \mathrm{BioRad}{ }^{\circledR}$ gel cassettes were thoroughly washed with isopropanol and a Kimwipe ${ }^{\circledR}$ and rinsed with 100\% ethanol, then left to dry. The plates were then loaded in a cassette holder and the bottom sealed with Parafilm ${ }^{\circledR}$, then placed in a casting rack. In a centrifuge tube, the separating gel was prepared (2.9.2) and pipetted into the cassette with enough room for the stacking gel. Isopropanol was poured on top to remove bubbles, and the gel was left to dry at room temperature for 60 minutes. The isopropanol 
was poured off, and in another centrifuge tube, the stacking gel was prepared (2.9.2). The stacking gel was then loaded to the top of the separating gel, then an appropriately sized $1.5 \mathrm{~mm}$ lane comb added to set at room temperature for 60 minutes.

\subsubsection{SDS-polyacrylamide gel electrophoresis (SDS-PAGE)}

SDS-Polyacrylamide gel was loaded into a gel tank with the lanes facing inwards and the tank filled with cold 1x Running buffer. If only one gel was used per run, then a clean empty cassette was inserted to ensure an even seal. The lane comb was carefully removed and, for each sample, $50 \mu \mathrm{g}$ of denatured protein in Laemmli buffer (2.9.5) was aliquoted. With a lane separating it from the samples, $3 \mu \mathrm{L}$ of PageRuler Plus Prestained Protein Ladder was pipetted. The gel was run at $170 \mathrm{~V}$ with a constant voltage for one hour, or until satisfactory migration of the dye front was seen.

\subsubsection{Western blot}

After the SDS-PAGE has been completed, a piece of PVDF membrane was cut to a size appropriate to the number of lanes used and activated by exposure to $100 \%$ methanol. The transfer cassette was then prepared. In a transfer tray, cold Transfer buffer was poured into both compartments, and sponge placed in the smaller side to soak. In the larger side, an open transfer cassette was placed black side down with a second sponge placed on top. Two pieces of filter paper were cut to be larger than the membrane and each was placed separately on a sponge. The SDS-Polyacrylamide gel was removed from the cassette, and if needed it was cut to the size of the lanes used. The gel was then placed on top of the filter paper in the cassette stack. Carefully using tweezers, the PVDF membrane was moved from the methanol to be placed on top of the gel. A roller was used to ensure there were no bubbles between the gel and the membrane. After this, the second piece of filter paper was then placed on the membrane followed by the second sponge, then the cassette was closed. The cassette was then placed in a gel tank along with an ice pack. If only one SDS-polyacrylamide gel was being transferred, an empty cassette was 
used to ensure a tight seal. The tank was then filled with cold Transfer buffer. The transfer was conducted at $300 \mathrm{~mA}$, with a constant amperage at room temperature, for two hours.

The membrane was then rocked for three minutes in Ponceau Red stain and then in PBS to ensure protein was transferred to the membrane. On a clean glass cassette, the membrane was then trimmed to where the protein was visible. In a 5\% solution of BSA in PBS $(w / v)$, the membrane was blocked on a rocker for 60 minutes at room temperature. The primary detection antibodies were then diluted 1:1000 in $5 \mathrm{~mL} \mathrm{5 \%} \mathrm{BSA} \mathrm{and} \mathrm{poured}$ on the membrane to rock overnight at $4^{\circ} \mathrm{C}$. The membrane was washed three times with PBS-T for five minutes each. The secondary antibodies were diluted 1:7000 in $7 \mathrm{~mL}$ PBS$\mathrm{T}$ and rocked for 60 minutes at room temperature. The membrane was again washed three times with PBS-T for five minutes each. In a microcentrifuge tube, $500 \mu \mathrm{L}$ of Clarity Max Western Peroxide Reagent was mixed with $500 \mu \mathrm{L}$ Clarity Max Western Luminol/Enhancer Reagent and then pipetted onto a clean glass slide. The membrane was briefly immersed in the ECL substrate and placed flat in a plastic sheet which is folded to cover both sides. The membrane was rolled to ensure that the substrate is evenly spread and that there was no excess. Proteins were visualised using a CCD camera (Amersham Imager 600, GE Healthcare Life Sciences). After the blot has been visualised, the antibodies were then stripped using Restore Western Blot Stripping Buffer and incubated for 15 minutes and washed briefly in PBS-T for five minutes. The membrane was then blocked again with 5\% BSA and the process repeated to investigate the different proteins.

\subsection{Cancer Cell Assays}

\subsubsection{Clonogenic assay}

Astrocytes were lifted and suspended in $10 \mathrm{~mL}$ DMEM then counted using a haemocytometer. Solutions were then prepared at $300 \mathrm{cell} / \mathrm{mL}$ and then aliquoted into a 6 -well plate with 15, 30 and 60 cells per well. The 6 -well plates were incubated at $37^{\circ} \mathrm{C}$ with $5 \% \mathrm{CO}_{2}$ for two weeks. 
The media was then aspirated, and the wells washed with cold PBS and rocked for five minutes. The cells were fixed and permeabilised with $1 \mathrm{~mL} 6 \%$ neutral buffered formalin (NBF) at room temperature for 30 minutes while being rocked. After the NBF was disposed of, $1 \mathrm{~mL}$ of methylene blue was aliquoted into each well and rocked at room temperature for two hours, until the colonies were visible and vivid to the eye. The methylene blue was removed, then using a sink of tap water the plates were then washed three times, careful not to agitate the colonies. The plates were then left to dry on the bench top overnight. Using an Epson scanner, the plates were then scanned and analysed using ImageJ. The colonies were visualised using the threshold filter. The area of the well that the colonies occupied was then compared with the total area of the well. The area of the well which the colonies occupied was then compared with the total area of the well; this represented the relative area which the colonies occupied.

\subsubsection{Growth curve analysis on Incucyte}

Astrocytes were lifted and suspended in $10 \mathrm{~mL}$ DMEM then counted using a haemocytometer. Aliquot 1000 cell/well into a 6-well plate, in triplicate per cell line, to a total of $2 \mathrm{~mL}$ per well. Incubate cells at $37^{\circ} \mathrm{C}$ with $5 \% \mathrm{CO}_{2}$ for 2 hours for cells to adhere to the plate. The plates are then transferred to MIMR and placed in the Incucyte ${ }^{\circledR}$. The IC software was set to capture 36 pictures per well every four hours, over a five-day period. The Incucyte ${ }^{\circledR}$ was checked daily to ensure a) consistent pictures and b) the media is not evaporating due to low incubator water levels.

\subsubsection{Wound healing assay on Incucyte}

A T25 flask of astrocytes were incubated until 80\% confluence, then lifted in $10 \mathrm{~mL}$ DMEM. In a 96-well plate, $100 \mu \mathrm{L}$ of suspended astrocytes are aliquoted into triplicate columns, and $100 \mu \mathrm{L}$ of media added to a total of $200 \mu \mathrm{L}$. The 96 -well plate is then incubated overnight at $37^{\circ} \mathrm{C}$ with $5 \% \mathrm{CO}_{2}$ to allow the wells to grow to $100 \%$ confluence. The following day, the plates are transferred to MIMR. Using a sterilised Incucyte ${ }^{\circledR}$ wound maker 96, a uniform wound is made to all wells of the plate and the plate is then washed 
with PBS. Each well had $200 \mu \mathrm{L}$ warmed DMEM added and placed in the Incucyte ${ }^{\circledR}$. The IC software was set to capture a single image of each well every hour for 12 hours.

\subsubsection{MTS Metabolic Assay}

An 80\% confluent T75 flask of astrocytes were lifted in $10 \mathrm{~mL}$ DMEM and counted using a haemocytometer. The cell suspensions were diluted to $10^{4}$ cell $/ \mathrm{mL}$, and $100 \mu \mathrm{L}$ per well were pipetted into a 96-well plate. Plates were set up excluding the outer most wells to minimise evaporation, technical replicates of each cell line well pipetted per column and the final column consisting of a media only control. Four plates were established and labelled day 1 through 4 and incubated at $37^{\circ} \mathrm{C}$ with $5 \% \mathrm{CO}_{2}$. After 24, 48, 72, and 96 hours, the respective plates were removed and $20 \mu \mathrm{L}$ of CellTitre 96 AQueous $^{\mathrm{TM}}$ was pipetted into each well, the plate then incubated for a further 45 minutes. The absorbance of the plate was then read at $490 \mathrm{~nm}$.

\subsubsection{Cell cycle assay}

Astrocytes were plated at 25\% confluence in a T25 flask and incubated overnight at $37^{\circ} \mathrm{C}$ with $5 \% \mathrm{CO}_{2}$. The flasks were transported to MIMR and irradiated with $6 \mathrm{~Gy}$. After a further 8 hours of incubation, the cells were then lifted and diluted to $10^{6} \mathrm{cell} / \mathrm{mL}$ in PBS. Then $300 \mu \mathrm{L}$ of the cell suspension was pipetted drop wise into $700 \mu \mathrm{L}$ of ice cold $100 \%$ ethanol, which was vortexed continuously throughout to ensure no cell clumping. These fixed and permeabilised cells were then centrifuged at $3000 \mathrm{rpm}$ for 5 minutes, and the supernatant discarded. The pelleted cells were then resuspended in $400 \mu \mathrm{L}$ of a DNA staining solution $(0.1 \mathrm{mg} / \mathrm{mL}$ PI and $0.25 \mathrm{mg} / \mathrm{mL}$ RNaseA in PBS). The cell suspensions were filtered into polypropylene FACS tubes through a $0.75 \mu \mathrm{m}$ filter. Using the BD FACSCanto II, flow cytometry was conducted using a medium flow rate, until 20,000 events had been recorded. 


\subsection{Statistical Analysis}

Graphing and statistical analyses were conducted using Prism v8.0 software. Analyses included one-way ANOVA, unpaired $t$-tests and area under curve (AUC) calculations. Significance for these tests was set at 5\%. Tukey's post-hoc test was used when appropriate with multiple comparisons correction. 


\subsection{Development and Screening of}

\section{CRISPR/Cas9 Induced Knockouts of Pten}

\section{and P53}

\subsection{Introduction}

The first stage of this project was to develop two EGFP knockout astrocyte cell lines; Pten and P53. This was achieved through transfection of a plasmid via the lipofection method. Because the CRISPR/Cas9 mediated mutations are not 100\% efficient, a comprehensive series of screening assays were developed and optimised in order to ensure that the intended knockouts had been developed. These assays included the heteroduplex assay to screen for indels, MinION sequencing of the loci, and western blot to investigate the expressional effects of these mutations.

\subsection{Confirmation of sgRNA binding site sequence}

\subsection{1 pX330 CRISPR plasmids}

In order to induce the indel mutations in the Pten and P53 genes, the plasmids pX330 Pten and pX330 P53 were purchased ${ }^{96}$. These plasmids were used to induce Pten and P53 knockouts in mouse hepatocytes in vivo; they contained the sequence for both the Cas9 protein and the sgRNA specific to their respective target gene (Fig 3.1). 


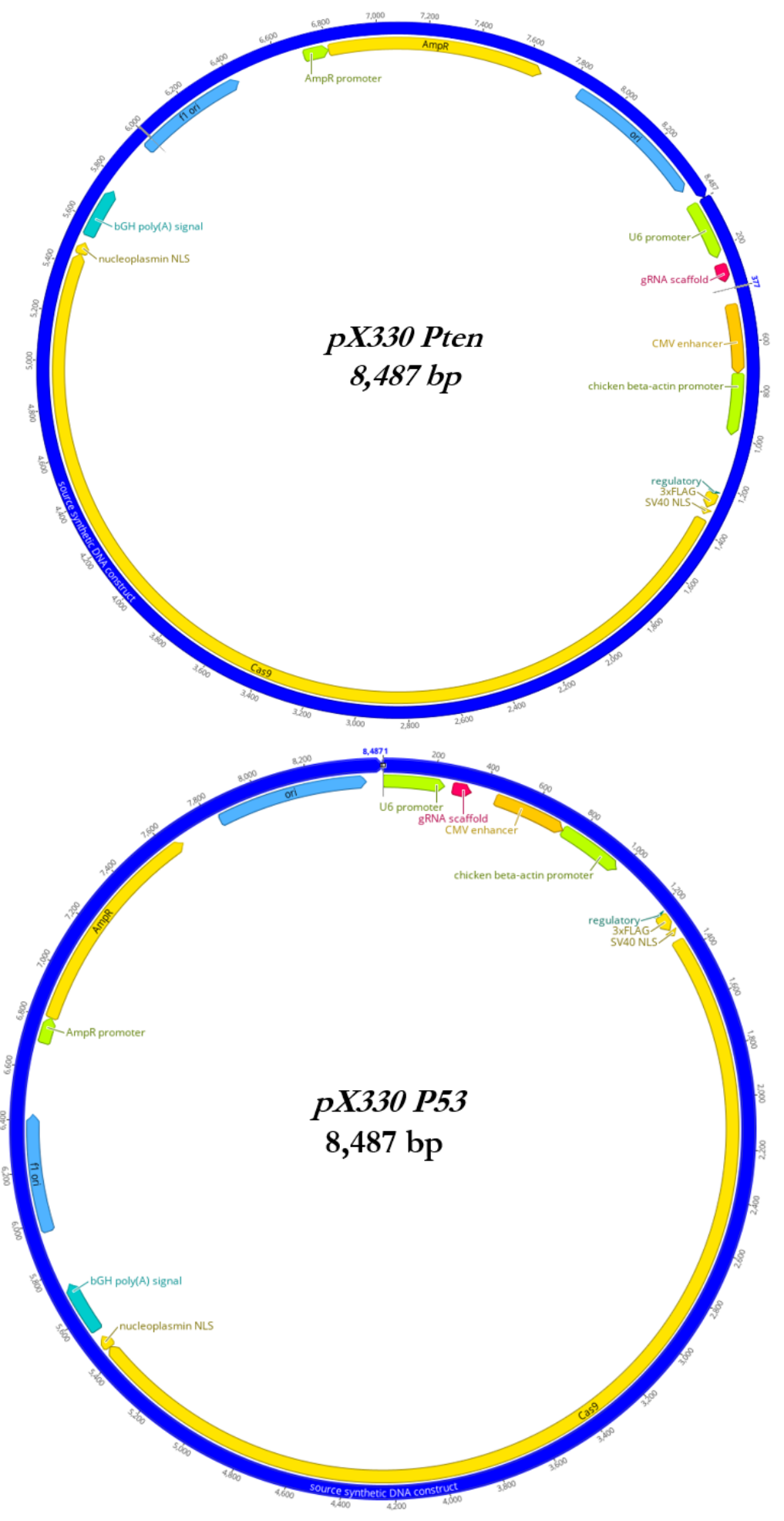

Figure 3.1: pX330 Pten and P53 plasmids. Both plasmids contain coding sequences for Cas9 (yellow), ampicillin resistance (orange), and a specific sgRNA binding site and scaffold sequence (pink). The promotors for these coding sequences are shown in green. 


\subsubsection{Confirmation of sgRNA binding site}

Before using the plasmids, it was necessary to confirm the genomic DNA sequence at each locus in the astrocyte cell line to ensure the $\operatorname{sgRNA}$ would recognise and direct the Cas9 to cut the correct site. PCR primers were designed to flank the sgRNA binding site for each of the pX330 Pten and P53 plasmids. Using gDNA extracted from the astrocytes, an endpoint PCR was conducted for both Pten and P53 loci. The PCR products were run on an 1.5\% TBE-agarose gel to confirm the correct sized band (Fig 3.2A and B). These products were then prepared for Sanger sequencing to confirm that the sequences of these PCR products were complementary to the pX330 sgRNA and contained an appropriate PAM sequence (Fig 3.2C, D, E and F). This result confirmed that the astrocytes had the complementary sequence for both the pX330 Pten and P53 sgRNA, and that these plasmids were appropriate for use in this investigation. 

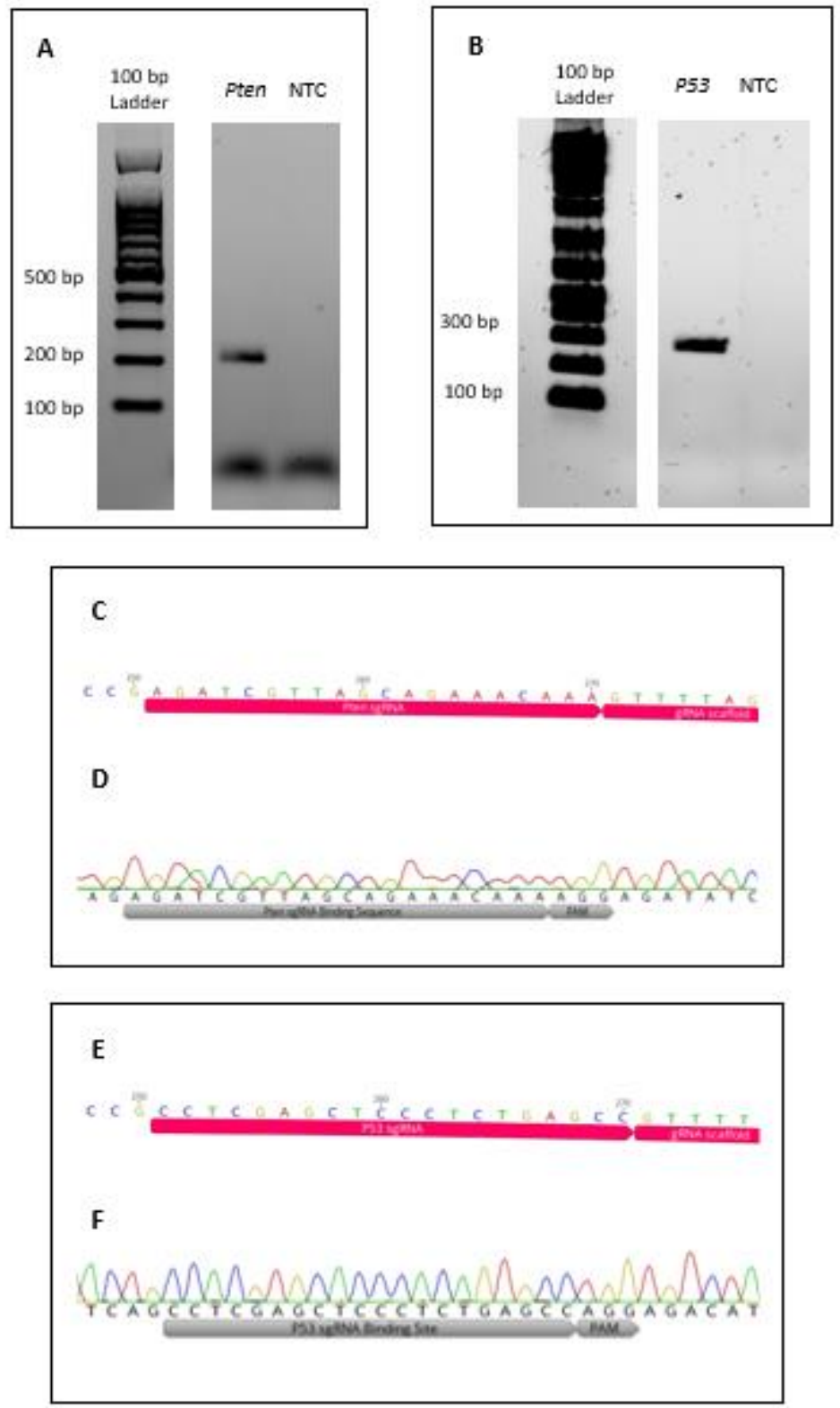

Figure 3.2: PCR amplification of sgRNA binding sites. A \& B. Pten and P53 PCR products from astrocytes gDNA. C. Pten sgRNA sequence on pX330 Pten plasmid and D. Sanger sequencing results for astrocytes Pten PCR product. E. P53 sgRNA sequence on pX330 P53 plasmid and F. Sanger sequencing results for astrocytes P53 PCR product. 


\subsection{Development of Pten and P53 knockout mutations}

\subsubsection{Co-transfection of pX330 and tdTomato plasmids}

In order to screen for successful transfections, a plasmid for a red fluorescent marker, tdTomato, was co-transfected alongside the pX330 plasmids. This allowed for the results of the transfection to be visible within 24 hours, and for fluorescence activated cell sorting (FACS) to be used to enrich the successfully transfected populations as the pX330 plasmid itself did not contain any selective or fluorescent markers. Both single cell populations and bulk populations of transfected cells were isolated (Fig 3.3).

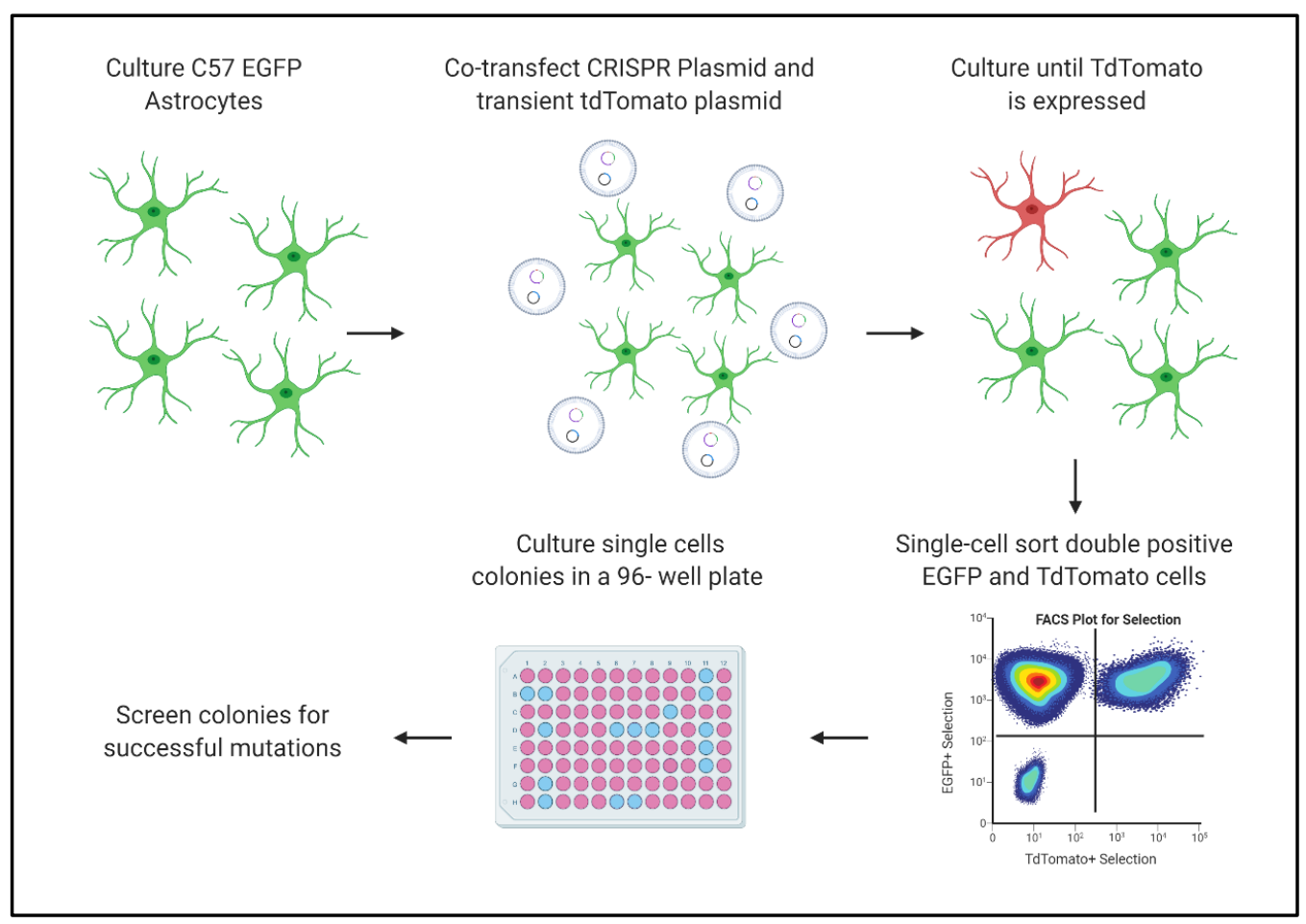

Figure 3.3: Strategy for developing CRISPR knockout clonal populations.

Created in BioRender. 
At 24 hours following the introduction of the plasmid, fluorescent microscopy confirmed that the tdTomato had successfully been transfected into the astrocytes, and the assumption was made that the pX330 plasmid was likely to be expressed as well. This is because when two plasmids are co-transfected using lipofection, virtually $100 \%$ of transfected cells show expression of both plasmids 105 .

Forty-eight hours after transfection, the astrocytes were sorted using the FACS Influx, which also yielded an accurate transfection efficiency. The Pten pX330 population contained 1.7\% EGFP-TdTomato double-positive cells, while the P53 pX330 population contained 29.9\% (Fig 3.4C and D). Following this, a gating strategy was developed. Double-positive, single cells of a moderate size were sorted, with DAPI exclusion as a viability indicator (Fig $3.4 \mathrm{E}$ and F). Two samples were sorted using FACS. Single cell 'clonal' populations were first sorted into individual wells of a 96-well plate, using an index sort to ensure only one transfected cell was transferred to each well. The remaining transfected cells were 'bulk' sorted into a $15 \mathrm{~mL}$ centrifuge tube. 


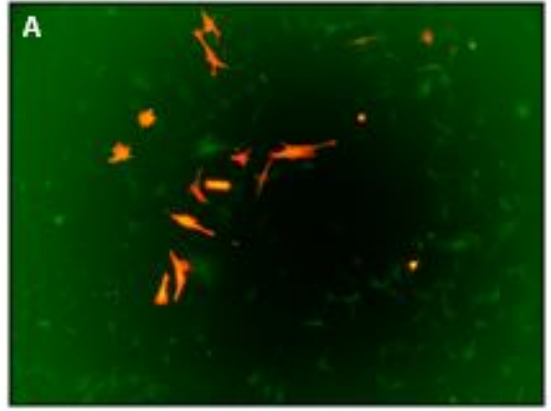

C
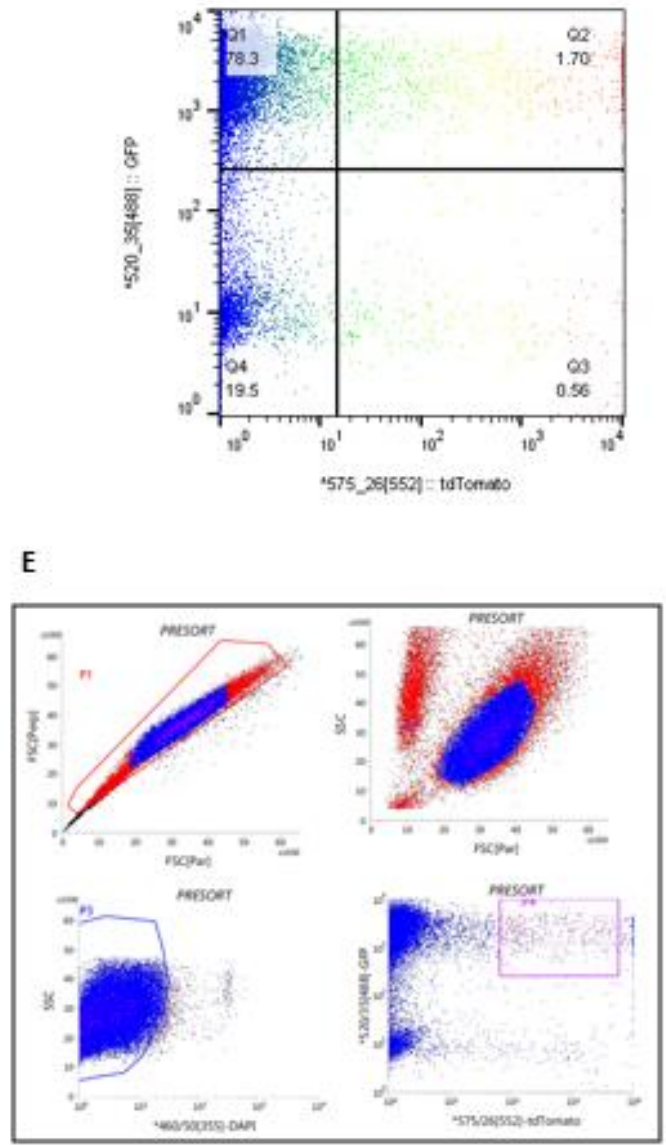

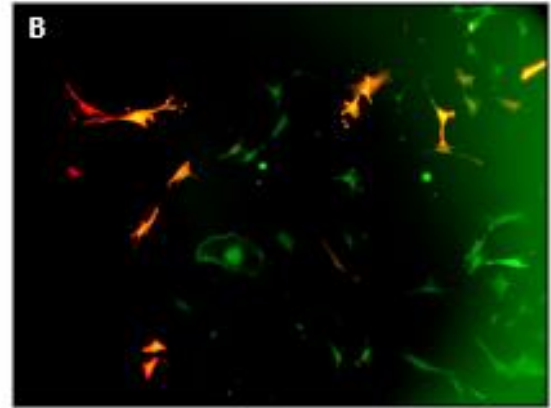

D

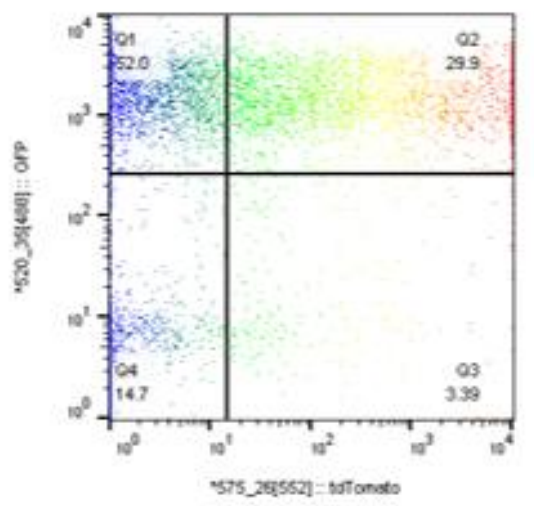

F

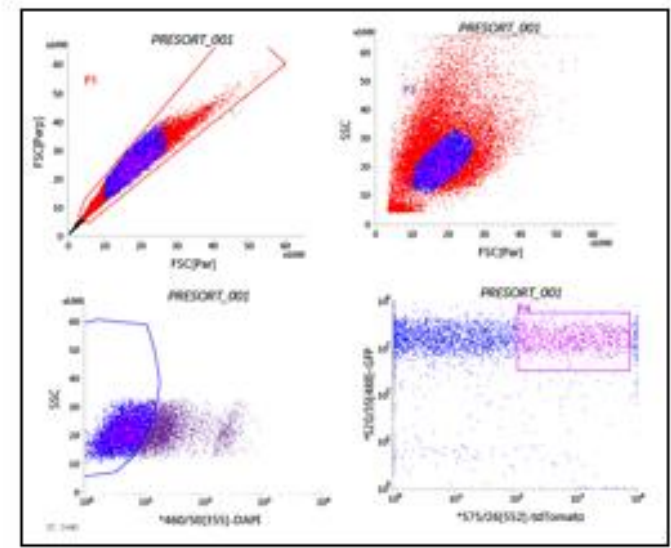

Figure 3.4: Co-transfection of pX330 plasmids and tdTomato. A \& B. Fluorescent microscope image of transfected astrocytes with FITC and TRITC filters overlaid (A. pX330 Pten and B. pX330 P53). C \& D. Flow cytometry results for GFP and tdTomato fluorescence. Quartiles show percentages of total cells analysed (C. pX330 Pten and D. pX330 P53). E \& F. FACS plots with gating strategy for isolating knockout cells. Single, DAPI negative, EGFP-tdTomato double-positive cells were sorted, indicated by the pink square (E. pX330 Pten and F. pX330 P53). 


\subsubsection{Culturing the bulk population of transfected cells}

In order to decrease the stress these cells experienced due to the transfection and sorting process, these bulk sorted cells were cultured in a single well of a 6 -well plate for two days. After two days of incubation, these cells were lifted into a new flask and allowed to grow to confluence. After approximately $4 \times 10^{6}$ cells had proliferated, the cells were harvested for DNA extraction.

\subsubsection{Culturing the clonal population of transfected cells}

After the single cells were sorted, they were incubated for two weeks, in order to give sufficient time for the cells to grow into a colony. From this point, a plan was developed through which colonies were strategically chosen to screen further. The two criteria for this decision were a) the colony size and b) the proportion of senescent cells which were in the colony. A trend was seen which split the colonies into three groups: group 1 had limited growth seen with high levels of senescence; group 2 had a moderate degree of growth but had visible levels of senescent cells; and 3 had the highest amount of proliferation and little to no signs of senescence (Fig 3.5A).

Group three of the colonies were lifted and passaged into a well of a 24 -well plate, for each mutation. The Pten 96-well plate contained a higher proportion of group 3 cells $(32.3 \%)$ than the P53 plate (22.9\%). However, the number of wells with no growth, indicating no survival from the sorting process, were also higher in the Pten population (55.2\%) compared with those in the P53 plate (47.9\%) (Fig 3.5B). Following the move to a 24-well plate, the clonal populations were assessed for growth characteristics, in particular speed of growth and any abnormal growth patterns. An example of abnormal growth was cells which adhered on top of neighbouring cells rather than on the flask itself. Interestingly, in well C8 of the cells transfected with pX330 Pten, cells showed visibly increased proliferation and abnormal growth, however, these cells had lost the expression of the EGFP transgene. Because of this, these cells were not investigated further as the EGFP expression is a crucial factor of the GEM-CLeMs. 
Additionally, six Pten group 2 colonies were also passaged. This was to assess the effect that the higher proportion of senescent cells would have on the colonies' ability to be subcultured. These six populations failed to proliferate in the new plate; from this result, as the P53 experiment occurred later, only wells of rank 3 were investigated. 
A

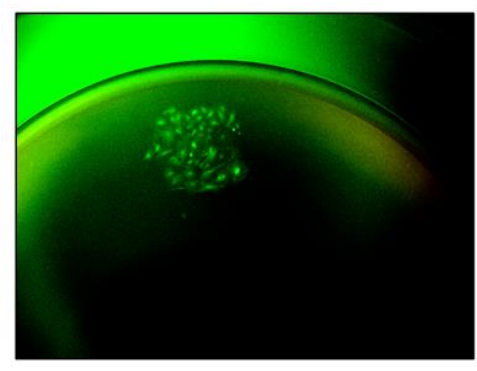

Group 1 - Minimal growth and high senescence

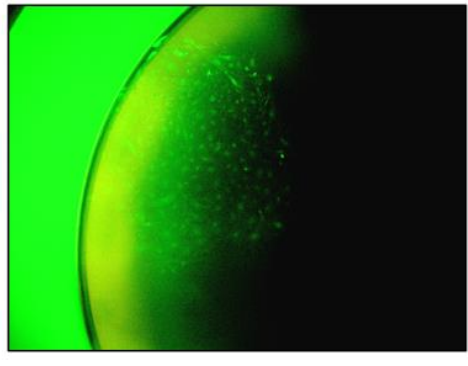

Group 2 - Moderate growth, mix of senescent and dividing cells

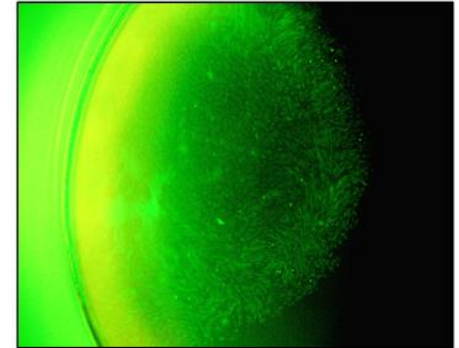

Group 3 - High levels of cell growth, minimal visible senescent

B

\begin{tabular}{|c|c|c|c|c|c|c|c|c|c|c|c|c|c|}
\hline & & 1 & 2 & 3 & 4 & 5 & 6 & 7 & 8 & 9 & 10 & 11 & 12 \\
\hline & $A$ & $\mathrm{x}$ & 3 & $\mathrm{x}$ & 3 & $\mathrm{x}$ & 3 & $\mathrm{x}$ & $\mathrm{x}$ & $\mathrm{x}$ & 3 & 2 & $\mathrm{x}$ \\
\hline & $B$ & $\mathrm{x}$ & $x$ & $x$ & 3 & $\mathrm{x}$ & 3 & 2 & $\mathrm{x}$ & 3 & $\mathrm{x}$ & 3 & 1 \\
\hline & C & $x$ & $x$ & 3 & $\mathrm{x}$ & 1 & $\mathrm{x}$ & 1 & 3 & 3 & $\mathrm{x}$ & $\mathrm{x}$ & 3 \\
\hline \multirow[t]{5}{*}{ pX330 Pten } & $D$ & $\mathrm{x}$ & 3 & $\mathrm{x}$ & $\mathrm{x}$ & $\mathrm{x}$ & 3 & $\mathrm{x}$ & $\mathrm{x}$ & $\mathrm{x}$ & 3 & $\mathrm{x}$ & 3 \\
\hline & $E$ & $\mathrm{x}$ & 3 & $\mathrm{x}$ & $\mathrm{x}$ & $\mathrm{x}$ & $\mathrm{x}$ & $\mathrm{x}$ & 3 & 1 & $\mathrm{x}$ & $\mathrm{x}$ & 2 \\
\hline & $F$ & 2 & 1 & 3 & 1 & $\mathrm{x}$ & $\mathrm{x}$ & 3 & $\mathrm{x}$ & 3 & $\mathrm{x}$ & $\mathrm{x}$ & $\mathrm{x}$ \\
\hline & $G$ & $\mathrm{x}$ & 3 & $\mathrm{x}$ & 3 & 3 & $\mathrm{x}$ & 3 & $x$ & 3 & $x$ & 3 & $\mathrm{x}$ \\
\hline & $H$ & 2 & 3 & $\mathrm{x}$ & 3 & $\mathrm{x}$ & $\mathrm{x}$ & 2 & $\mathrm{x}$ & $\mathrm{x}$ & 3 & 3 & $\mathrm{x}$ \\
\hline
\end{tabular}

\begin{tabular}{cc|cccccccccccc} 
& & 1 & 2 & 3 & 4 & 5 & 6 & 7 & 8 & 9 & 10 & 11 & 12 \\
\cline { 2 - 11 } pX330 P53 & 1 & 3 & 3 & 3 & $\mathrm{x}$ & 3 & $\mathrm{x}$ & $\mathrm{x}$ & 3 & 3 & 3 & $\mathrm{x}$ \\
$B$ & $\mathrm{x}$ & 2 & $\mathrm{x}$ & $\mathrm{x}$ & 3 & $\mathrm{x}$ & 3 & $\mathrm{x}$ & 2 & 3 & $\mathrm{x}$ & $\mathrm{x}$ \\
& $C$ & $\mathrm{x}$ & $\mathrm{x}$ & $\mathrm{x}$ & 2 & $\mathrm{x}$ & $\mathrm{x}$ & 3 & 3 & $\mathrm{x}$ & $\mathrm{x}$ & $\mathrm{x}$ & 2 \\
& $D$ & $\mathrm{x}$ & 3 & 3 & 2 & $\mathrm{x}$ & 3 & 1 & $\mathrm{x}$ & $\mathrm{x}$ & $\mathrm{x}$ & $\mathrm{x}$ & 2 \\
& $E$ & 3 & $\mathrm{x}$ & $\mathrm{x}$ & $\mathrm{x}$ & $\mathrm{x}$ & $\mathrm{x}$ & 3 & $\mathrm{x}$ & 2 & 1 & $\mathrm{x}$ & 2 \\
& $F$ & 3 & 3 & 3 & $\mathrm{x}$ & 2 & 3 & 3 & 3 & 1 & 2 & $\mathrm{x}$ & 1 \\
$G$ & $\mathrm{x}$ & $\mathrm{x}$ & $\mathrm{x}$ & 3 & $\mathrm{x}$ & $\mathrm{x}$ & 1 & 2 & $\mathrm{x}$ & $\mathrm{x}$ & 3 & 2 \\
& 1 & $\mathrm{x}$ & $\mathrm{x}$ & $\mathrm{x}$ & 2 & 2 & 1 & 3 & $\mathrm{x}$ & $\mathrm{x}$ & $\mathrm{x}$ & $\mathrm{x}$
\end{tabular}

Figure 3.5: Culturing and assessing clonal populations. A. Clonal colonies were seen in three groups determined by presumed levels of senescence and cell growth. $\mathbf{B}$. 96-well plates screened for growth groupings (1-3) and no growth (x) for pX330 Pten and P53 sorted cells. 


\subsection{Confirmation of CRISPR induced mutations}

\subsubsection{Heteroduplex assay}

The first analysis conducted was to investigate whether any indel mutations could be identified from the bulk populations. The Surveyor ${ }^{\circledR}$ heteroduplex assay was used to test this. Surveyor ${ }^{\circledR}$ is an endonuclease which is designed to make double strand cuts when a mismatch-loop is present (Fig 3.6A). This is useful when detecting mutations caused by CRISPR/Cas9, which introduces either an addition of one to three base pairs, or the deletion of up to 20. Importantly, this mutation occurs independently on each chromosome. As a result, while the heteroduplex assay is unable to distinguish between a heterozygous or a homozygous mutation, if an indel occurs on both chromosomes independently, a mismatch-loop will still be detected.

As a proof of concept experiment, a PCR product with a known mismatch loop was created across a known polymorphism in the mitochondrial tRNA-arginine gene. This gene contains a polyadenine $(\operatorname{Poly}(\mathrm{A}))$ sequence which varies between strains of mice and murine cell lines. The PCR product from the astrocytes contained 8A while the amplicon from the murine cell line, C1498, contained 11A. When denatured and rehybridised together, the PCR products with the variants contain a three bp mismatch loop, leading to fragmentation when digested with Surveyor ${ }^{\circledR}$ (Fig 3.6B). This showed that the heteroduplex assay could successfully identify indels. 

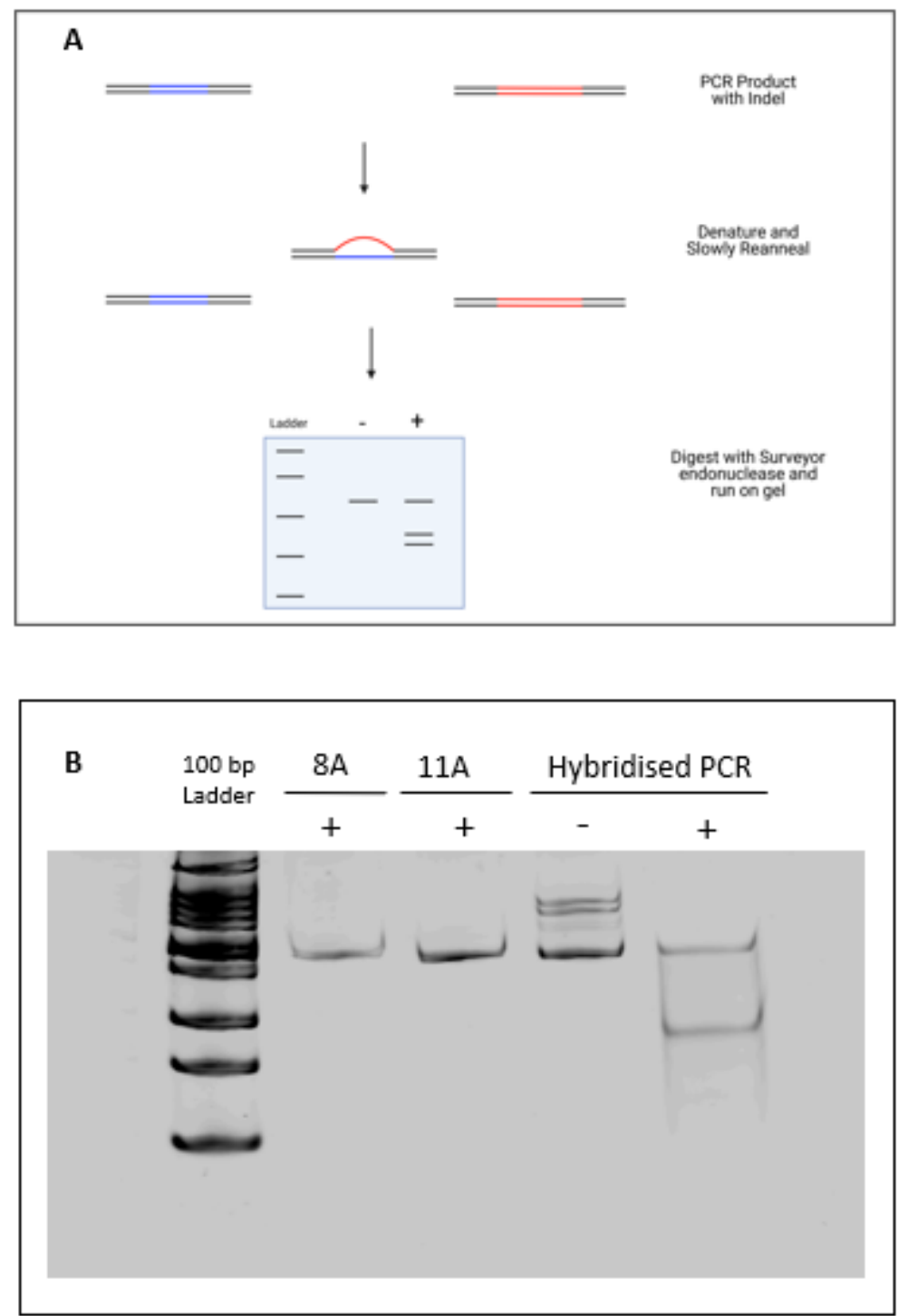

Figure 3.6: Methodology for the heteroduplex assay. Surveyor digested products $(+)$ and nondigested product (-). A. Schematic for mismatch loop formation when an indel is present. B. Proof of concept heteroduplex assay. Homoduplexes with central 8A and 11A sequences, and hybridised PCR products with mismatch loops. 


\subsubsection{Heteroduplex assay for the Pten locus}

The primers designed to target the Pten sgRNA binding sequence surround the loci asymmetrically. The forward primer is $64 \mathrm{bp}$ upstream of the theoretical cutting site while the reverse is $125 \mathrm{bp}$ downstream. Therefore, if indels were present in the bulk population, after the digestion by Surveyor ${ }^{\circledR}$ three bands would be seen: one at 189 bp representing the PCR product which correctly reannealed; and two fragmented bands representing the 125 and $64 \mathrm{bp}$ ends on either side of the sgRNA binding site. When the Pten loci was digested by Surveyor ${ }^{\circledR}$, the bulk population contained two faint fragments while the wildtype astrocytes did not (Fig 3.7A). This indicated that there were cells in the bulk population which contained an indel mutation inside the Pten gene, which was not present in the wildtype astrocytes.

To assess the Pten clonal colonies, three lines were initially selected as a pilot screen. Each line was named after the well from which they were sorted - C57B1, C57B3 and C57C3. These colonies were selected as they showed the fastest rate of growth compared with the other colonies. From these three colonies, only the C57C3 cells appeared to show signs of an indel mutation in the locus (Fig 3.7B). This result was confirmed by repeating the heteroduplex assay with a fresh DNA sample from C57C3 (Fig 3.6C). The cell line was renamed C57C3 Pten. 

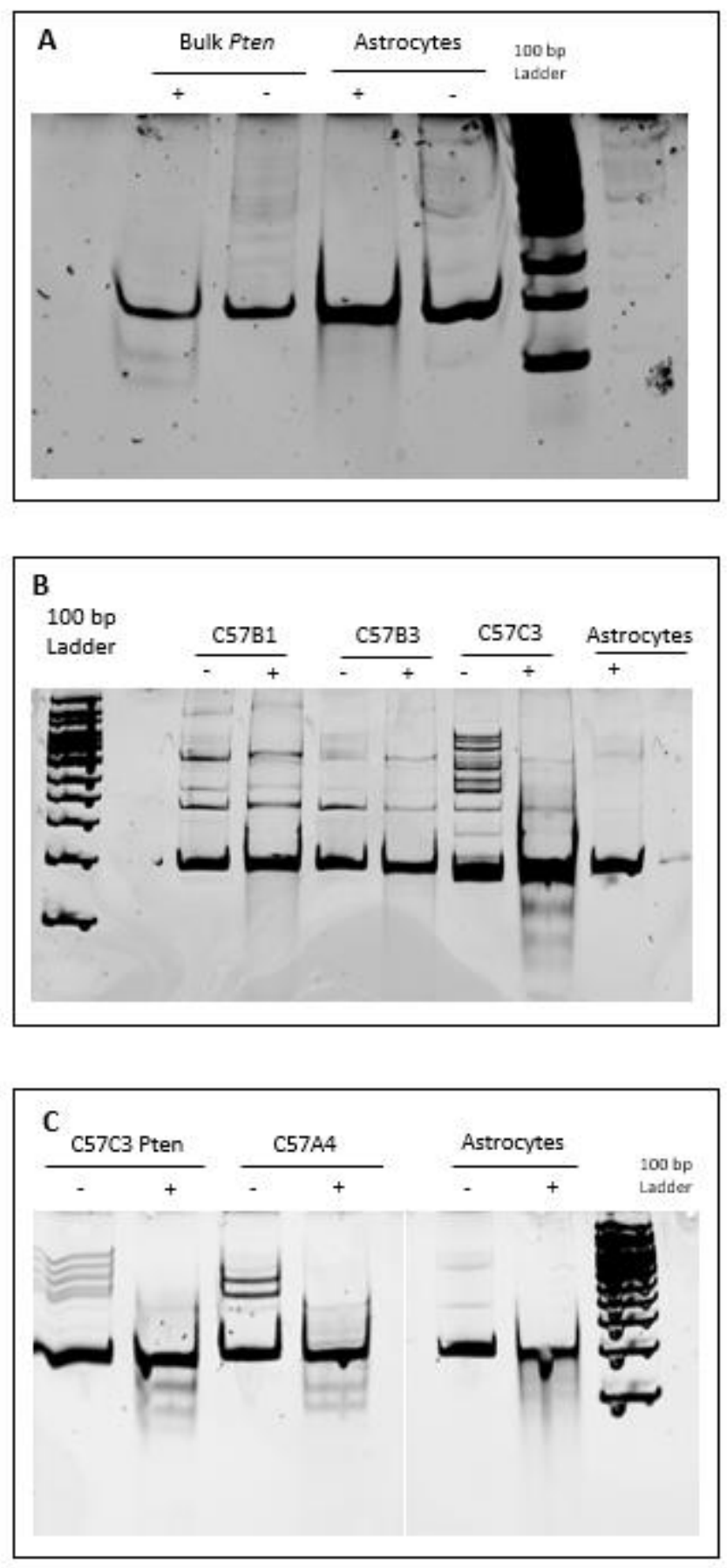

Figure 3.7: Heteroduplex screening for Pten indel mutations. Heteroduplex assays, Surveyor ${ }^{\circledR}$ digested $(+)$ and non-digested $(-)$, with astrocytes DNA as a negative control. A. Pten Bulk sorted heteroduplex assay. B. Initial screen for Pten sorted colonies C57B1, C57B3 and C57C3. C. Heteroduplex assay for C57C3 Pten line with C57A4 DNA as a positive control 


\subsubsection{Heteroduplex assay for the P53 locus}

As with the Pten loci, the primers surrounding the P53 sgRNA binding site were designed asymmetrically, resulting in a predicted fragmentation of the PCR product at 288 bp, and two fragmented bands at $135 \mathrm{bp}$ and $153 \mathrm{bp}$ respectively. After the Surveyor ${ }^{\circledR}$ digestion of the P53 population, with DNA from C57C3 Pten used as a positive control for digestion and astrocytes DNA as a negative control, the predicted fragmentation was observed (Fig 3.8A). Comparing the intensity of the fragments with the correctly annealed bands indicated that there were approximately equal proportions of each. This suggested that compared with screening for Pten indels, a greater percentage of the P53 clonal cell lines would contain a mutation in the P53 locus.

Five P53 colonies were selected for the heteroduplex assay - C57B4, C57E7, C57F3, C57G4 and C57H8. C57F3 was the only colony which showed signs of an indel mutation. This was confirmed by repeating the heteroduplex assay using new DNA from the $\mathrm{C} 57 \mathrm{~F} 3$ line, and the C57C3 Pten line was used as a positive control (Fig 3.8B). This confirmed that C57F3 possessed an indel mutation in the P53 sgRNA locus. The cell line was renamed C57F3 P53. 

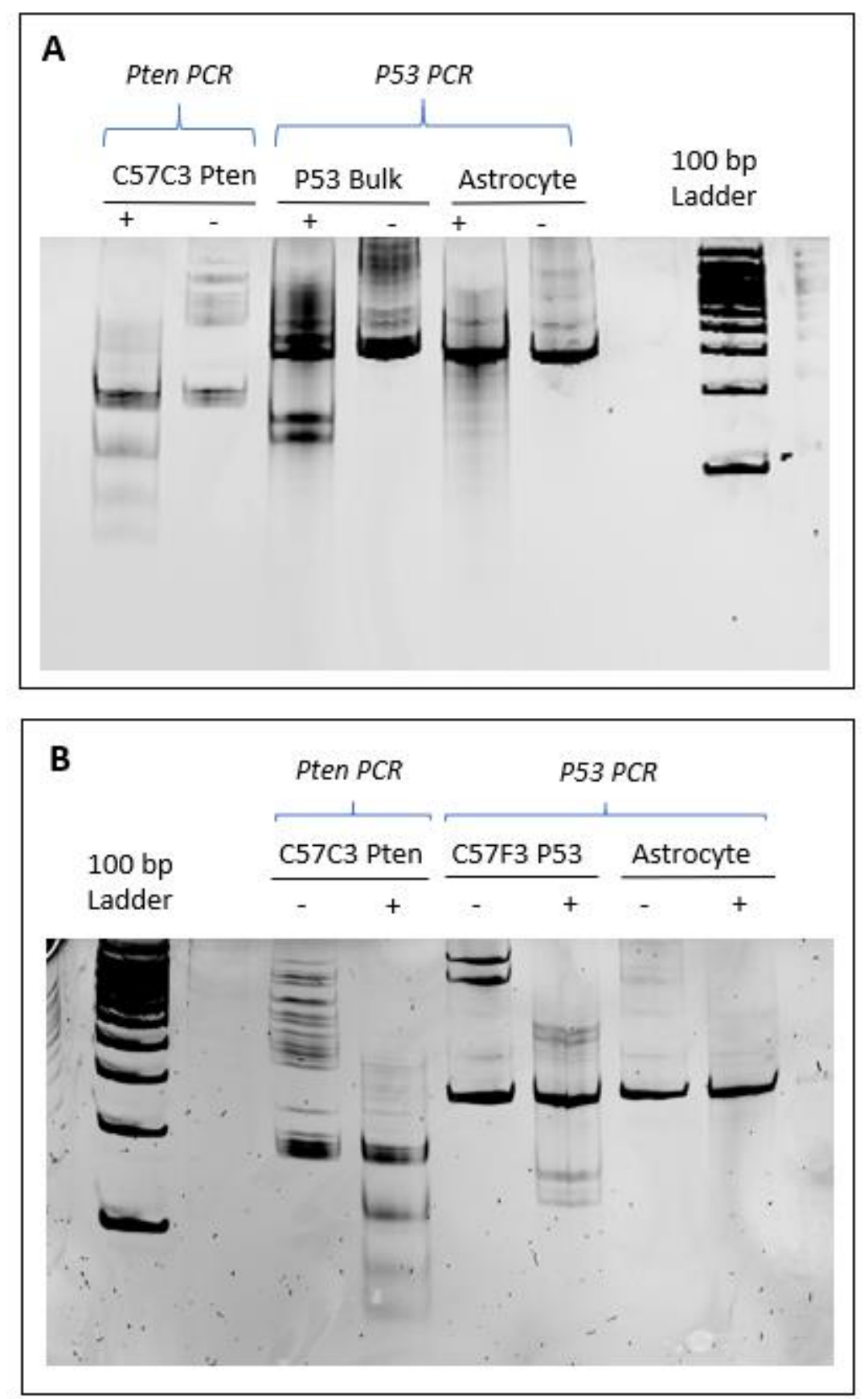

Figure 3.8: Heteroduplex screening for P53 indel mutations. Heteroduplex assays, Surveyor ${ }^{\circledR}$ digested $(+)$ and non-digested (-), with astrocytes DNA as a negative control and C57C3 Pten DNA as a positive control. A. Heteroduplex assay P53 bulk sorted. B. Heteroduplex assay to screen the C57F3 P53 line. 


\subsubsection{Oxford Nanopore sequencing}

To complement the confirmation of an indel in the sgRNA binding sites, sequencing data was generated in order to visually interpret what mutation had been introduced. The Sanger sequencing that confirmed the sgRNA binding sequence (3.1) required very little depth of coverage. However, it was not guaranteed that both alleles for these tumour suppressors had been cut by the Cas9 enzyme. Since the NHEJ repair mechanism occurs independently for each chromosome ${ }^{87}$, a sequencing technique with high coverage was required to separate the PCR products from each allele. Therefore, Oxford Nanopore MinION was used to sequence PCR amplicons of the potential indel mutations.

The Pten and P53 PCR products were barcoded, specific to each product, and pooled for the addition of MinION specific adaptor sequences. This allowed the two products to be sequenced simultaneously and then demultiplexed according to their barcodes. Once aligned to the mouse GRCm38/mm10 reference sequence, the presence of indels was assessed. The Pten amplicon showed an average depth of coverage of 9,000 reads. The sequencing results showed three separate indel in the alignment to the reference genome, one of $23 \mathrm{bp}$, one of $11 \mathrm{bp}$ and another of $4 \mathrm{bp}$ at the sgRNA binding site (Fig 3.9B). This is seen through a lack of coverage over this locus in the sashimi plot when mapped to the reference (Fig 3.9A). This confirmed that there was an indel in one of the chromosomes for the Pten gene, as the remaining amplicons were full length. Comparatively, there was a cleaner consensus for the indel in the P53 gene, which had an average depth of coverage of 16,500 reads. A 15 bp gap was seen at the Lys ${ }^{15}$ position in approximately half the amplicons (Fig 3.9C); this is predicted to result in a 5 amino acid deletion, in frame with the codons (Fig 3.9D). 
A

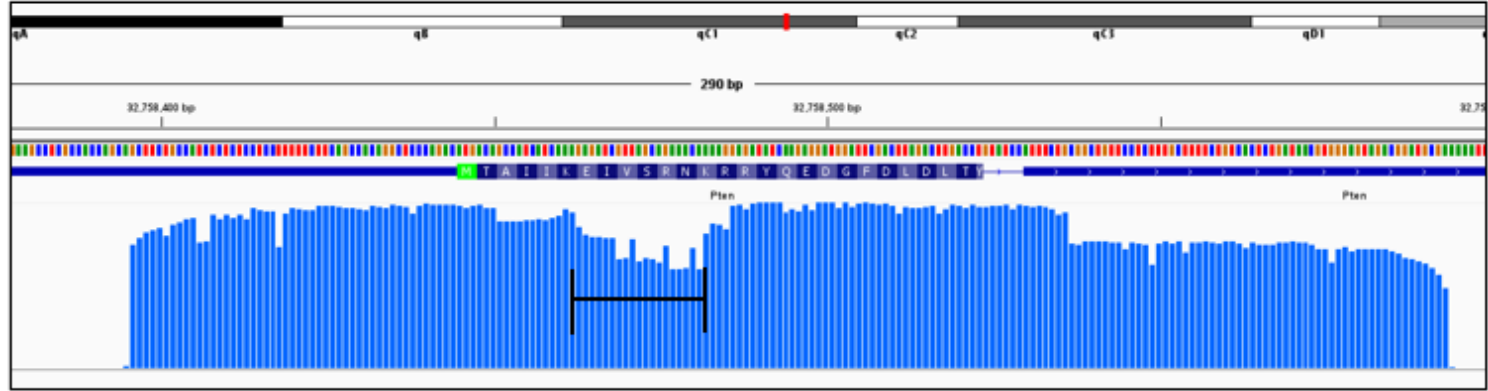

Original Pten MTAIIKEIVSRNKRRYQEDGFDLDLTY

23 bp Indel Pten MTAIIK___ EYSRGWIRLRLD

11 bp Indel Pten MTAIIKEI__SKGDIKRMDS*

4 bp Indel Pten MTAIIKEIVS_RKGDIKRMDST*

C

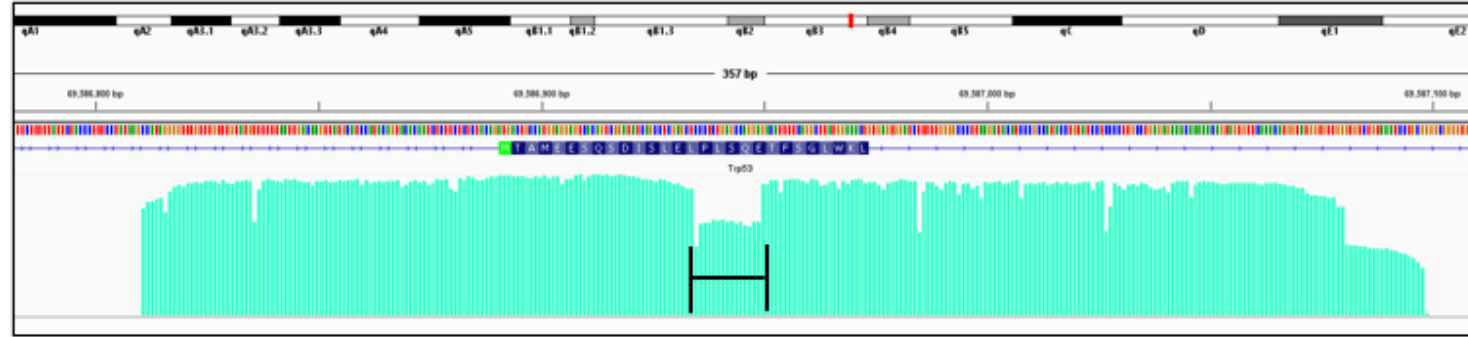

D

Original P53 MTAMEESQSDISLELPLSQETFSGLWKL

15 bp Indel P53 MTAMEESQSDISLE___ LTFSGLWKL

Figure 3.9: Oxford Nanopore MinION sequence confirmation of indels. Gap (_) and stop codon $\left(^{*}\right)$. A. Sashimi plots for sequence consensus of C57C3 Pten PCR against the GRCm38/mm10 mouse reference sequence. Brackets indicate the indel location. B. Predicted Pten protein coding sequence for C57C3 Pten. C. Sashimi plots for sequence consensus of C57F3 P53 PCR against the GRCm38/mm10 mouse reference sequence. Brackets indicate the indel location. D. Predicted P53 protein coding sequence for C57F3 P53. 


\subsubsection{Confirmation of Pten loss by western blot}

Western blot analysis was used to detect whether the indel in the C57C3 Pten line resulted in a corresponding lack of expression of the Pten protein. The lysate from the astrocytes were compared with that of C57C3 Pten. A significant decrease in Pten protein was observed when compared with the astrocyte's lysate (Fig 3.10A). This result confirmed that the indel in the Pten gene resulted in a change in abundance of Pten protein. This change, however, does not appear to be a full knockout of the protein, as there is still a visible band seen on the western blot. This result was consistent with the sequencing data, as an indel was predicted to affect protein expression.

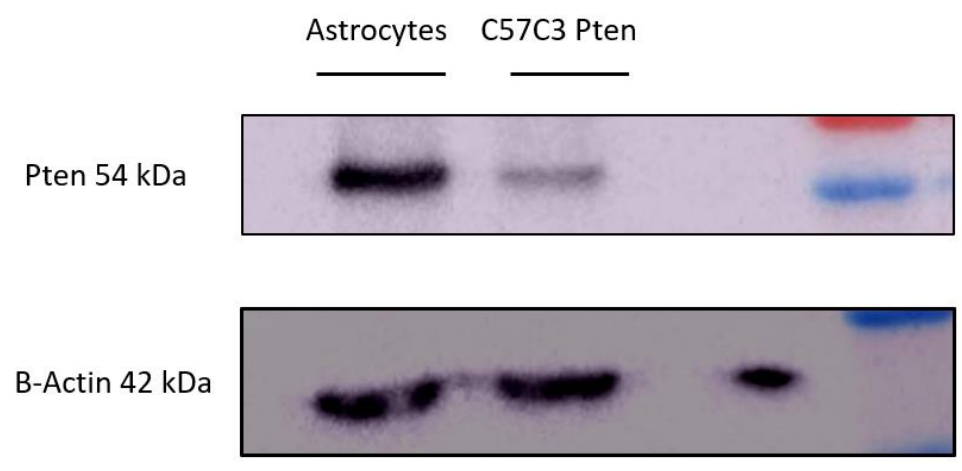

Figure 3.10: Western blot for Pten protein abundance. Western blot for Pten using lysates from astrocytes and C57C3 Pten cells with $\beta$-actin as a loading control

\subsubsection{Confirmation of P53 loss by western blot}

Once it was established that the C57F3 P53 cell line had an indel in the sgRNA locus, a western blot for P53 was conducted. P53 levels are strictly regulated inside the cell, with an approximate half-life of 20 minutes before ubiquitin-directed degradation. Additionally, the protein Mdm2 regulates P53 through physically binding to an allosteric regulatory loop, leading to inhibition of P53s DNA binding and transcriptional roles 106108. P53 becomes activated and increases in abundance through phosphorylation, which stabilises the protein; this response is activated through the DNA break repair pathway ${ }^{108}$. 
In order to assess the true abundance of protein via western blot, ionising radiation was given to the C57F3 P53 and astrocyte cell lines. As had previously been established in our lab, 2 Gy of ionising radiation was sufficient to stabilise P53.

The western blot showed the appropriate relative difference in P53 abundance between irradiated and non-irradiated cells, confirming appropriate regulation of P53 in the astrocytes. The blot also indicated that there had been a significant decrease in P53 protein expressed by the C57F3 P53 cell line compared with the astrocytes (Fig 7D).

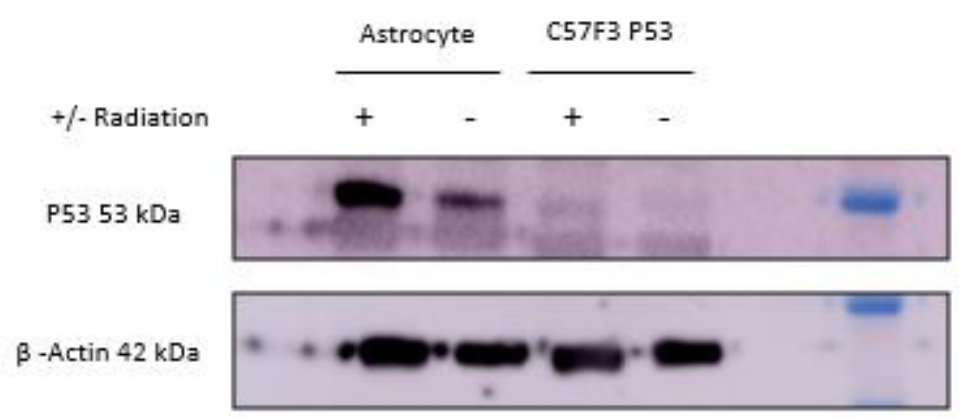

Figure 3.11: Western blot for P53 protein abundance. Western blot using astrocyte and C57F3 P53 protein lysate for P53 with $\beta$-actin as a loading control. Irradiated $(+)$ and nonirradiated (-) protein lysates were used.

\subsection{G418 Kill Curve Assay}

In order for oncogenic mutations to be introduced to these cell lines a protocol for stable transfections was required. This process involved transfecting a plasmid which contained a gentamicin sulfate (G418) resistance cassette. Once transfected, a concentration of G418 would be given to the cells, those which have taken up the plasmid would express the plasmid and survive, and those which did not would die. However, in order to ascertain what concentration of G418 the astrocytes were naturally resistant to, a kill curve assay was developed. 
Using a range of concentrations from $0 \mu \mathrm{g} / \mathrm{ml}$ to $630 \mu \mathrm{g} / \mathrm{mL}$, the astrocytes were exposed to the drug over five days. After this, the cells were harvested and resuspended in $100 \mu \mathrm{L}$ of FACS buffer, to ensure the only variation in cell concentration was due to the number of live cells in response to the drug. Flow cytometry of these cells showed that the number of cells still expressing EGFP declined rapidly from $0 \mu \mathrm{g} / \mathrm{mL}$ to $360 \mu \mathrm{g} / \mathrm{mL}$, then the gradient began to plateau (Fig 3.8). From this data, the concentration which was chosen for future stable transfections was $500 \mu \mathrm{g} / \mathrm{mL}$. This was because from $450 \mu \mathrm{g} / \mathrm{mL}$ there are virtually no surviving cells. The slight move to $500 \mu \mathrm{g} / \mathrm{mL}$ allows for increased confidence that surviving cells had successfully been transfected.

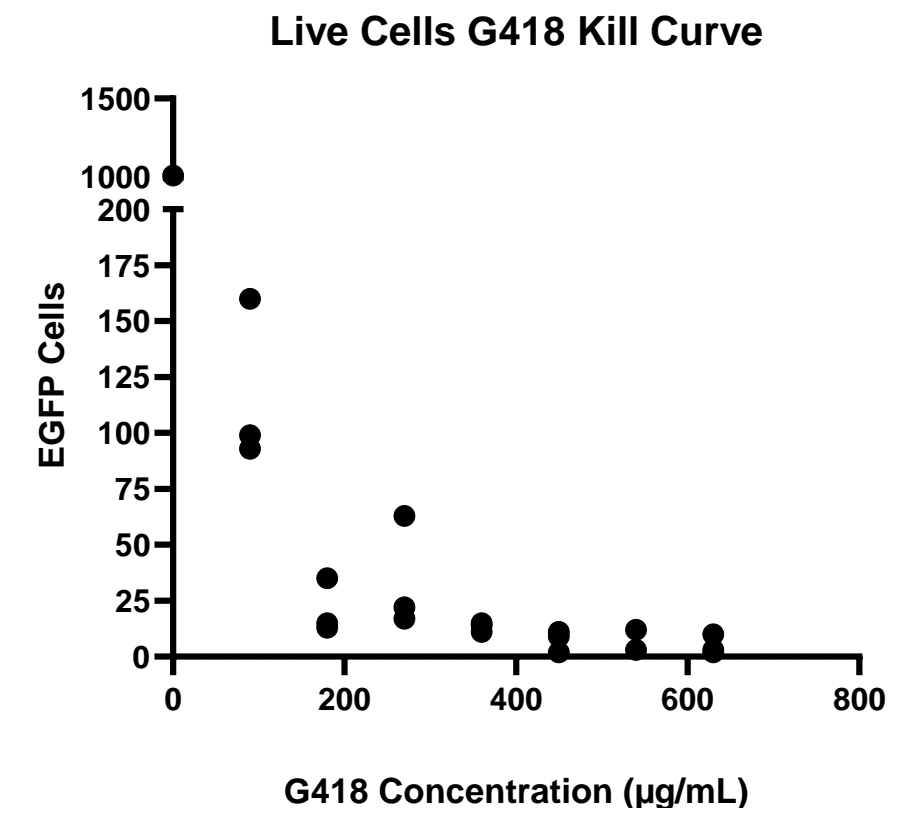

Figure 3.12: G418 drug kill curve. Kill curve for the number of cells expressing EGFP at a range of G418 concentrations. Data was collected with technical triplicates. 


\subsection{Discussion}

This chapter outlined the development of two cell lines, a Pten knockout EGFP astrocyte and a P53 knockout EGFP astrocyte. The plasmids used had been previously shown to generate the knockout mutations in mice liver cells ${ }^{101}$. The methods used for transfection were based upon those from this paper, such as co-transfection of tdTomato alongside the pX330 plasmids, and the Surveyor ${ }^{\mathbb{B}} /$ heteroduplex assay to confirm the presence of an indel mutation. The results correlate strongly with the literature available surrounding the generation of indels using simple dsDNA breaks 90,93,109,110. The initial study which utilised these plasmids did not look in depth into the indels produced, but instead used immunohistochemistry as the major experiment ${ }^{101}$. Because of this, I developed the MinION sequencing and western blot investigations to gain further information on the clonal cell lines.

Additionally, as Xue et al. (2014) used these plasmids in vivo, the culturing of clonal colonies was a protocol I developed. Through only screening group 3 colonies for heteroduplex formation, some selection bias may have been introduced into the process. Two factors influenced this decision; the effect the tumour suppressor knockouts would have on the cells, and the proportion of senescent cells present. Pten directly inhibits a cell's proliferative and growth ability. Therefore, if the colonies were developed from a Pten mutant cell, it would therefore be anticipated that an immediate proliferation advantage would be seen when cultured. I observed that cells which grew to the size of group 3 had a visibly faster growth after culturing in the 96-well plate. As mentioned, group 2 cells were investigated further, yet continued growth was not achieved. This was largely due to the high proportion of senescent cells, which led to difficulties in the colonies growing to confluence. As the optimisation for this protocol was conducted while developing the Pten line, this assumption of greater growth correlating with a likelihood of a successful mutation was again made while developing the P53 mutations. However, P53 does not influence mitotic pathways as heavily as Pten. Because of this, a selection bias may have been present; if this protocol were to be replicated in the future, a more in-depth investigation into the mutational status of group 2 colonies could be conducted. Although, 
for this project, the protocol developed allowed for a more streamlined process which was successful in identifying successful CRISPR/Cas9 mutations.

Another potential technique is the creation of large deletions with two sgRNA cut sites and the removal of whole or multiple exons. These deletions result in a higher likelihood that the CRISPR/Cas9 mechanism would lead to either a loss of protein expression or a truncated protein which cannot function appropriately $93,111,112$. This technique may have led to an increased or complete protein loss in this project. The western blot results showed that while there was a significant decrease in abundance of proteins, there was still expression. Single Cas9 cuts have the potential to introduce very small indels. If the mutation introduced leads to a silent or a synonymous amino acid replacement, there may be limited effect on the protein produced. Larger scale CRISPR/Cas9 mutations through designing a second sgRNA for the tumour suppressors could be a potential future avenue to explore ${ }^{88}$.

The potential for CRISPR/Cas to induce off-target mutations has been well documented ${ }^{113}$. While the necessity of a PAM site immediately adjacent to the sgRNA binding sequence decreases the potential for the sgRNA/Cas9 complex to bind elsewhere in the genome, it is not impossible. Xue et al. (2014) published four primer sets for the predicted off-target sites, which was calculated by their similarity to the target region ${ }^{101}$. It was not within the scope of this project to analyse these sites. However, future investigations into these cell lines should assess whether off-target mutations have been made in the C57C3 Pten and C57F3 P53 cells. This would be achieved through the same protocols I have optimised in this chapter, and using the primers designed by Xue et al.

Through the combination of MinION sequencing and western blot, I was able to determine how the mutations affected the genes of interest. In the C57F3 P53 line, the 15 bp deletion meant that a frameshift mutation was not introduced to exon one. This five amino acid deletion was not guaranteed to result in a structural or a functional change to the P53 protein. However, through the decrease in abundance at baseline levels and after DNA damage, we can see that there has been a functional change with regards to either the transcription of the P53 mRNA or the translation of the protein. There are also two 
additional factors that can be considered in assessing the outcome of the P53 indel: the location of the mutation; and the requirement for P53 to act as a tetramer.

Exon one of P53 codes for the transactivation domain (TAD1), which is known to be essential for DNA damage response pathways. This domain functions by recruiting general transcription factors to aid P53 in the transcription of proteins which will either repair the damage or arrest the cell cycle ${ }^{114}$. Even without a frameshift, a five base pair deletion to this locus has the potential to hinder this function of the protein. Additionally, because P53 acts as a tetramer, this mutation has the potential to induce a dominant negative effect when paired with the wildtype corresponding allele, where the protein produced by the mutant allele blocks the function of the protein as a whole. While it was not within the scope of this project to investigate the structural consequences these mutations have introduced, this is a possible experiment which could be explored.

The MinION data generated for the Pten indel did not provide much information on the consequence of the indel on the protein. Three different consensus sequences were generated, each resulting in a different outcome to the predicted translated protein. The largest indel was a $23 \mathrm{bp}$ deletion after the aspartic acid at position seven. This indel would result in a seven amino acid deletion and a frameshift downstream (Fig 3.9B). The 11 and four bp deletion, while smaller, show potentially a much greater functional change to the Pten protein. These indels result in a three and two amino acid deletion, respectively, and a frameshift downstream. However, the most important functional consequence of this mutation is the introduction of a termination codon $12 \mathrm{bp}$ after the deletion (Fig 3.9B). This result is important because it introduces two possible outcomes which could explain the result seen in the Pten western blot.

The first potential result is that the indel may have led to nonsense mediated decay (NMD). This is a mechanism cells possess which results in the degradation of a protein as it is being translated, if a premature stop codon is processed ${ }^{115}$. A proposed method of NMD is through the ribosomal sensing of exon-junction complexes (EJCs). When a premature stop codon is sensed by the ribosomes while downstream an EJC remains in contact with the transcript, NMD is believed to be activated. This is because if two exons are spliced together after the stop codon, it is unlikely that the stop codon is truly signalling 
the end of the protein ${ }^{115-118}$. NMD is important as it decreases the possibility that a truncated form of the protein could be expressed with an altered mode of action. If this were the case, the transcripts from the mutated Pten allele would not result in any protein expression.

A second potential outcome of this mutation is that transcripts from the allele with the premature stop codon are translated. This would be predicted to result in a Pten protein which is made up of solely exon one would be produced. As a result, the antibody for Pten would not recognise the truncated protein, as the epitope the antibody recognises is at amino acid position 138.

Both theories would result in a western blot showing a decrease in Pten abundance. As exon one is not the functional phosphatase domain, it is unlikely that a truncated form of the protein would have a tumour suppressor role. Therefore, I am confident that the results of the screening process have isolated and characterised a cell line with a significantly decreased Pten abundance and activity.

\subsection{Conclusion}

Overall, two cell lines were generated through CRISPR induced mutations to exon one, C57C3 Pten and C57F3 P53. These cell lines showed a significant decrease in the abundance of protein produced, which was assessed through western blot. Future proteomic analysis could investigate the nature of the mutated proteins and whether the affected allele is expressed in these cell lines. The protocols I developed showed a consistent and reliable pipeline which can be used for producing future CRISPR induced mutations. Following this, these cell lines were used as a basis for the GBM models: the 'classical-like' and the 'secondary-like' GEM-CLeMs. 


\subsection{Development and Characterisation of the 'Classical-Like' GEM-CLEMs}

\subsection{Introduction}

The classical subtype of GBM is characterised by its extremely proliferative and invasive nature, largely due to the mutations and upregulation of drivers of the EGFR/MAPK pathway. This chapter describes the development of the C57C3 Pten line into a 'classical-like' GEM-CleM through the separate addition of constitutively active forms of MAPK drivers: EGFRVIII and RAS V12. Following this, the cell lines were compared with the astrocytes for cancer related changes in phenotypes, such as an altered proliferation, migration and metabolism.

\subsection{Addition of EGFRVIII and RAS V12 Oncogenes to C57C3 Pten}

\subsubsection{EGFRVIII and RAS V12 plasmids}

Two plasmids were obtained in order to introduce the EGFRVIII and RAS V12 oncogenes into the C57C3 Pten line. The PB-CMV-TO-EGFRvIII-IRES-nlsChe was a gift from Michael Elowitz ${ }^{102}$; this plasmid contained sequences for the human oncogene EGFRVIII, mCherry and EGFP fluorescent markers, and for G418 and ampicillin resistance cassettes (Fig 4.1A). The pcDNA3-H-Ras_V12 was a gift from Julian Downward 103; this plasmid contained sequences for the human oncogene RAS V12 and G418 and ampicillin resistance cassettes (Fig 4.1B). 

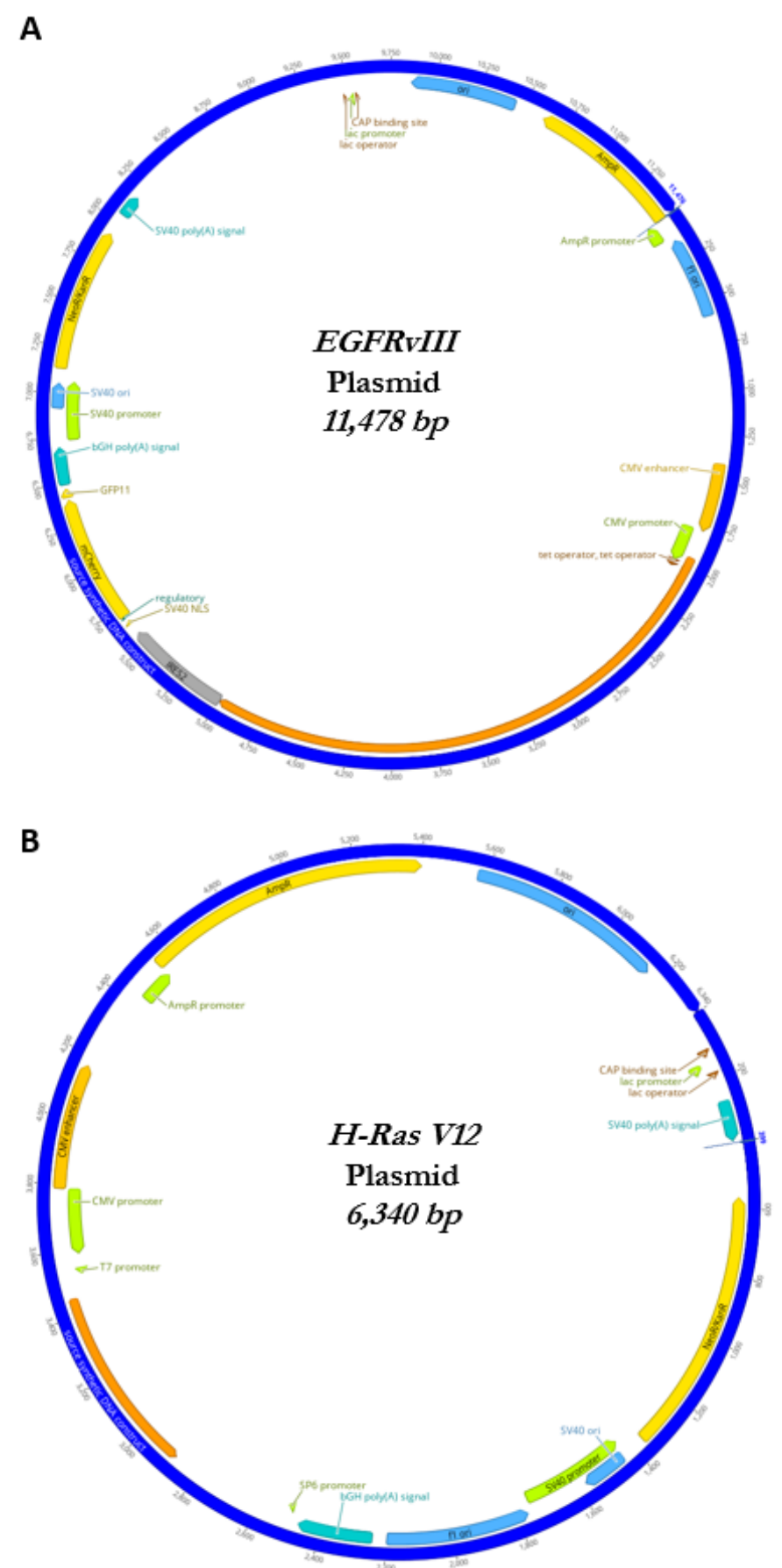

Figure 4.1: Oncogene plasmids transfected into the C57C3 Pten cell line. Plasmid maps for EGFRVIII and RAS V12 oncogenes. A. EGFRVIII plasmid; EGFRVIII (orange) expressed alongside mCherry and GFP gene with an IRES domain and a neomycin/kanamycin and ampicillin resistance cassettes (yellow). B. RAS V12 plasmid; RAS V12 gene (orange), neomycin/kanamycin and ampicillin resistance cassettes (yellow). 


\subsubsection{Stable transfection of EGFRVIII and RAS V12}

The C57C3 Pten cells were separately transfected with EGFRVIII and RAS V12. The EGFRVIII plasmid contained the sequence for mCherry (a red fluorescent marker) and EGFP. However, the mCherry signal was extremely weak and could not be seen visually using the fluorescent microscope. This lack of fluorescence was predicted to be due to the localisation of the markers to the nucleus, leading to a weak fluorescent emission compared with the cytoplasm localised EGFP transgene the astrocytes possess.

A separate transfection of tdTomato was used as a control for a successful transfection, because, unlike the oncogene plasmids, the fluorescence was visible within 24 hours, and a majority of cells were expressing the marker after two days. At this point G418 was delivered to the cells and incubated overnight. The tdTomato cells then acted as a negative control for $\mathrm{G} 418$ resistance, as without a resistance cassette, these cells would die with the $500 \mu \mathrm{g} / \mathrm{mL}$ concentration of G418. After two days in contact with G418, the remaining C57C3 Pten cells were lifted into a single well of a six-well plate and left to grow to confluence while fresh DMEM-G418 was supplied every two days. These cells were then lifted into a T75 and expanded, then cultured for two weeks before some were harvested for RNA extraction.

\subsubsection{Reverse transcription PCR to confirm oncogene expression}

In order to confirm whether the oncogenes were being expressed, reverse transcription (RT) PCR was conducted using cDNA produced from the RNA. Primers were designed for the beginning of the coding sequence for EGFRVIII and RAS V12. Each primer pair spanned approximately 200 bp and had no homology with the endogenous murine non-mutated version of the genes. Running the PCR products on a $1.5 \%$ agarose gel confirmed that the stable transfection of EGFRVIII and RAS V12 had been successful and the oncogenes were being expressed (Fig 4.2A \& B). The constitutively expressed gene, HPRT, was amplified as a positive control 

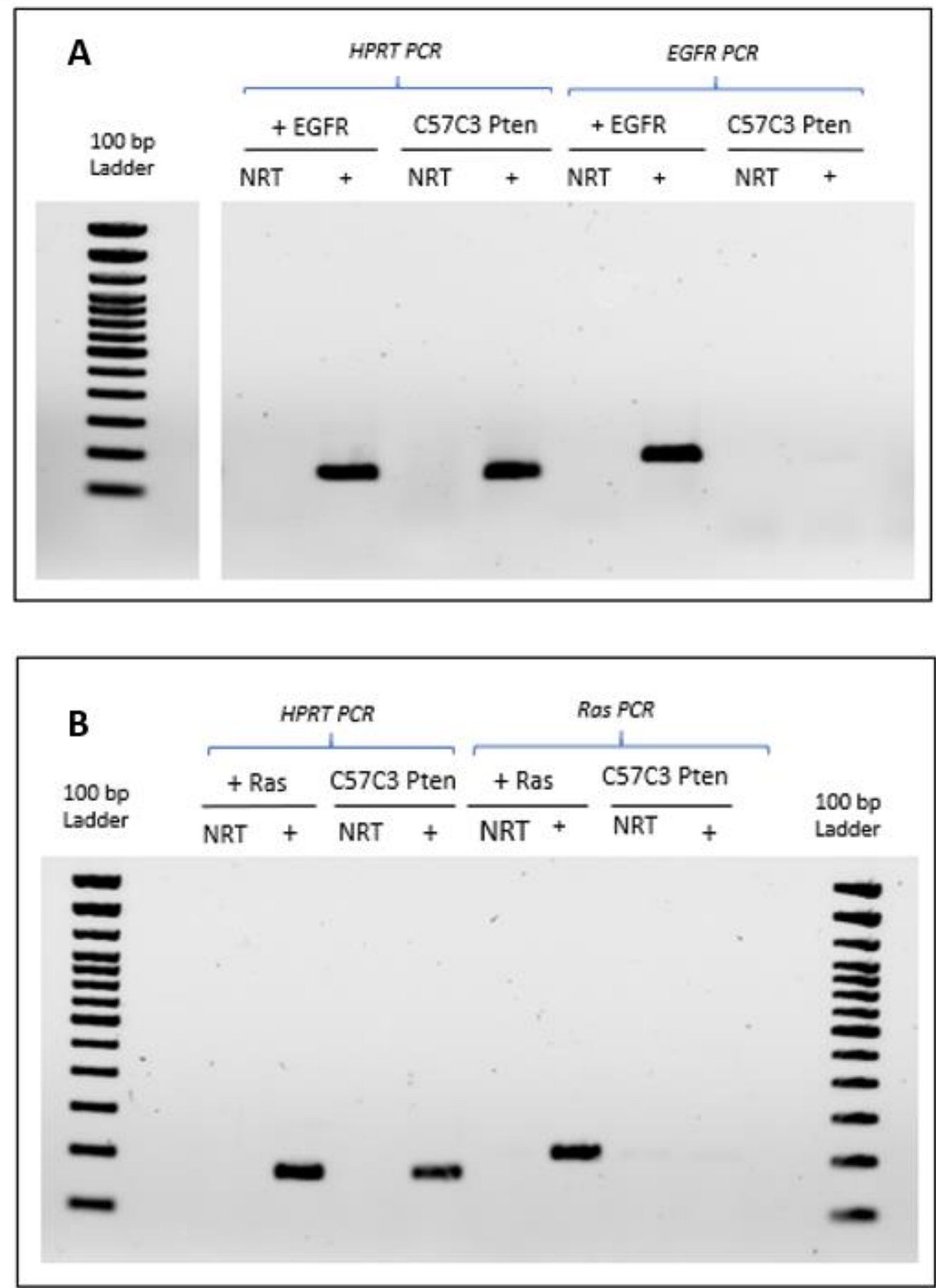

Figure 4.2: RT-PCR confirmation of oncogene stable transfections. 1.5\% TBEagarose gels with RT-PCR products for EGFRVIII and RAS V12 expression. HPRT was used as a positive control. A. EGFRVIII RT-PCR product from C57C3 Pten and Pten + EGFR RNA. B. RAS V12 RT-PCR product from C57C3 Pten and Pten + RAS RNA. 


\subsection{Functional Changes in the Akt/mTor Pathway}

\subsubsection{Phosphorylated Akt (pAkt)}

To complement the Pten western blot which confirmed the decrease in Pten expression (3.4.6), the functional consequences of this loss were assessed. Pten is an inhibitor of PI3K, which when active leads to the phosphorylation and activation of the effector protein, Akt. Akt has two regulatory phosphorylation sites: Serine 473 (Ser473) and Threonine 308 (Thr308). Mutations created using this guide sequence to knockout Pten have previously been shown to increase the abundance of Ser473 ${ }^{101}$. Because of this, the abundance of pAkt Ser473 was compared in the 'Classical-like' GEM-CLeMs.

Serum stimulation of the cell lines was used to observe the signalling pathway. Firstly, the cell lines were starved of serum to stop the phosphorylation of Akt and reduce background. This was followed by re-stimulation with serum to reinitiate the phosphorylation of Akt, then the abundance was measured after 15 minutes in contact with growth factors. The experimental design for this western blot included a non FBSstimulated control, to show the baseline levels of pAkt phosphorylation, and a PI3K inhibitor control (treated with BEZ235), to indicate the absence of pAkt phosphorylation.

\subsubsection{Western blot for pAkt}

A western blot comparing the protein lysate from C57C3 Pten and the astrocytes showed that the Pten knockout had a significant increase in pAkt Ser473 (Fig 4.3A). This confirmed that Pten had been knocked out of the C57C3 Pten cells. This was because an increased Akt phosphorylation implied that there was a loss of PI3K inhibition. After the addition of EGFR and RAS, this experiment was replicated. The results from this showed that both Pten + RAS and Pten + EGFR cell lines confer an additive effect; both lysates showed an increase in pAkt abundance, compared with the C57C3 Pten cells alone. Pten + EGFR showed a higher abundance of pAkt than Pten + RAS (Fig 4.3B). The protein 
loading across the gel was not even; this can be seen through the lower intensity seen in the loading control. However, the relative increase in pAkt was observed.

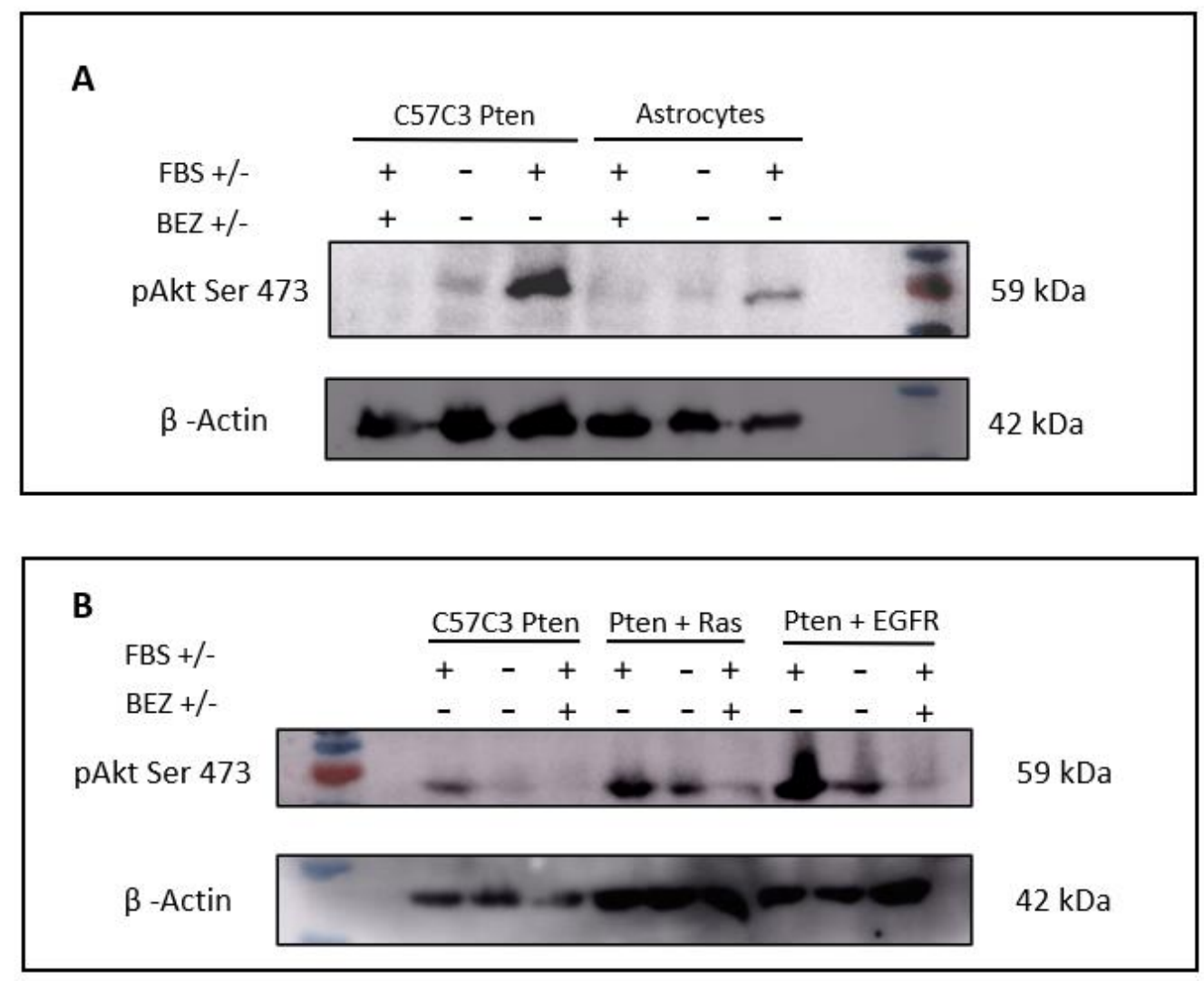

Figure 4.3: Western blot for pAkt in 'classical-like' cell lines. Western blot for pAkt Serine-473 with $\beta$-actin as a loading control. FBS stimulated (+) and non-stimulated (-), and BEZ 235 stimulated $(+)$ and non-stimulated $(-)$ controls. A. C57C3 Pten and astrocytes protein lysate. B. C57C3 Pten, Pten + RAS, and Pten + EGFR protein lysates. 


\subsection{Characterising Proliferation Rates}

\subsubsection{Incucyte microscopy}

Incucyte $^{\circledR}$ analysis was used to quantify how the cell lines grew over a time course. The Incucyte ${ }^{\circledR}$ is a device which consists of an incubator, set at $37^{\circ} \mathrm{C}$ with $5 \% \mathrm{CO}_{2}$, and a microscope camera. The Incucyte ${ }^{\circledR}$ allows for high quality images taken in a consistent position which leads to accuracy in assessing confluence and cell movement. Additionally, the Incucyte ${ }^{\circledR}$ has software which automates the process of calculating the data created through the experiments. This is done by training the software to identify the cell outline, so that confluence data can be gathered. In order to assess the proliferation rate of the cells, each line was plated at a low confluence (approximately 5\%) and images were taken every four hours over four days.

\subsubsection{Comparing C57C3 Pten and astrocyte proliferation rate}

The proliferation rate of the astrocytes was used as the baseline level of growth. When plated at 5\% confluence, it was seen that the astrocytes reached $80 \%$ confluence at 88 hours $(S E M=0.728, n=3)$. In comparison, the C57C3 Pten line reached the same level of confluence after 76 hours $(S E M=3.219, \mathrm{n}=3)$ (Fig 4.4A). The area-under-curve (AUC), as a measure of the rate of proliferation, was compared; the C57C3 Pten line showed statistically significantly higher average area, $t(6)=9.978, \mathrm{p}<0.0001 \quad(M=3562, S E M=90.24)$ compared with the astrocytes $(M=2909$, SEM=94.81) (Fig 4.4B). 
A

Growth Curve Comparison Astrocytes Vs C57C3 Pten

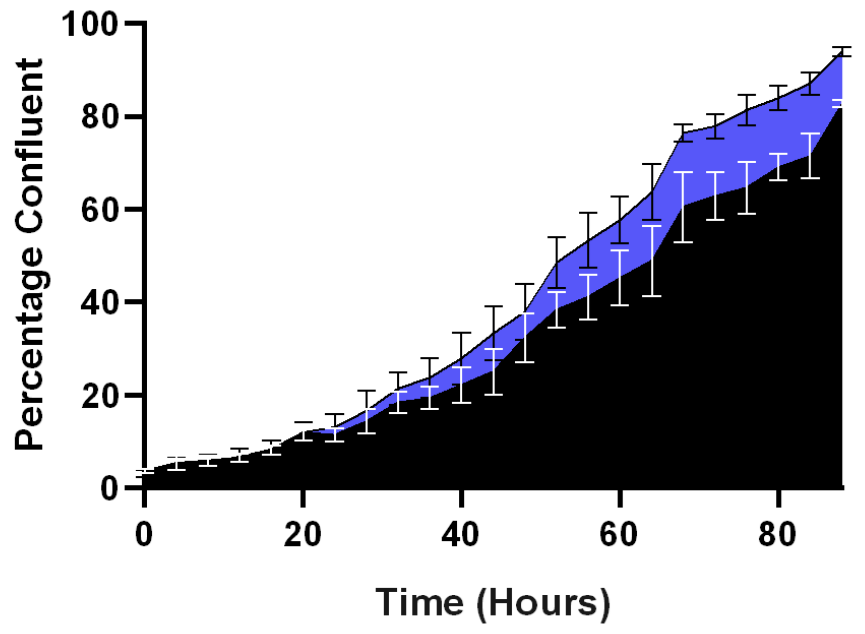

- Astrocytes

- C57C3 Pten

B

AUC of Growth Curve for Astrocytes Vs C57 C3 Pten

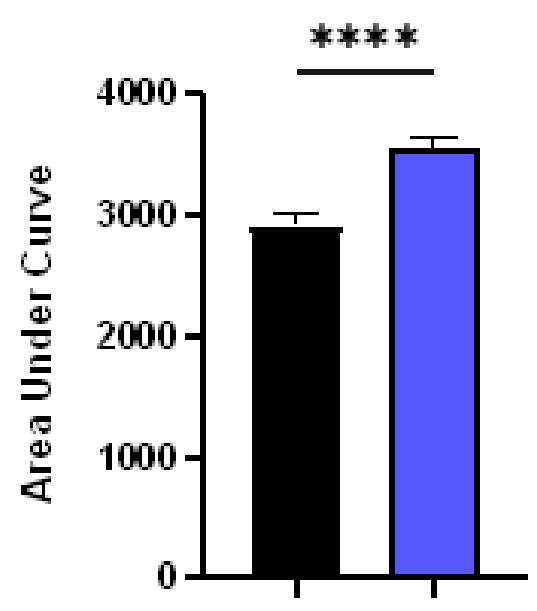

- Astrocytes

$\square$ C57C3 Pten

Cell Lines

Figure 4.4: Proliferation rate comparison for astrocytes and C57C3 Pten cells. Growth curve analysis for astrocytes (black) and C57C3 Pten cells (blue). Error bars represent SEM, n=3. A. Percentage confluence measured over four days every four hours. B. Area under curve analysis of growth curve analysis. ${ }^{* * * *}, \mathrm{p}<0.0001$. 


\subsection{Changes in Metabolic Activity}

\subsubsection{Culture media $\mathrm{pH}$}

The first and most striking observation noticed about the C57C3 Pten cell line was that the DMEM culture media would begin to change colour when $80 \%$ confluence was reached, while at the same confluence the astrocytes' media had not changed. This suggested that these cells had developed altered glycolytic activity, in the process producing an increased amount of acidic by-products into the media, predominantly lactate. To confirm this hypothesis, all cell lines were left to grow to confluence (approximately 100\%) at which point the media was collected and the $\mathrm{pH}$ measured. DMEM has a $\mathrm{pH}$ buffer range of 7.2-7.8 while exposed to between 5 and $10 \% \mathrm{CO}_{2}$ As a result, under normal conditions, the $\mathrm{pH}$ of the media would not be expected to decrease below this. The astrocytes had a media $\mathrm{pH}$ of 7.54 , while the media of the C57C3 Pten cells decreased to 6.98 at approximately the same confluence (Fig 4.5A).

While comparing the two additional 'classical-like' cell lines, an interesting contrast was seen. Instead of possessing an additive effect on the production of acidic products, two different outcomes were observed. While the Pten + EGFR line showed the same pH as the C57C3 Pten media ( $\mathrm{pH}$ 6.99), the Pten + RAS showed a pH more similar to that of the astrocytes ( $\mathrm{pH} 7.22$ ) (Fig 4.5B). This result implied that while the addition of EGFR VIII has not altered the glycolytic activity, the addition of RAS V12 to the Pten knockout had a normalising effect. 
A
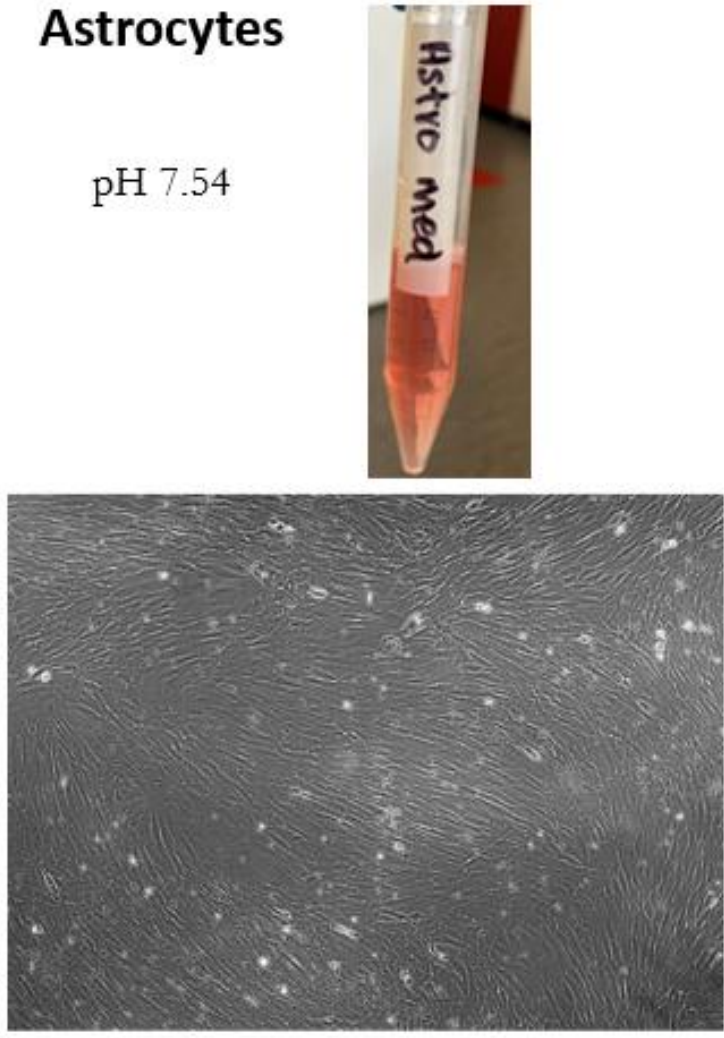

B
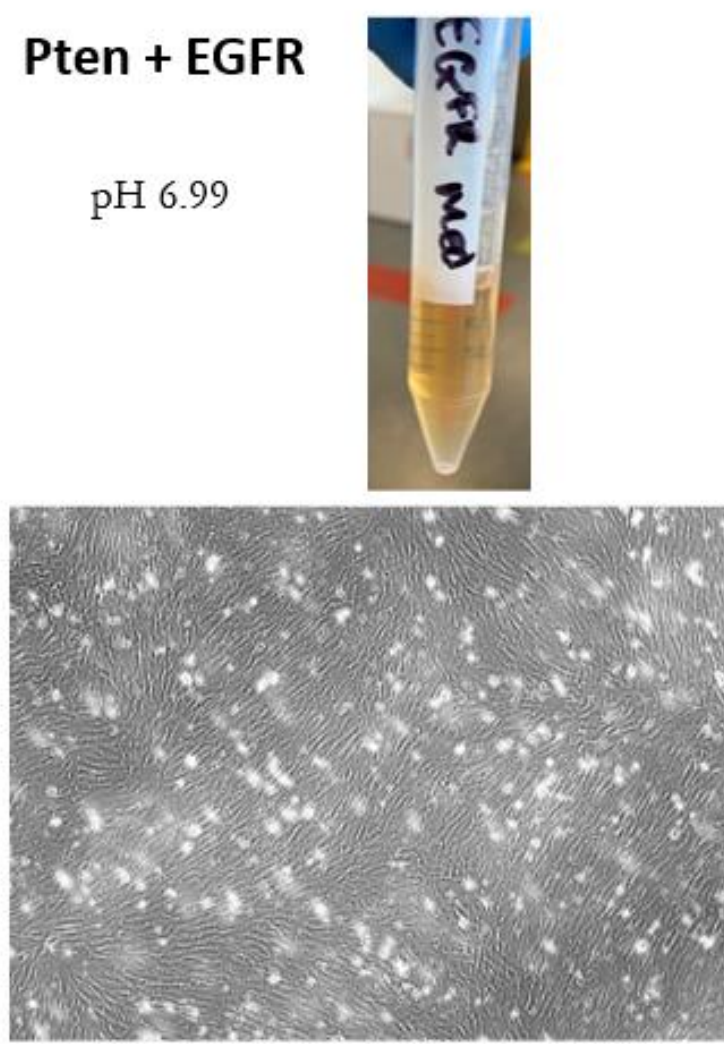

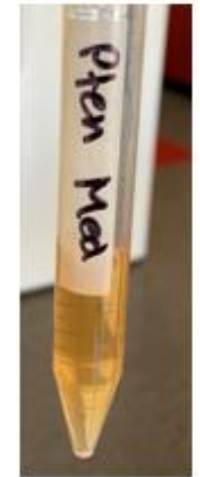

C57C3 Pten
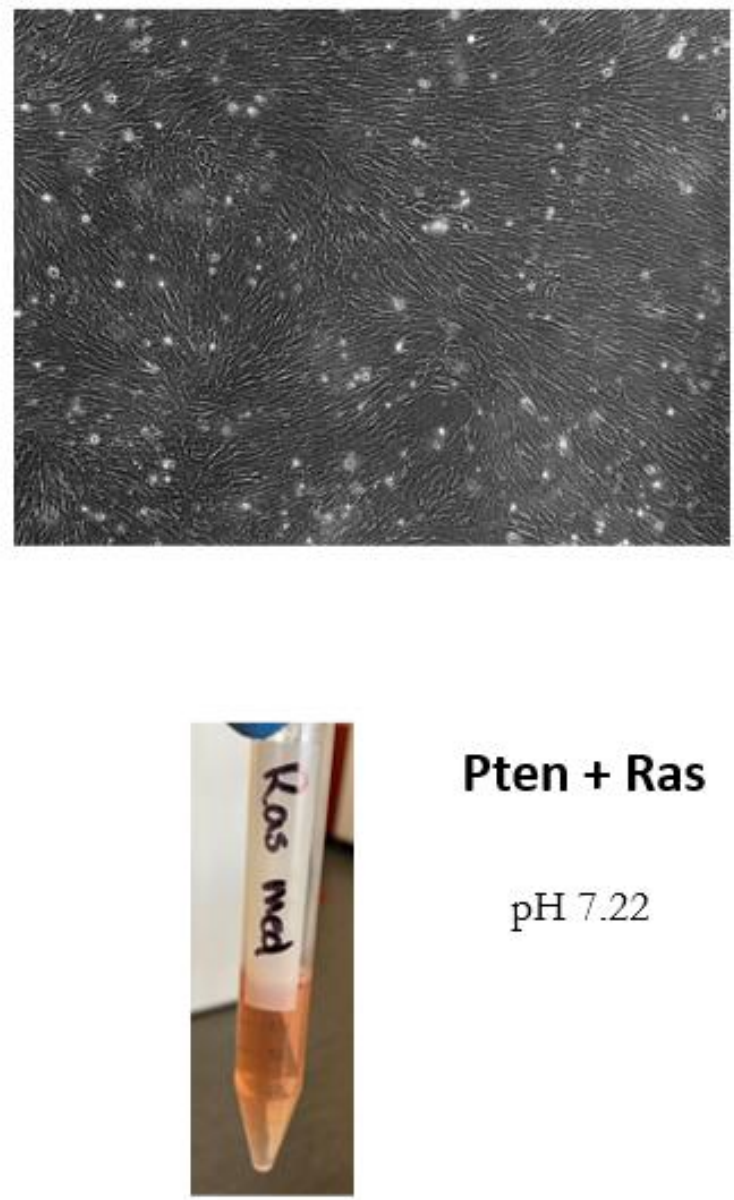

Pten + Ras

$\mathrm{pH} 7.22$

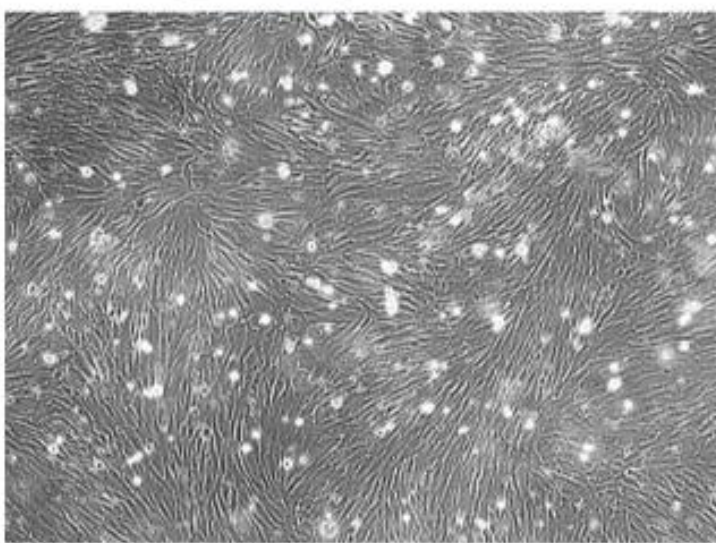


Figure 4.5: $\mathrm{pH}$ Changes in the 'classical-like' culture media $\mathrm{pH}$. Culture media for astrocytes and 'classical-like' cell lines shown with $\mathrm{pH}$ level and microscope image of the corresponding confluence. A. Comparison of astrocytes and C57C3 Pten media pH levels. B. Comparison of Pten + EGFR and Pten + RAS media pH levels.

\subsubsection{Reductive activity of C57C3 Pten and astrocytes}

In order to more accurately characterise the changes in $\mathrm{pH}$, an assay which investigated glycolysis more specifically was developed. The CellTitre MTS assay is used to quantify the concentration of electron carriers which have been produced due to glycolysis and oxidative phosphorylation ${ }^{119}$. This is measured through the changes in absorbance as MTS is reduced to formazan. If there was an increased abundance of electron carriers produced by the cells, there would be a corresponding increase in absorbance at $490 \mathrm{~nm}$. This experiment measured the absorbance at four time points over 96 hours, which complemented the time frame of the proliferation assay. Two aspects of this experiment were analysed: the absorbance itself was used as a semiquantitative indicator of electron carrier production; while the rate of change in absorbance was used as a metric of changes in metabolic activity over time.

The astrocytes and the C57C3 Pten cells showed the same trend of a steady increase in formazan production, and absorbance, until day three and a decline at day four. However, there was a significantly larger peak in absorbance for the C57C3 Pten cells, $t(16)=4.275, \mathrm{p}=0.00174(M=1.188, S E M=0.073, n=3)$ compared with the astrocytes $(M=0.752, S E M=0.028, n=3)$ (Fig 4.6A). The rates were analysed by comparing the AUC for the two curves. This result showed that there was a statistically significant difference in metabolic rate seen between the astrocytes $(M=1.822, S E M=0.1587, n=3)$ compared with the C57C3 Pten $(M=2.842, S E M=0.1741, n=3), t(16)=4.355, \mathrm{p}=0.0005$. 
Absorbance from CellTitre MTS Assay

for Astrocytes Vs C57C3 Pten

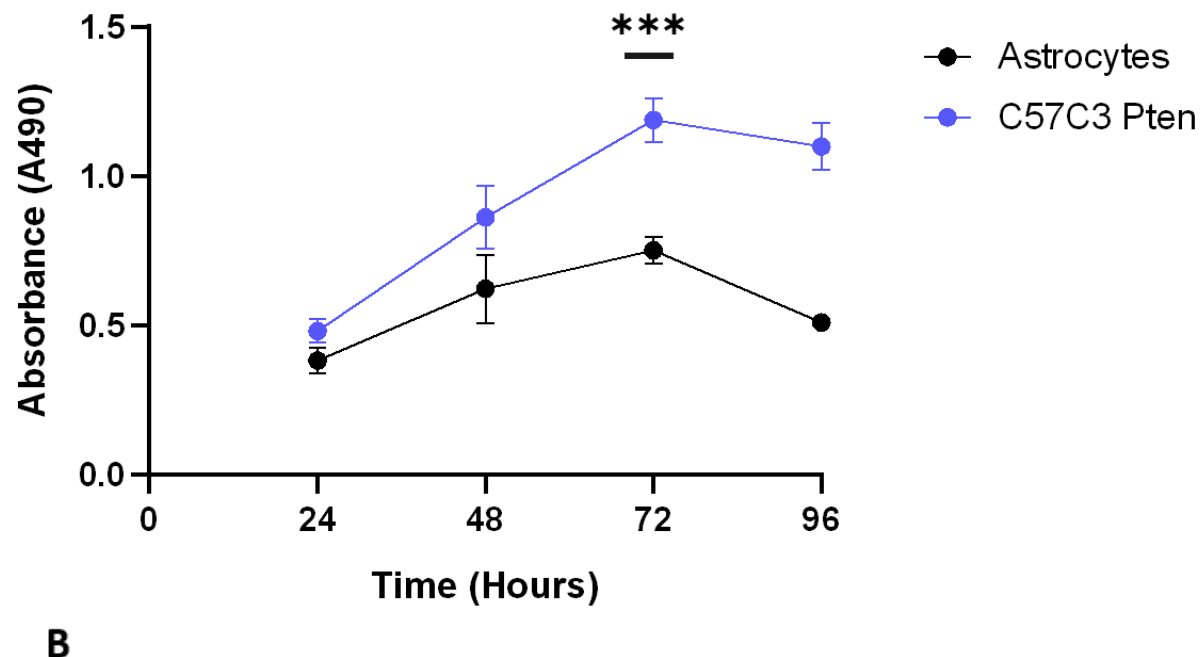

AUC of MTS Assay Absorbance for Astrocytes Vs C57C3 Pten

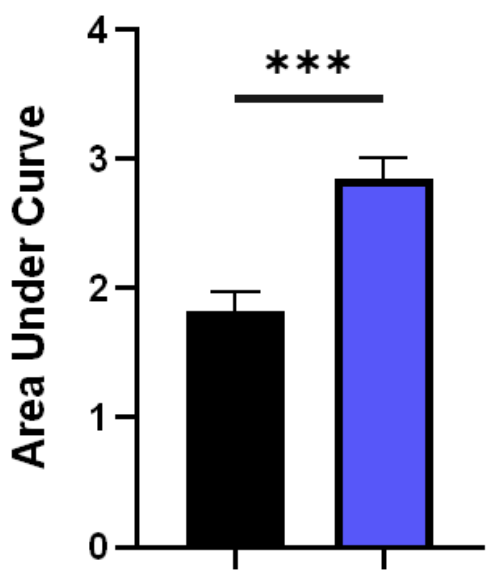

- Astrocytes

口 C57C3 Pten

Cell Lines

Figure 4.6: Comparison of MTS assay in astrocytes and C57C3 Pten cells. MTS assay results for astrocytes (black) and C57C3 Pten cells (blue). Absorbance measured every 24 hours. Error bars represent SEM and n=3. A. Absorbance (A490) of MTS assay over a four-day period. At day three, ${ }^{* * *}, \mathrm{p}=0.00174$. B. Area under curve analysis for rate of MTS absorbance change over four days, ${ }^{* * *}, \mathrm{p}=0.0005$. 


\subsubsection{Reductive activity of the 'classical-like' GEM-CLeMs}

The MTS assay was conducted on both the RAS V12 and EGFRVIII lines, in order to assess whether the differences in acidity are coupled with a concomitant change in electron carrier production. The Pten + RAS line showed a very similar trend to the astrocytes and C57C3 Pten cells, however, the absorbance did not reach as high a peak by day three $(M=1.006, S E M=0.044, n=3)$ (Fig 4.7A). The C57C3 Pten and Pten + RAS lines showed a consistent gradient in absorbance change between days one and three; this indicated steady period of electron carrier production was between these days. The Pten + EGFR cells, however, showed a slightly altered trend. The EGFR line showed the greatest gradient between days two and three. This implied that, unlike the other lines, the Pten + EGFR cells increased the rate of electron carrier production between days two and three. Additionally, the peak in absorbance in the Pten + EGFR wells was seen on day four $(M=1.185, S E M=0.066, n=3)$, rather than day three (Fig 4.7A). In order to quantify any differences in the rate of electron carrier production between these cell lines, the AUC was compared. No statistical difference was seen between the three 'classical-like' lines in terms of production of electron carriers, $\mathrm{F}(2,20)=1.672$, $\mathrm{p}=0.2131$ (Fig 4.7B). 


\section{Absorbance from CellTitre MTS Assay for "Classical-Like" Cell Lines}

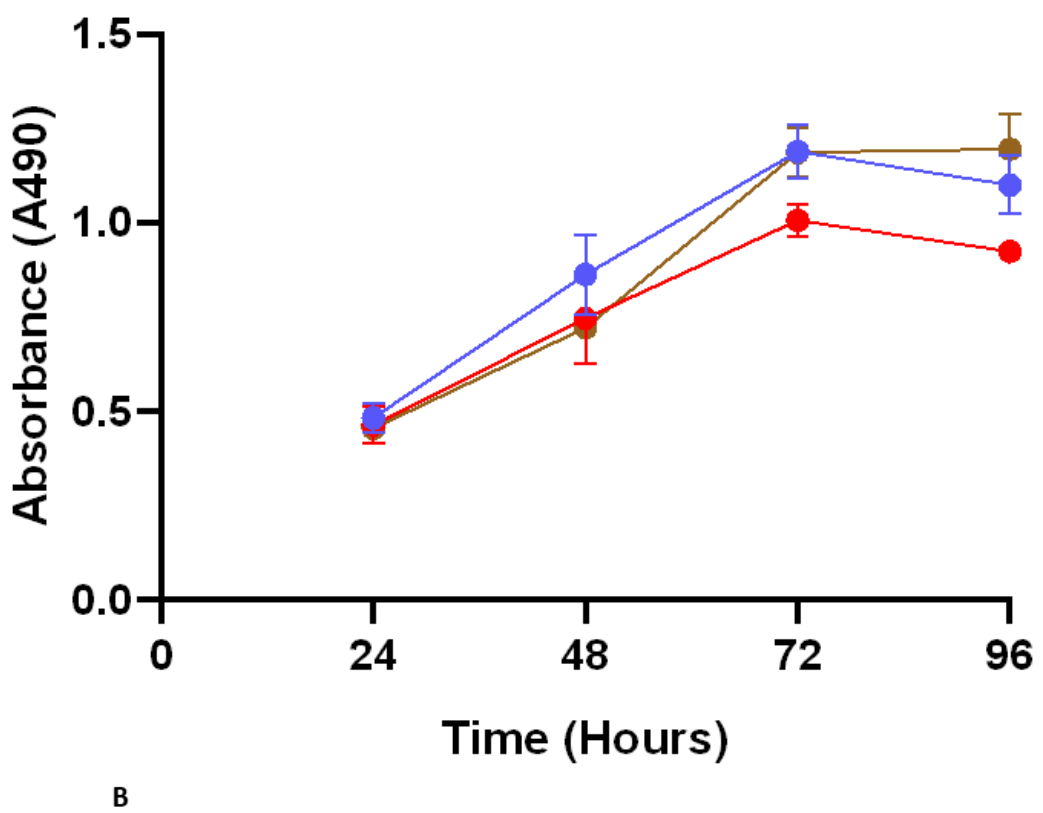

AUC of MTS Assay Absorbance
for "Classical-Like" Cell Lines

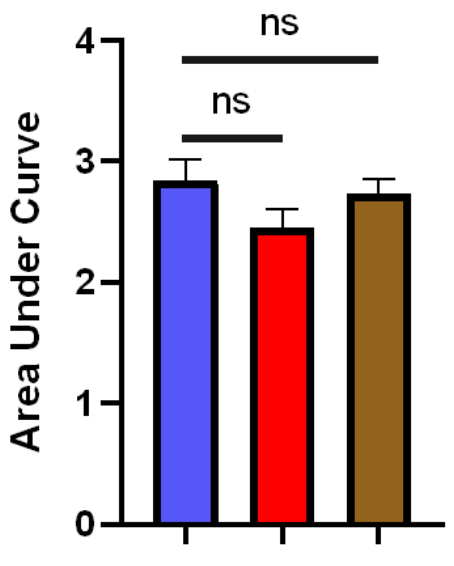

ㅁ C57C3 Pten

ㅁ Pten + RAS

D Pten + EGFR

Cell Lines

Figure 4.7: Comparison of MTS assay in 'classical-like' GEM-CLeMs. MTS assay results for C57C3 Pten (blue), Pten + RAS (red), and Pten + EGFR (brown). Absorbance measured every 24 hours. Error bars represent SEM and $n=3$. A. Absorbance (A490) of MTS assay over a four-day period. B. Area under curve analysis for rate of MTS absorbance change over four days. ns (C57C3 Pten and Pten + RAS), $\mathrm{p}=0.1578$. ns (C57C3 Pten and Pten + EGFR), $\mathrm{p}=0.8841$. 


\subsection{Characterising Migration of the Cell Lines}

\subsubsection{Cell migration and invasion}

The classical phenotype of GBM is associated with de novo tumours, which are typically more lethal than secondary cancers. An important reason for this is the invasive nature of the tumours. Classical and mesenchymal GBM tend to have much more rapid tumour growth which infiltrates into the healthy parenchyma when compared with LGG and secondary cancers ${ }^{25}$. Because of this, the migrative capacity of the cell lines was measured. This was conducted using the Incucyte ${ }^{\circledR}$ 'wound healing' function. Using a tool called the 'Wound Maker 96', uniform wounds were made to the confluent layer of cells in a 96-well plate. The Incucyte ${ }^{\circledR}$ then recorded a series of images to identify when the cells migrated back into the wound and quantified the rate at which this happened. The metric chosen for this was 'wound density' as a percentage. The Incucyte ${ }^{\circledR}$ calculated the number of cells which had migrated into the wound, rather than the confluence which solely accounts for area. The rate at which the cell lines migrated indicated whether the cell lines had acquired an invasive phenotype.

\subsubsection{Optimisation}

The primary experiments using the wound healing function of the Incucyte ${ }^{\circledR}$ required optimising. The initial experimental design involved plating the cell lines at a high confluence (approximately 90\%) and allowing the cells to adhere. However, the results from this experiment showed that the C57C3 Pten lines were overconfluent at the time of introducing the wound. This result led to a change in the confluence that the cells were plated at in this assay. Cells were plated at $40 \%$ and left to adhere overnight. As a result, the wells showed approximately $80 \%$ confluence at the time the wound was made, and the results were much more consistent between replicates. 


\subsubsection{Comparing C57C3 Pten and astrocyte migrative ability}

Migration was first compared between astrocytes and the C57C3 Pten cells. Over a period of 24 hours, photos were taken every hour and wound density was measured. An example of the images assessed is shown in figure 4.8. After 24 hours, the C57C3 Pten cells had a mean wound density of $68.57 \%(S E M=6.762, n=3)$ compared with the astrocytes mean of $40.43 \%(S E M=6.969, n=3)$ (Fig 4.9A). The AUC was measured to compare the rate of migration between the cell lines. There was a statistically significant difference in the rate of migration between the C57C3 Pten cells, $t(6)=7.429, \mathrm{p}=0.0003(M=778.8$, $S E M=22.14)$ and the astrocytes $(M=486.9, S E M=32.46)$ (Fig 4.9B). This result showed that through the introduction of the Pten indel, there was an increase in the rate at which the C57C3 Pten can migrate in vitro.
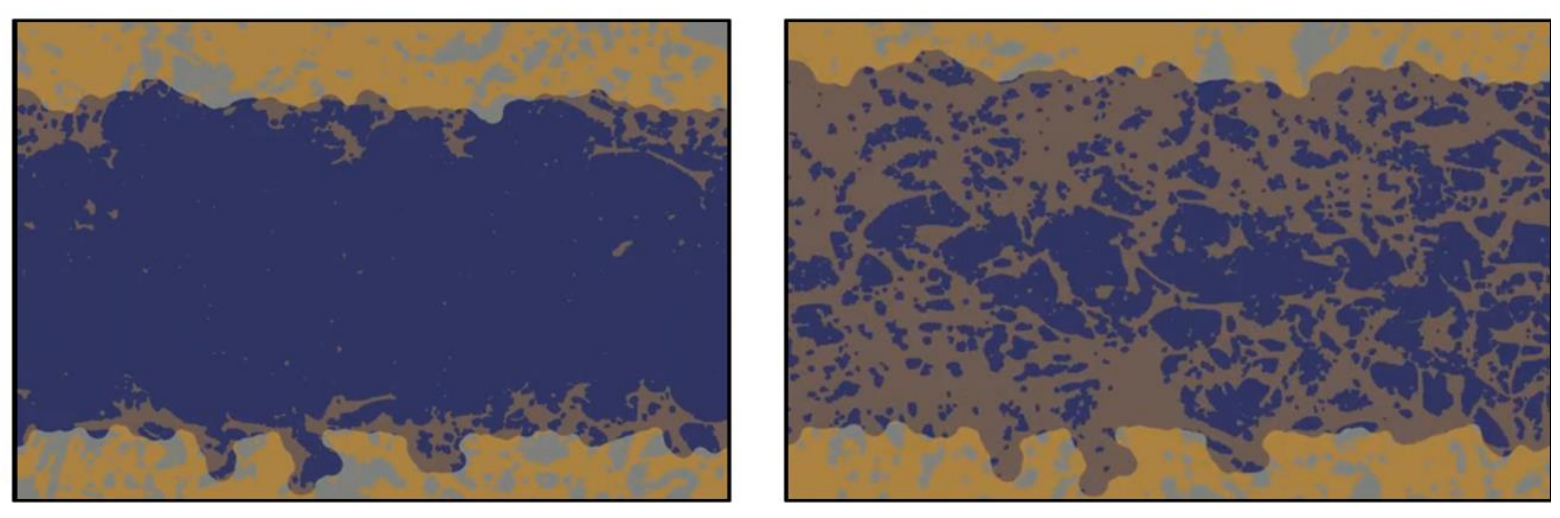

Figure 4.8: Example of the migration observed in the wound healing assay. Incucyte ${ }^{\circledR}$ images of wound healing assay. Wound created by WoundMaker96 (blue) and Pten + RAS cells (gold). Left image: taken at four hours post-wound. Right image: 20 hours post-wound 


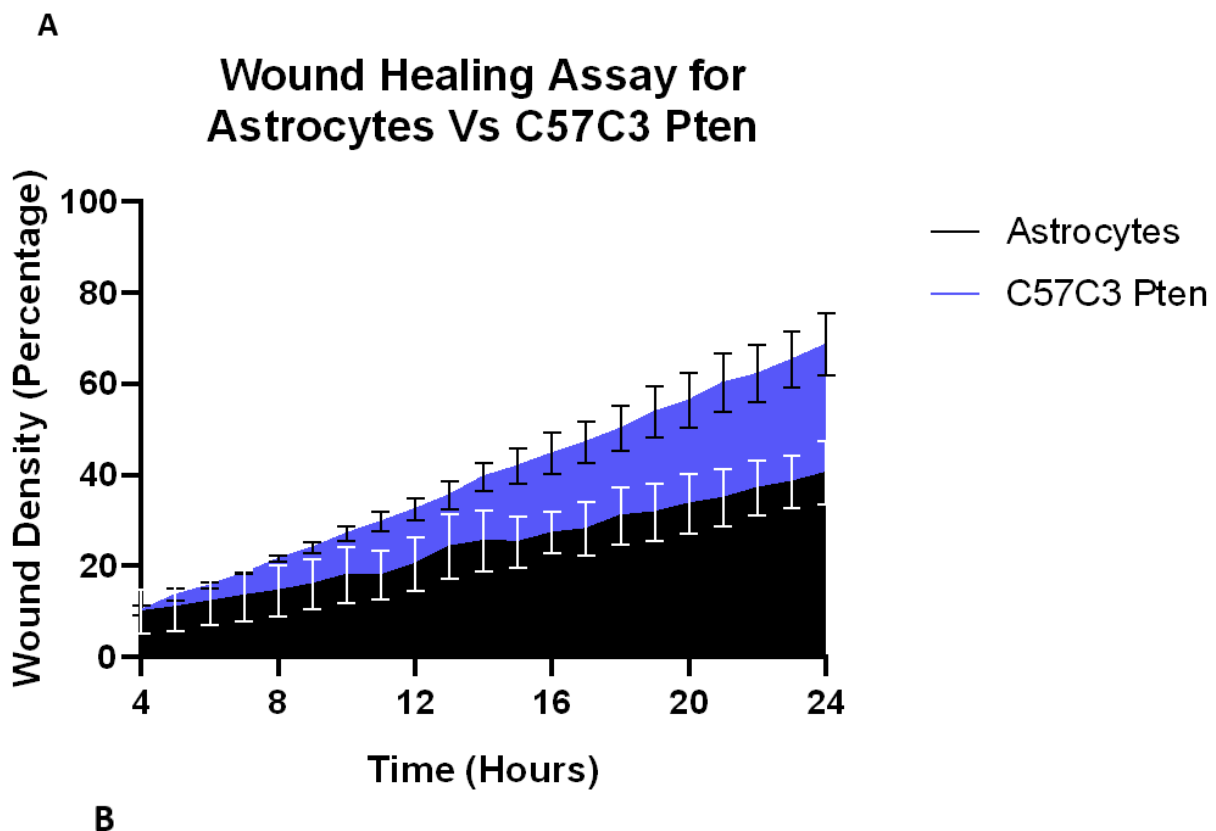

B

AUC of Wound Healing for Astrocytes Vs C57C3 Pten

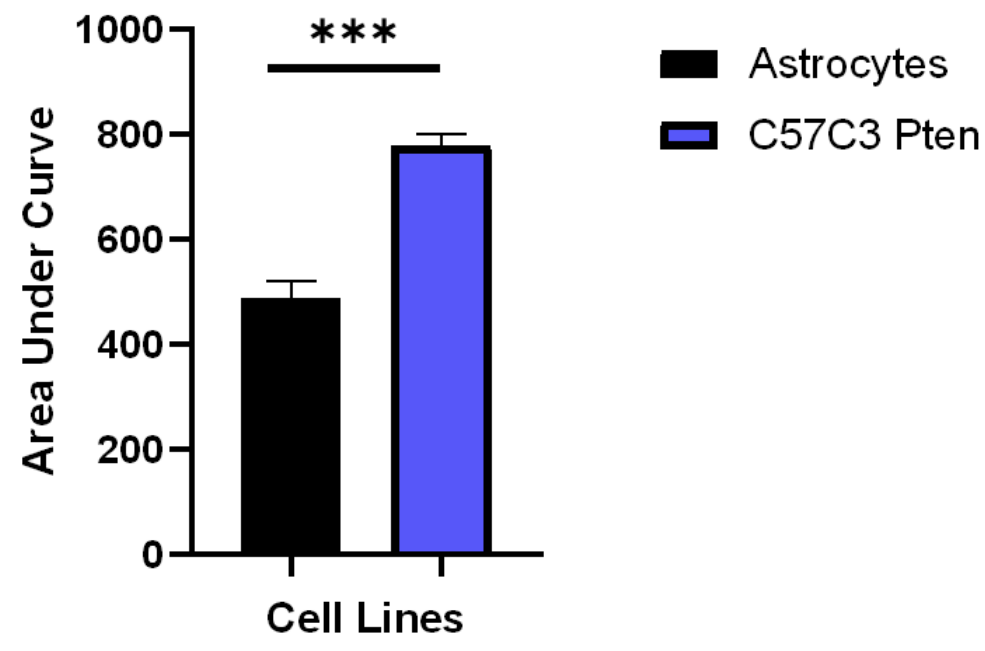

Figure 4.9: Wound healing assay for astrocytes and C57C3 Pten cells. Wound healing assay data for the astrocytes (black) and C57C3 Pten cells (blue), collected from the Incucyte ${ }^{\circledR}$ over 24 hours (hours four to 24 shown). Error bars represent SEM and $\mathrm{n}=3$. A. Wound density (percentage) over time. B. Area under curve analysis of wound density, $* * *, p=0.0003$. 
Following the comparison of the C57C3 Pten line to the astrocytes, the next aim was to assess any additive or decreased migrative ability through the addition of RAS V12. After 24 hours, it was seen that more Pten + RAS cells had migrated into the wound area $(M=81.77 \%, S E M=3.230, n=3)$ compared with C57C3 Pten $(M=68.57 \%, S E M=6.762$, $n=3$ ) (Fig 4.10A). Additionally, the AUC comparing the rate of migration showed a statistically significant difference, $t(6)=2.886, \mathrm{p}=0.0279$ (Fig 4.10B). This result indicated that the addition of RAS V12 on top of an indel in Pten resulted in an additive increase in the cell line’s migrative ability. 
A Wound Healing Assay for C57C3 Pten Vs Pten + RAS

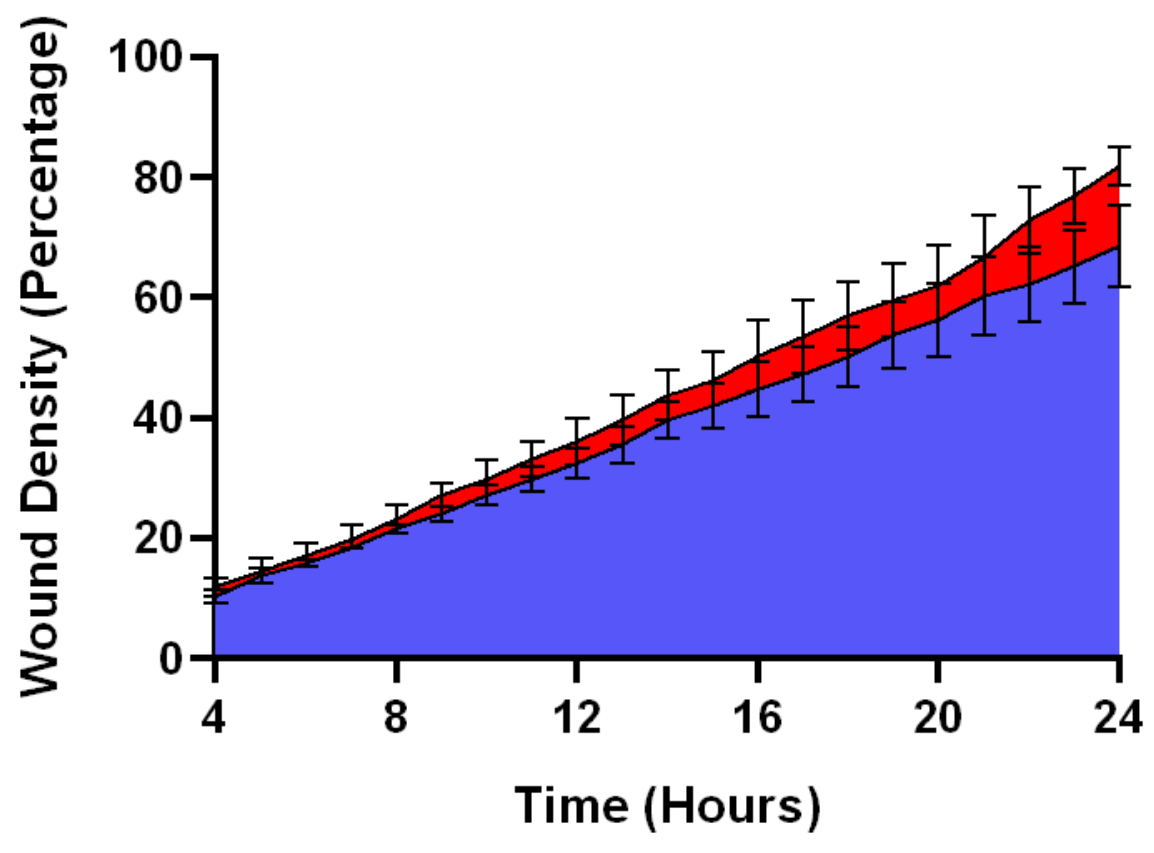

B

AUC of Wound Healing Assay
for C57C3 Pten Vs Pten + RAS

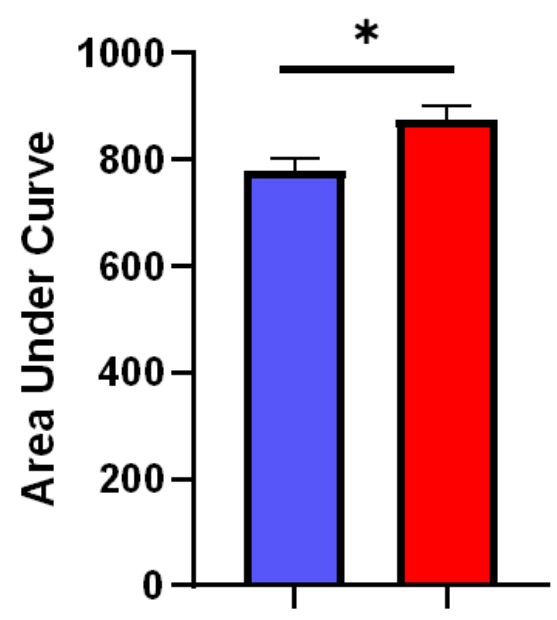

口 C57C3 Pten

口 Pten + RAS

Cell Lines

Figure 4.10: Wound healing assay for C57C3 Pten and Pten + RAS cells. Wound healing assay data for the C57C3 Pten (blue) and Pten + RAS cells (red), collected from the Incucyte ${ }^{\circledR}$ over 24 hours (hours four to 24 shown). Error bars represent SEM and $\mathrm{n}=3$. A. Wound density (percentage) over time. B. Area under curve analysis of wound density. *, $\mathrm{p}=0.0279$. 


\subsection{Clonogenic Potential of Cell Lines}

\subsubsection{Clonogenic assay}

The clonogenic assay, also known as a colony forming assay, is a type of cancer stem cell characterisation. This assay involved plating the cells at a very low density and assessing their ability to develop into colonies from a single cell. The characteristics involved are a) how efficiently an individual cell can develop into a colony, and b) the speed at which these individual colonies develop. Since the classical subtype of GBM tend to possess more proliferation related mutations $22,25,27$, it was hypothesised that the 'classicallike' cells would have an increased division speed. The strategy developed to assess the differences seen in this assay involved two components a) a quantitative comparison of the area the colonies occupy of each well (Fig 4.11), and b) a qualitative comparison of the density of each colony. Colony density was considered an indication of whether a cell is predisposed to proliferation or migration, as a cell cannot simultaneously achieve both.

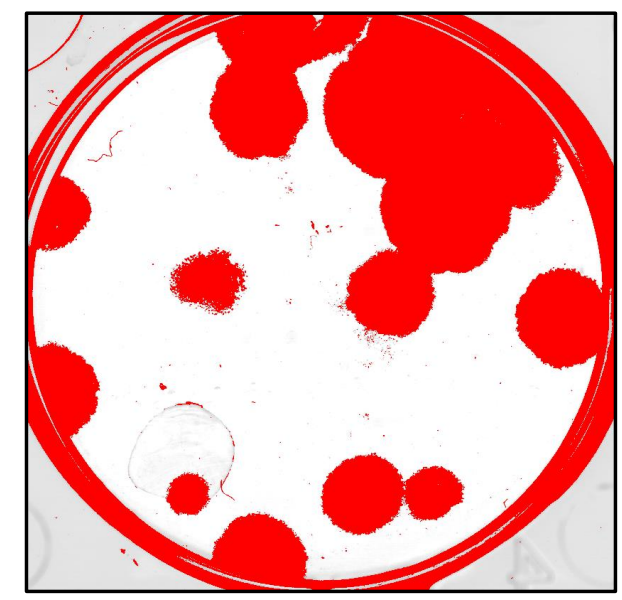

Figure 4.11: Example of the clonogenic assay analysis. C57C3 Pten clonogenic assay, 15 cell seeding density. Threshold produced with ImageJ. Area occupied (Red) represents the colonies developed over two weeks. 


\subsubsection{Comparing the C57C3 Pten and astrocyte clonogenic ability}

Fifteen, 30 and 60 cells were plated in each well of a six-well plate and left to grow two weeks. The resulting data showed that at all seeding densities the C57C3 Pten cell line covered a greater relative area of the well than the astrocytes; 15 cells, $t(2)=17.12, p=0.0034$; 30 cells, $t(2)=10.67, p=0.0087 ; 60$ cells, $t(2)=11.39, p=0.0076$ (Fig 4.12B). The major observation when visually comparing the plates was that the C57C3 Pten line produced much larger colonies over the same period than the astrocytes. The size of the colonies made it difficult to assess whether the Pten cells had an increased or decreased survival compared with the astrocytes, as many of the colonies overlap. Both cell lines produced a mix of dense and spread-out colonies (Fig 4.12A). This result showed that within the cells plated, the C57C3 Pten and astrocyte cell lines both had variation in the degree of proliferation. This was seen through the even mixture of dense and spread out colonies. 
A

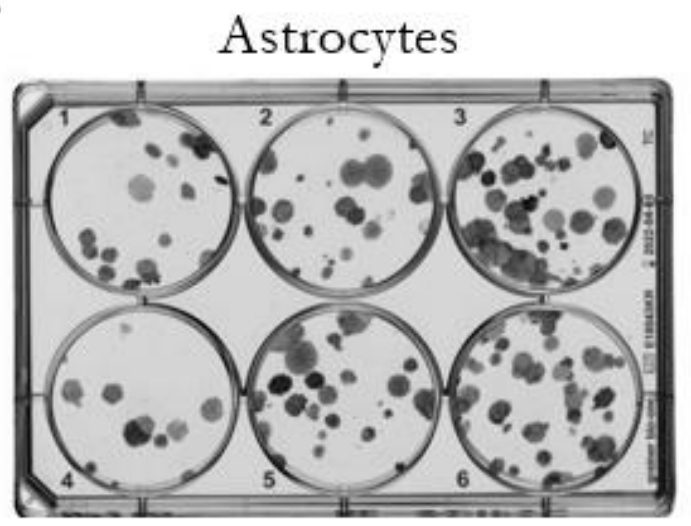

15 Cells $\quad 30$ Cells 60 Cells
C57C3 Pten

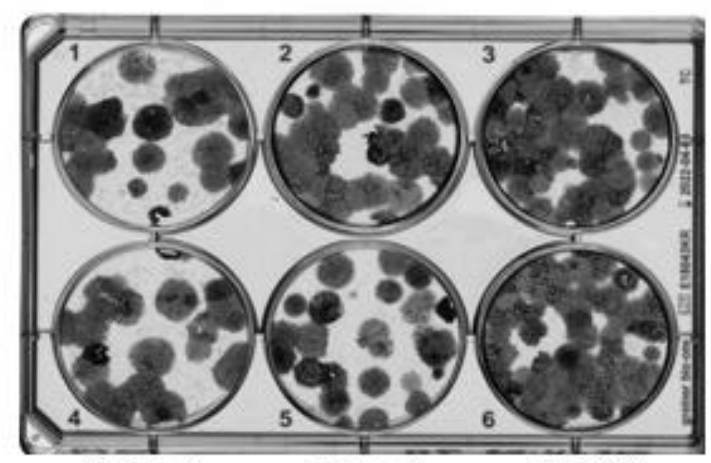

15 Cells 30 Cells 60 Cells

\section{B \\ Clonogenic Assay for Astrocytes Vs C57C3 Pten}

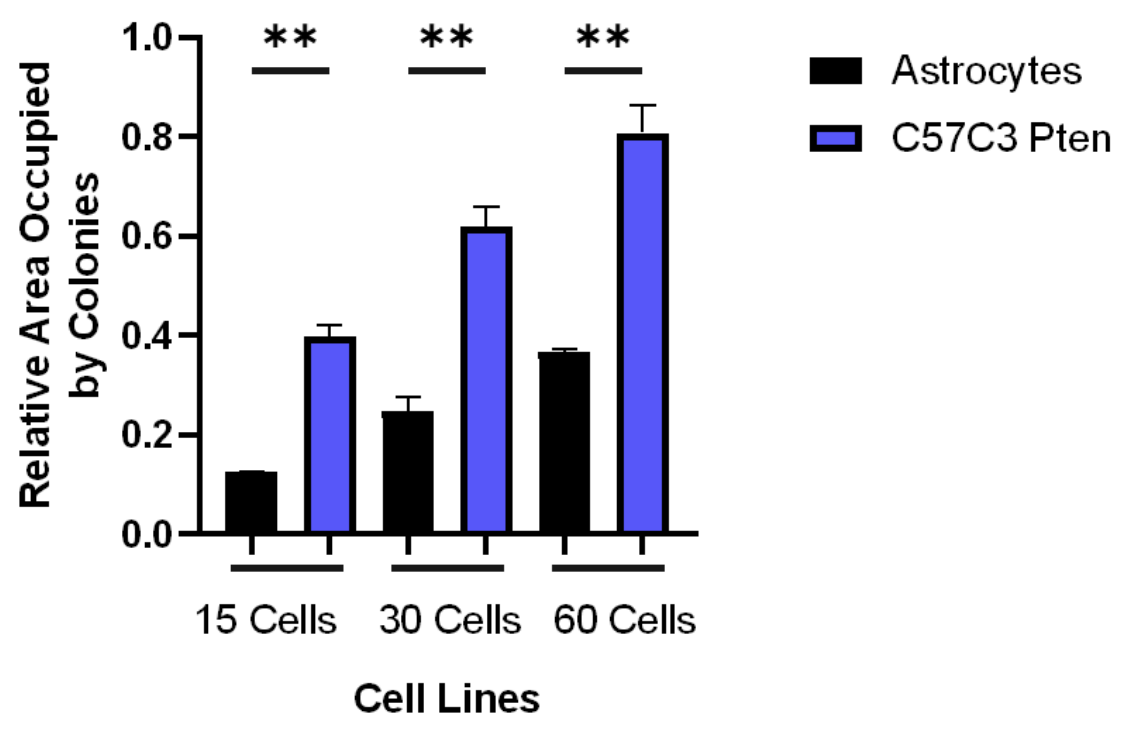

Figure 4.12: Clonogenic assay results for astrocytes and C57C3 Pten cells. A. Methylene blue stained colonies after two weeks of clonogenic assay, shown as representation of data. Wells shown as technical duplicates. Columns represent the number of cells seeded at the start of the assay. $\mathbf{B}$. Relative area occupied by the colonies for astrocytes (black) and C57C3 Pten cell lines (blue). Data shown for 15, 30 and 60 cell seeding density. Error bars represent SEM, n= 3 . 


\subsubsection{Comparing the 'classical-like' GEM-CLeMs clonogenic ability}

The next aim was to assess whether the addition of the oncogenes led to an additive clonogenicity in Pten + RAS and Pten + EGFR cell lines. The assay showed that the C57C3 Pten cells covered a greater portion of the plate at the 15 cell density when compared with both Pten + RAS and Pten + EGFR cells; $F(2,3)=10.92, p=0.0420$. Post-hoc analysis indicated that both the mean relative area covered for the Pten + RAS $(M=0.3024$, $S E M=0.121, n=3)$ and the Pten + EGFR $(M=0.3057, S E M=0.0715, n=3)$ were significantly less than the mean relative area of the C57C3 Pten line $(M=0.3995$, $S E M=0.0475, n=3)$. A significant difference was also detected when 30 cells were plated, $\mathrm{F}(2,3)=13.96, \mathrm{p}=0.0302$. Post-hoc analysis indicated that there was a significant difference between the Pten + RAS $(M=0.4409, S E M=0.1355, n=3)$ and the C57C3 Pten lines $(M=0.6197, S E M=0.039, n=3)$. There was no significant difference seen between the C57C3 Pten and Pten + EGFR lines $(M=0.4963$, SEM=0.0815, $n=3)$. Finally, when comparing the 60 cell seeding density, no significant difference was seen between the cell lines, $\mathrm{F}(2,3)=7.273, \mathrm{p}=0.0707$ (Fig 4.13B).

Three major differences were seen in the comparison of the 'classical-like' GEMCLeMs. Firstly, the C57C3 Pten had larger colonies than the other 'classical-like' lines. Secondly, the Pten + EGFR wells showed a higher proportion of dense colonies; this indicated that the Pten + EGFR cell line potentially showed more of a tendency towards proliferation than migration. And finally, the Pten + RAS colonies appeared to be on average the least dense, which implied that of the three 'classical-like' cells this line was the most migratory (Fig 4.13A). Overall, this assay showed that the three cell lines showed different clonogenic abilities, despite sharing the same Pten mutation. 
A

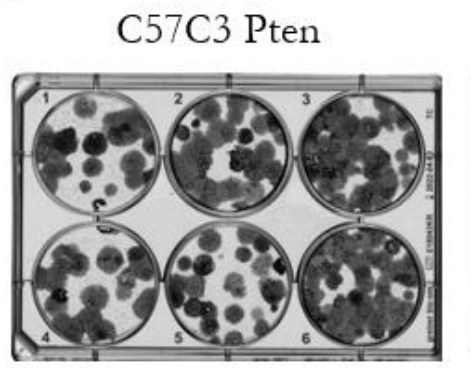

15 Cells

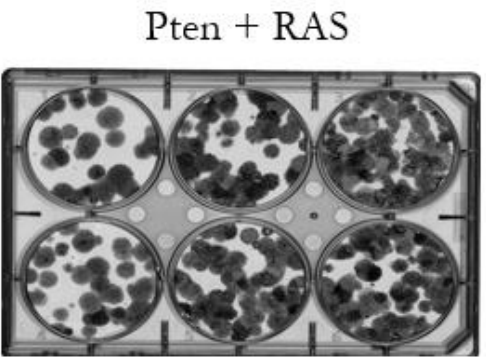

15 Cells 30 Cells 60 Cells

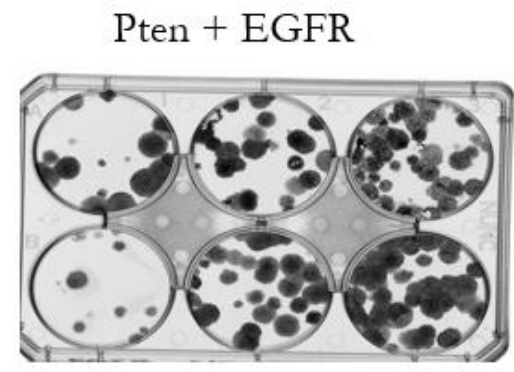

15 Cells 30 Cells 60 Cells

B

\section{Clonogenic Assay for "Classical-Like" Cell Lines}

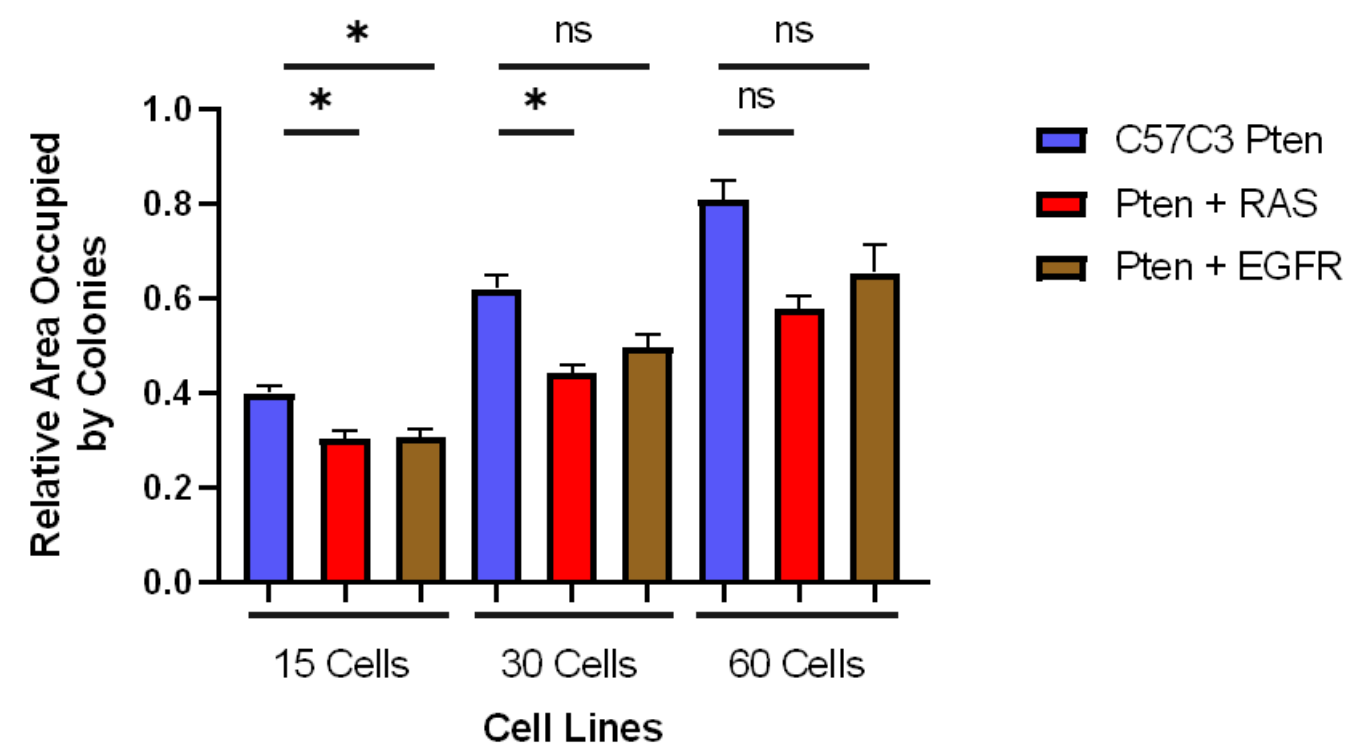

Figure 4.13: Clonogenic assay results for 'classical-like' GEM-CLeMs. A. Methylene blue stained colonies after two weeks of clonogenic assay, shown as representation of data. Wells shown as technical duplicates. Columns represent the number of cells seeded at the start of the assay. B. Relative area occupied by the colonies for C57C3 Pten (blue), Pten + RAS (red) and Pten + EGFR cell lines (brown). Data shown for 15, 30 and 60 cell seeding density. Error bars represent SEM, $n=3$. 


\subsection{Discussion}

This chapter characterised and compared three different cell lines: C57C3 Pten, Pten + EGFR, and Pten + RAS. The assays were chosen to investigate an array of both general cancer cell phenotypes and specific classical GBM subtype phenotypes. Proliferation was used as the major comparator from which alterations in other aspects could be seen, such as migration, metabolism and clonogenicity. Unfortunately, due to a technical issue with the Incucyte ${ }^{\circledR}$ towards the end of the project, proliferation data from the RAS and EGFR lines could not be generated. Because of this, a comparison cannot be made of the baseline proliferation rate between the C57C3 Pten cells and the RAS and EGFR lines. Additionally, no conclusive data had been generated on the Pten + EGFR wound healing assay prior to the demise of the Incucyte ${ }^{\circledR}$.

As all the 'classical-like' GEM-CLeMs contained the same Pten mutation, it was important that the C57C3 Pten cell line was fully characterised. This characterisation showed how the cells had been altered from the original astrocytes. Furthermore, the data for the C57C3 Pten cells showed how the key oncogenes impacted the specific classical subtype phenotype. The first result seen in comparing the astrocytes with C57C3 Pten cells was that the loss of Pten activity had increased the proliferation rate. This was expected as the literature surrounding PTEN deletions in cancer all corroborate the proteins role in driving tumour progression 28,30,31,120-123. The results showed high levels of statistical significance in the proliferation comparison of the C57C3 Pten and the astrocytes. This was seen because the high number of technical replicates and timepoints used in the experimental design. However, I believe that while this result was significant, this is not the most biologically important result that was seen in the characterisation of the C57C3 Pten cell line.

Through normalisation of the C57C3 Pten data to the astrocyte data, the greatest changes in the phenotypes can be seen. These comparisons showed that clonogenicity was the characteristic most altered by the reduced Pten activity. While the results for C57C3 Pten showed a 50\% increase in the MTS assay and migration over the astrocytes, the colony formation was 150\% higher (Fig 4.14). This indicated that despite all the other cancer 
phenotypes increasing with the Pten loss, the most significant change was in each individual cell's ability to generate a colony. The method chosen to analyse the clonogenic assay was to compare the relative area which the colonies occupied. This investigated not only the seeding efficiency, but also the proliferation rate at a very low confluence. The proliferation and MTS assays each began with a normal seeding density (5\%), and when measuring migration, the wound was introduced at $80 \%$ confluence. Hence these assays were performed under normal conditions where growth factors are abundant in the media. In contrast, the clonogenic assay had a seeding density as low as 15 cells per well, which is approximately 20,000x lower than the normal seeding density ${ }^{124}$. This meant that under stress conditions and extreme low confluence, the C57C3 Pten line has a significantly increased clonal growth rate. This result is potentially important with regards to in vivo tumorigenicity. The astrocytes have previously been intracranially transplanted into mice and did not develop into a tumour (McConnell, unpublished data). The C57C3 Pten cell line's increased ability to grow rapidly under stress, indicated that after transplantation these cells may be more likely to grow in vivo. 


\section{Normalised Data for Astrocytes Vs C57C3 Pten}

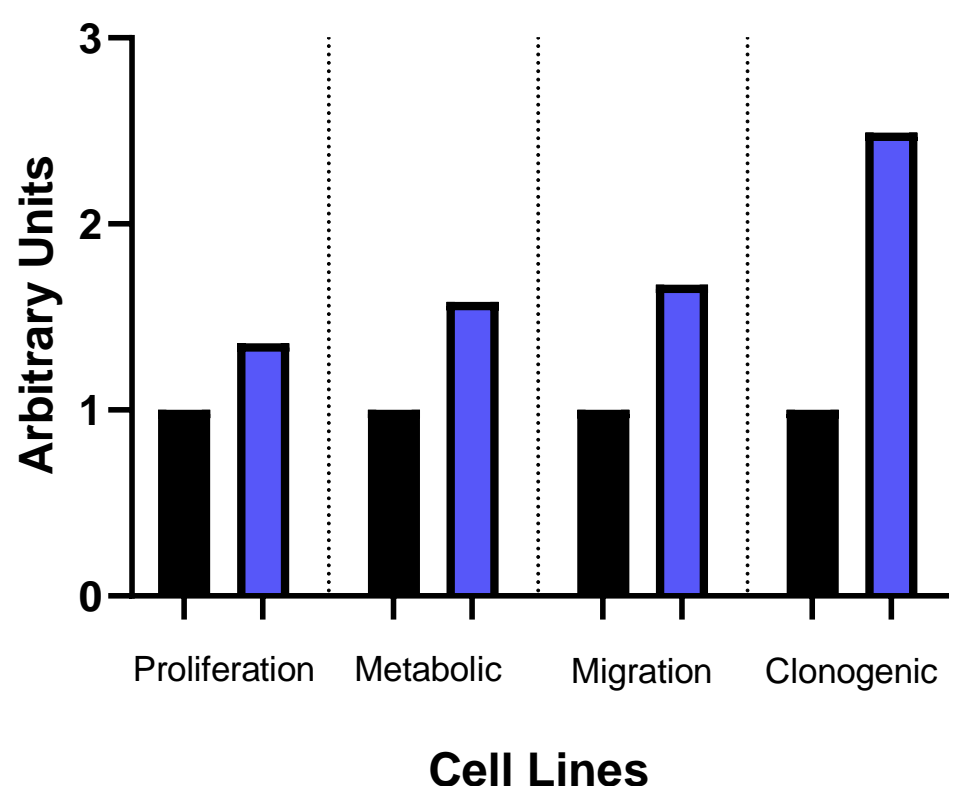

Figure 4.14: Normalised data comparing the astrocytes and C57C3 Pten assays. Data gathered from the proliferation assay (4.4.2), MTS/metabolic assay (4.5.2), wound healing/migration assay (4.6.3), and clonogenic assays (4.7.2) shown when normalised to the results from the astrocytes.

In the wound healing assay, an alteration to the well-established protocol was required. Initially, when plated at 90\% confluence, a larger than intended wound was introduced to the C57C3 Pten cells, and not the astrocytes which were plated at the same density. Visually comparing the two cell lines at confluence, differences in the morphology were seen. While it is normal for the astrocytes to pack together closely, the individual cells can be made out under the microscope. However, the C57C3 Pten cells show much more elongation and pack together more tightly. I believe that this change in morphology at confluence results in stronger physical connections with neighbouring cells; as a result, this dislodged more cells than the Wound Maker and Incucyte ${ }^{\circledR}$ software were designed to induce and analyse. 
A major finding from these results was that the Pten + RAS cell line demonstrated an increased migration. This was seen through the increased rate at which the cells were able to migrate back into the wound, as seen on the Incucyte ${ }^{\circledR}$. This result showed that, in addition to the increased proliferation that the Pten mutation has provided, the RAS V12 oncogene resulted in a much more migratory phenotype. This observation was consistent with the clonogenic assay, where the Pten + RAS cells showed the most spread-out colonies. While these colonies did not cover as much area in the well as the Pten knockout line, this migration was noticeable as the colonies were consistently less dense. This was important in the aim of developing a 'classical-like' cell line as the key feature of these tumours is not only the speed of growth but also the invasion into the healthy surrounding tissue.

RAS is an extremely frequent mutation over a wide range of cancer types; a mutation in the gene is present in approximately $20 \%$ of all tumours 125,126 . RAS is a pivotal protein in proliferative cell signalling. The protein is responsible for directly initiating the MAPK phosphorylation cascade, and as a result is crucial for the numerous downstream effects. In addition to this, as a GTPase, RAS is involved in many other cell signalling pathways. For example, RAS is directly linked to the AKT/mTOR pathway as it is allosterically involved in phosphorylation of PI3K - the inhibitory target of PTEN. In this study, the introduction of RAS V12 resulted in a more migratory phenotype. The concept that a RAS mutation would result in a more invasive tumour has been seen in numerous cancers ${ }^{34,126}$. In the skin cancer, cutaneous squamous cell carcinoma, the presence of a constitutively activated RAS resulted in an increase of laminin-332 through TGF- $\beta$ signalling. This resulted in a significant increase in tumour migration and invasion ${ }^{127}$. In lung cancer, following ionising radiation, radiation-induced tumour invasion was seen only in RAS mutant samples. This was believed to occur through the Cathepsin L/CUX 1 pathway ${ }^{128}$. From this literature, it appears appropriate that the introduction of RAS V12 to $\mathrm{C} 57 \mathrm{C} 3$ Pten has the effect of increasing cellular migration.

However, there is an important limiting caveat of this GEM-CLeM; despite having very similar downstream effects to EGFR, RAS mutations are extremely uncommon in GBM. In TCGA data, only 3 of 248 tumour samples $(1.2 \%)$ contained a mutant RAS 
protein. In comparison, EGFR mutations occurred in 139 of the GBM tumours (56\%) and 76 had loss-of-function mutations in PTEN (31\%) (Fig 4.15). It is even more striking that no tumour contained EGFR/RAS or PTEN/Ras ${ }^{27}$. However, while this model may not be as clinically relevant as the Pten + EGFR cell line (a pair of mutations seen in 50\% of PTEN altered GBM), this combination of mutations still has the potential to improve on the current GBM models. Currently it is estimated that $91 \%$ of brain related mouse models use cell line transplantation as opposed to PDX and GEM models ${ }^{67}$. GL261 is one of a few models available which can be transplanted into immune competent mice, yet it does not resemble that pathology of a GBM ${ }^{129}$. From the results I have generated, the Pten + RAS cell line shows potential to model an invasive GBM more accurately than GL261.

Unfortunately, due to the impact of COVID-19, the Incucyte ${ }^{\circledR}$ could not be repaired by the appropriate technicians. This meant that the Pten + EGFR cell line was not able to be fully characterised and compared alongside the other 'classical-like' models. The remaining proliferation and wound healing data will be collected when the Incucyte ${ }^{\circledR}$ is functional again. However, from the data collected, this model poses itself as the most clinically relevant to the classical subtype. Despite the importance of heterogeneity in GBM, the reason for the name "classical" is due to the comparatively high prevalence of alterations to EGFR. As mentioned previously, EGFR mutations are seen in approximately half of the TCGA GBM samples. In addition to this, PTEN and EGFR co-mutations are the most prevalent of patterns seen in primary GBM (Fig 4.15). 
PTEN

EGFR
$31 \%$

$56 \%$

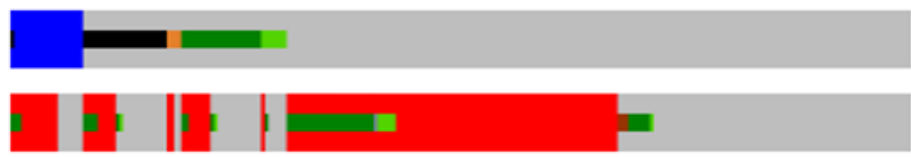

Amplification (putative driver)

Missense Mutation (putative driver)

Splice Mutation (putative driver)
Deep Deletion (putative driver)

Missense Mutation (unknown significance)

- Truncating Mutation (putative driver)

Figure 4.15: TCGA (2013) data on PTEN and EGFR mutations frequency in GBM. Bar represents each of the 248 samples in the TCGA genomic study. Percentages refer to the proportion of samples which contain alterations in the gene. Data from cBioPortal

This model has the potential to improve the biological understanding and the potential treatments of GBM. Interesting trends can be seen comparing the overall survival of patients with EGFR and PTEN mutations in their tumours. In the 2013 TCGA data, the median survival for patients with PTEN mutations is lower than when EGFR alterations alone are present. However, the worst prognosis is seen in patients with mutations in both genes. Additionally, despite a lower median, PTEN-only patients show a greater survival at what is known as the 'tail', as a portion of patients with these tumours are seen to survive past 35 months (Fig 4.16). This trend implies that while PTEN only tumours may progress faster than EGFR only tumours, the co-occurrence of PTEN and EGFR mutations results in an overall worse prognosis with less survival after 15-months 27. 


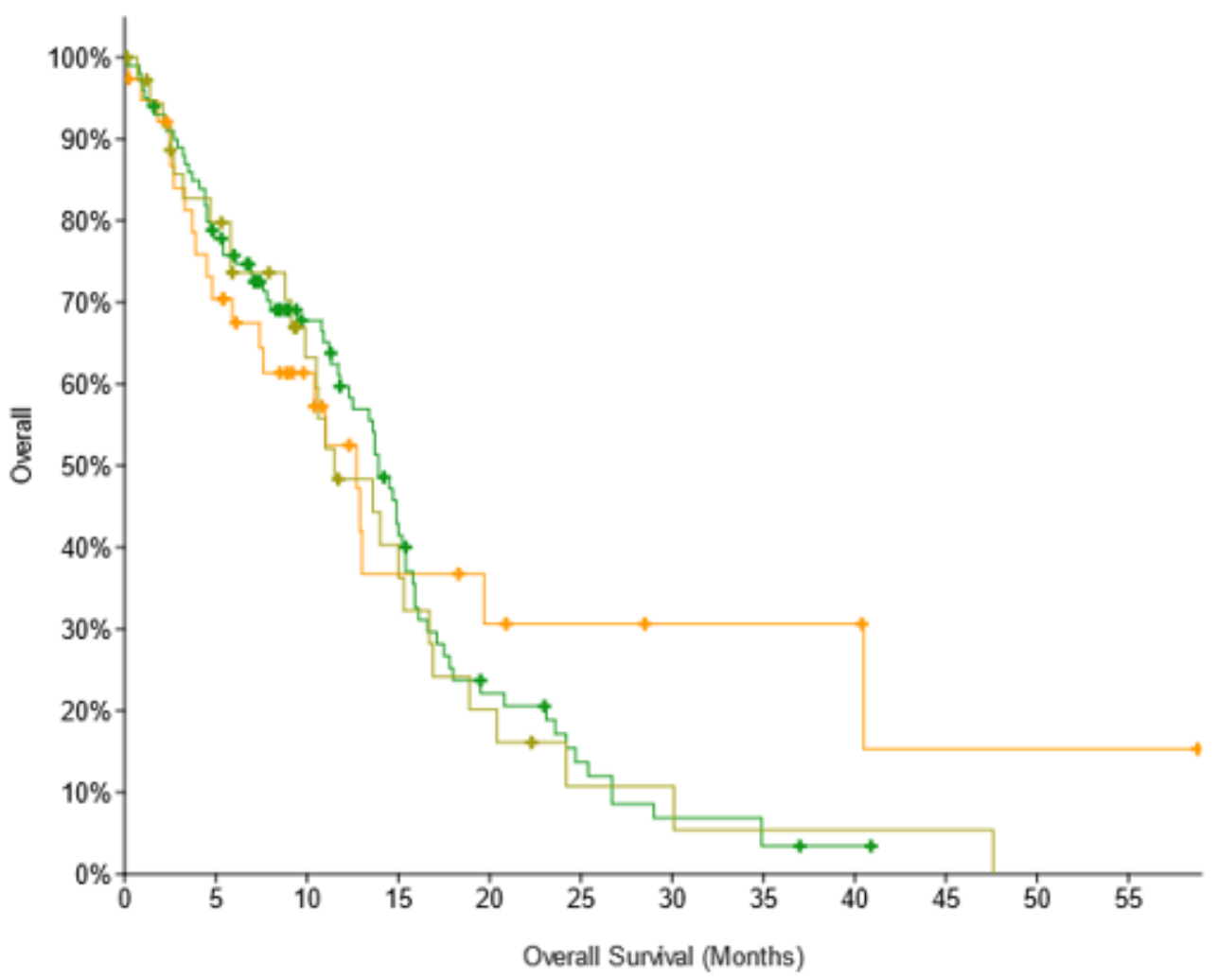

Overall

C Only PTEN

- Only EGFR

Only PTEN, EGFR

\begin{tabular}{ccc}
\hline & $\begin{array}{c}\text { Total } \\
\text { Cases }\end{array}$ & $\begin{array}{c}\text { Median Months } \\
\text { (Survival) }\end{array}$ \\
\hline Only PTEN & 38 & 12.70 \\
Only EGFR & 101 & 13.90 \\
PTEN \& EGFR & 37 & 11.50 \\
\hline
\end{tabular}

Figure 4.16: TCGA (2013) overall survival data for patients with EGFR and PTEN mutations. Kaplan Meier curves for overall survival as a percentage against months survived, for patients with mutations in PTEN and EGFR genes. Data from TCGA (2013) and figures from cBioPortal. 
While the data from the Pten + EGFR cell line is incomplete, the existing results corroborate the clinical picture. The density of the colonies in the clonogenic assay showed that the cells had a tendency towards proliferation. The C57C3 Pten cells showed a balance of proliferation and migration, and overall had a greater area of the plate covered. This mirrors what could be expected in the clinical setting; PTEN mutations alone progress faster, but because of the added proliferative advantage and increased cellular mass, patients with PTEN and EGFR co-mutations have an overall shortened survival. An interesting observation was the different trend in MTS assay that was seen in the Pten + EGFR cells. This cell line was the only one to differ in the trend of electron carrier production. The results from this assay implied that from days two to three, the Pten + EGFR cells increased the rate of production, whereas the C57C3 Pten and Pten + RAS results showed a steady gradient. While the overall results for these comparisons showed no significant differences, a future investigation of the metabolism of this cell line could shed some light on whether this could impact the tumorigenicity in vivo.

\subsection{Conclusion}

Overall, from the in vitro investigations conducted, all the 'classical-like' cell line models produced show promise in becoming a useful transplantation cell line. While the Pten + RAS cell line may from the typical GBM based on the genomic and molecular data, the results showed that the presence of the RAS V12 oncogene developed a more migratory cell line. This has the potential to better represent GBM in vivo than the currently used GL261 cell line. Comparatively, while the RAS + EGFR cell line was the least characterised, this combination of mutations is vitally important in modelling the classical subtype. The clonogenic assay results showed that this cell line had denser colonies than the C57C3 Pten cells. This indicated that the Pten + EGFR cells had a greater tendency towards proliferation than the C57C3 Pten cell line, which corroborates with what is seen in the pathology of the classical subtype, in human tumours. 


\subsection{Development and Characterisation of the 'Secondary-Like' GEM-CLeMs}

\subsection{Introduction}

Secondary GBM tumours arise much more slowly than de novo tumours, and do not tend to possess as many proliferation related driver mutations ${ }^{22,25}$. Additionally, secondary tumours that possess the IDH1 ${ }^{\mathrm{R} 132 \mathrm{H}}$ point mutation show an increased five-year survival rate ${ }^{130}$. For this objective, the human IDH1 ${ }^{\mathrm{R} 132 \mathrm{H}}$ was transfected into the C57F3 P53 cell line to develop a 'secondary-like' GEM-CLeM. These two cell lines were then compared with the astrocytes for glioblastoma-related characteristics, particularly survival, and dysregulation of the cell cycle.

\subsection{Addition of IDH1 ${ }^{\mathrm{R} 132 \mathrm{H}}$ to C57F3 P53}

\subsubsection{Stable transfection of the IDH1 $1^{R 132 H}$ oncogene}

The pcDNA3-Flag-IDH1-R132H plasmid was gifted by Yue Xiong ${ }^{100}$. The plasmid contained the coding sequences for the mutant IDH1 ${ }^{\mathrm{R} 132 \mathrm{H}}$ protein, and resistance cassettes for G418 and ampicillin (Fig 5.1). 


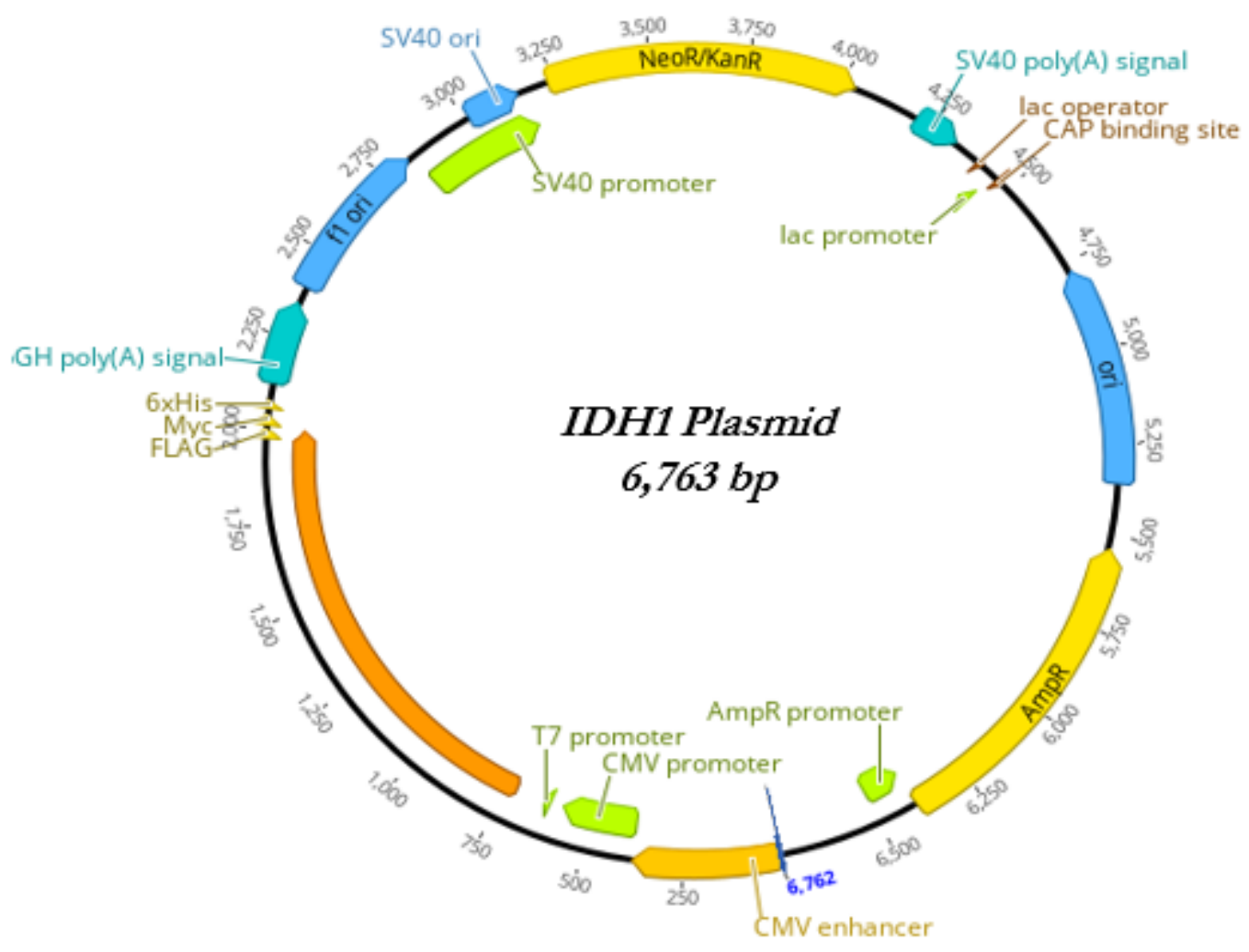

Figure 5.1: IDH1R132H mutant plasmid for stable transfection. Plasmid map for pcDNA3-Flag-IDH1-R132H. Plasmid contains IDH1 ${ }^{\mathrm{R} 132 \mathrm{H}}$ protein coding sequence (orange), ampicillin and G418 resistance cassettes (yellow) and promotor sequences (green). 
The C57F3 P53 line was transfected with the IDH1 ${ }^{\mathrm{R} 132 \mathrm{H}}$ plasmid. In parallel, a transfection was performed with tdTomato both as a positive control for the transfection and as a negative control for G418 selection. This transfection was successful as red fluorescence was emitted by the tdTomato positive cells. This was seen as yellow when visualised on the fluorescent microscope because of simultaneous tdTomato and EGFP emission (Fig 5.2). After treatment with G418, many cells died in both populations the following day. However, after replating the cells at day four, the tdTomato cells completely failed to adhere to the flask indicating they had not survived the treatment, while a portion of surviving IDH1 ${ }^{\mathrm{R} 132 \mathrm{H}}$ transfected cells could adhere. In figure 5.2, cells which had been transfected with tdTomato did not adhere after replating at day three. Whereas a proportion cells which were transfected with the IDH1 R132H plasmid did survive, seen with adherence at day five. This indicated that these cells had been successfully transfected (Fig 5.2). The IDH1 ${ }^{\mathrm{R} 132 \mathrm{H}}$ transfected cells were cultured to confluence in a T75 flask for two weeks, at which point they were harvested for RNA extraction.

Figure 5.2: G418 selection for IDH1R132H plasmid transfection. Compound microscopy pictures taken of C57F3 P53 cells after separate transfections with IDH1 and tdTomato plasmid. Brightfield images are shown for IDH1 transfection and overlaid FITC and TRITC fluorescent images taken for tdTomato Day 0. 


\section{IDH1 Transfection}
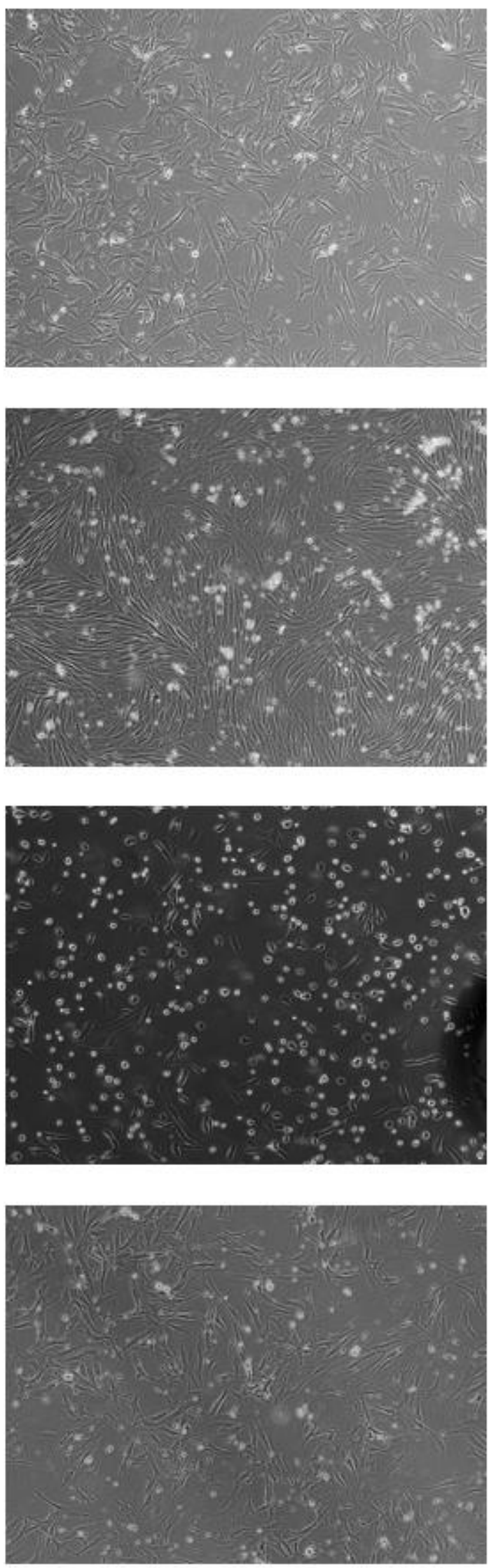

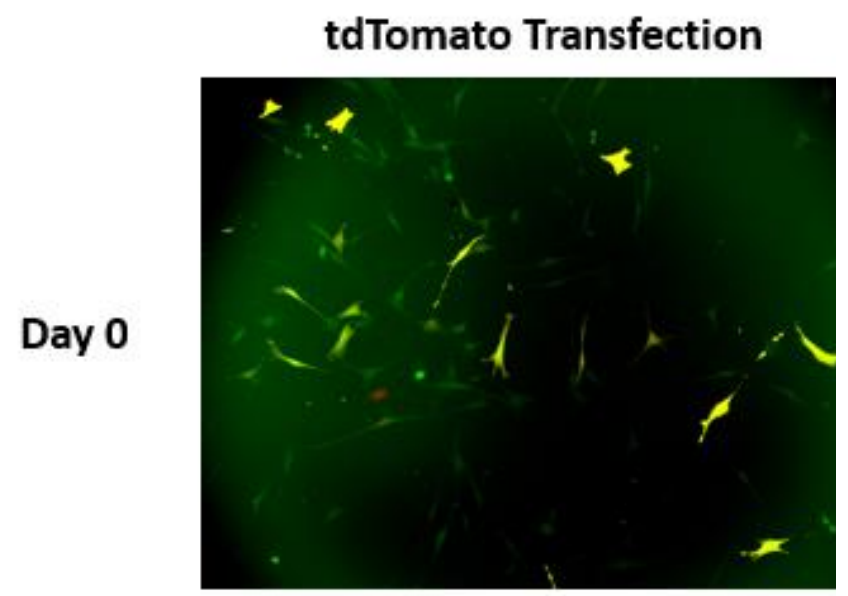

Day 3

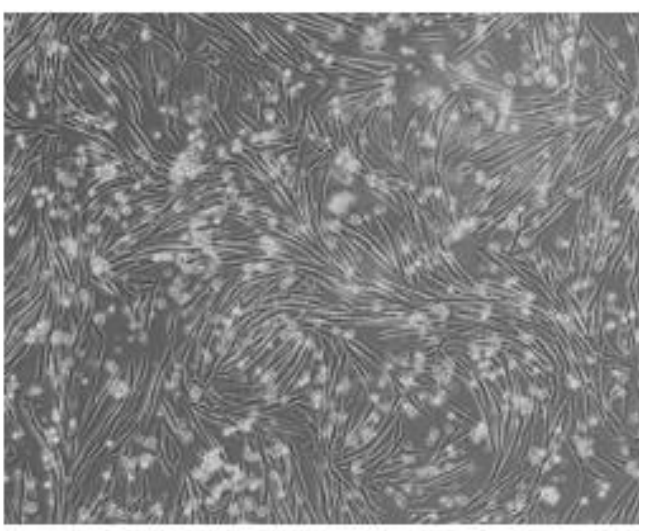

Day 5

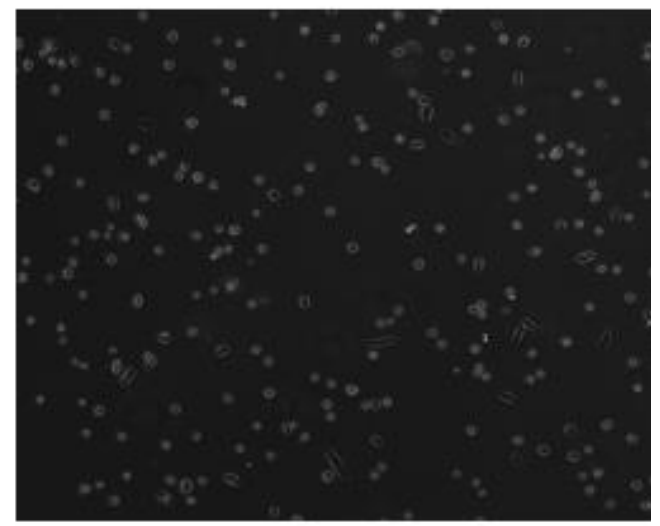

Day 10 


\subsubsection{RT-PCR to confirm IDH1 ${ }^{R 132 H}$ expression}

To confirm that the C57F3 P53 cells expressed the IDH1 ${ }^{\mathrm{R} 132 \mathrm{H}}$ oncogene, RT-PCR was conducted on the cDNA generated from the stably transfected cells. Primers targeting an approximately $200 \mathrm{bp}$ stretch at the start of the gene were created with no homology to the murine endogenous Idh1 gene. This RT-PCR confirmed that the C57F3 P53 cell line had been stably transfected with the IDH1 ${ }^{\mathrm{R} 132 \mathrm{H}}$ plasmid and that the cells were actively expressing the RNA (Fig 5.3). The constitutively expressed gene, HPRT, was used as a positive control for expression.

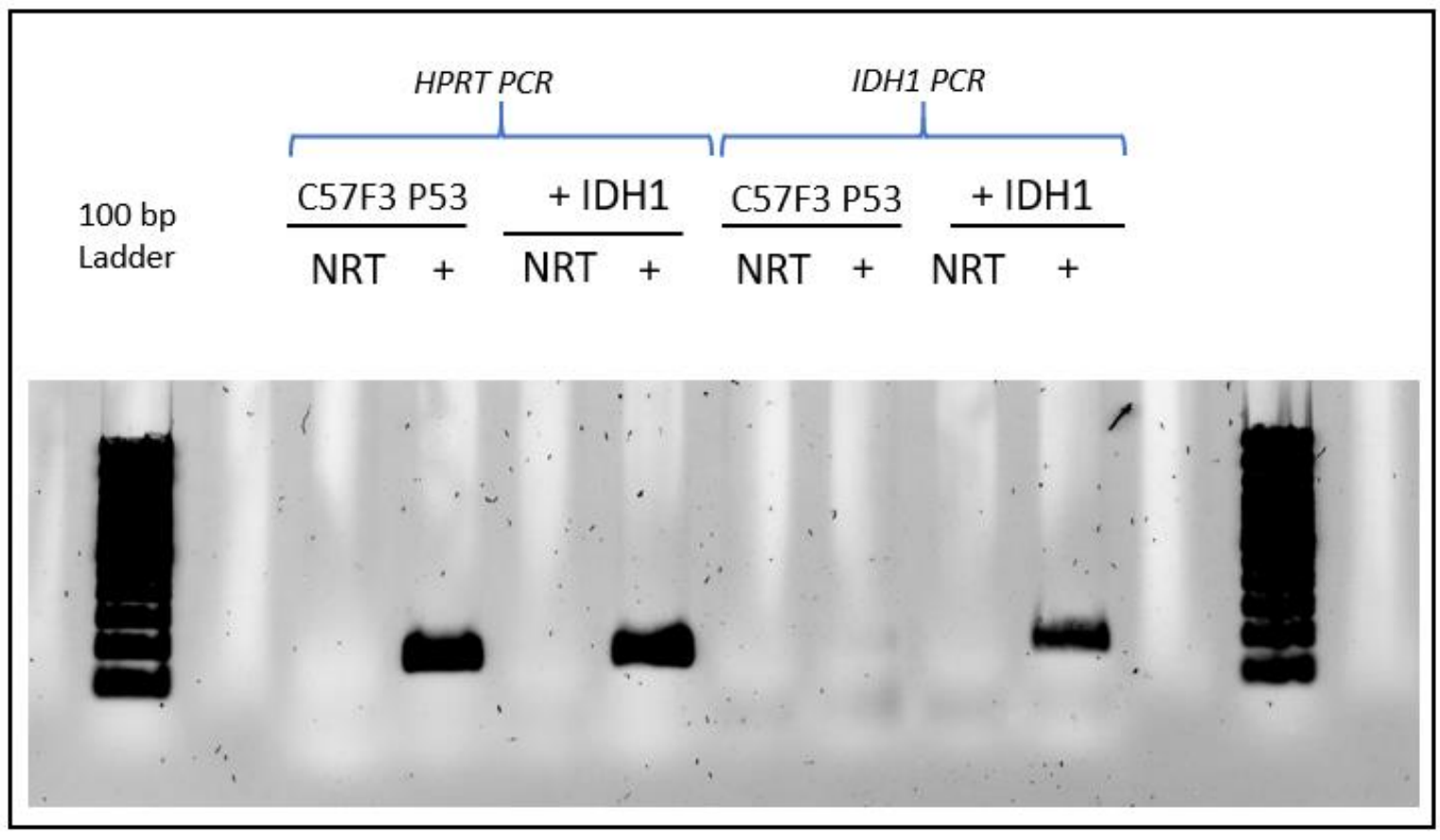

Figure 5.3: RT-PCR for IDH1R132H expression. 1.5\% TBE-agarose gel of RT-PCR products. cDNA from C57F3 P53 and IDH1R132H transfected cells were used. HPRT RT-PCR was a positive control for expression. 


\subsection{Functional Changes from P53 Mutation}

\subsubsection{P53 and dsDNA breaks}

One of the major roles of P53 is to arrest the cell cycle when dsDNA breaks occur in the genome. Initial DNA sensor proteins, such as ATM, register the damage and immediately phosphorylate P53, which is naturally inhibited by MDM2. This process stabilises P53, which increases its half-life and introduces a conformational change which allows the protein to act as a transcription factor. In particular, P53 is a transcription factor for senescence and apoptosis related proteins, such as P21 and Bax 106-108,131,132.

In a similar manner to chapter 3.3.7, which used radiation to induce P53 stabilisation, here radiation was used to induce cell cycle arrest. Using flow cytometry, the DNA content within a cell was used as a measure of which stage of the cell cycle the cells were in. This was achieved through fixing and permeabilising the cells, then staining with PI, a DNA-intercalating dye. The intensity of PI detected by flow cytometry was then used as a measure for DNA abundance. When the cells were in G1 phase (G1), the normal amount of DNA was detected. When the cells were in G2 and M phase (G2/M) there was double the amount due to the completion of the synthesis phase (S). This measurement was compared and quantified to investigate the functional changes the mutations had introduced. 


\subsubsection{Changes in cell cycle regulation in the C57F3 P53 cells}

After DNA damage was induced with 6 Gy of radiation, the stages of the cell cycle were compared between the astrocytes and the C57F3 P53 cells. The distribution of stages was uniform between the two cell lines when no radiation had been administered. Eight hours post-radiation, differences were observed. While both cell types had an increase in the proportion of G2/M cells (Fig 5.4A), the relative change in G2/M:G1 cells was different. The astrocytes showed approximately 4.1-times as many G2/M cells as G1, consistent with G2 arrest, the C57F3 P53 lines only showed 2.6-times as many cells in G2/M (Fig 5.4B). This result showed that C57F3 P53 cells had decreased induction of G2 arrest after ionising radiation.

Figure 5.4: Cell cycle assay after radiation for astrocytes and C57F3 P53 cells. Data from the cell cycle assay generated on FlowJo from astrocytes and C57F3 P53 cells. A. Histograms for PI intensity from flow cytometry for control and irradiated conditions. Cell cycle phases were colour coded: G1 phase (blue), S phase (brown) and G2/M phase (green). B. Percentage of cells in G2/M (orange) normalised to G1 (blue). Data for non-irradiated (-) and irradiated (+) cells. 
A

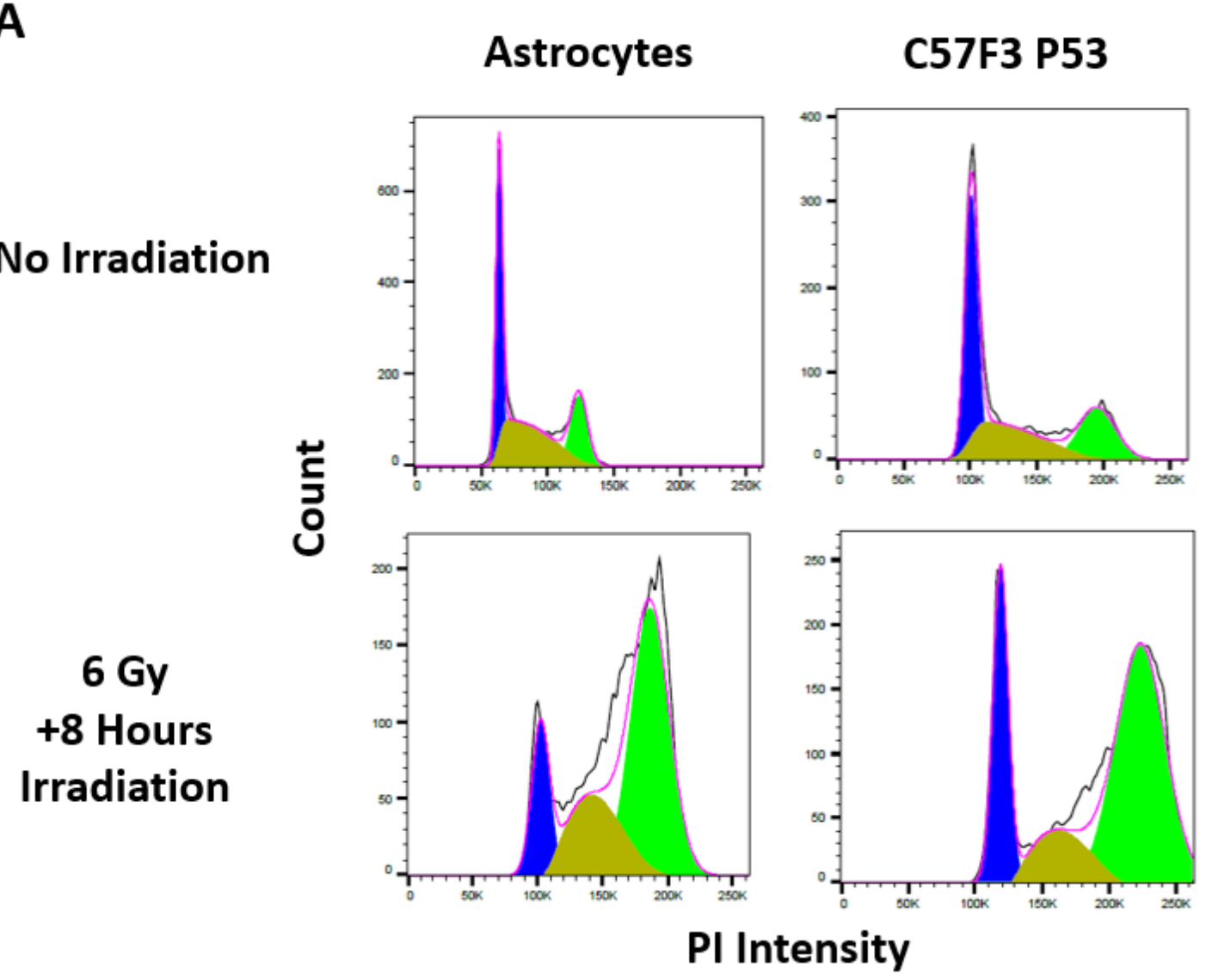

B

The effect of Radiation on G2/M Phase in the C57F3 P53 Cell Line

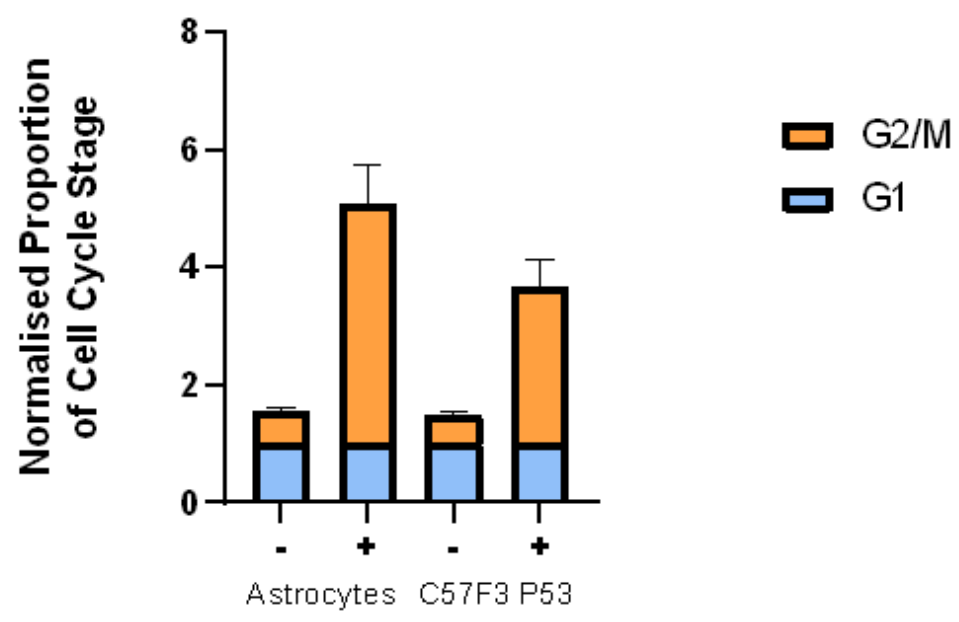

Cell Lines 


\subsubsection{Changes in cell cycle regulation in 'secondary-like' GEM-CLeMs}

The next objective was to compare the G2/M:G1 ratios in the C57F3 P53 lines with the P53 + IDH1 ${ }^{\mathrm{R} 132 \mathrm{H}}$ cells. As with the astrocytes, the P53 + IDH1 ${ }^{\mathrm{R} 132 \mathrm{H}}$ cells showed a very similar distribution of the cell cycle stages when no radiation had been given. Eight hours after radiation, the cells expressing the IDH1 mutation showed a visibly lower proportion of cells in G2/M compared with the C57F3 P53 line (Fig 5.5A). After normalising this data, the G2/M:G1 ratio showed that the P53 + IDH1 ${ }^{\mathrm{R} 132 \mathrm{H}}$ lines had only 1.6-times the number of cells in G2/M as G1 (Fig 5.5B), compared with the C57F3 P53 cells which showed 2.6-times. This result indicated that through the addition of IDH1R132H to the P53 knockout, the cells had further decreased their ability to arrest the cell cycle following DNA damage.

Figure 5.5: Cell cycle assay after radiation for 'secondary-like' GEM-CLeMs. Data from the cell cycle assay generated on FlowJo from C57F3 P53 and P53 + IDH1 cells. A. Histograms for PI intensity from flow cytometry for control and irradiated conditions. Cell cycle phases were colour coded: G1 phase (blue), S phase (brown) and G2/M phase (green). B. Percentage of cells in G2/M (orange) phase normalised to G1 (blue). Data for non-irradiated (-) and irradiated (+) cells. Error bars represent SEM, n=3 
A

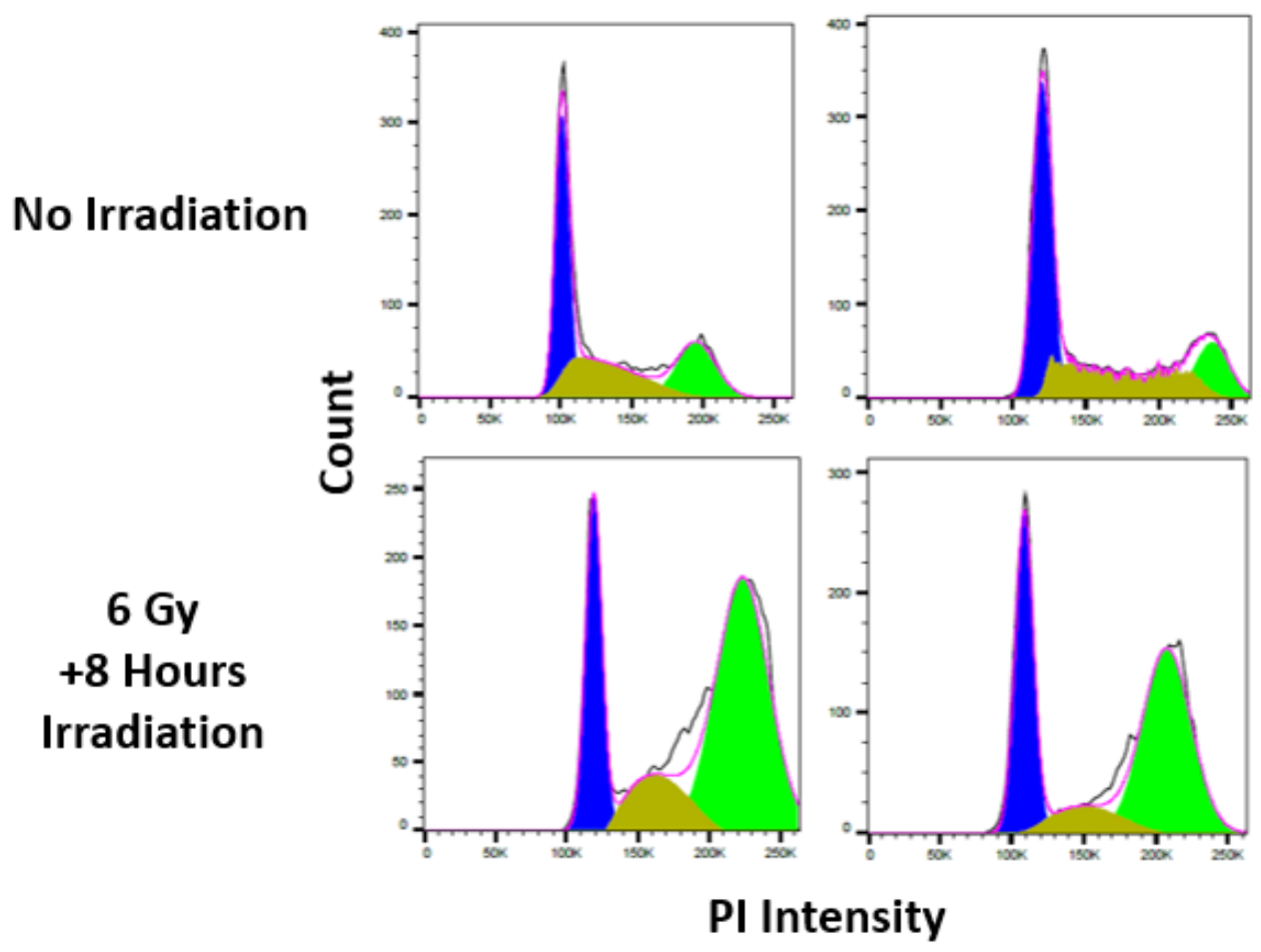

B

The effect of Radiation on G2/M Phase in the 'Secondary-like' Cell Lines

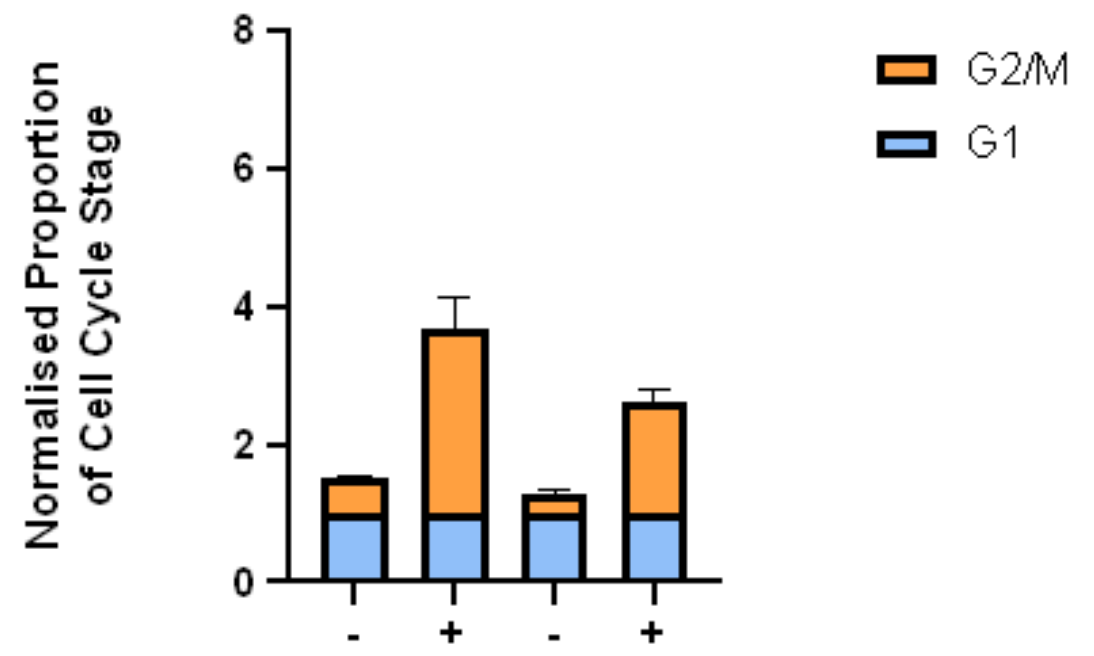

C57F3 P53 P53 + IDH1

Cell Lines 


\subsection{Characterising Proliferation Rates}

\subsubsection{Comparing C57F3 P53 and astrocyte proliferation rate}

A major observation made while culturing the C57F3 P53 cells was that these cells took longer to grow to confluence than the astrocytes. To quantify this, the proliferation rate was assessed using the Incucyte ${ }^{\circledR}$. Over four days, the C57F3 P53 cells grew to an average confluence of $45.21 \%(S E M=3.969, n=3)$ compared with the astrocytes which had an average confluence of $82.79 \%(S E M=0.728, n=3)$ (Fig 5.6A). Comparison of the AUC revealed that the astrocytes grew at a significantly faster proliferation rate, $t(6)=14.40$, $\mathrm{p}<0.0001(M=2909, S E M=95, n=3)$, compared with the C57F3 P53 cells $(M=1433$, SEM=39, $n=3$ ) (Fig 5.6B). 
A

Growth Curve Comparison

for Astrocytes Vs C57F3 P53

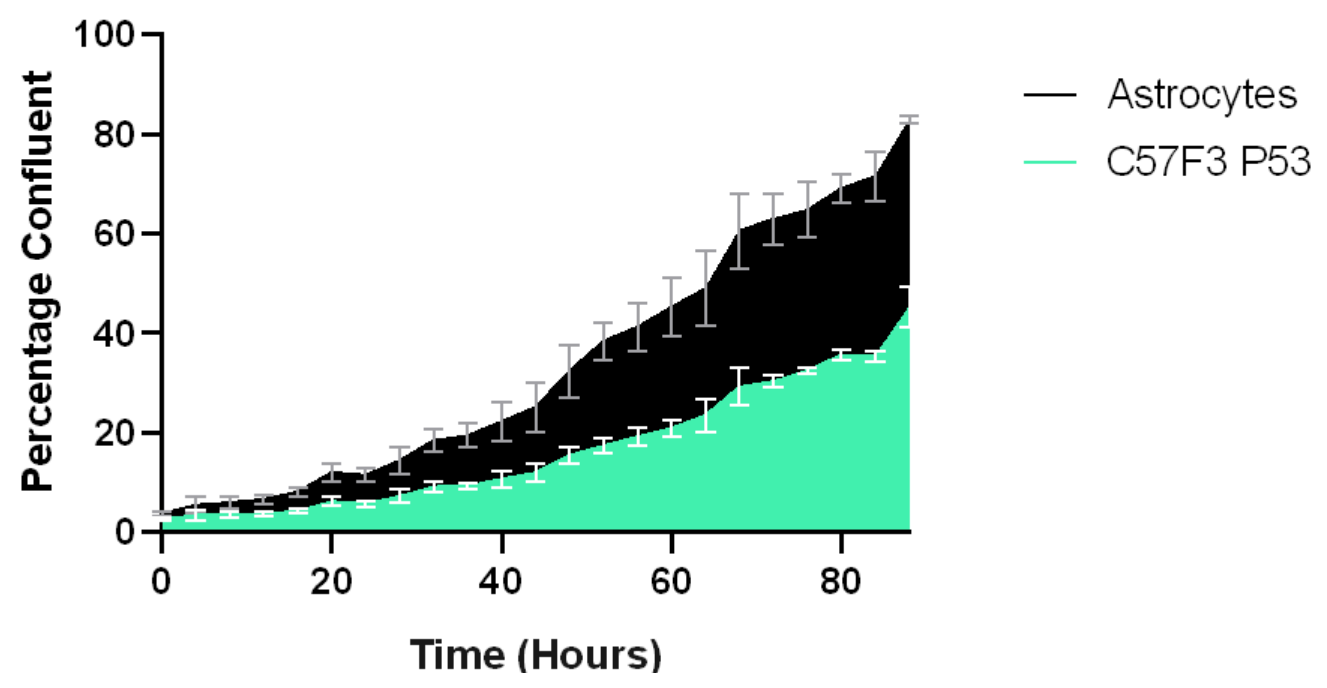

B

AUC of Growth Curve for Astrocytes Vs C57F3 P53

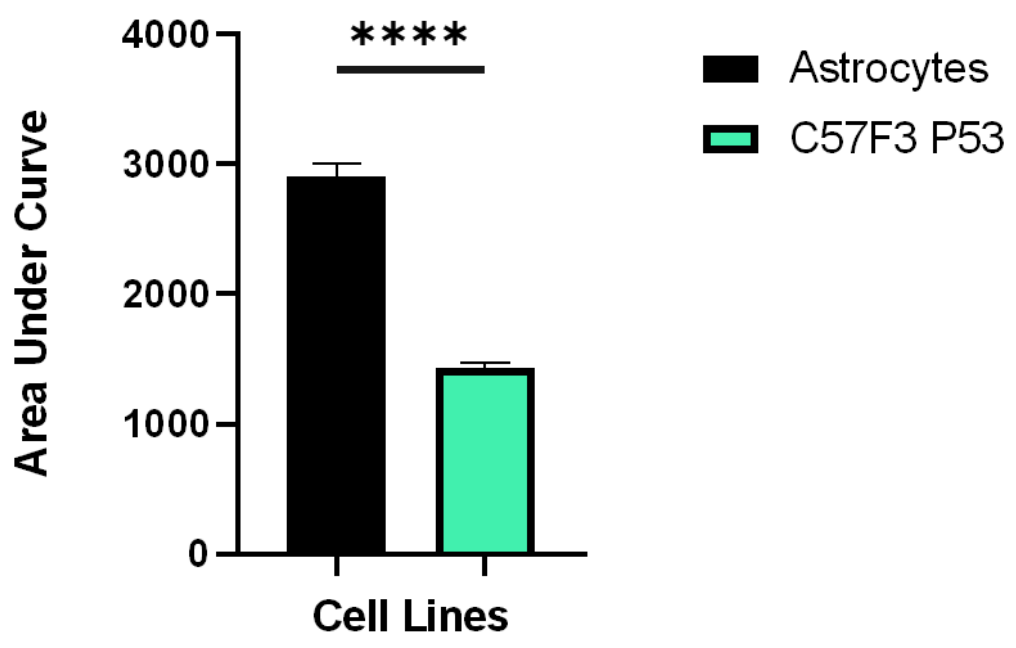

Figure 5.6: Proliferation assay for astrocytes and C57F3 P53 cells. Growth curve data for astrocytes (black) and C57F3 P53 cells (green), measured on the Incucyte ${ }^{\circledR}$. Data gathered over four-days. Error bars represent SEM, n =3. A. Confluence percentage against time (hours), measured every four hours. B. Area under curve (AUC) for confluence over time. ${ }^{* * * *}, \mathrm{p}<0.0001$. 


\subsection{Metabolic Assay}

\subsection{The 'secondary-like' GEM-CLeMs culture media pH level}

Unlike the C57C3 Pten line, there were no immediately obvious changes to the glycolytic activity of the C57F3 P53 and the P53 + IDH1 lines seen during culture of the cells. However, in order to assess whether any differences could be seen compared with the astrocytes, the $\mathrm{pH}$ of the DMEM at confluence was compared. At 100\% confluence, the C57F3 P53 media had a slightly higher $\mathrm{pH}(\mathrm{pH}$ 7.71) than the astrocytes ( $\mathrm{pH} 7.54)$, and consistent with the DMEM being slightly more pink. Comparatively, the P53 + IDH1 media had a higher $\mathrm{pH}(\mathrm{pH}$ 7.81) than the C57C3 P53 cells. Interestingly, at confluence the C57F3 P53 and P53 + IDH1 cells packed together less densely than the astrocytes (Fig 5.7). This data implied that there may be a slight difference in the methods of respiration between the lines; the 'secondary-like' lines may show a tendency towards oxidative phosphorylation, which would result in relatively less acid production. However, no conclusive differences were seen through the comparison of the media $\mathrm{pH}$.

Figure 5.7: $\mathrm{pH}$ Changes in the 'secondary-like' culture media $\mathrm{pH}$. Cell culture media, $\mathrm{pH}$, and brightfield microscopy image for astrocytes, C57F3 P53 and P53 + IDH1 cells, taken from cells at 100\% confluence. 


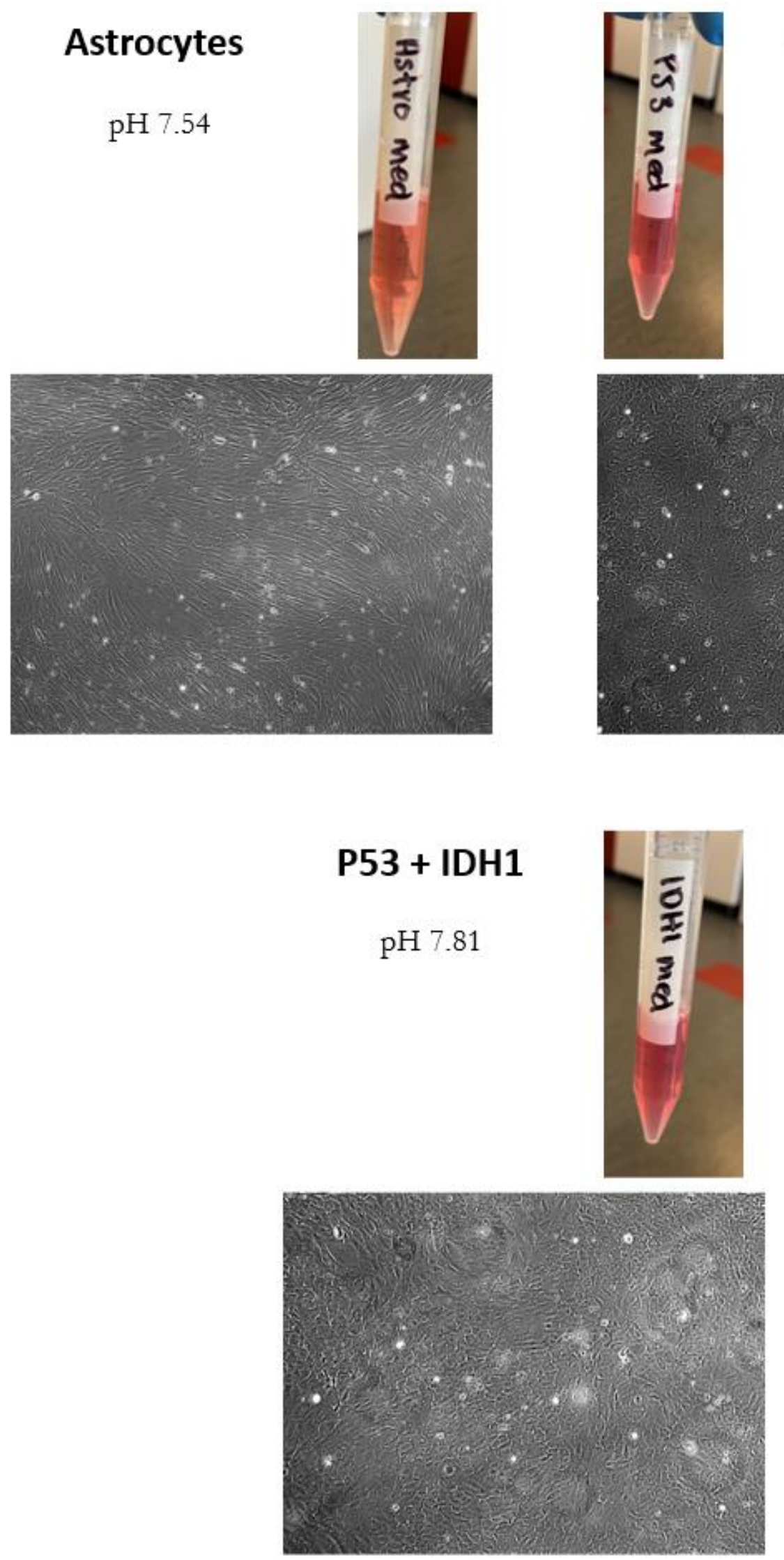

C57F3 P53

pH 7.71

P53 + IDH1

pH 7.81 


\subsubsection{Reductive activity of C57F3 P53 and astrocytes}

To investigate the nature of the $\mathrm{pH}$ differences observed, the MTS assay was conducted to compare the oxidative activity over time for the astrocytes and C57F3 P53 cells. Both showed the same trends seen in the C57C3 Pten line (4.6.2), with a steady rise in formazan concentration until day three and a slight decrease at day four. Differences were seen in the peak absorbance levels, with the astrocytes averaging 0.752 (SEM=0.028, $n=3$ ) and the C57F3 P53 averaging 0.548 (SEM=0.097, $n=3$ ); however, no statistical significance was seen in the differences in peak value, $t(16)=1.743, p=0.345$ (Fig 5.8A). To assess whether there was any significant difference in the rate at which the formazan was produced between the populations, the AUC were compared. This result also showed no significant difference between the two cell lines $t(16)=1.755, p=0.0985$ (Fig 5.8B). 


\section{A Absorbance from CellTitre MTS Assay for Astrocytes Vs C57F 3 P53}

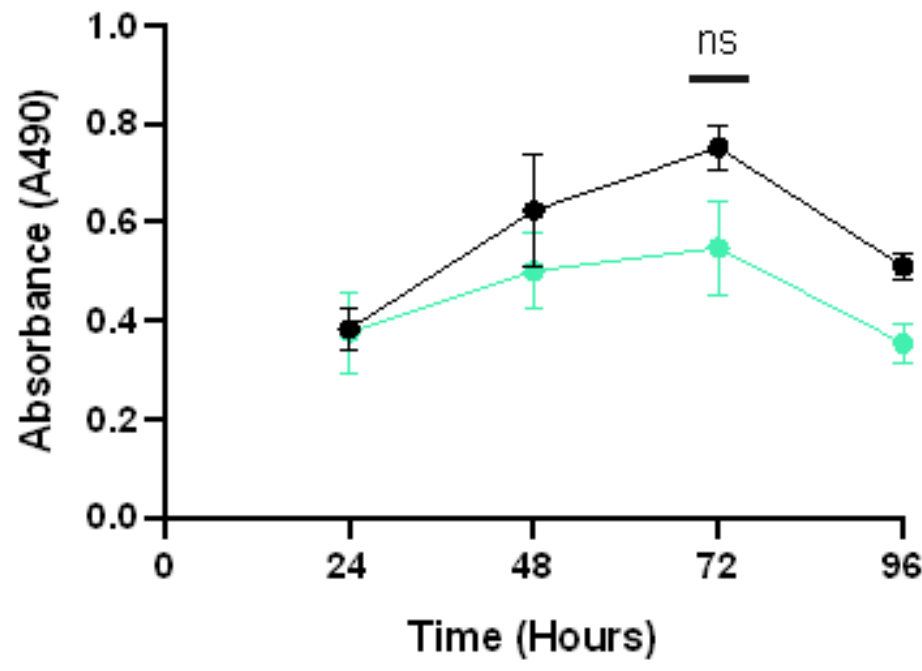

- Astrocytes

- C57F3 P53

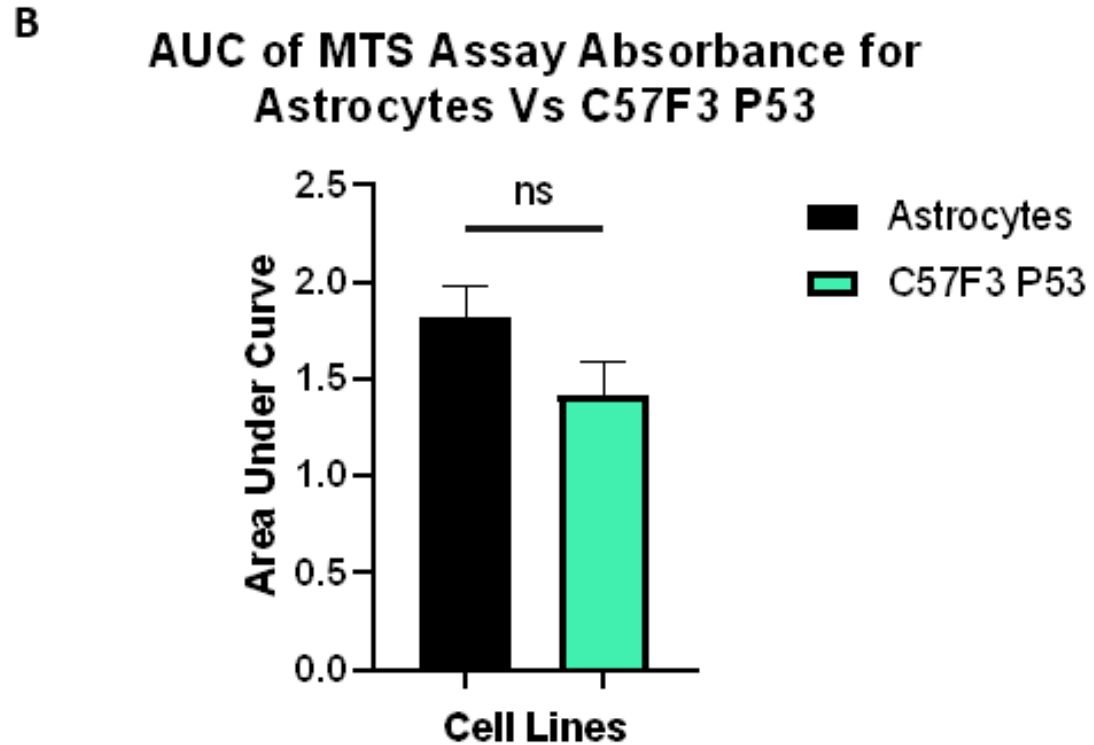

Figure 5.8: MTS Assay for astrocytes and C57F3 P53 cells. Error bars represent SEM and $n=3$. A. Absorbance of MTS assay measured over four days. ns, $p=0.345$. B. Area under curve (AUC) compared for astrocytes and C57F3 P53 cell lines. ns, $\mathrm{p}=$ 0.0985 . 


\subsubsection{Reductive activity of 'secondary-like' GEM-CLeMs}

In comparison, the P53 + IDH1 ${ }^{\mathrm{R} 132 \mathrm{H}}$ cell line showed a much flatter gradient in absorbance over the four-day assay, compared with the astrocytes and the C57F3 P53 cells. The absorbance peaked at day three $(M=0.449, S E M=0.070, n=3)$, however, this peak was not significantly different when compared with the C57F3 P53 line, $t(16)=0.277, p=0.697$. An interesting observation seen in this assay was that while the P53 + IDH1 R132H line showed much the same shape of graph as the C57F3 P53, and consistently averaged a lower absorbance, the final formazan concentration on day four was slightly higher $(M=0.383, S E M=0.037, n=3)$ compared with the cells from which they were derived $(M=0.354, S E M=0.041, n=3)$ (Fig 5.9A). To compare the rate of absorbance change, the AUC for the two lines were compared. This showed that there was no significant difference between the two cell lines, $t(16)=1.295, \mathrm{p}=0.214$ (Fig 5.9B). However, when comparing the AUC of the P53 + IDH1 ${ }^{\mathrm{R} 132 \mathrm{H}}$ line $(M=1.121, S E M=0.1471, n=3)$ with the astrocytes, a statistically significant difference was seen, $t(16)=3.262, p=0.0049$ (Fig 5.9C). This indicated that the rate of electron carrier production over time in the astrocytes did not significantly decrease with the P53 mutation but did so with the addition of IDH1R132H. 
A

Absorbance from CellTitre MTS Assay for C57F3 P53 Vs P53 + IDH1

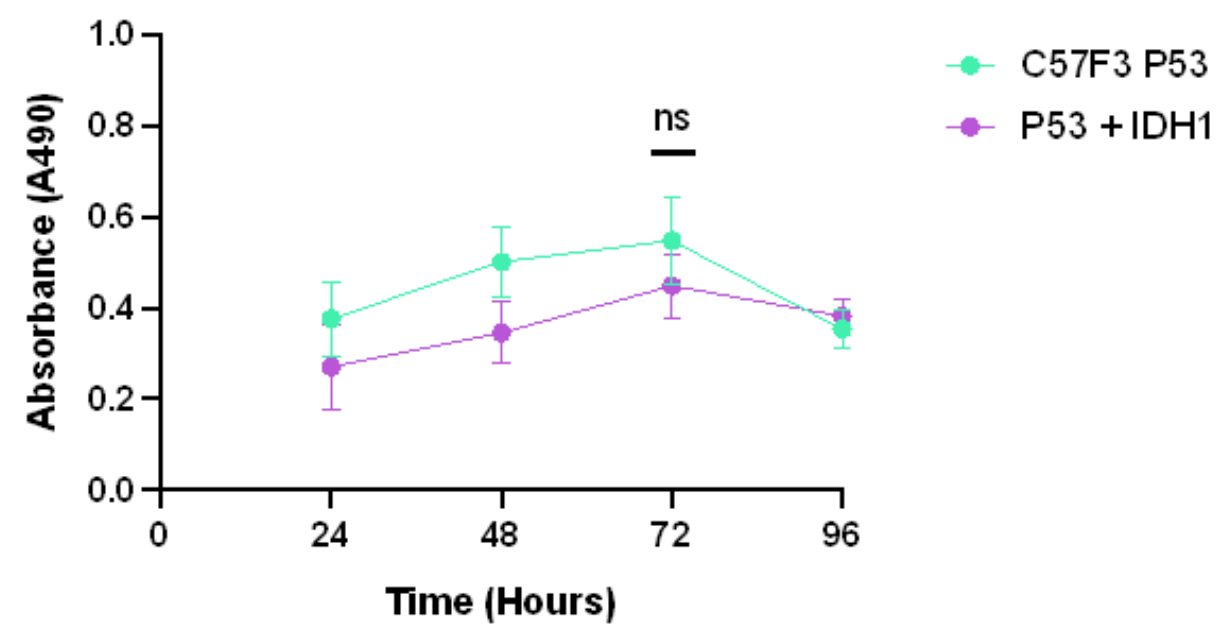

B

\section{AUC of MTS Assay Absorbance for C57F3 P53 Vs P53 + IDH1}

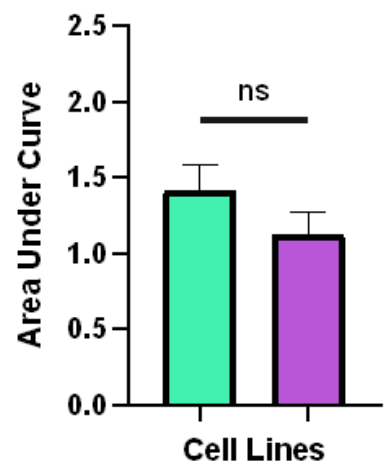

C

AUC of MTS Assay Absorbance for Astrocytes Vs P53 + IDH1

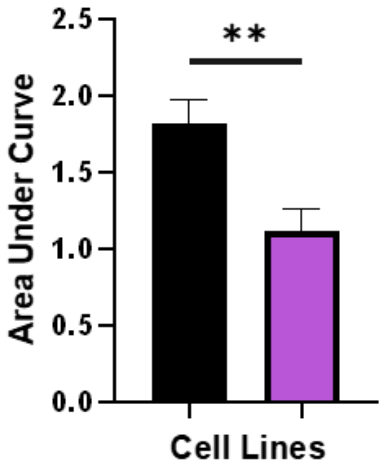

- Astrocytes

ㄷ53 + IDH1

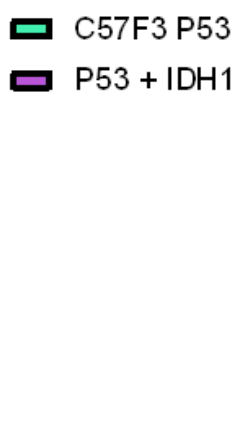

Figure 5.9: MTS Assay for 'secondary-like' GEM-CLeMs. Error bars represent SEM and $\mathrm{n}=3$. A. Absorbance of MTS assay for C57F3 P53 (green) and P53 + IDH1 12132H cells (pink), measured over four days. ns, $\mathrm{p}=0.697$. B. Area under curve (AUC) compared for C57F3 P53 and P53 + IDH1 cell lines. ns, $\mathrm{p}=0.214$. C. AUC compared for astrocytes (black) and P53 + IDH1 (pink) cell lines. **, $\mathrm{p}=0.00449$. 


\subsection{Clonogenic Potential of the Cell Lines}

\subsubsection{Comparing clonogenic abilities of the C57F3 P53 and astrocyte}

In order to assess whether the mutations made in the 'secondary-like' GEM-CLeMs have altered these cells' ability to form colonies, 15, 30 and 60 cells were plated in each well of a six-well plate and left to grow fourteen days. After two weeks of growth, it was seen that the C57F3 P53 cell line did not show any statistically significant differences in the ability to form a colony at all seeding densities, compared with the astrocytes; 15 cells, $t(2)=1.036, \mathrm{p}=0.4092 ; 30$ cells, $t(2)=1.413, \mathrm{p}=0.2932 ; 60$ cells, $t(2)=1.121, \mathrm{p}=0.3788$. All three seeding densities showed near identical relative area occupied (Fig 5.10B). However, it was observed that while the plating efficiency and relative area occupied were similar, overall, the C57F3 P53 colonies were denser than those of the astrocytes (Fig 5.10A). This observation implied that the P53 mutation had resulted in colonies which showed a greater amount of proliferation, relative to migration, than the astrocytes. 
A

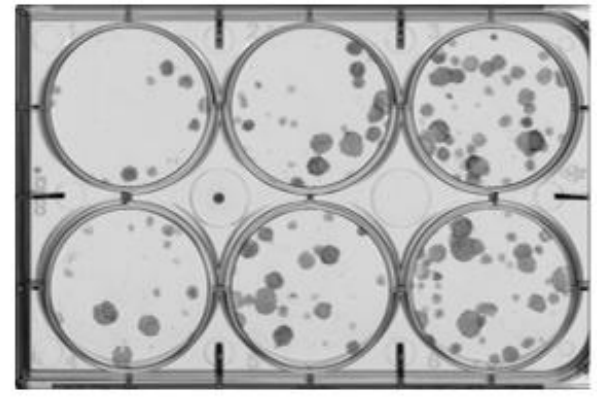

15 Cells 30 Cells 60 Cells
C57F3 P53

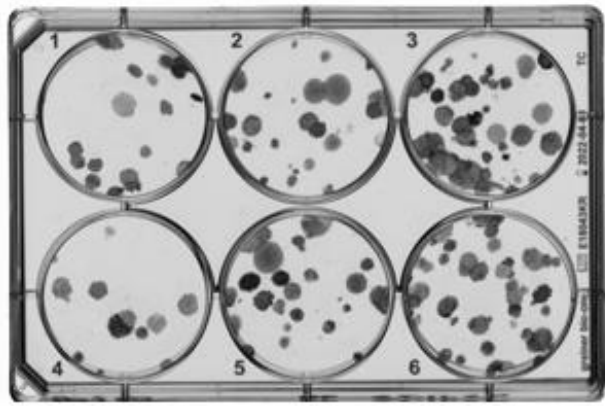

15 Cells 30 Cells 60 Cells

B

Clonogenic Assay for Astrocytes Vs C57F3 P53

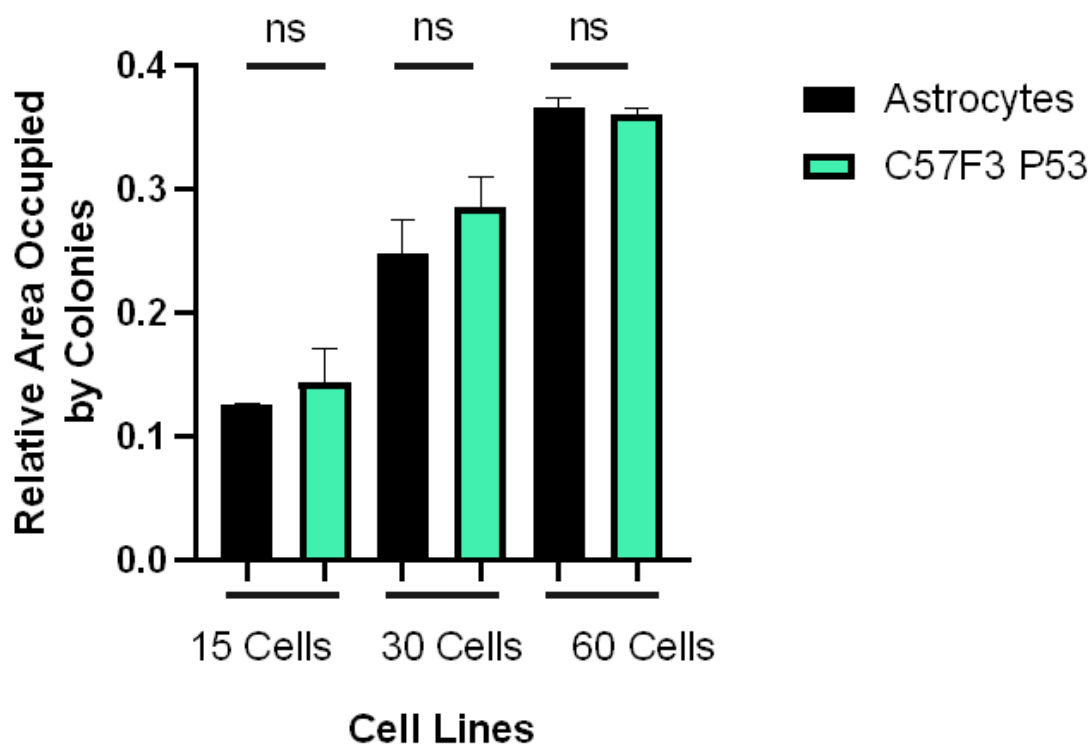

Figure 5.10: Clonogenic assay for astrocytes and C57F3 P53 cells. A. Methylene blue stained clonogenic assays after two weeks (representative of replicate data). Numbers refer to the cells seeded. B. Relative area of individual wells covered by colonies formed by the astrocytes (black) and C57F3 P53 cells (green), at each seeding density. Error bars represent SEM, n=3. Left to right, ns, p =0.4092, 0.2932, 0.3788. 


\subsubsection{Comparing the clonogenic abilities 'secondary-like' GEM-CLeMs}

When visually assessing the colony growth in the clonogenic assays, it was apparent that the P53 + IDH1 ${ }^{\mathrm{R} 132 \mathrm{H}}$ cells showed a lower level of clonogenic potential than the C57F3 P53 line when plated with 15 and 30 cells. Despite this, when statistically compared, the relative area occupied for the P53 + IDH1 ${ }^{\mathrm{R} 132 \mathrm{H}}$ cells was not significantly different to the C57F3 P53 line; 15 cells, $t(2)=1.522, p=0.2674 ; 30$ cells, $t(2)=2.683, p=0.1154 ; 60$ cells, $t(2)=0.9649, \mathrm{p}=0.4364$ (Fig 5.11B). The P53 + IDH1 ${ }^{\mathrm{R} 132 \mathrm{H}}$ colonies also appeared to be more spread-out and less dense compared with the C57F3 P53 cell line (5.11A). 


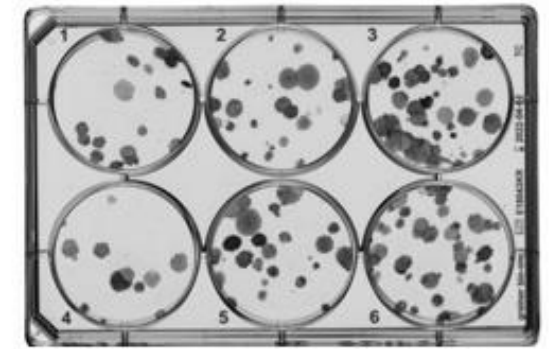

15 Cells 30 Cells 60 Cells

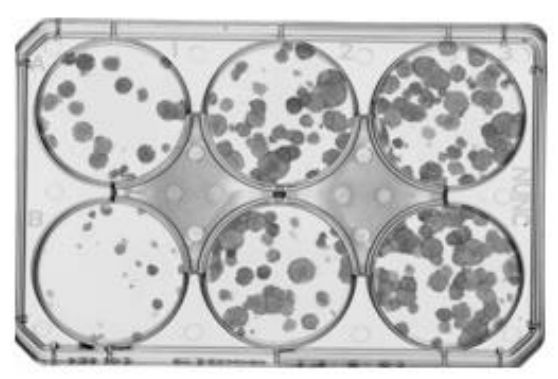

15 Cells 30 Cells 60 Cells

B

Clonogenic Assay for C57F3 P53 Vs P53 + IDH1

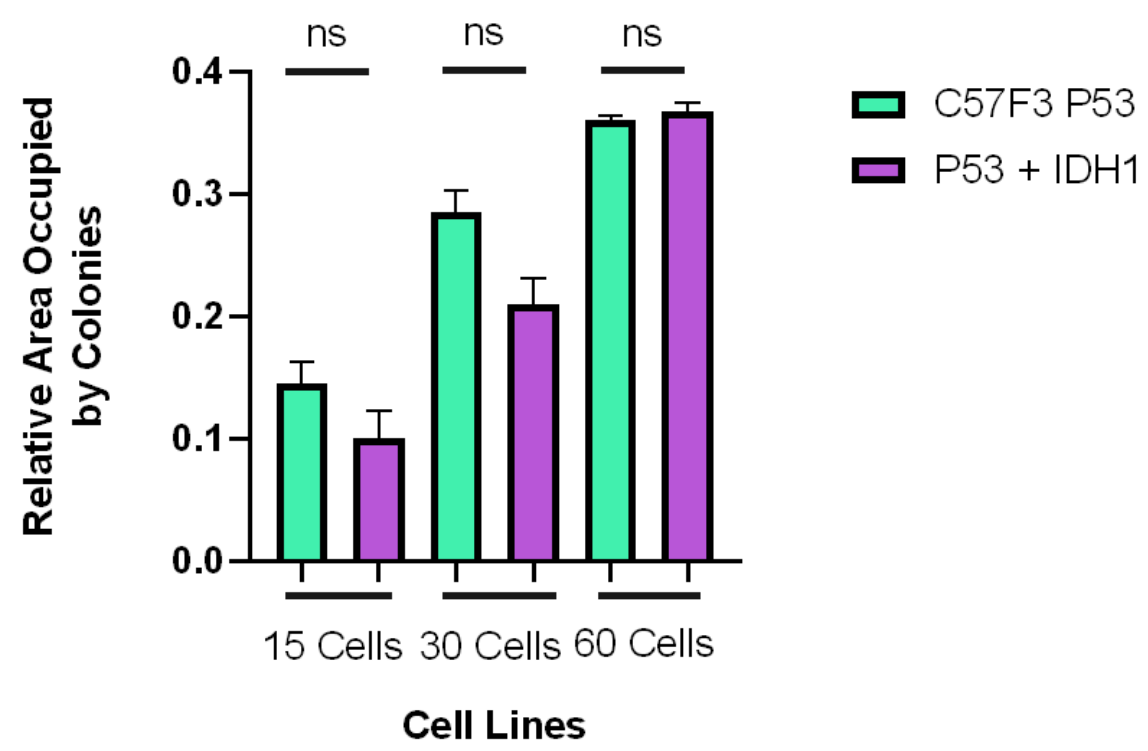

Figure 5.11: Clonogenic assay for 'secondary-like' GEM-CLeMs. A. Methylene blue stained clonogenic assays after two weeks (representative of replicate data). Numbers refer to the cells seeded. B. Relative area of individual wells covered by colonies produced by the C57F3 P53 cells (green) and P53 + IDH1 ${ }^{\mathrm{R} 132 \mathrm{H}}$ cells, at each seeding density. Error bars represent SEM, n=3. Left to right, ns, $\mathrm{p}=0.2674,0.1154,0.9649$. 


\subsection{Discussion}

This chapter had two objectives. Firstly, to develop a 'secondary-like' GEM-CLeM through the addition of the IDH1 ${ }^{\mathrm{R} 132 \mathrm{H}}$ oncogene to the C57F3 P53 line, and secondly, to characterise the effects that P53 knockout, and the additional IDH1132H transfection, had on the cell lines. Specifically, this characterisation was focused on the phenotypes of a secondary GBM. While the previous chapter compared the 'classical-like' cells' increased ability to proliferate and invade, this chapter investigated whether these proneural and secondary GBM specific mutations developed into a model with an increased cellular survival, gained through the loss of P53 function.

The C57F3 P53 cells immediately contrasted with the 'classical-like' GEM-CLeM as their proliferation rate was visibly lower than the astrocytes. This decrease was confirmed by comparing the rate of proliferation on the Incucyte ${ }^{\circledR}$. However, as with the previous chapter, this change did not appear to be indicative to the other results gathered. By normalising the results from the $\mathrm{C} 57 \mathrm{~F} 3 \mathrm{P} 53$ line to those from the astrocytes, an interesting trend was seen. The first observation was that while the proliferation rate of the P53 knockout line was significantly lower than the astrocytes, the metabolic activity was disproportionate to this change (Fig 5.12). The MTS assay had both cell lines plated at the same density to compare metabolic activity of the cell lines with as little variation as possible. Because of this, it was predicted that the trend in electron carrier production would follow that of the proliferation assay, as this was the factor known to differ between the cells. However, the MTS absorbance for these two cell lines was not significantly different when compared with the astrocytes. 


\section{Normalised Data for Astrocytes Vs C57F3 P53}

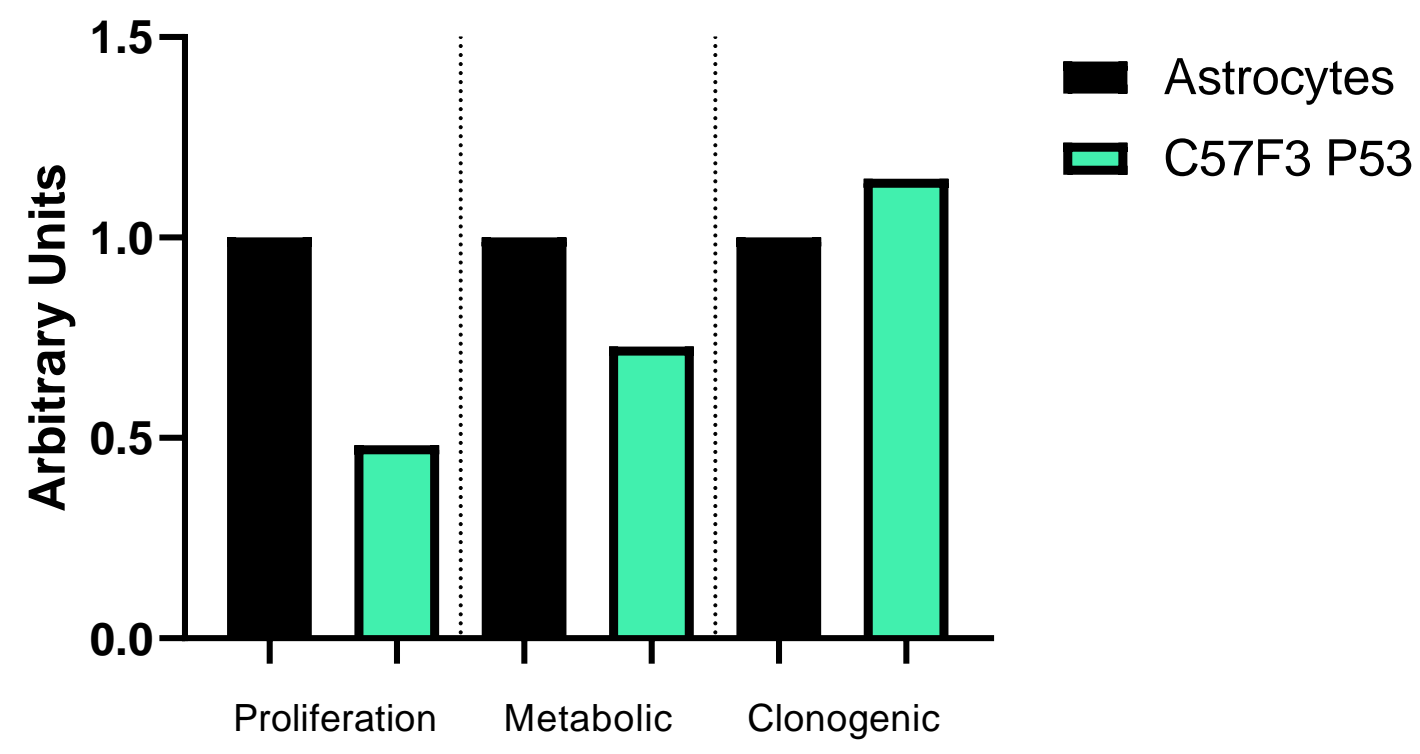

Cell Lines

Figure 5.12: Normalised data comparing the astrocyte and C57F3 P53 assays. Data gathered from the proliferation assay (5.4.1), MTS/metabolic assay (5.5.2), and clonogenic assays $\mathbf{5 . 6 . 1}, 15$ cells) shown when normalised to the results from the astrocytes.

This disparate relationship between proliferation and metabolism is likely to be due to the additional roles P53 plays in tumour suppression. Altered metabolism is a key hallmark of cancer ${ }^{133,134}$, and a major example of this is seen through the increased rate at which glucose is processed inside the tumour ${ }^{135}$. In a healthy cell, P53 is responsible for regulating glycolysis via inhibition of numerous GLUT channels and the initial enzyme of the catabolic pathway, hexokinase ${ }^{136}$. In the absence of P53, these proteins and many more are over expressed, leading to an increase in the rate of glycolysis 135,136. From the data generated in this chapter, I surmise that while the P53 knockout led to a decreased proliferation rate, the altered glycolysis regulation has increased the rate of electron carrier production.

The results from the clonogenic assay were the most impacted by the P53 indel. Unlike the proliferation and MTS assays, the clonogenicity of the C57F3 P53 appeared to 
be slightly greater than that of the astrocytes (Fig 5.12). While the results from this assay showed no statistical differences, this balance between proliferation and survival is crucial to understanding the cancer phenotype of the C57F3 P53 cell line. The metric used to analyse this assay was relative area occupied by the colonies. This measurement gave insight into both the seeding density, and the rate at which these cells grew under stress conditions. This result indicated that while the C57F3 P53 cells did not proliferate as rapidly under normal conditions, while experiencing stress, these cells grew as fast as the astrocytes. Visually, it appears that the number of colonies produced by the C57F3 P53 cells was similar to that of the astrocytes. If this experiment were to be replicated, the number of colonies produced could be measured alongside the area.

The differences between results in the growth curve and in the clonogenic assay are likely due to the absence of P53. It would be expected that this mutation has increased the C57F3 P53 cells survival in situations where the astrocytes would normally activate apoptotic or cell cycle arrest pathways. The most interesting observation was the increased density of the C57F3 P53 colonies. This result implied that compared with the astrocytes, the P53 knockout led to a tendency towards proliferation, rather than migration. This may be because of the absence of P53-related senescent signals. This result is promising in the context of tumorigenicity, as it implies that these cells are more likely to proliferate and grow in a stressed environment, such as the in vivo brain, than the astrocytes.

Unfortunately, due to the loss of the Incucyte ${ }^{\circledR}$, data for the P53 + IDH1 ${ }^{\mathrm{R} 132 \mathrm{H}}$ cell lines proliferation rate could not be gathered. However, through passaging of the cells, it was seen that this cell line had a slight decrease in the growth rate, not only compared with the astrocytes but also the C57F3 P53 line. This result was not unexpected. In heterozygous IDH1 point mutations, the protein's function overall is decreased. This is due to the enzymes requirement to dimerise. When the wildtype and $\mathrm{R} 132 \mathrm{H}$ proteins combine there is a dominant negative effect which decreases the production of $\alpha \mathrm{KG}$. Overall, tumours with IDH1 ${ }^{\mathrm{R} 132 \mathrm{H}}$ heterozygous mutations produce $4 \%$ the normal production of $\alpha \mathrm{KG}$. As a result, the tumours show a decrease in ATP production via the TCA cycle and oxidative phosphorylation, impacting the cells ability to proliferate. This situation was mimicked in the P53 + IDH1 ${ }^{\mathrm{R} 132 \mathrm{H}}$ cell line. The wildtype IDH1 gene was not knocked out, but instead 
IDH11 ${ }^{\mathrm{R} 132 \mathrm{H}}$ was overexpressed alongside. This would be expected to result in a decreased metabolic and proliferative rate, due to the dominant negative effect of the mutant protein. In the MTS and clonogenic assays, the P53 + IDH1 ${ }^{\mathrm{R} 132 \mathrm{H}}$ cells consistently showed slightly lower results than the C57F3 P53 line. Zhao et al. (2009), who developed the IDH1 plasmid, confirmed that the stable transfection of IDH1 ${ }^{\mathrm{R} 132 \mathrm{H}}$ alongside the wildtype protein induced decreased proliferation through a decrease in $\alpha \mathrm{KG}$ production 100. Therefore, I am confident that the results seen in these assays were due to the mutant enzyme.

The greatest difference that was seen in the analysis of the P53 + IDH1 ${ }^{\mathrm{R} 132 \mathrm{H}}$ cell line was through the cell cycle assay. The initial comparison between the astrocytes and the C57F3 P53 cells showed that after experiencing ionising radiation, the P53 knockout showed a decrease in the rate of G2 arrest. This was expected as the role of P53 in initiating cell cycle arrests after dsDNA breaks has been well reported 106,108,131,137,138. However, I did not anticipate that the addition of IDH1 ${ }^{\mathrm{R} 132 \mathrm{H}}$ to the P53 knockout would further increase the proportion of G1 cells. This may be due to the increase in histone and DNA methylation that the IDH1 ${ }^{\mathrm{R} 132 \mathrm{H}}$ oncogene induces. In IDH1 mutant GBM tumours, the massively increased DNA methylation and G-CIMP results in a significant decrease in transcription ${ }^{44,139}$. If cell cycle regulating transcription factors, such as P21, are expressed less frequently because of this methylator phenotype, it would be expected that the rate of cell cycle arrest would also decrease.

Moure et al. (2019) studied the effect that a deletion of a heterozygous IDH1 R132H would have on a human astrocytoma cell line. Using CRISPR/Cas, the exon containing the mutation was targeted and deleted, resulting in a loss in expression from both alleles. Following this, the genome-wide DNA methylation pattern was investigated to determine whether the removal of the R132H mutation affected the G-CIMP. Overall, specific CpG islands had a significant loss of DNA methylation because of the deletion ${ }^{139}$. This result showed that a heterozygous mutation in IDH1 is sufficient to induce a G-CIMP, not only in an induced cell line model as Zhao et al. (2009) demonstrated, but also in a patient derived glioma line with an endogenous IDH1 mutation. While an investigation into this was outside the scope of the project, future studies into the P53 + IDH1 ${ }^{\mathrm{R} 132 \mathrm{H}}$ cell line 
should focus on the G-CIMP. These could investigate whether there is widespread DNA methylation or whether there is a decrease in expression of additional cell cycle regulators.

Relative to the C57F3 P53 cell line, the colony growth for the P53 + IDH1 R132H line increased with the seeding density. While there were no significant differences seen between the relative area occupied by the two cell lines, when seeded with a greater number of cells, the two 'secondary-like' GEM-CLeMs showed greater similarity. This result is consistent with the MTS assay, because while the C57F3 P53 cells showed a decreased rate of electron carrier production, after four-days, and a high confluence had been reached, the absorbance was the same. This implied that as the cell number increased, so did the electron carrier production. Because of this, it was not unexpected that the clonogenicity also increased with an increased cell number.

The combination of P53 and IDH1 ${ }^{\mathrm{R} 132 \mathrm{H}}$ is an extremely clinically relevant model in GBM. P53 mutations are seen in 56 out of the 248 GBM tumour samples $(23 \%)$ in the latest TCGA study 27. This is a significant proportion and is important because P53 mutations are seen in a majority of proneural tumours. Comparatively, IDH1 mutations, of which $\mathrm{R} 132 \mathrm{H}$ is the most prevalent, account for only $6 \% 27$ (Fig 5.13). While this is a small proportion of tumours, it is reflective of approximately $90 \%$ of secondary GBM 130,140 . 
TP53

IDH1
$23 \%$

$6 \%$

1 1
Inframe Mutation (putative driver)

Splice Mutation (putative driver)
Missense Mutation (putative driver)

Truncating Mutation (putative driver)

Deep Deletion (putative driver)

\section{Figure 5.13: TCGA (2013) data on P53 and IDH1 mutations frequency in GBM.}

The bar represents all 248 tumour samples in the TCGA GBM database. Figure from cBioPortal.org

The presence of IDH1 mutants in GBM confers a slightly better prognosis than those with the wildtype protein. This is largely due to the decreased metabolic and proliferative capacity these cells possess, because of the impact on the ATP production. This relationship between IDH1 and improved prognosis can be seen in the overall survival data published by TCGA (2013). Tumours with P53 mutations and a wildtype IDH1 protein confer a similar median survival to that of PTEN (4.8), 12.5 months. However, when P53 and IDH1 are co-mutated, the median survival increases significantly (22.7 months) ${ }^{27}$ (Fig 5.14). While this is positive in terms of treatment, this combination of mutations remains tumorigenic and research needs to be conducted into efficient methods of therapy. Calvert et al. (2017) demonstrated that in the absence of proliferative driver mutations, P53/IDH1 tumours display an increased production of ROS and chromosome instability as a means of oncogenic progression ${ }^{39}$. It was proposed that the combination of ROS and the inhibition of demethylation in IDH1 mutant tumours drives the progression of these tumours. As a result, in the TCGA data, IDH1 is seen to be sufficient to induce GBM (Fig 5.14). 


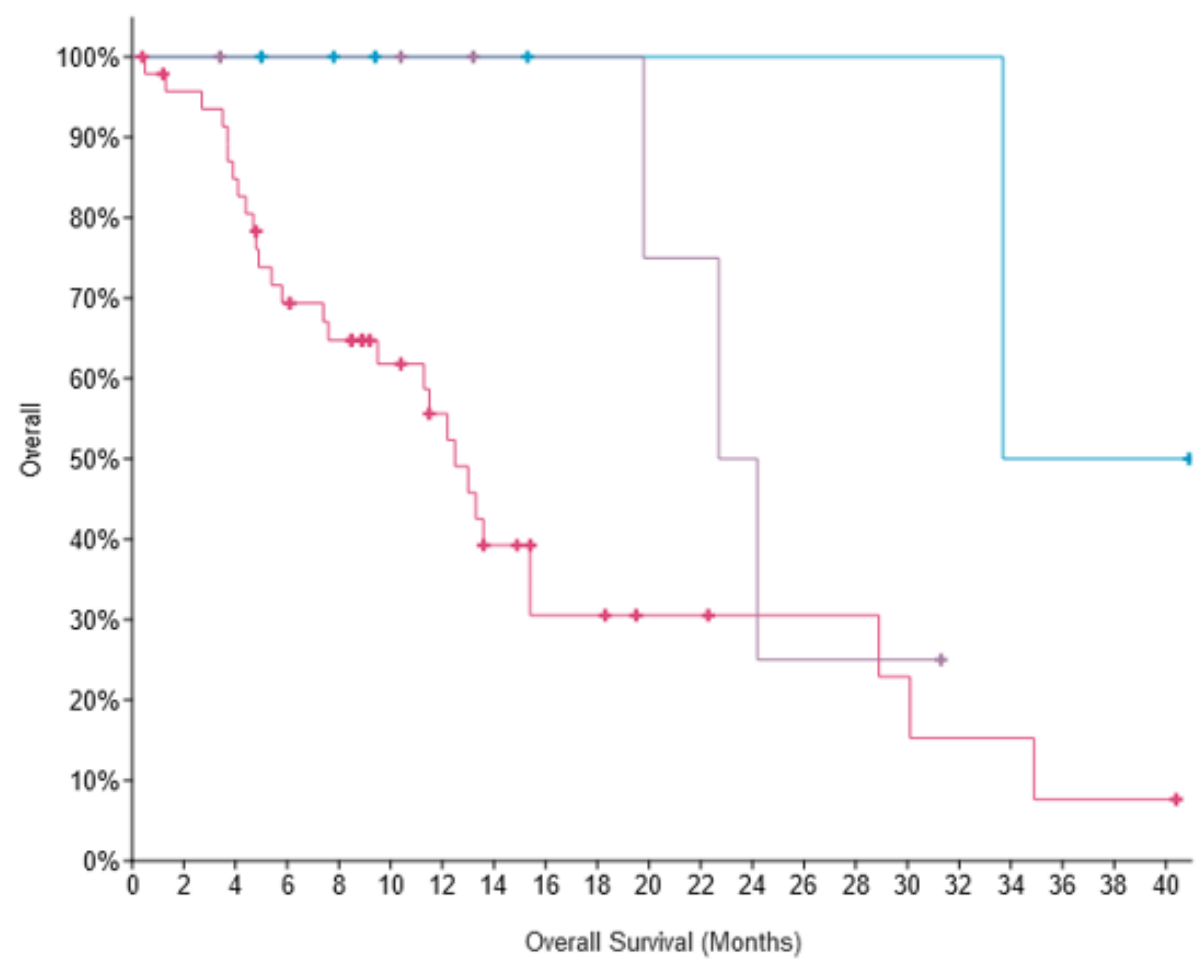

- Only IDH1

- Only TP53

- Only IDH1, TP53

\section{Total}

Cases

Median Months (Survival)

\begin{tabular}{ccc}
\hline Only P53 & 48 & 12.50 \\
\hline Only IDH1 & 6 & 33.70 \\
\hline P53 + IDH1 & 7 & 22.70 \\
\hline
\end{tabular}

Figure 5.14: TCGA (2013) overall survival data for patients with P53 and IDH1 mutations. Kaplan Meier curves for overall survival as a percentage against months survived, for patients with mutations in P53 and IDH1 genes. Data from TCGA (2013) and figures from cBioPortal. 


\subsection{Conclusion}

Currently, there are no readily available cell line models of secondary GBM other than PDX. Numerous studies have shown the slow growing tumorigenic effects that IDH1 mutations have in the brain, either through studying patient tissue or developing GEMMs 39,43,86,100,139-141. However, I believe that for more meaningful investigations into the biology and tumorigenicity of IDH1 mutant tumours, an immune competent, cost-effective model, which contains the clinically relevant mutation patterns, is needed. The results from C57F3 P53 and P53 + IDH1 ${ }^{\mathrm{R} 132 \mathrm{H}}$ confer with the survival data. Overall, the P53 knockout decreased the survival of the cells and disrupted the cell cycle regulation. With the addition of IDH1 ${ }^{\mathrm{R} 132 \mathrm{H}}$, the metabolic rate of the cells dropped, while further dysregulating the cell cycle. This indicated that while the P53 + IDH1 cell line is much slower growing than the C57F3 P53 cells, the relationship between the two appears to model what is seen in GBM tumours. Because of this, I believe that the 'secondary-like GEM-CLeMs will be an invaluable resource in investigations into IDH1 mutant GBM. 


\subsection{Final Discussion}

\subsection{Discussion}

As it stands, only $15 \%$ of brain tumour studies utilise GEMMs as a means of in vivo cancer modelling ${ }^{67}$. Despite the unique ability to develop tumours spontaneously in the brain of an immune competent mouse, this technique is not yet capable of representing the full complexity of GBM. Additionally, GEMMs remain too inaccessible due to their elevated cost and time-consuming development. Because of this, 97\% of brain tumour/GBM animal research involves the much more accessible transplantation models, either as the sole model or alongside GEMMs ${ }^{67}$. Because of this, the major limitation in translational GBM research is the inevitable crossroad of whether to transplant biologically relevant tissue (PDX), or murine cell lines into immune competent mice. Both models have their strengths, however, the weakness of not being able to harness both together results in a highly limited representation of GBM.

Future steps in in vivo gene editing may allow for appropriate and cost-effective models of GBM to be developed. But as is, the current GEMMs are too homogeneous and expensive to be utilised in a majority of research groups. However, the ability to genetically alter models has not been harnessed to its full extent. In vitro, genetic modification is a highly efficient and accurate technique. Because of the ability to target multiple genes within a single cell line, there is potential for in vitro gene editing to succeed where in vivo efforts have failed. This has led to a gap in GBM research which has the potential to address the limitations of the current models.

This study sought to create a novel model of glioblastoma which can be used to further the research in the field. The key aspects of this model were: a) it would be customisable - multiple mutations can be introduced, b) it could be transplanted into fully immune competent C57BL/6 mice - allowing for the full complexity of the tumour microenvironment to be used and c) it would be a cost effective model, with GBM specific 
mutations. An additional novel aspect these cells possess is the EGFP transgene. The expression of this fluorescent marker allows for the analysis of the tumour tissue to be separated from the 'healthy' parenchyma of the host mouse. Because of GBMs invasive nature, this aspect of the GEM-CLeMs is a distinct advantage over current transplantation models. Through analysing this fluorescence, GEM-CLeMs allow the opportunity for GBM animal models to study aspects of the tumour's progression, which are currently very difficult to achieve. For example, as no extra staining is required to visualise and distinguish the tumour from the host tissue, it will be easier to investigate parts of tumour progression, such as a) tumour invasion into healthy tissue and b) the tumours interaction with the surrounding stromal cells.

Five cell lines were created and characterised regarding how accurately they represented the GBM subtypes. Overall, the aims of this project were achieved. Two tumour suppressor knockout cell lines were developed using CRISPR/Cas9: C57C3 Pten and C57F3 P53. Using the Pten knockout line, two 'classical-like' GEM-CLeMs were created. The Pten + RAS cell line showed promising in vitro migration to model GBMs invasive nature, and the Pten + EGFR cell line lends itself as a much needed clinically relevant model of the classical subtype. With the stable transfection of an IDH1R132H oncogene plasmid, the P53 + IDH1 ${ }^{\mathrm{R} 132 \mathrm{H}}$ cell line was developed as a model for secondary GBM. These cells showed decreased levels of G2 arrest following ionising radiation, while also possessing a significantly decreased rate of electron carriers produced through glycolysis and oxidative phosphorylation. Currently, the plans for a pilot study are being developed, which will assess the in vivo tumorigenicity of the GEM-CLeMs. The models produced through this thesis will be intra-cranially implanted into immune competent C57BL/6 mice. Because of the results generated in vitro, I am confident in the tumorigenic potential these cell lines possess. The details of this study will be discussed in more depth later on (6.2.1).

The GEM-CLeM models created in this project show potential as a valid alternative to the current transplantation models. The current study aimed to model two distinct subtypes of GBM: the classical and secondary proneural. This investigation established and streamlined the necessary protocols for creating future GEM-CLeMs. For example, a 
mesenchymal model could be created through the knockout of Nf1 to the C57C3 Pten cell line. Additionally, a PDGFRA overexpression vector could be stably transfected into C57F3 P53 to model a primary proneural tumour.

The promising aspect of these models is their ability to be mixed together when used in vivo. Patel et al. (2014) showed the mosaic nature of GBM tumours through singlecell RNA-Seq ${ }^{60}$, and this concept has been particularly well documented in the proneural to mesenchymal transition following treatment 36,37,61,142,143. By transplanting multiple GEMCLeM 'subtypes' to provide the heterogeneity, this model has the potential to mimic GBM more accurately than GL261 and GEMMs. While PDX also possess this benefit, the combination of an intact immune response and the ability to curate the mutations to aid the research is a clear advantage of the GEM-CLeM.

These models do have limitations. The major benefit of GEMMs, which cannot be recapitulated through transplantation, is the spontaneous development of a tumour. This ability is extremely useful as it indicates how specific mutations influence the tumours development from a healthy cell to a cancer. This is a unique advantage which GEMMs possess and cannot be replicated through any means of transplantation. Similarly, PDX models have the benefit of containing the exact intracellular environment which results in the development of a GBM tumour. Through genomic and transcriptomic analysis, this can be modelled in vivo, however, molecular interactions inside the GEM-CLeMs are not guaranteed to copy that of the human tissue. For example, while Zhao et al. (2009) demonstrated that the IDH1 ${ }^{\mathrm{R} 132 \mathrm{H}}$ plasmid used in this project showed the expected decrease in $\alpha \mathrm{KG}{ }^{100}$, it is not known whether this mutant protein and D2HG are inducing tumorigenicity in the same manner as they do secondary tumour, as this has not been investigated.

While these examples show advantages of the current models over GEM-CLeMs, it is important to note that deciding the appropriate method of studying GBM is crucial. GEM-CLeMs would not be an appropriate choice in studies investigating spontaneous tumour growth or which require human tissue specifically. Instead, this model is appropriate for studies which wish to investigate the specific GBM tumour compositions in vivo, efficiently and in an immune competent model. GEM-CLeMs provide the 
opportunity for customisation and adaptation to a wide variety of research questions. Through the ability to add specific mutations to the model, these cell lines have an almost limitless potential in how they can aid GBM research.

\subsection{Future Investigations}

\subsubsection{In vivo and immunological studies}

In order to assess whether the mutations introduced to these cell lines have been sufficient to transform them and develop tumorigenicity, in vivo studies are necessary. Initially, this should be conducted as a pilot study involving the five cell lines created in this thesis. This would allow us to assess whether the mutations made were sufficient, or whether further alterations to the astrocytes are required. A crucial aim of this project was to develop a model which not only represents the pathophysiology of GBM, but also has the capacity to integrate with the immune system when transplanted in vivo. Because of this, future projects should be carried out in two parts: histological, and immunological analyses of the tumours. As a comparator, the in vivo work should include GL261 and PDX transplantations. It is important that the GEM-CLeMs are compared with PDX, as currently these are the only form of heterogeneous transplantation model. Similarly, as GL261 is the most frequently used murine cell line, it is important that the GEM-CLeMs ability to develop in an immune competent host is compared with the current option. As a result, this work would be directly comparable with the transplantation models for GBM which are currently in use.

Following the pilot study into the tumorigenicity of the current GEM-CLeMs, a 'mesenchymal-like' model should be developed. The co-deletion of PTEN and NF1 are the most frequently associated genes with the mesenchymal subtype, the presence of which is highly correlated with an increase in inflammation of the tumour. This was investigated by Wang et al. (2017), who identified microglia and macrophages using AIF1+ immunohistochemistry as a marker, after the transition from proneural to mesenchymal following the recurrence of GBM. The data showed that these immune cells were in closer 
proximity to NF1 negative cells, than to NF1 wildtype tissue. This associated the NF1 mutation with an enhanced immune infiltration, which is correlated with mesenchymal tumours ${ }^{61}$. Future GEM-CLeM studies could utilise the murine homolog, Aif1, to investigate the immune composition of the tumours developed. This could be coupled with a transcriptomic analysis of inflammation related transcription factors, such as Nf-kb and the Tnf family.

\subsubsection{Metabolic studies}

The ability of the P53 + IDH1 ${ }^{\mathrm{R} 132 \mathrm{H}}$ cell to produce D2HG is a crucial factor in modelling a secondary GBM. Unlike other driver mutations, IDH1 alterations decrease the growth and proliferation of the cells by altering the metabolism. However, in these tumours, the production of D2HG creates a unique intracellular environment which drives the tumour development. Because of this, an investigation into whether the P53+ IDH1 ${ }^{\mathrm{R} 132 \mathrm{H}}$ cell line produces an excess abundance of this oncometabolite should be conducted. Following this, the processes driven by the mutant IDH1 protein can be investigated in detail.

\subsubsection{Studies into BCL6 expression in GBM}

Previous experiments in the McConnell research group has demonstrated an inappropriate expression of the transcription factor, BCL6, in GBM. Primarily this protein

functions in lymphocytes and is essential in the process of $\mathrm{B}$ cell maturation, promoting anti-apoptotic and pro-survival pathways. However, Fabre et al. (2020) showed that following radiation, BCL6 is expressed in human GBM cell lines. This expression resulted in an increased survival of these cell lines in vitro ${ }^{144}$. BCL6 had previously been proposed as a therapeutic target in GBM. Xu et al. (2017) demonstrated that the RTK, AXL, was a transcriptional target of BCL6. In transgenic mouse models, it was shown that silencing of BCL6 and AXL, independently, increased the overall survival ${ }^{145}$. Unpublished data from our group has found, through ChIP-Seq, that in the human GBM cell line, LN18, BCL6 
was seen to target the EGFR locus. This indicated the potential for BCL6 to induce expression of the proliferation generating receptor.

This area of BCL6 in GBM demonstrates potential for using GEM-CLeMs to understand novel drivers and create targeted treatments. Future work with the Pten + EGFR cell line should investigate the effect of BCL6 on this mutational background. This could be conducted by stably transfecting a BCL6-overexpression vector into the Pten + EGFR line, and using ChIP-Seq to assess whether this interaction between the EGFR loci and BCL6 is seen in vitro. Following this, studies into whether BCL6 is a valid therapeutic target of GBM could utilise this model.

\subsection{Conclusion}

The development of these GEM-CLeMs introduces the potential for a valid alternative to the current GBM animal models. The in vitro assays conducted in this thesis show that the 'classical-like' and 'secondary-like' models recapitulate many of the characteristics seen in cancer cells and will be very useful in investigating a wide array of topics in GBM research. The next steps with the GEM-CLeMs will be to begin studies with intra-cranial transplantation, to investigate whether the positive results from this project correlate with in vivo tumorigenicity. 


\section{References}

1. Jessen, K. R. Glial cells. Int. J. Biochem. Cell Biol. 36, 1861-1867 (2004).

2. Allen, N. J. \& Barres, B. A. Glia - more than just brain glue. Nature 457, 675-677 (2009).

3. Molofsky, A. V. \& Deneen, B. Astrocyte development: A guide for the perplexed. Glia 63, 1320-1329 (2015).

4. Mahmoud, S., Gharagozloo, M., Simard, C. \& Gris, D. Astrocytes maintain glutamate homeostasis in the CNS by controlling the balance between glutamate uptake and release. Cells 8, (2019).

5. Kuhn, S., Gritti, L., Crooks, D. \& Dombrowski, Y. Oligodendrocytes in development, myelin generation and beyond. Cells 8, (2019).

6. Jessen, K. R., Mirsky, R. \& Lloyd, A. C. Schwann cells: Development and role in nerve repair. Cold Spring Harb. Perspect. Biol. 7, a020487 (2015).

7. Nayak, D., Roth, T. L. \& McGavern, D. B. Microglia development and function. Annu. Rev. Immunol. 32, 367-402 (2014).

8. Hines, P. J. Specialization in brain neurogenic niche. Science 356, 1346-1348 (2017). 
9. Bready, D. \& Placantonakis, D. G. Molecular pathogenesis of low-grade glioma. Neurosurg. Clin. N. Am. 30, 17-25 (2019).

10. Chen, R., Smith-Cohn, M., Cohen, A. L. \& Colman, H. Glioma subclassifications and their clinical significance. Neurotherapeutics 14, 284-297 (2017).

11. Nørøxe, D. S., Poulsen, H. S. \& Lassen, U. Hallmarks of glioblastoma: a systematic review. ESMO Open 1, (2017).

12. Hervey-Jumper, S. L. \& Berger, M. S. Maximizing safe resection of low- and highgrade glioma. J. Neurooncol. 130, 269-282 (2016).

13. Aldape, K., Zadeh, G., Mansouri, S., Reifenberger, G. \& von Deimling, A. Glioblastoma: pathology, molecular mechanisms and markers. Acta Neuropathol. (Berl.) 129, 829-848 (2015).

14. Ahir, B. K., Engelhard, H. H. \& Lakka, S. S. Tumor development and angiogenesis in adult brain tumor: Glioblastoma. Mol. Neurobiol. 57, 2461-2478 (2020).

15. Phillips, H. S. et al. Molecular subclasses of high-grade glioma predict prognosis, delineate a pattern of disease progression, and resemble stages in neurogenesis. Cancer Cell 9, 157-173 (2006).

16. Rong, Y., Durden, D. L., Van Meir, E. G. \& Brat, D. J. 'Pseudopalisading' necrosis in glioblastoma: A familiar morphologic feature that links vascular pathology, hypoxia, and angiogenesis. J. Neuropathol. Exp. Neurol. 65, 529-539 (2006). 
17. Capdevila, C., Vázquez, L. R. \& Martí, J. Glioblastoma multiforme and adult neurogenesis in the ventricular-subventricular zone: A Review. J. Cell. Physiol. 232, 1596-1601 (2017).

18. Stupp, R. et al. Radiotherapy plus concomitant and adjuvant temozolomide for glioblastoma. N. Engl. J. Med. 352, 987-996 (2005).

19. Delgado-López, P. D. \& Corrales-García, E. M. Survival in glioblastoma: a review on the impact of treatment modalities. Clin. Transl. Oncol. Off. Publ. Fed. Span. Oncol. Soc. Natl. Cancer Inst. Mex. 18, 1062-1071 (2016).

20. Jiapaer, S., Furuta, T., Tanaka, S., Kitabayashi, T. \& Nakada, M. Potential strategies overcoming the temozolomide resistance for glioblastoma. Neurol. Med. Chir. (Tokyo) 58, 405-421 (2018).

21. al, G. D., et. Divergent evolution of temozolomide resistance in glioblastoma stem cells is reflected in extracellular vesicles and coupled with radiosensitization. - PubMed - NCBI. https://www.ncbi.nlm.nih.gov/pubmed/29016925.

22. Verhaak, R. G. W. et al. Integrated genomic analysis identifies clinically relevant subtypes of glioblastoma characterized by abnormalities in PDGFRA, IDH1, EGFR, and NF1. Cancer Cell 17, 98-110 (2010).

23. Yuan, F. et al. Identification of key pathways and genes in the Orai2 mediated classical and mesenchymal subtype of glioblastoma by bioinformatic analyses. Dis. Markers 2019, 7049294 (2019). 
24. Campos, B., Olsen, L. R., Urup, T. \& Poulsen, H. S. A comprehensive profile of recurrent glioblastoma. Oncogene 35, 5819-5825 (2016).

25. Ohgaki, H. \& Kleihues, P. The definition of primary and secondary glioblastoma. Clin. Cancer Res. Off. J. Am. Assoc. Cancer Res. 19, 764-772 (2013).

26. McLendon, R. et al. Comprehensive genomic characterization defines human glioblastoma genes and core pathways. Nature 455, 1061-1068 (2008).

27. Brennan, C. W. et al. The somatic genomic landscape of glioblastoma. Cell 155, 462477 (2013).

28. Li, J. et al. PTEN, a putative protein tyrosine phosphatase gene mutated in human brain, breast, and prostate cancer. Science 275, 1943-1947 (1997).

29. Zheng, H. et al. p53 and Pten control neural and glioma stem/progenitor cell renewal and differentiation. Nature 455, 1129-1133 (2008).

30. Hopkins, B. D., Hodakoski, C., Barrows, D., Mense, S. \& Parsons, R. E. PTEN function, the long and the short of it. Trends Biochem. Sci. 39, 183-190 (2014).

31. Georgescu, M.-M. PTEN tumor suppressor network in PI3K-Akt pathway control. Genes Cancer 1, 1170-1177 (2010).

32. An, Z., Aksoy, O., Zheng, T., Fan, Q.-W. \& Weiss, W. A. Epidermal growth factor receptor and EGFRvIII in glioblastoma: signaling pathways and targeted therapies. Oncogene 37, 1561-1575 (2018). 
33. Liu, X., Chu, Y., Wang, D., Weng, Y. \& Jia, Z. MAPK-mediated upregulation of fibrinogen-like protein 2 promotes proliferation, migration, and invasion of colorectal cancer cells. Cell Biol. Int. 43, 1483-1491 (2019).

34. Asati, V., Mahapatra, D. K. \& Bharti, S. K. PI3K/Akt/mTOR and Ras/Raf/MEK/ERK signaling pathways inhibitors as anticancer agents: Structural and pharmacological perspectives. Eur. J. Med. Chem. 109, 314-341 (2016).

35. Philpott, C., Tovell, H., Frayling, I. M., Cooper, D. N. \& Upadhyaya, M. The NF1 somatic mutational landscape in sporadic human cancers. Hum. Genomics 11, 13 (2017).

36. Kaffes, I. et al. Human Mesenchymal glioblastomas are characterized by an increased immune cell presence compared to Proneural and Classical tumors. OncoImmunology 8, e1655360 (2019)

37. Behnan, J., Finocchiaro, G. \& Hanna, G. The landscape of the mesenchymal signature in brain tumours. Brain 142, 847-866 (2019).

38. Liu, K.-W., Hu, B. \& Cheng, S.-Y. Platelet-derived growth factor receptor alpha in glioma: a bad seed. Chin. J. Cancer 30, 590 (2011).

39. Calvert, A. E. et al. Cancer-associated IDH1 promotes growth and resistance to targeted therapies in the absence of mutation. Cell Rep. 19, 1858-1873 (2017).

40. Xu, W. et al. Oncometabolite 2-hydroxyglutarate is a competitive inhibitor of $\alpha$ ketoglutarate-dependent dioxygenases. Cancer Cell 19, 17-30 (2011). 
41. Jenuwein, T. \& Allis, C. D. Translating the histone code. Science 293, 1074-1080 (2001).

42. Chang, S., Yim, S. \& Park, H. The cancer driver genes IDH1/2, JARID1C/ KDM5C , and UTX/ KDM6A: crosstalk between histone demethylation and hypoxic reprogramming in cancer metabolism. Exp. Mol. Med. 51, 1-17 (2019).

43. Dang, L. et al. Cancer-associated IDH1 mutations produce 2-hydroxyglutarate. Nature 462, 739-744 (2009).

44. Malta, T. M. et al. Glioma CpG island methylator phenotype (G-CIMP): biological and clinical implications. Neuro-Oncol. 20, 608-620 (2018).

45. Laug, D., Glasgow, S. M. \& Deneen, B. A glial blueprint for gliomagenesis. Nat. Rev. Neurosci. 19, 393-403 (2018).

46. Shao, F. \& Liu, C. Revisit the candidacy of brain cell types as the cell(s) of origin for human high-grade glioma. Front. Mol. Neurosci. 11, 48 (2018).

47. Bischof, J. et al. Cancer stem cells: The potential role of autophagy, proteolysis, and cathepsins in glioblastoma stem cells: Tumor Biol. (2017) doi:10.1177/1010428317692227.

48. Dawood, S., Austin, L. \& Cristofanilli, M. Cancer stem cells: implications for cancer therapy. Oncol. Williston Park N 28, 1101-1107, 1110 (2014).

49. Hanahan, D. \& Weinberg, R. A. The hallmarks of cancer. Cell 100, 57-70 (2000). 
50. Hanahan, D. \& Weinberg, R. A. hallmarks of cancer: The next generation. Cell 144, 646-674 (2011).

51. Lottaz, C. et al. Transcriptional profiles of CD133+ and CD133-glioblastoma-derived cancer stem cell lines suggest different cells of origin. Cancer Res. 70, 2030-2040 (2010).

52. Batlle, E. \& Clevers, H. Cancer stem cells revisited. Nat. Med. 23, 1124-1134 (2017).

53. Liu, C. et al. Mosaic analysis with double markers reveals tumor cell of origin in glioma. Cell 146, 209-221 (2011).

54. García-Marqués, J., Núñez-Llaves, R. \& López-Mascaraque, L. NG2-glia from pallial progenitors produce the largest clonal clusters of the brain: Time frame of clonal generation in cortex and olfactory Bulb. J. Neurosci. 34, 2305-2313 (2014).

55. Motomura, K. et al. Immunohistochemical analysis-based proteomic subclassification of newly diagnosed glioblastomas. Cancer Sci. 103, 1871-1879 (2012).

56. Ando, T., Kato, R. \& Honda, H. Identification of an early cell fate regulator by detecting dynamics in transcriptional heterogeneity and co-regulation during astrocyte differentiation. Npj Syst. Biol. Appl. 5, 1-9 (2019).

57. Chow, L. M. L. et al. Cooperativity within and among Pten, p53, and Rb pathways induces high-grade astrocytoma in adult brain. Cancer Cell 19, 305-316 (2011). 
58. Vitucci, M. et al. Genomic profiles of low-grade murine gliomas evolve during progression to glioblastoma. Neuro-Oncol. 19, 1237-1247 (2017).

59. Friedmann-Morvinski, D. et al. Dedifferentiation of neurons and astrocytes by oncogenes can induce gliomas in mice. Science 338, 1080-1084 (2012).

60. Patel, A. P. et al. Single-cell RNA-seq highlights intratumoral heterogeneity in primary glioblastoma. Science 344, 1396-1401 (2014).

61. Wang, Q. et al. Tumor evolution of glioma-intrinsic gene expression subtypes associates with immunological changes in the microenvironment. Cancer Cell 32, 4256.e6 (2017).

62. Lee, J. H. et al. Human glioblastoma arises from subventricular zone cells with lowlevel driver mutations. Nature 560, 243-247 (2018).

63. Lenting, K., Verhaak, R., ter Laan, M., Wesseling, P. \& Leenders, W. Glioma: experimental models and reality. Acta Neuropathol. (Berl.) 133, 263-282 (2017).

64. da Hora, C. C., Schweiger, M. W., Wurdinger, T. \& Tannous, B. A. Patient-derived glioma models: From patients to dish to animals. Cells 8, 1177 (2019).

65. Walentynowicz, K. A. et al. In search for reliable markers of glioma-induced polarization of microglia. Front. Immunol. 9, 1329 (2018).

66. Robertson, F. L., Marqués-Torrejón, M.-A., Morrison, G. M. \& Pollard, S. M. Experimental models and tools to tackle glioblastoma. Dis. Model. Mech. 12, (2019). 
67. Gengenbacher, N., Singhal, M. \& Augustin, H. G. Preclinical mouse solid tumour models: status quo, challenges and perspectives. Nat. Rev. Cancer 17, 751-765 (2017).

68. Sharifzad, F. et al. Neuropathological and genomic characterization of glioblastomainduced rat model: How similar is it to humans for targeted therapy? J. Cell. Physiol. (2019) doi:10.1002/jcp.28813.

69. Welker, A. M. et al. Standardized orthotopic xenografts in zebrafish reveal glioma cellline-specific characteristics and tumor cell heterogeneity. Dis. Model. Mech. 9, 199-210 (2016).

70. Agnihotri, S. et al. Glioblastoma, a brief review of history, molecular genetics, animal models and novel therapeutic strategies. Arch. Immunol. Ther. Exp. (Wars\%.) 61, 25-41 (2013).

71. Kim, M. et al. Efficacy of the MDM2 inhibitor SAR405838 in glioblastoma is limited by poor distribution across the blood-brain barrier. Mol. Cancer Ther. 17, 1893-1901 (2018).

72. Xie, Y. et al. The human glioblastoma cell culture resource: Validated cell models representing all molecular subtypes. EBioMedicine 2, 1351-1363 (2015).

73. Translational Neuro-Oncology: Jann N. Sarkaria - Publications. Mayo Clinic https://www.mayo.edu/research/labs/translational-neuro-oncology/mayo-clinicbrain-tumor-patient-derived-xenograft-national-resource/publications. 
74. Ulasov, I. V. et al. TMZ regulates GBM stemness via MMP14-DLL4-Notch3 pathway. Int. J. Cancer 146, 2218-2228 (2020).

75. Stackhouse, C. T. et al. A novel assay for profiling GBM cancer model heterogeneity and drug screening. Cells 8, 702 (2019).

76. Malaney, P., Nicosia, S. V. \& Davé, V. One mouse, one patient paradigm: New avatars of personalized cancer therapy. Cancer Lett. 344, 1-12 (2014).

77. Venkatesan, N., Argus, M. F. \& Arcos, J. C. Mechanism of 3-Methylcholanthreneinduced inhibition of dimethylnitrosamine demethylase in rat liver. Cancer Res. 30, 2556-2562 (1970).

78. Genoud, V. et al. Responsiveness to anti-PD-1 and anti-CTLA-4 immune checkpoint blockade in SB28 and GL261 mouse glioma models. OncoImmunology 7, e1501137 (2018).

79. Ruan, H. et al. Comprehensive characterization of circular RNAs in $\sim 1000$ human cancer cell lines. Genome Med. 11, 55 (2019).

80. Liu, C. J. et al. Treatment of an aggressive orthotopic murine glioblastoma model with combination checkpoint blockade and a multivalent neoantigen vaccine. Neuro-Oncol. 22, 1276-1288 (2020). 
81. Maes, W. \& Van Gool, S. W. Experimental immunotherapy for malignant glioma: lessons from two decades of research in the GL261 model. Cancer Immunol. Immunother. 60, 153-160 (2011).

82. Lee, J. et al. Tumor stem cells derived from glioblastomas cultured in bFGF and EGF more closely mirror the phenotype and genotype of primary tumors than do serumcultured cell lines. Cancer Cell 9, 391-403 (2006).

83. Allen, M., Bjerke, M., Edlund, H., Nelander, S. \& Westermark, B. Origin of the U87MG glioma cell line: Good news and bad news. Sci. Transl. Med. 8, 354re3-354re3 (2016).

84. Gargiulo, G. Next-Generation in vivo Modeling of Human Cancers. Front. Oncol. 8, 429 (2018).

85. Friedel, R. H., Wurst, W., Wefers, B. \& Kühn, R. Generating conditional knockout mice. in Transgenic Mouse Methods and Protocols (eds. Hofker, M. H. \& van Deursen, J.) 205-231 (Humana Press, 2011).

86. Bardella, C. et al. Expression of Idh1R132H in the murine subventricular zone stem cell niche recapitulates features of early gliomagenesis. Cancer Cell 30, 578-594 (2016).

87. Torres-Ruiz, R. \& Rodriguez-Perales, S. CRISPR-Cas9 technology: applications and human disease modelling. Brief. Funct. Genomics 16, 4-12 (2017). 
88. Zhang, J.-H., Adikaram, P., Pandey, M., Genis, A. \& Simonds, W. F. Optimization of genome editing through CRISPR-Cas9 engineering. Bioengineered 7, 166-174 (2016).

89. Hough, S. H. et al. Guide Picker is a comprehensive design tool for visualizing and selecting guides for CRISPR experiments. BMC Bioinformatics 18, 167 (2017).

90. Rodríguez-Rodríguez, D. R., Ramírez-Solís, R., Garza-Elizondo, M. A., De Lourdes Garza-Rodríguez, M. \& Barrera-Saldaña, H. A. Genome editing: A perspective on the application of CRISPR/Cas9 to study human diseases (Review). Int. J. Mol. Med. 43, 1559-1574 (2019).

91. Nambiar, T. S. et al. Stimulation of CRISPR-mediated homology-directed repair by an engineered RAD18 variant. Nat. Commun. 10, 3395 (2019).

92. Huijbers, I. J. Generating genetically modified mice: A decision guide. in Site-Specific Recombinases: Methods and Protocols (ed. Eroshenko, N.) 1-19 (Springer, 2017). doi:10.1007/978-1-4939-7169-5_1.

93. Zuo, E. et al. One-step generation of complete gene knockout mice and monkeys by CRISPR/Cas9-mediated gene editing with multiple sgRNAs. Cell Res. 27, 933-945 (2017).

94. Luther, D. C., Lee, Y. W., Nagaraj, H., Scaletti, F. \& Rotello, V. M. Delivery approaches for CRISPR/Cas9 therapeutics in vivo: advances and challenges. Expert Opin. Drug Deliv. 15, 905-913 (2018). 
95. Chow, R. D. et al. AAV-mediated direct in vivo CRISPR screen identifies functional suppressors in glioblastoma. Nat. Neurosci. 20, 1329-1341 (2017).

96. Xue, W. et al. CRISPR-mediated direct mutation of cancer genes in the mouse liver. Nature 514, 380-384 (2014).

97. Zuckermann, M. et al. Somatic CRISPR/Cas9-mediated tumour suppressor disruption enables versatile brain tumour modelling. Nat. Commun. 6, 7391 (2015).

98. Okabe, M., Ikawa, M., Kominami, K., Nakanishi, T. \& Nishimune, Y. 'Green mice’ as a source of ubiquitous green cells. FEBS Lett. 407, 313-319 (1997).

99. Schneider, R. Intercellular mitochondrial transfer in neural cells a thesis submitted to the Victoria University of Wellington in fulfilment of the requirements for the degree of Doctor of Philosophy in Cell and Molecular Biosciences. (Victoria University of Wellington, 2017).

100. Zhao, S. et al. Glioma-derived mutations in IDH1 dominantly i0nhibit IDH1 catalytic activity and induce HIF-1 $\alpha$. Science 324, 261-265 (2009).

101. Xue, W. et al. CRISPR-mediated direct mutation of cancer genes in the mouse liver. Nature 514, 380-384 (2014).

102. Gao, X. J., Chong, L. S., Kim, M. S. \& Elowitz, M. B. Programmable protein circuits in living cells. Science 361, 1252-1258 (2018). 
103. Rodriguez-Viciana, P. et al. Role of phosphoinositide 3-OH kinase in cell transformation and control of the actin cytoskeleton by Ras. Cell 89, 457-467 (1997).

104. Zhao, S. et al. Glioma-derived mutations in IDH1 dominantly inhibit IDH1 catalytic activity and induce HIF-1 $\alpha$. Science 324, 261-265 (2009).

105. Lisakowski, V. B., Füchtbauer, A. C. \& Füchtbauer, E.-M. Optimized co-transfection of murine embryonic stem cells. Transgenic Res. 27, 131-133 (2018).

106. Levine, A. J. p53, the cellular gatekeeper for growth and division. Cell 88, 323-331 (1997).

107. Chao, C. C.-K. Mechanisms of p53 degradation. Clin. Chim. Acta 438, 139-147 (2015).

108. Williams, A. B. \& Schumacher, B. p53 in the DNA-damage-repair process. Cold Spring Harb. Perspect. Med. 6, (2016).

109. Sánchez-Rivera, F. J. \& Jacks, T. Applications of the CRISPR-Cas9 system in cancer biology. Nat. Rev. Cancer 15, 387-395 (2015).

110. Foster, S. D., Glover, S. R., Turner, A. N., Chatti, K. \& Challa, A. K. A mixing heteroduplex mobility assay (mHMA) to genotype homozygous mutants with small indels generated by CRISPR-Cas9 nucleases. MethodsX 6, 1-5 (2019).

111. Walton, J. B. et al. CRISPR/Cas9-derived models of ovarian high grade serous carcinoma targeting Brca1, Pten and Nf1, and correlation with platinum sensitivity. Sci. Rep. 7, 16827 (2017). 
112. Kosicki, M., Tomberg, K. \& Bradley, A. Repair of double-strand breaks induced by CRISPR-Cas9 leads to large deletions and complex rearrangements. Nat. Biotechnol. 36, 765-771 (2018).

113. Zhang, X.-H., Tee, L. Y., Wang, X.-G., Huang, Q.-S. \& Yang, S.-H. Off-target effects in CRISPR/Cas9-mediated genome engineering. Mol. Ther. Nucleic Acids 4, e264 (2015).

114. Raj, N. \& Attardi, L. D. The transactivation domains of the p53 protein. Cold Spring Harb. Perspect. Med. 7, (2017).

115. Supek, F., Lehner, B. \& Lindeboom, R. G. H. To NMD or not to NMD: Nonsensemediated mRNA decay in cancer and other genetic diseases. Trends Genet. TIG (2020)

116. Aznarez, I. et al. Mechanism of nonsense-mediated mRNA decay stimulation by splicing factor SRSF1. Cell Rep. 23, 2186-2198 (2018).

117. Brogna, S. \& Wen, J. Nonsense-mediated mRNA decay (NMD) mechanisms. Nat. Struct. Mol. Biol. 16, 107-113 (2009).

118. Chang, Y.-F., Imam, J. S. \& Wilkinson, M. F. The nonsense-mediated decay RNA surveillance pathway. Annu. Rev. Biochem. 76, 51-74 (2007).

119. Berridge, M. V., Herst, P. M. \& Tan, A. S. Tetrazolium dyes as tools in cell biology: new insights into their cellular reduction. Biotechnol. Annu. Rev. 11, 127-152 (2005). 
120. Benitez, J. A. et al. PTEN regulates glioblastoma oncogenesis through chromatinassociated complexes of DAXX and histone H3.3. Nat. Commun. 8, 15223 (2017).

121. Koul, D. PTEN Signaling pathways in glioblastoma. Cancer Biol. Ther. 7, 1321-1325 (2008).

122. Malaney, P., Uversky, V. N. \& Davé, V. PTEN proteoforms in biology and disease. Cell. Mol. Life Sci. 74, 2783-2794 (2017).

123. Yan, Y. \& Huang, H. Interplay among PI3K/AKT, PTEN/FOXO and AR signaling in prostate cancer. Prostate Cancer: Cellular and Genetic Mechanisms of Disease Development and Progression (eds. Dehm, S. M. \& Tindall, D. J.) 319-331 (Springer International Publishing, 2019). doi:10.1007/978-3-030-32656-2_14.

124. Useful Numbers for Cell Culture - $\quad$ NZ. //www.thermofisher.com/au/en/home/references/gibco-cell-culture-basics/cellculture-protocols/cell-culture-useful-numbers.html.

125. Carón, R. W. et al. Activated forms of H-RAS and K-RAS differentially regulate membrane association of PI3K, PDK-1, and AKT and the effect of therapeutic kinase inhibitors on cell survival. Mol. Cancer Ther. 4, 257-270 (2005).

126. Chen, S. et al. The function of RAS mutation in cancer and advances in its drug research. Curr. Pharm. Des. 25, 1105-1114 (2019). 
127. Siljamäki, E. et al. H-Ras activation and fibroblast-induced TGF- $\beta$ signaling promote laminin-332 accumulation and invasion in cutaneous squamous cell carcinoma. Matrix Biol. J. Int. Soc. Matrix Biol. 87, 26-47 (2020).

128. Wang, L. et al. K-ras mutation promotes ionizing radiation-induced invasion and migration of lung cancer in part via the Cathepsin L/CUX1 pathway. Exp. Cell Res. 362, 424-435 (2018).

129. Hunn, M. K. et al. Vaccination with irradiated tumor cells pulsed with an adjuvant that stimulates NKT cells is an effective treatment for glioma. Clin. Cancer Res. Off. J. Am. Assoc. Cancer Res. 18, 6446-6459 (2012).

130. Ohgaki, H. \& Kleihues, P. The definition of primary and secondary glioblastoma. Clin. Cancer Res. 19, 764-772 (2013).

131. Zhang, Y.-X., Pan, W.-Y. \& Chen, J. p53 and its isoforms in DNA double-stranded break repair. J. Zhejiang Univ. Sci. B 20, 457-466 (2019).

132. Bunz, F. et al. Requirement for p53 and p21 to sustain G2 arrest after DNA damage. Science 282, 1497-1501 (1998).

133. Warburg, O., Wind, F. \& Negelein, E. The metabolism of tumours in the body. J. Gen. Physiol. 8, 519-530 (1927). 
134. Vaupel, P., Schmidberger, H. \& Mayer, A. The Warburg effect: essential part of metabolic reprogramming and central contributor to cancer progression. Int. J. Radiat. Biol. 95, 912-919 (2019).

135. Liu, J., Zhang, C., Hu, W. \& Feng, Z. Tumor suppressor p53 and metabolism. J. Mol. Cell Biol. 11, 284-292 (2019).

136. Eriksson, M. et al. Effect of mutant p53 proteins on glycolysis and mitochondrial metabolism. Mol. Cell. Biol. 37, (2017).

137. Tchelebi, L., Ashamalla, H. \& Graves, P. R. Mutant p53 and the response to chemotherapy and radiation. Subcell. Biochem. 85, 133-159 (2014).

138. Taylor, W. R. \& Stark, G. R. Regulation of the G2/M transition by p53. Oncogene 20, 1803-1815 (2001).

139. Moure, C. J. et al. CRISPR editing of mutant IDH1 R132H induces a CpG methylation-low state in patient-derived glioma models of G-CIMP. Mol. Cancer Res. 17, 2042-2050 (2019).

140. Waitkus, M. S., Diplas, B. H. \& Yan, H. Biological role and therapeutic potential of IDH mutations in cancer. Cancer Cell 34, 186-195 (2018).

141. Turcan, S. et al. Mutant-IDH1-dependent chromatin state reprogramming, reversibility, and persistence. Nat. Genet. 50, 62-72 (2018). 
142. Fedele, M., Cerchia, L., Pegoraro, S., Sgarra, R. \& Manfioletti, G. Proneuralmesenchymal transition: Phenotypic plasticity to acquire multitherapy resistance in glioblastoma. Int. J. Mol. Sci. 20, (2019).

143. Zhang, Z. et al. Transfer of microRNA via macrophage-derived extracellular vesicles promotes proneural-to-mesenchymal transition in glioma stem cells. Cancer Immunol. Res. 8, 966-981 (2020).

144. Fabre, M.-S. et al. The oncogene BCL6 is up-regulated in glioblastoma in response to DNA damage, and drives survival after therapy. PLOS ONE 15, e0231470 (2020).

145. Xu, L. et al. BCL6 promotes glioma and serves as a therapeutic target. Proc. Natl. Acad. Sci. U. S. A. 114, 3981-3986 (2017). 


\section{Appendix}

\section{Proliferation Assay}

\begin{tabular}{|c|c|c|c|c|c|c|c|c|c|}
\hline \multirow{2}{*}{\multicolumn{2}{|c|}{$\begin{array}{c}\text { Table format: } \\
\text { XY }\end{array}$}} & $\mathrm{X}$ & \multicolumn{3}{|c|}{ Group A } & \multicolumn{3}{|c|}{ Group B } & \multirow[b]{3}{*}{ C:Y1 } \\
\hline & & Elapsed time & & Astrocytes & & & $57 \mathrm{C} 3$ Pten & & \\
\hline 4 & $\mathbf{x}$ & Time hh:mm:ss & A:Y1 & A:Y2 & A:Y3 & B:Y1 & B:Y2 & $\mathrm{B}: \mathrm{Y} 3$ & \\
\hline 1 & Title & $0: 00: 00$ & 3.117285 & 4.659047 & 3.673977 & 2.283901 & 4.760578 & 2.680002 & \\
\hline 2 & Title & 4:00:00 & 3.572058 & 8.632208 & 4.192670 & 2.805164 & 8.535337 & 3.558691 & \\
\hline 3 & Title & 8:00:00 & 4.399499 & 8.047804 & 5.323504 & 3.493788 & 8.662082 & 4.425112 & \\
\hline 4 & Title & $12: 00: 00$ & 5.135046 & 8.313297 & 6.361356 & 4.592452 & 10.186480 & 5.526426 & \\
\hline 5 & Title & $16: 00: 00$ & 6.638307 & 10.146890 & 7.544323 & 5.795300 & 12.289720 & 6.993977 & \\
\hline 6 & Title & $20: 00: 00$ & 14.598530 & 12.959560 & 8.463396 & 12.366000 & 15.536560 & 8.124171 & \\
\hline 7 & Title & $24: 00: 00$ & 9.994917 & 14.393420 & 10.095540 & 10.212390 & 18.886680 & 10.333880 & \\
\hline 8 & Title & 28:00:00 & 11.006140 & 19.643200 & 12.355250 & 12.008980 & 25.375270 & 12.478310 & \\
\hline 9 & Title & $32: 00: 00$ & 20.694010 & 20.708500 & 13.977440 & 22.034970 & 27.007030 & 15.252650 & \\
\hline 10 & Title & $36: 00: 00$ & 17.135220 & 24.284300 & 16.627050 & 20.388300 & 32.386380 & 18.715190 & \\
\hline 11 & Title & $40: 00: 00$ & 18.514800 & 29.982700 & 18.176980 & 22.460470 & 39.167060 & 22.126630 & \\
\hline 12 & Title & $44: 00: 00$ & 19.083560 & 34.814030 & 21.437080 & 28.155330 & 45.039270 & 26.768440 & \\
\hline 13 & Title & $48: 00: 00$ & 21.988500 & 38.427410 & 36.744820 & 31.710410 & 49.947400 & 32.264720 & \\
\hline 14 & Title & $52: 00: 00$ & 39.142680 & 44.660100 & 31.398510 & 48.843830 & 57.799910 & 38.892810 & \\
\hline 15 & Title & $56: 00: 00$ & 38.935330 & 50.548310 & 34.168840 & 49.481540 & 64.922290 & 45.527070 & \\
\hline 16 & Title & $60: 00: 00$ & 39.256520 & 56.988960 & 39.295780 & 52.869050 & 67.995670 & 52.322660 & \\
\hline 17 & Title & $64: 00: 00$ & 41.338910 & 64.169340 & 41.464140 & 58.384210 & 75.752840 & 57.093610 & \\
\hline 18 & Title & $68: 00: 00$ & 65.269530 & 70.644660 & 45.726130 & 76.417950 & 79.866110 & 73.098610 & \\
\hline 19 & Title & $72: 00: 00$ & 64.777570 & 70.829010 & 53.057710 & 78.322660 & 82.206390 & 73.056570 & \\
\hline 20 & Title & $76: 00: 00$ & 64.129070 & 74.703000 & 55.321450 & 78.539830 & 87.807500 & 77.802030 & \\
\hline 21 & Title & $80: 00: 00$ & 65.992530 & 74.672040 & 66.429430 & 80.802290 & 89.217120 & 81.978320 & \\
\hline 22 & Title & $84: 00: 00$ & 68.600620 & 80.884790 & 64.786680 & 83.823980 & 91.977690 & 85.660760 & \\
\hline 23 & Title & $88: 00: 00$ & 84.169570 & 82.508070 & 81.694680 & 92.451260 & 93.551900 & 95.783100 & \\
\hline 24 & Title & Elapsed time & & & & & & & \\
\hline
\end{tabular}

Biological triplicate data from growth curve assay for astrocytes and C57C3 Pten cells 


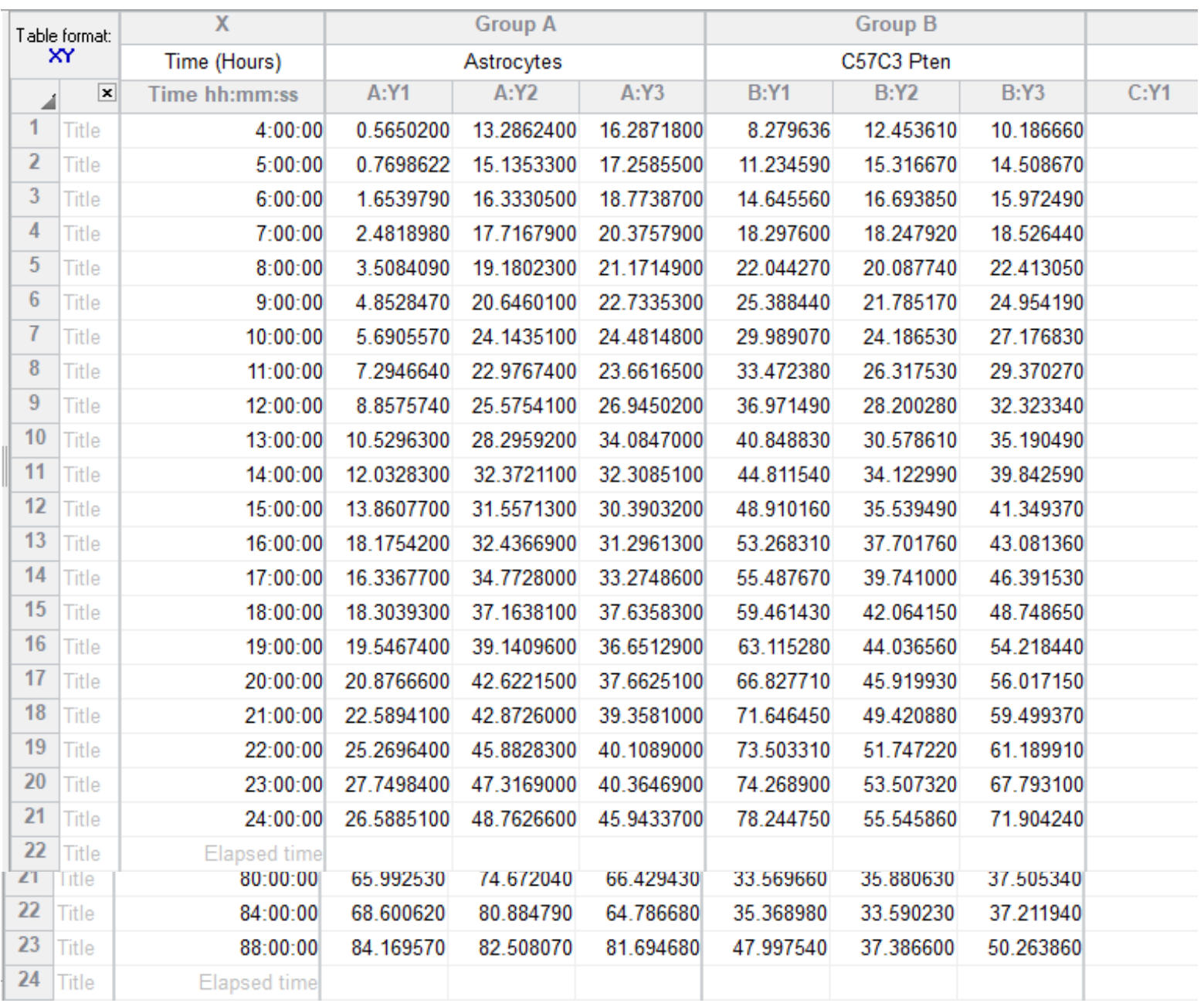

Biological triplicate data from growth curve assay for astrocytes and C57F3 P53 cells 


\section{Wound Healing Assay}

\begin{tabular}{|c|c|c|c|c|c|c|c|c|c|}
\hline \multirow{2}{*}{\multicolumn{2}{|c|}{$\begin{array}{l}\text { Table format: } \\
\text { XY }\end{array}$}} & \multirow{3}{*}{$\begin{array}{c}\text { X } \\
\text { Time (Hours) } \\
\text { Time hh:mm:ss }\end{array}$} & \multicolumn{3}{|c|}{ Group A } & \multicolumn{3}{|c|}{ Group B } & \multirow[b]{3}{*}{$\mathrm{C}: \mathrm{Y1}$} \\
\hline & & & \multicolumn{3}{|c|}{ C57C3 Pten } & \multicolumn{3}{|c|}{ Pten + RAS } & \\
\hline$\Delta$ & $x$ & & A:Y1 & A:Y2 & A:Y3 & B:Y1 & B:Y2 & B:Y3 & \\
\hline 1 & Title & $4: 00: 00$ & 8.279636 & 12.453610 & 10.186660 & 10.233040 & 15.129450 & 10.176150 & \\
\hline 2 & Title & $5: 00: 00$ & 11.234590 & 15.316670 & 14.508670 & 13.806550 & 18.810420 & 10.726020 & \\
\hline 3 & Title & $6: 00: 00$ & 14.645560 & 16.693850 & 15.972490 & 17.376960 & 20.311400 & 13.453810 & \\
\hline 4 & Title & $7: 00: 00$ & 18.297600 & 18.247920 & 18.526440 & 21.388570 & 22.876610 & 14.964050 & \\
\hline 5 & Title & 8:00:00 & 22.044270 & 20.087740 & 22.413050 & 25.771810 & 25.470050 & 18.183080 & \\
\hline 6 & Title & 9:00:00 & 25.388440 & 21.785170 & 24.954190 & 30.098120 & 27.675000 & 23.573780 & \\
\hline 7 & Title & $10: 00: 00$ & 29.989070 & 24.186530 & 27.176830 & 35.256290 & 30.165200 & 23.757560 & \\
\hline 8 & Title & $11: 00: 00$ & 33.472380 & 26.317530 & 29.370270 & 38.257640 & 32.979210 & 28.227480 & \\
\hline 9 & Title & $12: 00: 00$ & 36.971490 & 28.200280 & 32.323340 & 42.771560 & 36.187110 & 29.174260 & \\
\hline 10 & Title & $13: 00: 00$ & 40.848830 & 30.578610 & 35.190490 & 46.906330 & 39.338330 & 32.559400 & \\
\hline 11 & Title & $14: 00: 00$ & 44.811540 & 34.122990 & 39.842590 & 51.530390 & 42.292950 & 37.225770 & \\
\hline 12 & Title & $15: 00: 00$ & 48.910160 & 35.539490 & 41.349370 & 55.251390 & 44.687240 & 38.363520 & \\
\hline 13 & Title & $16: 00: 00$ & 53.268310 & 37.701760 & 43.081360 & 61.411330 & 47.918600 & 41.258130 & \\
\hline 14 & Title & $17: 00: 00$ & 55.487670 & 39.741000 & 46.391530 & 65.403620 & 50.250480 & 44.810430 & \\
\hline 15 & Title & $18: 00: 00$ & 59.461430 & 42.064150 & 48.748650 & 67.930350 & 53.308940 & 49.658930 & \\
\hline 16 & Title & $19: 00: 00$ & 63.115280 & 44.036560 & 54.218440 & 71.849270 & 55.440910 & 51.443840 & \\
\hline 17 & Title & $20: 00: 00$ & 66.827710 & 45.919930 & 56.017150 & 75.119660 & 57.143970 & 53.632200 & \\
\hline 18 & Title & $21: 00: 00$ & 71.646450 & 49.420880 & 59.499370 & 80.760640 & 61.812410 & 57.224780 & \\
\hline 19 & Title & $22: 00: 00$ & 73.503310 & 51.747220 & 61.189910 & 82.396140 & 72.548510 & 63.431540 & \\
\hline 20 & Title & $23: 00: 00$ & 74.268900 & 53.507320 & 67.793100 & 84.552580 & 77.303440 & 68.834130 & \\
\hline 21 & Title & $24: 00: 00$ & 78.244750 & 55.545860 & 71.904240 & 88.193860 & 79.113410 & 77.992940 & \\
\hline 22 & Title & Elapsed time & & & & & & & \\
\hline
\end{tabular}

Biological triplicate data from wound healing assay for C57C3 Pten and Pten + RAS cells 


\section{MTS Assay}

\begin{tabular}{|c|c|c|c|c|c|c|c|c|c|}
\hline \multirow{2}{*}{\multicolumn{2}{|c|}{$\begin{array}{l}\text { Table format: } \\
\text { XY }\end{array}$}} & $\mathrm{X}$ & \multicolumn{3}{|c|}{ Group A } & \multicolumn{3}{|c|}{ Group B } & \multirow[b]{3}{*}{ C:Y1 } \\
\hline & & Time (Hours) & \multicolumn{3}{|c|}{ Astrocytes } & \multicolumn{3}{|c|}{ C57C3 Pten } & \\
\hline$\Delta$ & $\mathbf{x}$ & Time hh:mm:ss & A:Y1 & A:Y2 & A:Y3 & B:Y1 & B:Y2 & B:Y3 & \\
\hline 2 & Title & $48: 00: 00$ & 0.67944444 & 0.78733333 & 0.40355600 & 0.720888889 & 1.068222220 & 0.797444000 & \\
\hline 3 & Title & $72: 00: 00$ & 0.66255556 & 0.80311111 & 0.78988900 & 1.245111111 & 1.275888890 & 1.044444000 & \\
\hline 5 & Title & Elapsed time & & & & & & & \\
\hline
\end{tabular}

Biological triplicate data from MTS assay for astrocytes and C57C3 Pten cells

\begin{tabular}{|c|c|c|c|c|c|c|c|c|c|c|c|c|}
\hline \multirow{2}{*}{\multicolumn{2}{|c|}{$\begin{array}{l}\text { Table format: } \\
\text { XY }\end{array}$}} & $\mathrm{x}$ & \multicolumn{3}{|c|}{ Group A } & \multicolumn{3}{|c|}{ Group B } & \multicolumn{3}{|c|}{ Group C } & \multirow[b]{3}{*}{$\mathrm{D}: \mathrm{Y1}$} \\
\hline & & Time (Hours) & & C57C3 Pten & & & en + RAS & & & Pten + EGFR & & \\
\hline 2 & $x$ & Time hh:mm:ss & A:Y1 & A:Y2 & A:Y3 & B:Y1 & B:Y2 & B:Y3 & C:Y1 & C:Y2 & C:Y3 & \\
\hline 1 & Title & $24: 00: 00$ & 0.446111111 & 0.559777780 & 0.440222000 & 0.428000 & 0.562444 & 0.398222 & 0.49766667 & 0.44944400 & 0.41644400 & \\
\hline 2 & Title & $48: 00: 00$ & 0.720888889 & 1.0682222220 & 0.797444000 & 0.703889 & 0.972778 & 0.559778 & 0.76933333 & 0.67655600 & 0.71766700 & \\
\hline 3 & Title & $72: 00: 00$ & 1.245111111 & 1.275888890 & 1.044444000 & 1.093111 & 0.949667 & 0.974889 & 1.31533333 & 1.10222200 & 1.13800000 & \\
\hline 4 & Title & $96: 00: 00$ & 1.213888889 & 0.949333330 & 1.136246500 & 0.952889 & 0.870222 & 0.945213 & 1.00500000 & 1.28654200 & 1.29188900 & \\
\hline 5 & Title & Elapsed time & & & & & & & & & & \\
\hline
\end{tabular}

Biological triplicate data from MTS assay for 'classical-like GEM-CLeMs

\begin{tabular}{|c|c|c|c|c|c|c|c|c|c|}
\hline & & \multicolumn{3}{|c|}{ Group A } & \multicolumn{3}{|c|}{ Group B } & \multirow{3}{*}{ C:Y1 } \\
\hline & & & \multicolumn{3}{|c|}{ Astrocytes } & \multicolumn{3}{|c|}{ C57F3 P53 } & \\
\hline $1^{4} x^{x}$ & $\begin{array}{l}\text { Table format: } \\
\text { XY }\end{array}$ & X & A:Y1 & A:Y2 & A:Y3 & B:Y1 & $\mathrm{B}: Y 2$ & B:Y3 & \\
\hline \multirow{2}{*}{$\begin{array}{l}2 \\
3\end{array}$} & Title & $\begin{array}{r}24: 00: 00 \\
48: 00: 00\end{array}$ & 0.67944444 & 0.78733333 & 0.40355600 & 0.4931111 & \multicolumn{2}{|c|}{$0.6403333 \quad 0.3712220$} & \\
\hline & Title & $72: 00: 00$ & 0.66255556 & 0.80311111 & 0.78988900 & 0.4017778 & \multicolumn{2}{|c|}{$0.7306667 \quad 0.5104440$} & \\
\hline 5 & Title & Elapsed time & & & & & \multicolumn{2}{|c|}{ 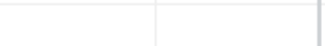 } & \\
\hline
\end{tabular}

Biological triplicate data from MTS assay for astrocytes and C57F3 P53 cells 


\begin{tabular}{|c|c|c|c|c|c|c|c|c|c|}
\hline \multirow{2}{*}{\multicolumn{2}{|c|}{$\begin{array}{c}\text { Table format: } \\
\text { XY }\end{array}$}} & $\mathrm{X}$ & \multicolumn{3}{|c|}{ Group A } & \multicolumn{3}{|c|}{ Group B } & \multirow[b]{3}{*}{ C:Y1 } \\
\hline & & Time (Hours) & \multicolumn{3}{|c|}{ C57F3 P53 } & \multicolumn{3}{|c|}{ C57F3 P53 + IDH1 } & \\
\hline 4 & $\mathbf{x}$ & Time hh:mm:ss & A:Y1 & A:Y2 & A:Y3 & B:Y1 & B:Y2 & B:Y3 & \\
\hline 1 & Title & $24: 00: 00$ & 0.3712222 & 0.5214444 & 0.2353330 & 0.43977778 & 0.11822200 & 0.25477800 & \\
\hline 2 & Title & $48: 00: 00$ & 0.4931111 & 0.6403333 & 0.3712220 & 0.47855556 & 0.26066700 & 0.29711100 & \\
\hline 3 & Title & $72: 00: 00$ & 0.4017778 & 0.7306667 & 0.5104440 & 0.57688889 & 0.33744400 & 0.43155600 & \\
\hline 4 & Title & $96: 00: 00$ & 0.2720000 & 0.4042222 & 0.3845270 & 0.33477778 & 0.35623440 & 0.45644400 & \\
\hline 5 & Title & Elapsed time & & & & & & & \\
\hline
\end{tabular}

Biological triplicate data from MTS assay for 'secondary-like' GEM-CLeMs

\section{Clonogenic Assay}
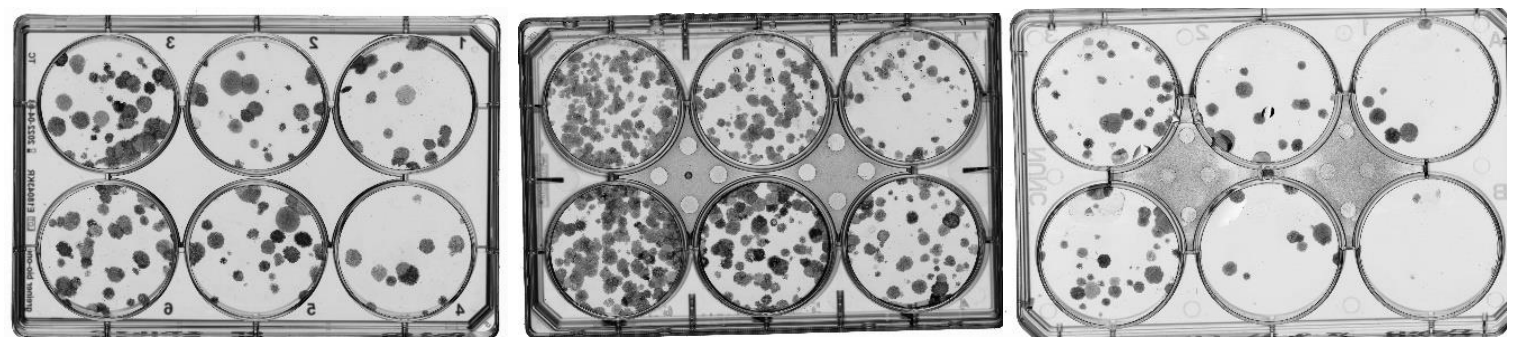

Biological triplicate plates for the astrocytes clonogenic assay

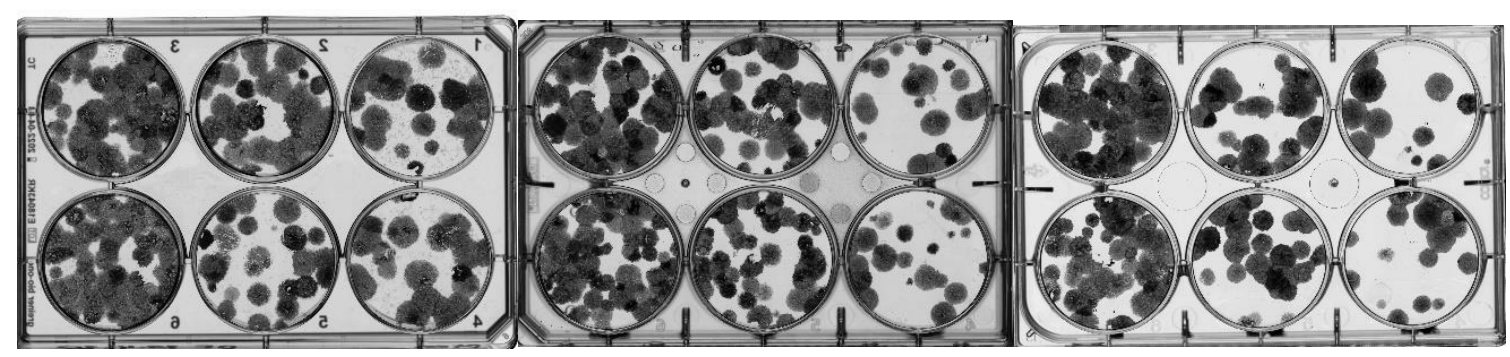

Biological triplicate plates for the C57C3 Pten clonogenic assay 


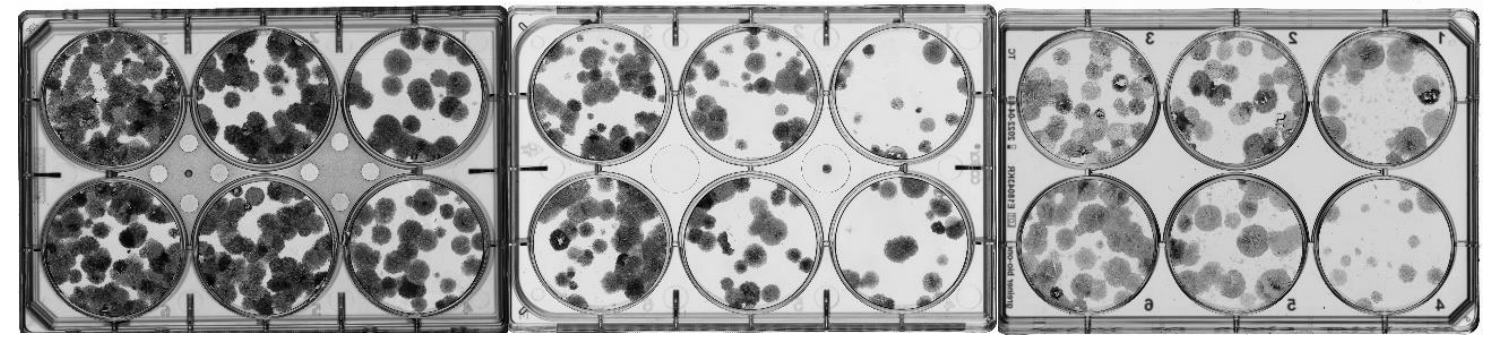

Pten + RAS
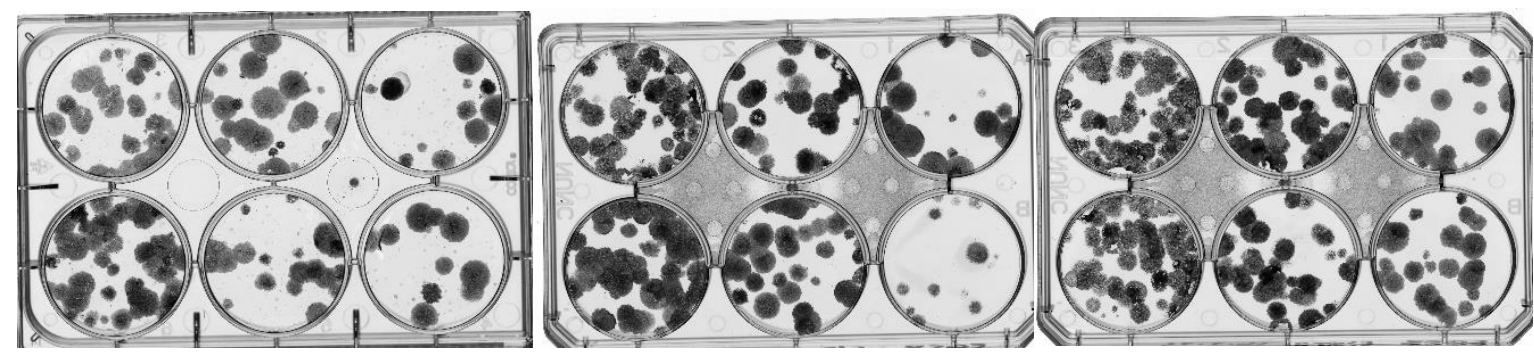

Biological triplicate plates for the Pten + EGFR clonogenic assay 


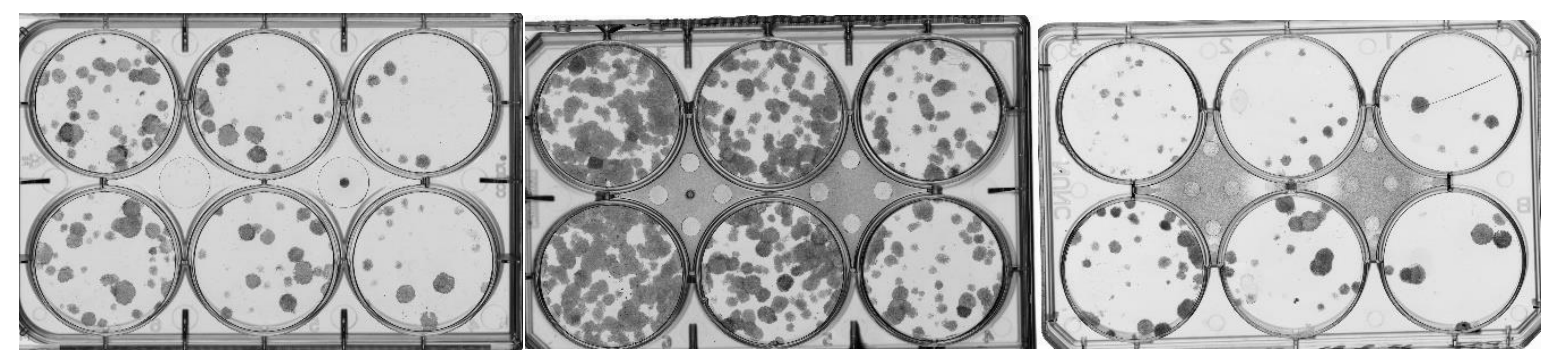

Biological triplicate plates for the C57F3 P53 clonogenic assay

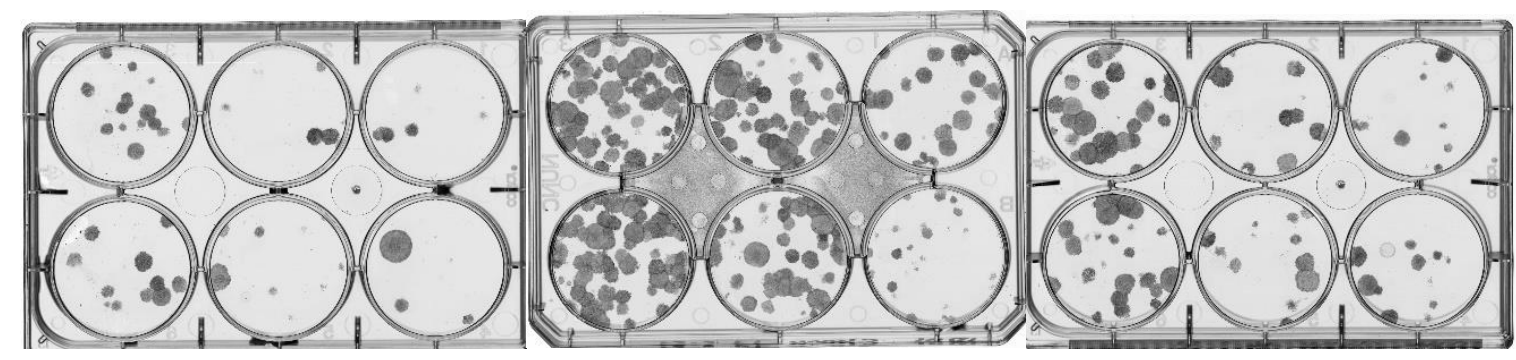

Biological triplicate plates for the P53 + IDH1 clonogenic assay 